Supporting Information

\title{
Programmed Sequential Additions to Halogenated Mucononitriles
}

Adam J. Zahara, Elsa M. Hinds, Andrew L. Nguyen, and Sidney M. Wilkerson-Hill*

Department of Chemistry, The University of North Carolina at Chapel Hill Chapel Hill, NC 27599

\section{Supporting Information}

I. General Information $\quad$ S1

II. Optimization of Chlorination Reaction of Benzothiadiazole $\quad$ S3

III. Preparation and Characterization of $\mathbf{3 a}$ and $\mathbf{3 b} \quad S 4$

IV. Preparation and Characterization of Substrates $\quad$ S6

V. Preparation and Characterization of Amine Addition Products $\quad$ S10

VI. Preparation and Characterization of Malonate Additions Products $\quad$ S24

VII. Preparation and Characterization of Functionalization of $\mathbf{7 a}$ and $\mathbf{7 b}$ Products $\quad$ S31

VIII. Preparation and Characterization of Interrupted Addition to 3b Products S33

IX. Preparation and Characterization of Annulation Reaction Products $\quad$ S35

$\begin{array}{ll}\text { X. Descriptions of Stereochemical Assignments } & \text { S38 }\end{array}$

$\begin{array}{ll}\text { XI. X-ray Data for 3a and 19a } & \text { S44 }\end{array}$

$\begin{array}{ll}\text { XII. References } & \text { S55 }\end{array}$

$\begin{array}{ll}\text { XIII. Copies of NMR Spectra } & \text { S56 }\end{array}$

\section{General Information}

Reaction setup and reagent purification: All reactions were carried out under a nitrogen atmosphere in flame-dried glassware using standard Schlenk techniques unless otherwise stated. Reactions that were extremely moisture and oxygen sensitive reactions were performed within a Vigor glove box (box atmospheric pressure ranged from 3-5 atm, moisture and oxygen ranged from $0.10-0.20 \mathrm{ppm}$ and 0.01 and $0.02 \mathrm{ppm}$ respectively). All reagents were purchased from commercial sources and were used as received unless otherwise noted. $\mathrm{N}$-methyl-1-(naphthalen-2-yl)methanamine $\mathrm{HCl},{ }^{1}$ diethyl 2-cinnamylmalonate, ${ }^{2}$ diethyl 2-(furan-2-ylmethyl)malonate, ${ }^{3}$ and diethyl 2-((1H-indol-3-yl)methyl)malonate ${ }^{4}$ were prepared according to previously reported procedures and spectral data matched those reported. 
Solvents: Toluene, tetrahydrofuran, dichloromethane, diethyl ether, acetonitrile, and triethylamine were purified using a GlassContour solvent purification system purchased from Pure Process Technologies. Other solvents (hexanes and pentanes) were purified by refluxing over activated $3 \AA$ molecular sieves (beads) for 1 hour and stored as such under a balloon filled with Ar. Anhydrous 1,2-dichloroethane and 1,4-dioxane were purchased from Acros and Dri-Solv, respectively, and were used without purification.

Chromatography: Analytical thin layer chromatography (TLC) was performed on SiliaPlate glass backed silica gel plates (250 mm thickness, $60 \AA$, F-254 indicator) purchased from Silicycle and visualized using ultraviolet light (254 and $200 \mathrm{~nm}$ ) and stained with $\mathrm{KMNO}_{4}, p$-anisaldehyde, cerium molybdate, ceric ammonium nitrate, vanillin, or ninhydrin following reported procedures. ${ }^{5}$ Flash column chromatography was performed with SiliaFlash silica gel $60 \AA$ A, 230-400 mesh (40-63 mm) purchased from Silicycle according to the literature procedure.

Melting points: All melting points were measured on powdered solids using a Mel-Temp II capillary melting point device and are uncorrected unless otherwise stated.

Nuclear Magnetic Resonance Characterization: ${ }^{1} \mathrm{H}$ NMR and ${ }^{13} \mathrm{C}$ NMR spectra were recorded on a Bruker AV Neo-400 (equipped with a Prodigy cryoprobe), Bruker AV III NanoBay-400, Bruker AV III500, Bruker AV Neo-600 (equipped with a CryoQNP probe) or Bruker AV III-600 MHz spectrometer (100, 125 , or $150 \mathrm{MHz}$ for $\left.{ }^{13} \mathrm{C} \mathrm{NMR}\right)$. All spectra were recorded in deuterated chloroform $\left(\mathrm{CDCl}_{3}\right)$ with residual chloroform ( $\delta=7.26 \mathrm{ppm}$ for ${ }^{1} \mathrm{H}$ NMR and 77.16 for $\left.{ }^{13} \mathrm{C} \mathrm{NMR}\right)$ as the internal standard and are reported in parts per million (ppm). Abbreviations for the signal couplings are as follows: s, singlet; $d$, doublet; $t$, triplet; q, quartet; quin, quintet; sex, sextet; sept; septet; and m, multiplet. Coupling constants were taken from the spectra directly and are uncorrected. Structural and stereochemical assignments were also made based on analysis of ${ }^{13} \mathrm{C}$ APT, 2-D ${ }^{1} \mathrm{H}-{ }^{1} \mathrm{H}$ COSY and ${ }^{1} \mathrm{H}-{ }^{1} \mathrm{H}$ NOESY, ${ }^{1} \mathrm{H}-{ }^{13} \mathrm{C}$ HSQC and ${ }^{1} \mathrm{H}-{ }^{13} \mathrm{C}$ HMBC experiments. All diastereomeric ratios were determined by ${ }^{1} \mathrm{H}$ NMR analysis of an aliquot from the crude reaction mixture. Yields refer to isolated yields of compounds estimated to be $\geq 95 \%$ pure as determined by ${ }^{1} \mathrm{H}$ NMR analysis unless otherwise noted.

Infrared Spectroscopy Characterization: Infrared (IR) spectra were collected on a Thermo Scientific Nicolet iS 5 FT-IR. Compounds were analyzed as thin films or as solids on the diamond surface. Spectra are reported in frequency of absorption in $\mathrm{cm}^{-1}$. Only selected references are reported. 
Mass spectrometry: High-resolution mass spectra (HRMS) determinations were performed by the mass spectral facility at the University of North Carolina at Chapel Hill. Samples were analyzed with a Q Exactive HF-X (ThermoFisher, Bremen, Germany) mass spectrometer for APCI and ESI, on a ThermoFisher GC Exactive with an Electron Ionization (EI) source using an Orbitrap detector.

\section{General chlorination procedure - General Procedure A}

A flame-dried $4 \mathrm{~mL}$ vial equipped with a magnetic Teflon ${ }^{\circledR}$-coated stir bar was charged with 2,1,3benzothiadiazole (4) $\left(68.0 \mathrm{mg}, 511 \mu \mathrm{mol}, 1.00\right.$ equiv), $\mathrm{H}_{2} \mathrm{SO}_{4}(500 \mu \mathrm{L}, 510 \mu \mathrm{mol}, 1.00$ equiv), chlorinating reagent (1.00 mmol, 2.00 equiv) portionwise over 1-2 minutes. The mixture was stirred opened to air for 12 hours at $45^{\circ} \mathrm{C}$ and the solid dissolved upon heating. The solution was cooled to $0{ }^{\circ} \mathrm{C}$, and quenched with cold aqueous saturated sodium bicarbonate (ca. $12 \mathrm{~mL}$, until bubbling subsided). The mixture was extracted with ethyl acetate $(3 \times 2 \mathrm{~mL})$, dried over $\mathrm{Na}_{2} \mathrm{SO}_{4}$, filtered and concentrated to give off-white/yellow solid, which was analyzed by ${ }^{1} \mathrm{H}$ NMR.

\section{Optimization of Chlorination of Benzothiadiazole}

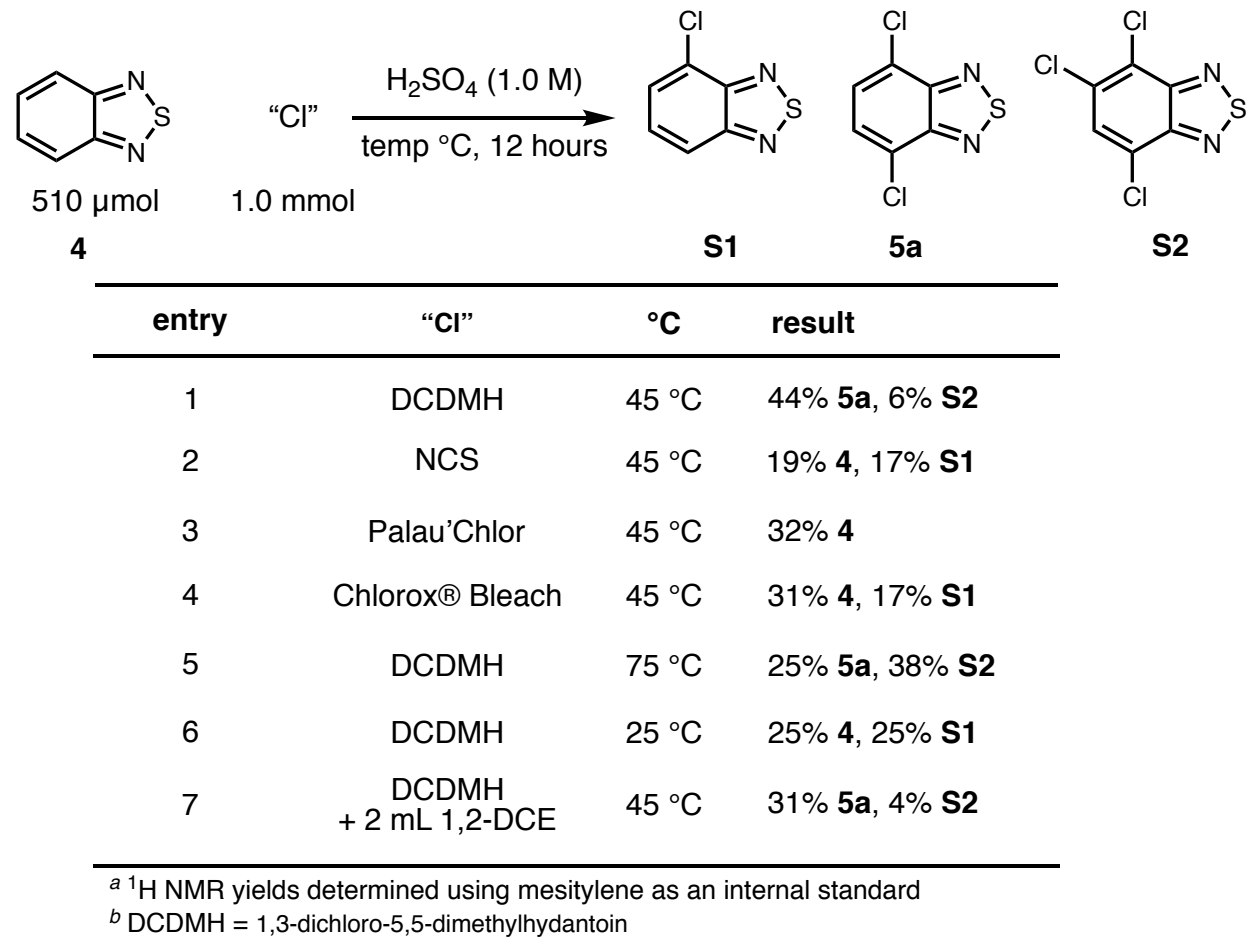

\section{Preparation and Characterization of $3 \mathrm{a}$ and $\mathbf{3 b}$}


<smiles>Clc1ccc(Cl)c2nsnc12</smiles>

4,7-Dichlorobenzo[c][1,2,5]thiadiazole (5a). A flame-dried $500 \mathrm{~mL}$ Erlenmeyer flask equipped with a magnetic Teflon ${ }^{\circledR}$-coated stir bar was cooled then charged with benzothiadiazole (4) (13.6 g, $100 \mathrm{mmol}$, 1.00 equiv), $\mathrm{H}_{2} \mathrm{SO}_{4}(200 \mathrm{~mL}, 500 \mathrm{mM})$, and 1,3-dichloro-5,5,-dimethylhydantoin (39.4 g, $200 \mathrm{mmol}, 2.00$ equiv) portionwise over 10 minutes. The mixture was stirred opened to air for 12 hours at $45{ }^{\circ} \mathrm{C}$ and the solid dissolved upon heating. The solution was cooled to room temperature then poured over roughly 500 $\mathrm{g}$ of crushed ice in a $1 \mathrm{~L}$ Erlenmeyer flask. Upon warming to $23{ }^{\circ} \mathrm{C}$, the solids were collected in a Büchner funnel using course porosity filter paper, and the filter cake was washed with $200 \mathrm{~mL}$ of water. Once dry, the solid residue was recrystallized from hot ethanol to afford the title compound as colorless needles (9.68 g, 47\%, $47.2 \mathrm{mmol})$.

$\mathbf{R}_{\mathbf{f}}(91: 9$ hexanes:ethyl acetate $)=0.79$.

m.p. $178-180{ }^{\circ} \mathrm{C}$ (ethanol).

${ }^{1} \mathbf{H}$ NMR $\left(600 \mathrm{MHz}, \mathrm{CDCl}_{3}\right) \delta 7.58(\mathrm{~s}, 2 \mathrm{H})$.

${ }^{13} \mathbf{C}$ NMR (151 MHz, $\left.\mathrm{CDCl}_{3}\right) \delta 152.5,128.6,125.0$.

IR vmax (film) 3085, 3051, 2951, 2922, 1717, $1510 \mathrm{~cm}^{-1}$.

HRMS (APCI) m/z: [M+H] $]^{+}$Calcd for $\mathrm{C}_{6} \mathrm{H}_{3} \mathrm{Cl}_{2} \mathrm{~N}_{2} \mathrm{~S}$ 204.9394; Found 204.9391.<smiles>N#C/C(Cl)=C\C=C(Cl)Cl</smiles>

(2E,4E)-2,5-Dichlorohexa-2,4-dienedinitrile (3a). A flame-dried $1 \mathrm{~L}$ round bottomed flask equipped with a magnetic Teflon ${ }^{\circledR}$-coated stir bar was cooled under a stream of nitrogen and charged with 4,7dichlorobenzo[c][1,2,5]thiadiazole (5a) $(10.3 \mathrm{~g}, 50.0 \mathrm{mmol}, 1.00$ equiv) and ethanol ( $250 \mathrm{~mL}, 0.20 \mathrm{M})$, and the flask was placed in an ice bath. To the stirred solution was added sodium borohydride (14.2 $\mathrm{g}, 375$ mmol, 7.50 equiv) portionwise over the course of 1 hour, after which the flask was allowed to warm to room temperature. After 18 hours the solvent was removed in vacuo and crude residue was taken up in ethyl acetate $(300 \mathrm{~mL})$ then saturated aqueous sodium bicarbonate solution $(200 \mathrm{~mL})$ was carefully added. The mixture was placed in a separatory funnel and the aqueous layer was separated then extracted with ethyl acetate $(2 \times 100 \mathrm{~mL})$, then the combined organic extracts were washed with brine and dried over 
$\mathrm{Na}_{2} \mathrm{SO}_{4}$ then filtered. The solvent was removed in vacuo and the crude residue was dried under high-vacuum overnight and carried forward without further purification. To an oven-dried $500 \mathrm{~mL}$ round bottomed flask was added lead (IV) tetraacetate (44.3 g, $100 \mathrm{mmol}, 2.00$ equiv), $100 \mathrm{~mL}$ of anhydrous toluene, and a magnetic Teflon ${ }^{\circledR}$-coated stir bar. The flask was heated to $60{ }^{\circ} \mathrm{C}$ in an oil bath with stirring, then equipped with a slow-addition funnel. To the addition funnel was added the crude ortho-phenylenediamine intermediate in $150 \mathrm{~mL}$ of anhydrous toluene, and the solution was added dropwise over the course of 15 minutes. The reaction mixture was heated to $60{ }^{\circ} \mathrm{C}$ for 2 hours, then was cooled to room temperature and filtered over Celite ${ }^{\circledR}$ and rinsed with dichloromethane. To the mixture was added saturated aqueous sodium bicarbonate solution $(200 \mathrm{~mL})$, and the material was extracted with dichloromethane $(2 \times 100 \mathrm{~mL})$ in a separatory funnel, then the combined organic layers were washed with brine, dried over $\mathrm{Na}_{2} \mathrm{SO}_{4}$, and filtered. The solvent was removed in vacuo and the crude residue was recrystallized from hot toluene to afford the title compound as a pale yellow crystalline solid (2.75 g, 32\%, $16.0 \mathrm{mmol})$.

m.p. $161-163^{\circ} \mathrm{C}$.

$\mathbf{R}_{\mathbf{f}}(90: 10$ hexanes:ethyl acetate $)=0.57$.

${ }^{1} \mathbf{H}$ NMR $\left(600 \mathrm{MHz}, \mathrm{CDCl}_{3}\right) \delta 7.27(\mathrm{~s}, 2 \mathrm{H})$.

${ }^{13} \mathbf{C}$ NMR (151 MHz, $\left.\mathrm{CDCl}_{3}\right) \delta 137.7,112.5,111.1$.

IR (ATR) 3039, 2225, $1522 \mathrm{~cm}^{-1}$.

HRMS (APCI) m/z: [M] ${ }^{+}$Calcd for $\mathrm{C}_{6} \mathrm{H}_{2} \mathrm{Cl}_{2} \mathrm{~N}_{2}$ 171.9595; Found 171.9591.<smiles>N#C/C(Br)=C\C=C(Br)Br</smiles>

(2E,4E)-2,5-Dibromohexa-2,4-dienedinitrile (3b). A flame-dried 1 L round bottomed flask equipped with a magnetic Teflon ${ }^{\circledR}$-coated stir bar was cooled under a stream of nitrogen and charged with 4,7dibromobenzo[c][1,2,5]thiadiazole (5b) (14.7 g, $50.0 \mathrm{mmol}, 1.00$ equiv) and ethanol $(250 \mathrm{~mL}, 0.20 \mathrm{M})$, and the flask was placed in an ice bath. To the stirred solution was added sodium borohydride (14.2 g, 375 mmol, 7.50 equiv) portionwise over the course of 1 hour, after which the flask was allowed to warm to room temperature. After 18 hours the solvent was removed in vacuo and the crude residue was taken up in ethyl acetate $(300 \mathrm{~mL})$ then saturated aqueous sodium bicarbonate solution $(200 \mathrm{~mL})$ was carefully added. The mixture was placed in a separatory funnel and the aqueous layer was separated then extracted with ethyl acetate $(2 \times 100 \mathrm{~mL})$, then the combined organic extracts were washed with brine and dried over $\mathrm{Na}_{2} \mathrm{SO}_{4}$ then filtered. The solvent was removed in vacuo and the crude residue was dried under high-vacuum 
overnight and carried forward without further purification. To an oven-dried $500 \mathrm{~mL}$ round bottomed flask was added lead (IV) tetraacetate (44.3 g, $100 \mathrm{mmol}, 2.00$ equiv), $100 \mathrm{~mL}$ of anhydrous toluene, and a magnetic Teflon ${ }^{\circledR}$-coated stir bar. The flask was heated to $60{ }^{\circ} \mathrm{C}$ in an oil bath with stirring, then equipped with a slow-addition funnel. To the addition funnel was added the crude ortho-phenylenediamine intermediate in $150 \mathrm{~mL}$ of anhydrous toluene, and the solution was added dropwise over the course of 15 minutes. The reaction mixture was heated to $60^{\circ} \mathrm{C}$ for 2 hours, then was cooled to room temperature and filtered over Celite ${ }^{\circledR}$ and rinsed with dichloromethane. To the mixture was added saturated aqueous sodium bicarbonate solution $(200 \mathrm{~mL})$, and the material was extracted with dichloromethane $(2 \mathrm{x} 100 \mathrm{~mL})$ in a separatory funnel, then the combined organic layers were washed with brine, dried over $\mathrm{Na}_{2} \mathrm{SO}_{4}$, and filtered. The solvent was removed in vacuo and the crude residue was recrystallized from hot toluene to afford the title compound as a pale yellow crystalline solid (7.22 g, 55\%, $27.5 \mathrm{mmol})$.

m.p. $179-181{ }^{\circ} \mathrm{C}$.

$\mathbf{R}_{\mathbf{f}}(90: 10$ hexanes:ethyl acetate $)=0.54$.

${ }^{1} \mathbf{H} \mathbf{N M R}\left(600 \mathrm{MHz}, \mathrm{CDCl}_{3}\right) \delta 7.44(\mathrm{~s}, 2 \mathrm{H})$.

${ }^{13}$ C NMR $\left(151 \mathrm{MHz}, \mathrm{CDCl}_{3}\right) \delta 142.3,113.5,96.5$.

IR (ATR) 3041, 2216, $1543 \mathrm{~cm}^{-1}$.

HRMS (APCI) m/z: [M] $]^{+}$Calcd for $\mathrm{C}_{6} \mathrm{H}_{2} \mathrm{Br}_{2} \mathrm{~N}_{2}$ 259.8585; Found 259.8581.

\section{Preparation and Characterization of Substrates}

\section{General procedure for malonate synthesis - General Procedure B.}

A flame dried $50 \mathrm{~mL} 1$ neck round bottomed flask equipped with a magnetic Teflon ${ }^{\circledR}$-coated stir bar and septum and charged with the appropriate malonate (1.00 equiv), and ethanol (20 mL, $0.50 \mathrm{M})$. The appropriate aldehyde ( 1.00 equiv), acetic acid ( 0.100 equiv), and piperidine ( 0.100 equiv) were added and the flask was equipped with a condenser, placed in an oil bath, and the solution was stirred $100{ }^{\circ} \mathrm{C}$ for 14 hours. The solution was cooled to room temperature and concentrated in vacuo to yield colorless oil. The crude oil was dissolved in ethanol $(0.68 \mathrm{M})$. The solution was cooled to $0{ }^{\circ} \mathrm{C}$ and sodium borohydride $(1.10$ equiv) was added. The mixture was stirred for 1 hour at $25^{\circ} \mathrm{C}$ until TLC suggested full conversion. The mixture was quenched with water $(5 \mathrm{~mL})$ and extracted with ethyl acetate $(3 \times 10 \mathrm{~mL})$. The organic layer was dried with $\mathrm{Na}_{2} \mathrm{SO}_{4}$, and concentrated in vacuo, to yield a colorless oil. The crude material was purified via column chromatography on silica gel. 
<smiles>CCOC(CC)C(Cc1ccc(Br)cc1)OCC</smiles>

Diethyl 2-(4-bromobenzyl)malonate (S3). The title compound was prepared using 4(bromo)benzaldehyde (925 mg, $5.00 \mathrm{mmol}, 1.00$ equiv) following General procedure B and the crude residue was purified by column chromatography on silica gel using 91:9 hexanes:ethyl acetate as the eluent affording the title compound as a colorless oil $(1.23 \mathrm{~g}, 75 \%, 3.75 \mathrm{mmol})$.

$\mathbf{R}_{\mathbf{f}}(91: 9$ hexanes:ethyl acetate $)=0.39$.

${ }^{1}$ H NMR $\left(400 \mathrm{MHz}, \mathrm{CDCl}_{3}\right) \delta 7.42(\mathrm{~d}, J=8.4 \mathrm{~Hz}, 2 \mathrm{H}), 7.10(\mathrm{~d}, J=8.4 \mathrm{~Hz}, 2 \mathrm{H}), 4.18(\mathrm{q}, J=7.1 \mathrm{~Hz}, 4 \mathrm{H})$, $3.62(\mathrm{t}, J=7.8 \mathrm{~Hz}, 1 \mathrm{H}), 3.19(\mathrm{~d}, J=7.8 \mathrm{~Hz}, 2 \mathrm{H}), 1.24(\mathrm{t}, J=7.1 \mathrm{~Hz}, 6 \mathrm{H})$. Spectrum matches previously published data. ${ }^{6}$<smiles>CCOC(=O)C(COC)Cc1ccc(OC)cc1</smiles>

Diethyl 2-(4-methoxybenzyl)malonate (S4). The title compound was prepared using 4(methoxy)benzaldehyde ( $608 \mu \mathrm{L}, \rho=1.12 \mathrm{~g} / \mathrm{mL}, 5.00 \mathrm{mmol}, 1.00$ equiv) following General procedure B and the crude residue was purified by column chromatography on silica gel using 89:11 hexanes:ethyl acetate as the eluent affording the title compound as a colorless oil (785 $\mathrm{mg}, 56 \%, 2.81 \mathrm{mmol})$.

$\mathbf{R}_{\mathbf{f}}(91: 9$ hexanes: ethyl acetate $)=0.32$.

${ }^{1}$ H NMR $\left(400 \mathrm{MHz}, \mathrm{CDCl}_{3}\right) \delta 7.12(\mathrm{~d}, J=8.7 \mathrm{~Hz}, 2 \mathrm{H}), 6.80(\mathrm{~d}, J=8.7,2 \mathrm{H}), 4.15(\mathrm{q}, J=7.1 \mathrm{~Hz}, 4 \mathrm{H}), 3.77$ (s, 3H), $3.60(\mathrm{t}, J=7.8 \mathrm{~Hz}, 1 \mathrm{H}), 3.15(\mathrm{~d}, J=7.8 \mathrm{~Hz}, 2 \mathrm{H}), 1.21(\mathrm{t}, J=7.1 \mathrm{~Hz}, 6 \mathrm{H})$. Spectrum matches previously published data. ${ }^{7}$<smiles>CCOCC(Cc1ccc([N+](=O)[O-])cc1)C(=O)OCC</smiles>

Diethyl 2-(4-nitrobenzyl)malonate (S5). The title compound was prepared using 4-(nitro)benzaldehyde (756 mg, $5.00 \mathrm{mmol}, 1.00$ equiv) following General procedure B and the crude residue was purified by column chromatography on silica gel using 83:17 hexanes:ethyl acetate as the eluent affording the title compound as a colorless solid (957 $\mathrm{mg}, 65 \%, 3.25 \mathrm{mmol})$.

$\mathbf{R}_{\mathbf{f}}(91: 9$ hexanes: ethyl acetate $)=0.22$.

mp. $51-53{ }^{\circ} \mathrm{C}\left(\mathrm{CDCl}_{3}\right)$. 
${ }^{1}$ H NMR $\left(400 \mathrm{MHz}, \mathrm{CDCl}_{3}\right) \delta 8.18(\mathrm{~d}, J=8.8 \mathrm{~Hz}, 2 \mathrm{H}), 7.40(\mathrm{~d}, J=8.8 \mathrm{~Hz}, 2 \mathrm{H}), 4.20(\mathrm{q}, J=7.1 \mathrm{~Hz}, 4 \mathrm{H})$, $3.68(\mathrm{t}, J=7.8 \mathrm{~Hz}, 1 \mathrm{H}), 3.34(\mathrm{~d}, J=7.8 \mathrm{~Hz}, 2 \mathrm{H}), 1.25(\mathrm{t}, J=7.1 \mathrm{~Hz}, 6 \mathrm{H})$. Spectrum matches previously published data. $^{6}$<smiles>CCOCC(Cc1ccc(C)cc1)C(=O)OCC</smiles>

Diethyl 2-(4-nitrobenzyl)malonate (S6). The title compound was prepared using 4-(methyl)benzaldehyde $(590 \mu \mathrm{L}, \rho=1.02 \mathrm{~g} / \mathrm{mL}, 5.00 \mathrm{mmol}, 1.00$ equiv) following General procedure B and the crude residue was purified by column chromatography on silica gel using 91:9 hexanes:ethyl acetate as the eluent affording the title compound as a colorless oil (1.05 g, 80\%, $4.02 \mathrm{mmol})$.

$\mathbf{R}_{\mathbf{f}}(91: 9$ hexanes: ethyl acetate $)=0.43$.

${ }^{1}$ H NMR $\left(400 \mathrm{MHz}, \mathrm{CDCl}_{3}\right) \delta 7.11(\mathrm{~s}, 4 \mathrm{H}), 4.18(\mathrm{q}, J=7.1 \mathrm{~Hz}, 4 \mathrm{H}), 3.64(\mathrm{t}, J=7.8 \mathrm{~Hz}, 1 \mathrm{H}), 3.20(\mathrm{~d}, J=$ $7.8 \mathrm{~Hz}, 2 \mathrm{H}), 2.33(\mathrm{~s}, 3 \mathrm{H}), 1.24(\mathrm{t}, J=7.1 \mathrm{~Hz}, 6 \mathrm{H})$. Spectrum matches previously published data. ${ }^{7}$<smiles>CCOC(=O)C(Cc1c[nH]c2ccccc12)C(=O)OCc1ccccc1</smiles>

Diethyl 2-((1-(tert-butoxycarbonyl)-1H-indol-3-yl)methyl)malonate (S7). To a $250 \mathrm{~mL}$ round-bottomed flask was added diethyl 2-((1H-indol-3-yl)methyl)malonate $(5.78 \mathrm{~g}, 20.0 \mathrm{mmol})$ in $100 \mathrm{~mL}$ of dichloromethane at $25{ }^{\circ} \mathrm{C}$. To the stirred reaction mixture was added di-tert-butyl pyrocarbonate $(5.24 \mathrm{~g}$, $24.0 \mathrm{mmol}$ ), then 4-(dimethylamino)pyridine $(244 \mathrm{mg}, 2.00 \mathrm{mmol})$ and the solution was stirred at room temperature for $18 \mathrm{~h}$, after which the solvent was concentrated in vacuo and the crude residue was purified via column chromatography on silica gel using 98:2 to 95:5 hexanes:ethyl acetate as the eluent to afford the title compound as a colorless oil (7.42 $\mathrm{g}, 95 \%, 19.1 \mathrm{mmol})$.

$\mathbf{R}_{\mathbf{f}}(95: 5$ hexanes:ethyl acetate $)=0.13$.

${ }^{1}$ H NMR $\left(600 \mathrm{MHz}, \mathrm{CDCl}_{3}\right) \delta 8.11(\mathrm{~s}, 1 \mathrm{H}), 7.54(\mathrm{~d}, \mathrm{~J}=7.8 \mathrm{~Hz}, 1 \mathrm{H}), 7.47-7.36(\mathrm{~m}, 1 \mathrm{H}), 7.31(\mathrm{~m}, 1 \mathrm{H}), 7.25$ (m, 1H), $4.18(\mathrm{q}, J=7.2 \mathrm{~Hz}, 4 \mathrm{H}) 3.75$ (t, $J=7.7 \mathrm{~Hz}, 1 \mathrm{H}), 3.31$ (d, $J=7.7 \mathrm{~Hz}, 2 \mathrm{H}), 1.65$ (s, 9H), 1.23 (t, $J$ $=7.2 \mathrm{~Hz}, 6 \mathrm{H})$.

${ }^{13} \mathbf{C}$ NMR $\left(151 \mathrm{MHz}, \mathrm{CDCl}_{3}\right) \delta 169.1,124.6,123.7,122.6,118.9,117.0,115.4,83.7,61.7,52.2,28.3,24.3$, 14.2.

IR (ATR) 2979, 2953, 1727, 1452, 1367, 1239, 1153, 1048, 857, $746 \mathrm{~cm}^{-1}$. 
HRMS (APCI) m/z: [M+H] ${ }^{+}$Calcd for $\mathrm{C}_{17} \mathrm{H}_{20} \mathrm{NO}_{6}$ 334.1291; Found 334.1284 (note: Calcd and observed masses are reflective of the carbamic acid without the tert-butyl group).<smiles>CCOC(=O)C(=Cc1cccc(OC)c1)OCC</smiles>

Diethyl 2-(3-methoxybenzylidene)malonate (S8). A flame dried $50 \mathrm{~mL} 1$ neck round bottomed flask equipped with a magnetic Teflon ${ }^{\circledR}$-coated stir bar and septum and charged with diethyl malonate $(1.5 \mathrm{~mL}$, $\rho=1.05 \mathrm{~g} / \mathrm{mL}, 9.8 \mathrm{mmol}, 1.0$ equiv), and ethanol (20 mL, $0.5 \mathrm{M})$. meta-Anisaldehyde $(1.2 \mathrm{~mL}, \rho=1.117$ $\mathrm{g} / \mathrm{mL}, 9.8 \mathrm{mmol}, 1.0$ equiv), acetic acid $(60 \mu \mathrm{L}, \rho=1.05 \mathrm{~g} / \mathrm{mL}, 1.0 \mathrm{mmol}, 0.10$ equiv), and piperidine (100 $\mu \mathrm{L}, \rho=0.862 \mathrm{~g} / \mathrm{mL}, 1.0 \mathrm{mmol}, 0.10$ equiv) were added. The flask was equipped with a reflux condenser and the solution was stirred $100{ }^{\circ} \mathrm{C}$ for 14 hours. The solution was then cooled to room temperature and concentrated in vacuo to yield colorless oil. The crude material was purified via column chromatography on silica gel using 95:5 hexanes:ethyl acetate as the eluent to afford the title compound as a colorless oil $(1.9 \mathrm{~g}, 68 \%, 6.8 \mathrm{mmol})$.

$\mathbf{R}_{\mathbf{f}}(95: 5$ hexanes:ethyl acetate $)=0.12$.

${ }^{1}$ H NMR $\left(400 \mathrm{MHz}, \mathrm{CDCl}_{3}\right) \delta 7.70(\mathrm{~s}, 1 \mathrm{H}), 7.33-7.23(\mathrm{~m}, 2 \mathrm{H}), 7.04(\mathrm{~d}, J=7.7 \mathrm{~Hz}, 1 \mathrm{H}), 6.99(\mathrm{t}, J=2.1$ $\mathrm{Hz}, 1 \mathrm{H}), 6.94(\mathrm{dd}, J=8.2,2.6 \mathrm{~Hz}, 1 \mathrm{H}), 4.36-4.28(\mathrm{~m}, 4 \mathrm{H}), 3.80(\mathrm{~s}, 3 \mathrm{H}), 1.35-1.28(\mathrm{~m}, 6 \mathrm{H})$. Spectra matches previously published data. ${ }^{8}$<smiles>CCOC(=O)C(Cc1cccc(OC)c1)OCC</smiles>

Diethyl 2-(3-methoxybenzyl)malonate (S9). A $20 \mathrm{~mL}$ vial equipped with a septum and magnetic Teflon ${ }^{\circledR}$ coated stir bar charged with diethyl 2-(3-methoxybenzylidene)malonate (S8) (1.9 g, 6.8 mmol, 1.0 equiv) and ethanol $(10 \mathrm{~mL}, 0.68 \mathrm{M})$. The solution was cooled to $0{ }^{\circ} \mathrm{C}$ and sodium borohydride $(0.28 \mathrm{~g}, 7.5 \mathrm{mmol}$, 1.1 equiv) was added. The solution was stirred for 1 hour at $25^{\circ} \mathrm{C}$ until TLC suggested full conversion. The mixture was quenched with water $(5 \mathrm{~mL})$ and extracted with ethyl acetate $(3 \times 10 \mathrm{~mL})$. The organic layer was dried with $\mathrm{Na}_{2} \mathrm{SO}_{4}$, and concentrated in vacuo, to yield a colorless oil. The crude material was purified via column chromatography on silica gel using 90:10 hexanes:ethyl acetate as the eluent. This procedure afforded the title compound as a colorless oil $(1.1 \mathrm{~g}, 58 \%, 3.9 \mathrm{mmol})$.

$\mathbf{R}_{\mathbf{f}}(90: 10$ hexanes:ethyl acetate $)=0.28$. 
${ }^{1}$ H NMR $\left(400 \mathrm{MHz}, \mathrm{CDCl}_{3}\right) \delta 7.19(\mathrm{t}, J=8.2 \mathrm{~Hz}, 1 \mathrm{H}), 6.83-6.71(\mathrm{~m}, 3 \mathrm{H}), 4.17(\mathrm{q}, J=7.2 \mathrm{~Hz}, 4 \mathrm{H}), 3.78$ (s, 3H), $3.64(\mathrm{dd}, J=8.5,7.1 \mathrm{~Hz}, 1 \mathrm{H}), 3.19$ (d, $J=7.9 \mathrm{~Hz}, 2 \mathrm{H}), 1.22$ (t, $J=7.1 \mathrm{~Hz}, 6 \mathrm{H})$. Spectra matches previously published data. ${ }^{7}$

\section{Preparation and Characterization of Amine Addition Products}

\section{1) General procedure for the addition of amine nucleophiles to bis(2-haloacrylonitrile) - General} Procedure C.

To a $4 \mathrm{~mL}$ dram vial equipped with magnetic Teflon ${ }^{\circledR}$-coated stir bar was added bis(2-chloroacrylonitrile) (3a) (34.6 mg, $200 \mu \mathrm{mol}, 1.00$ equiv) or bis(2-bromoacrylonitrile) (3b) (52.4 mg, $0.2 \mathrm{mmol}, 1.00$ equiv). The vials were capped then evacuated and backfilled with $\mathrm{N}_{2}$, then THF $(0.6 \mathrm{~mL}, 0.33 \mathrm{M})$ was added. The stirred homogeneous solution was charged with an amine (700 $\mu \mathrm{mol}, 3.50$ equiv), and the reaction was stirred at $23{ }^{\circ} \mathrm{C}$ for 1 hour. The solvent was then removed in vacuo and the products were isolated by silica gel flash column chromatography.

2) General procedure for the addition of amine hydrochloride nucleophiles to bis(2-haloacrylonitrile) - General Procedure D.

To a $4 \mathrm{~mL}$ dram vial equipped with magnetic Teflon ${ }^{\circledR}$-coated stir bar was added bis(2-chloroacrylonitrile) (3a) (34.6 mg, $200 \mu \mathrm{mol}, 1.00$ equiv) or bis(2-bromoacrylonitrile) (3b) (52.4 mg, $0.2 \mathrm{mmol}, 1.00$ equiv). To the vial was then added cesium carbonate (326 mg, $1.00 \mathrm{mmol}, 5.00$ equiv) and amine hydrochloride salt $\left(0.7 \mathrm{mmol}, 3.50\right.$ equiv). The vials were capped then evacuated and placed under $\mathrm{N}_{2}$, then THF (2.00 $\mathrm{mL}, 0.1 \mathrm{M})$ was added, and the reaction was stirred at $23{ }^{\circ} \mathrm{C}$ for $18-24$ hours. Deionized water $(2.00 \mathrm{~mL})$ was added, and the mixture was extracted with ethyl acetate $(3 \times 1.0 \mathrm{~mL})$ then the combined organic layers were washed with brine. The combined organic layers were dried over $\mathrm{Na}_{2} \mathrm{SO}_{4}$ then the solvent was removed in vacuo and the products were isolated by silica gel flash column chromatography.

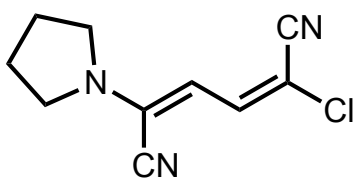

(2E,4E)-2-Chloro-5-(pyrrolidin-1-yl)hexa-2,4-dienedinitrile (7a): The title compound was prepared from bis(2-chloroacrylonitrile) (3a) (172 mg, $1.00 \mathrm{mmol}, 1.00$ equiv) and pyrrolidine $(57.5 \mu \mathrm{L}, \rho=0.866$ $\mathrm{g} / \mathrm{mL}, 700 \mu \mathrm{mol}, 3.50$ equiv) following General Procedure $\mathrm{C}$, on $1.00 \mathrm{mmol}$ scale. The crude material was purified via column chromatography on silica gel using 91:9 hexanes:ethyl acetate as the eluent to afford the title compound as a yellow solid (191 mg, 92\%, $184 \mu \mathrm{mol})$. 
$\mathbf{R}_{\mathbf{f}}(83: 17$ hexanes:ethyl acetate $)=0.39$.

m.p. ${ }^{113}-115^{\circ} \mathrm{C}\left(\mathrm{CDCl}_{3}\right)$.

${ }^{1}$ H NMR $\left(600 \mathrm{MHz}, \mathrm{CDCl}_{3}\right) \delta 7.25(\mathrm{~d}, J=12.2 \mathrm{~Hz}, 1 \mathrm{H}), 5.59(\mathrm{~d}, J=12.2 \mathrm{~Hz}, 1 \mathrm{H}), 3.46-3.40(\mathrm{~m}, 4 \mathrm{H})$, 2.06-2.01 (m, 4H).

${ }^{13}$ C NMR $\left(151 \mathrm{MHz}, \mathrm{CDCl}_{3}\right) \delta 142.5,126.5,115.5,112.8,103.7,94.1,49.9,25.3$.

IR (ATR) 3046, 2977, 2865, 2235, 2197, 1579, $1558 \mathrm{~cm}^{-1}$.

HRMS (APCI) m/z: [M] Calcd for $\mathrm{C}_{10} \mathrm{H}_{10} \mathrm{ClN}_{3}$ 207.0563; Found 207.0558.

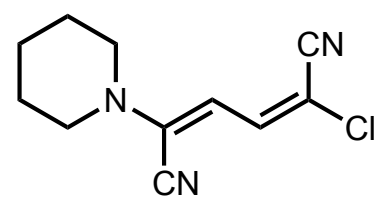

(2E,4E)-2-Chloro-5-(piperidin-1-yl)hexa-2,4-dienedinitrile (8a): The title compound was prepared from bis(2-chloroacrylonitrile) (3a) and piperidine $(69.1 \mu \mathrm{L}, \rho=0.862 \mathrm{~g} / \mathrm{mL}, 700 \mu \mathrm{mol}, 3.50$ equiv) following General Procedure C. The crude residue was purified via column chromatography on silica gel using 91:9 hexanes:ethyl acetate as the eluent to afford the title compound as a yellow solid (42.2 $\mathrm{mg}, 95 \%, 190 \mu \mathrm{mol})$.

$\mathbf{R}_{\mathbf{f}}(83: 17$ hexanes:ethyl acetate $)=0.46$.

m.p. $69-71{ }^{\circ} \mathrm{C}\left(\mathrm{CDCl}_{3}\right)$.

${ }^{1}$ H NMR $\left(600 \mathrm{MHz}, \mathrm{CDCl}_{3}\right) \delta 7.27(\mathrm{~d}, J=12.1 \mathrm{~Hz}, 1 \mathrm{H}), 5.88(\mathrm{~d}, J=12.1 \mathrm{~Hz}, 1 \mathrm{H}), 3.37-3.33(\mathrm{~m}, 4 \mathrm{H})$, $1.70-1.64(\mathrm{~m}, 6 \mathrm{H})$.

${ }^{13} \mathbf{C}$ NMR $\left(151 \mathrm{MHz}, \mathrm{CDCl}_{3}\right) \delta 142.8,129.7,115.3,112.7,105.7,96.1,49.7,25.2,23.8$.

IR (ATR) 2941, 2857, 2226, 2204, 1574, $1553 \mathrm{~cm}^{-1}$.

HRMS (APCI) m/z: Calcd for $\mathrm{C}_{11} \mathrm{H}_{12} \mathrm{ClN}_{3}[\mathrm{M}]^{+}:$221.0720; Found 221.0716.

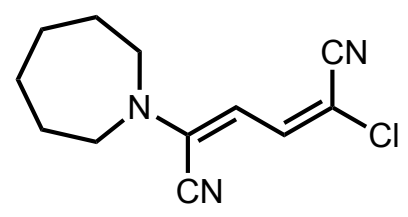

(2E,4E)-2-(Azepan-1-yl)-5-chlorohexa-2,4-dienedinitrile (9a): The title compound was prepared from bis(2-chloroacrylonitrile) (3a) and azepane $(78.9 \mu \mathrm{L}, \rho=0.880 \mathrm{~g} / \mathrm{mL}, 700 \mu \mathrm{mol}, 3.50$ equiv) following General Procedure C. The crude residue was purified via column chromatography on silica gel using 91:9 
hexanes:ethyl acetate as the eluent to afford the title compound as a yellow oil that solidified on standing (43.5 mg, 92\%, $184 \mu \mathrm{mol})$.

$\mathbf{R}_{\mathbf{f}}(83: 17$ hexanes:ethyl acetate $)=0.43$.

m.p. $55-57{ }^{\circ} \mathrm{C}\left(\mathrm{CDCl}_{3}\right)$.

${ }^{1}$ H NMR (600 MHz, $\left.\mathrm{CDCl}_{3}\right) 7.25(\mathrm{~d}, J=12.1 \mathrm{~Hz}, 1 \mathrm{H}), 5.73(\mathrm{~d}, J=12.1 \mathrm{~Hz}, 1 \mathrm{H}), 3.50-3.46(\mathrm{~m}, 4 \mathrm{H}), 1.83-$ $1.77(\mathrm{~m}, 4 \mathrm{H}), 1.62-1.58(\mathrm{~m}, 4 \mathrm{H})$.

${ }^{13}$ C NMR $\left(151 \mathrm{MHz}, \mathrm{CDCl}_{3}\right) \delta 143.1,129.1,115.6,112.6,103.1,94.2,51.8,27.8,27.1$.

IR (ATR) 2928, 2856, 2229, 2202, 1572, $1551 \mathrm{~cm}^{-1}$.

HRMS (APCI) m/z: [M] $]^{+}$Calcd for $\mathrm{C}_{12} \mathrm{H}_{14} \mathrm{ClN}_{3} 235.0876$; Found 235.0871.<smiles>N#C/C(Cl)=C\C=C(/C#N)N1CCOCC1</smiles>

(2E,4E)-2-Chloro-5-morpholinohexa-2,4-dienedinitrile (10a): The title compound was prepared from bis(2-chloroacrylonitrile) (3a) and morpholine $(60.4 \mu \mathrm{L}, \rho=1.01 \mathrm{~g} / \mathrm{mL}, 700 \mu \mathrm{mol}, 3.50$ equiv) following General Procedure C. The crude residue was purified via column chromatography on silica gel using 83:17 hexanes:ethyl acetate as the eluent to afford the title compound as a yellow solid ( $40.1 \mathrm{mg}, 90 \%, 180 \mu \mathrm{mol})$. $\mathbf{R}_{\mathbf{f}}(83: 17$ hexanes: ethyl acetate $)=0.25$.

m.p. $147-149^{\circ} \mathrm{C}\left(\mathrm{CDCl}_{3}\right)$.

${ }^{1} \mathbf{H}$ NMR $\left(600 \mathrm{MHz}, \mathrm{CDCl}_{3}\right) \delta 7.27(\mathrm{~d}, J=12.1 \mathrm{~Hz}, 1 \mathrm{H}), 5.94(\mathrm{~d}, J=12.1 \mathrm{~Hz}, 1 \mathrm{H}), 3.79(\mathrm{t}, J=4.8 \mathrm{~Hz}$, 4H), $3.31(\mathrm{t}, J=4.8 \mathrm{~Hz}, 4 \mathrm{H})$.

${ }^{13}$ C NMR $\left(151 \mathrm{MHz}, \mathrm{CDCl}_{3}\right) \delta 141.9,129.8,114.7,112.3,107.4,98.6,65.8,48.3$.

IR (ATR) 2984, 2904, 2883, 2226, 2211, 1591, $1553 \mathrm{~cm}^{-1}$.

HRMS (APCI) m/z: [M] $]^{+}$Calcd for $\mathrm{C}_{10} \mathrm{H}_{10} \mathrm{ClN}_{3} \mathrm{O}$ 223.0512; Found 223.0509.<smiles>N#C/C(Cl)=C\C=C(/C#N)N1CCSCC1</smiles>

(2E,4E)-2-Chloro-5-(4-methylpiperazin-1-yl)hexa-2,4-dienedinitrile (11a): The title compound was prepared from bis(2-chloroacrylonitrile) (3a) and thiomorpholine $(70.1 \mu \mathrm{L}, \rho=1.03 \mathrm{~g} / \mathrm{mL}, 700 \mu \mathrm{mol}, 3.50$ 
equiv) following General Procedure C. The crude residue was purified via column chromatography on silica gel using 83:17 hexanes:ethyl acetate as the eluent to afford the title compound as a yellow oil that solidified on standing (42.7 mg, 90\% $180 \mu \mathrm{mol})$.

$\mathbf{R}_{\mathbf{f}}(83: 17$ hexanes: ethyl acetate $)=0.45$.

m.p. $108-110^{\circ} \mathrm{C}\left(\mathrm{CDCl}_{3}\right)$.

${ }^{1}$ H NMR $\left(600 \mathrm{MHz}, \mathrm{CDCl}_{3}\right) \delta 7.27(\mathrm{~d}, J=12.0 \mathrm{~Hz}, 1 \mathrm{H}), 5.91(\mathrm{~d}, J=12.0 \mathrm{~Hz}, 1 \mathrm{H}), 3.74-3.70(\mathrm{~m}, 4 \mathrm{H})$, $2.72-2.68(\mathrm{~m}, 4 \mathrm{H})$.

${ }^{13}$ C NMR $\left(151 \mathrm{MHz}, \mathrm{CDCl}_{3}\right) \delta 142.2,128.4,114.9,112.4,106.9,97.6,51.3,26.4$.

IR (ATR) 3071, 2920, 2852, 2229, 2205, 1574, $1551 \mathrm{~cm}^{-1}$.

HRMS (APCI) m/z: [M] $]^{+}$Calcd for $\mathrm{C}_{10} \mathrm{H}_{10} \mathrm{ClN}_{3} \mathrm{~S} 239.0284$; Found 239.0279.<smiles>CN1CCN(/C(C#N)=C/C=C(Cl)Cl)CC1</smiles>

(2E,4E)-2-Chloro-5-(4-oxopiperidin-1-yl)hexa-2,4-dienedinitrile (12a): The title compound was prepared from bis(2-chloroacrylonitrile) (3a) and 1-methylpiperazine $(77.7 \mu \mathrm{L}, \rho=0.903 \mathrm{~g} / \mathrm{mL}, 700 \mu \mathrm{mol}$, 3.50 equiv) following General Procedure C. The crude residue was purified via column chromatography on silica gel using 99:1 dichloromethane:methanol as the eluent to afford the title compound as a yellow solid (46.5 mg, 98\%, $196 \mu \mathrm{mol})$.

$\mathbf{R}_{\mathbf{f}}(50: 50$ hexanes:ethyl acetate $)=0.12$.

m.p. ${ }^{139}-141^{\circ} \mathrm{C}\left(\mathrm{CDCl}_{3}\right)$.

${ }^{1} \mathbf{H}$ NMR $\left(600 \mathrm{MHz}, \mathrm{CDCl}_{3}\right) \delta 7.27(\mathrm{~d}, J=12.0 \mathrm{~Hz}, 1 \mathrm{H}), 5.91(\mathrm{~d}, J=12.0 \mathrm{~Hz}, 1 \mathrm{H}), 3.39-3.33(\mathrm{~m}, 4 \mathrm{H})$, $2.55-2.46(\mathrm{~m}, 4 \mathrm{H}), 2.34(\mathrm{~s}, 3 \mathrm{H})$.

${ }^{13}$ C NMR $\left(151 \mathrm{MHz}, \mathrm{CDCl}_{3}\right) \delta 142.3,129.5,114.9,112.5,106.8,97.6,53.7,48.1,45.9$.

IR (ATR) 3056, 2934, 2805, 2222, 2210, 1745, 1582, $1552 \mathrm{~cm}^{-1}$.

HRMS (APCI) m/z: $[\mathrm{M}+\mathrm{H}]^{+}$Calcd for $\mathrm{C}_{11} \mathrm{H}_{13} \mathrm{ClN}_{4} 236.0829$; Found 236.0823.<smiles>N#C/C(Cl)=C\C=C(/C#N)N1CCC(=O)CC1</smiles> 
(2E,4E)-2-Chloro-5-(dimethylamino)hexa-2,4-dienedinitrile (13a): The title compound was prepared from bis(2-chloroacrylonitrile) (3a) and 4-piperidone hydrochloride (94.9 mg, $700 \mu \mathrm{mol}, 3.50$ equiv) following General Procedure D. The crude residue was purified via column chromatography on silica gel using 75:25 hexanes:ethyl acetate as the eluent to afford the title compound as a yellow solid (30.9 $\mathrm{mg}$, $66 \%, 132 \mu \mathrm{mol})$.

$\mathbf{R}_{\mathbf{f}}(75: 25$ hexanes:ethyl acetate $)=0.36$.

m.p. ${ }^{113}-115^{\circ} \mathrm{C}\left(\mathrm{CDCl}_{3}\right)$.

${ }^{1} \mathbf{H}$ NMR $\left(600 \mathrm{MHz}, \mathrm{CDCl}_{3}\right) \delta 7.28(\mathrm{~d}, J=12.0 \mathrm{~Hz}, 1 \mathrm{H}), 5.96(\mathrm{~d}, J=12.0 \mathrm{~Hz}, 1 \mathrm{H}), 3.73(\mathrm{t}, J=6.3 \mathrm{~Hz}$, $4 \mathrm{H}), 2.61(\mathrm{t}, J=6.3 \mathrm{~Hz}, 4 \mathrm{H})$.

${ }^{13}$ C NMR (151 MHz, $\left.\mathrm{CDCl}_{3}\right) \delta 204.6,141.9,128.1,114.7,107.34,98.4,47.0,40.0$.

IR (ATR) 2920, 2228, 2206, 1721, 1579, $1557 \mathrm{~cm}^{-1}$.

HRMS (APCI) m/z: [M] $]^{+}$Calcd for $\mathrm{C}_{11} \mathrm{H}_{10} \mathrm{ClN}_{3} \mathrm{O}$ 235.0512; Found 235.0507.<smiles>N#C/C(Cl)=C\C=C(/C#N)N1CCc2ccccc2C1</smiles>

(2E,4E)-2-chloro-5-(3,4-dihydroisoquinolin-2(1H)-yl)hexa-2,4-dienedinitrile $\quad(14 a): \quad$ The title compound was prepared from bis(2-chloroacrylonitrile) (3a) and 1,2,3,4-tetrahydroisoquinoline (88.8 $\mu \mathrm{L}$, $\rho=1.05 \mathrm{~g} / \mathrm{mL}, 700 \mu \mathrm{mol}, 3.50$ equiv) following General Procedure C. The crude residue was purified via column chromatography on silica gel using 50:50 hexanes:dichloromethane as the eluent to afford the title compound as a yellow solid $(46.1 \mathrm{mg}, 86 \%, 172 \mu \mathrm{mol})$.

$\mathbf{R}_{\mathbf{f}}(83: 17$ hexanes:ethyl acetate $)=0.54$.

m.p. $113-115^{\circ} \mathrm{C}\left(\mathrm{CDCl}_{3}\right)$.

${ }^{1}$ H NMR $\left(600 \mathrm{MHz}, \mathrm{CDCl}_{3}\right) \delta 7.30(\mathrm{~d}, J=12.0 \mathrm{~Hz}, 1 \mathrm{H}), 7.29-7.24(\mathrm{~m}, 2 \mathrm{H}), 7.21-7.17(\mathrm{~m}, 2 \mathrm{H}), 5.92(\mathrm{~d}$, $J=12.0 \mathrm{~Hz}, 1 \mathrm{H}), 4.47(\mathrm{~s}, 2 \mathrm{H}), 3.72(\mathrm{t}, J=5.9 \mathrm{~Hz}, 2 \mathrm{H}), 2.99(\mathrm{t}, J=5.9 \mathrm{~Hz}, 2 \mathrm{H})$.

${ }^{13}$ C NMR $\left(151 \mathrm{MHz}, \mathrm{CDCl}_{3}\right) \delta 142.5,133.7,131.0,128.9,128.6,127.6,127.1,126.4,115.2,112.6,105.1$, $96.4,49.7,47.0,28.8$.

IR (ATR) 3056, 2919, 2853, 2227, 2207, 1576, $1553 \mathrm{~cm}^{-1}$.

HRMS (APCI) m/z: [M] $]^{+}$Calcd for $\mathrm{C}_{15} \mathrm{H}_{12} \mathrm{ClN}_{3}$ 269.0720; Found 269.0717. 


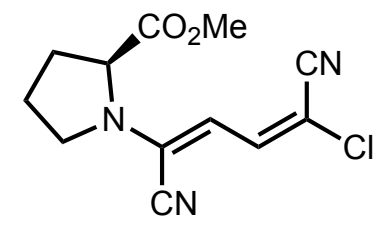

(2E,4E)-2-Chloro-5-thiomorpholinohexa-2,4-dienedinitrile (15a): The title compound was prepared from bis(2-chloroacrylonitrile) (3a) and $L$-proline methyl ester hydrochloride (116 mg, $700 \mu \mathrm{mol}, 3.50$ equiv) following General Procedure D. The crude residue was purified via column chromatography on silica gel using 80:20 hexanes:ethyl acetate as the eluent to afford the title compound as a yellow oil (24.7 mg, 47\%, $94 \mu \mathrm{mol})$.

$\mathbf{R}_{\mathbf{f}}(50: 50$ hexanes: ethyl acetate $)=0.62$.

${ }^{1}$ H NMR $\left(600 \mathrm{MHz}, \mathrm{CDCl}_{3}\right) \delta 7.20(\mathrm{~d}, J=12.1 \mathrm{~Hz}, 1 \mathrm{H}), 5.70(\mathrm{~d}, J=12.1 \mathrm{~Hz}, 1 \mathrm{H}), 4.43(\mathrm{dd}, J=8.5,2.6$ $\mathrm{Hz}, 1 \mathrm{H}), 3.79$ (s, 3H), 3.57-3.51 (m, 1H), 3.44-3.38 (m, 1H), 2.36-2.28 (m, 1H), 2.26-2.20 (m, 1H), 2.13$2.04(\mathrm{~m}, 2 \mathrm{H})$.

${ }^{13}$ C NMR $\left(151 \mathrm{MHz}, \mathrm{CDCl}_{3}\right) \delta 171.5,141.9,125.7,114.9,112.4,105.7,96.2,62.5,52.9,49.5,30.3,23.4$. IR (ATR) 2955, 2876, 2234, 2206, 1744, 1582, $1559 \mathrm{~cm}^{-1}$.

HRMS (APCI) m/z: [M] $]^{+}$: Calcd for $\mathrm{C}_{12} \mathrm{H}_{12} \mathrm{ClN}_{3} \mathrm{O}_{2}$ 265.0618; Found 265.0617.<smiles>CN(Cc1ccccc1)/C(C#N)=C/C=C(\Cl)C#N</smiles>

(2E,4E)-2-(Benzyl(methyl)amino)-5-chlorohexa-2,4-dienedinitrile (16a): The title compound was prepared from bis(2-chloroacrylonitrile) (3a) and $N$-methylbenzylamine $(90.3 \mu \mathrm{L}, \rho=0.939 \mathrm{~g} / \mathrm{mL}, 700$ $\mu$ mol, 3.50 equiv) following General Procedure C. The crude residue was purified via column chromatography on silica gel using 91:9 hexanes:ethyl acetate as the eluent to afford of the title compound as a yellow/orange oil (44.3 $\mathrm{mg}, 86 \%, 172 \mu \mathrm{mol})$.

$\mathbf{R}_{\mathbf{f}}(83: 17$ hexanes: ethyl acetate $)=0.46$.

${ }^{1}$ H NMR $\left(600 \mathrm{MHz}, \mathrm{CDCl}_{3}\right) \delta$ 7.44-7.32 (m, 3H), $7.28(\mathrm{~d}, J=12.1 \mathrm{~Hz}, 1 \mathrm{H}), 7.25-7.17(\mathrm{~m}, 2 \mathrm{H}), 5.80(\mathrm{~d}, J$ $=12.1 \mathrm{~Hz}, 1 \mathrm{H}), 4.60(\mathrm{~s}, 2 \mathrm{H}), 2.92(\mathrm{~s}, 3 \mathrm{H})$.

${ }^{13}$ C NMR (151 MHz, $\left.\mathrm{CDCl}_{3}\right) \delta 142.6,135.2,129.3$ (two peaks), 128.6, 127.5, 115.1, 112.8, 105.3, 96.1, $58.2,37.9$.

IR (ATR) 3065, 3030, 2928, 2230, 2205, 1577, $1558 \mathrm{~cm}^{-1}$. 
HRMS (APCI) m/z: [M] $]^{+}$Calcd for $\mathrm{C}_{14} \mathrm{H}_{12} \mathrm{ClN}_{3}$ 257.0720; Found 257.0718.<smiles>CN(CCc1ccccc1)/C(C#N)=C/C=C(\Cl)C#N</smiles>

(2E,4E)-2-Chloro-5-(methyl(phenethyl)amino)hexa-2,4-dienedinitrile (17a): The title compound was prepared from bis(2-chloroacrylonitrile) (3a) and $N$-methylphenethylamine $(102 \mu \mathrm{L}, \rho=0.930 \mathrm{~g} / \mathrm{mL}, 700$ $\mu$ mol, 3.50 equiv) following General Procedure C. The crude residue was purified via column chromatography on silica gel using 91:9 hexanes:ethyl acetate as the eluent to afford of the title compound as a yellow solid $(51.3 \mathrm{mg}, 94 \%, 188 \mu \mathrm{mol})$.

$\mathbf{R}_{\mathbf{f}}(83: 17$ hexanes:ethyl acetate $)=0.38$.

m.p. $77-79{ }^{\circ} \mathrm{C}\left(\mathrm{CDCl}_{3}\right)$.

${ }^{1}$ H NMR (600 MHz, $\left.\mathrm{CDCl}_{3}\right) \delta$ 7.35-7.31 (m, 2H), 7.29-7.25 (m, 1H), 7.20 (d, J=12.1 Hz, 1H), 7.19-7.16 (m, 2H), $5.66(\mathrm{~d}, J=12.1 \mathrm{~Hz}, 1 \mathrm{H}), 3.67$ (t, $J=7.2 \mathrm{~Hz}, 2 \mathrm{H}), 2.89$ (t, $J=7.2 \mathrm{~Hz}, 2 \mathrm{H}), 2.89(\mathrm{~s}, 3 \mathrm{H})$.

${ }^{13}$ C NMR $\left(151 \mathrm{MHz}, \mathrm{CDCl}_{3}\right) \delta 142.6,137.3,129.1,129.0,128.9,127.3,115.3,112.4,104.5,95.5,56.7$, 38.6, 34.9.

IR (ATR) 3064, 3027, 2937, 2230, 2203, 1577, $1558 \mathrm{~cm}^{-1}$.

HRMS (APCI) m/z: [M] $]^{+}$Calcd for $\mathrm{C}_{15} \mathrm{H}_{14} \mathrm{ClN}_{3} 271.0876$; Found 271.0875.<smiles>CN(C)/C(C#N)=C/C=C(\Cl)C#N</smiles>

((1E,3E)-Methyl-4-chloro-1,4-dicyanobuta-1,3-dien-1-yl)-L-prolinate (18a): The title compound was prepared from bis(2-chloroacrylonitrile) (3a) and dimethylamine hydrochloride (57.1 mg, $700 \mu \mathrm{mol}, 3.50$ equiv) following General Procedure D. The crude residue was purified via column chromatography on silica gel using 83:17 hexanes:ethyl acetate as the eluent to afford the title compound as a yellow solid (31.9 $\mathrm{mg}, 88 \%, 176 \mu \mathrm{mol})$.

$\mathbf{R}_{\mathbf{f}}(83: 17$ hexanes:ethyl acetate $)=0.23$.

m.p. $99-101^{\circ} \mathrm{C}\left(\mathrm{CDCl}_{3}\right)$.

${ }^{1}$ H NMR $\left(600 \mathrm{MHz}, \mathrm{CDCl}_{3}\right) 7.22(\mathrm{~d}, J=12.1 \mathrm{~Hz}, 1 \mathrm{H}), 5.70(\mathrm{~d}, J=12.1 \mathrm{~Hz}, 1 \mathrm{H}), 3.06(\mathrm{~s}, 6 \mathrm{H})$.

${ }^{13}$ C NMR (151 MHz, $\left.\mathrm{CDCl}_{3}\right) \delta 142.6$ 129.7, 115.3, 112.5, 104.6, 95.4, 40.9. 
IR (ATR) 2923, 2231, 2207, 1590, $1565 \mathrm{~cm}^{-1}$.

HRMS (APCI) m/z: [M] $]^{+}$Calcd for $\mathrm{C}_{8} \mathrm{H}_{8} \mathrm{ClN}_{3}$ 181.0407; Found 181.0401.

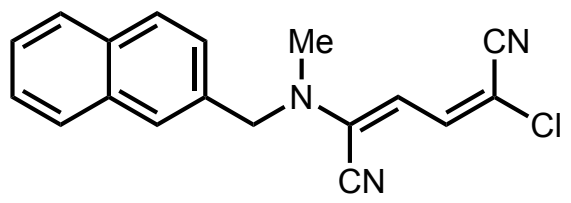

(2E,4E)-2-Chloro-5-(methyl(naphthalen-2-ylmethyl)amino)hexa-2,4-dienedinitrile (19a): The title compound was prepared from bis(2-chloroacrylonitrile) (3a) and $N$-methyl-1-(naphthalen-2yl)methanamine (145 mg, $700 \mu \mathrm{mol}, 3.50$ equiv) following General Procedure D. The crude residue was purified via column chromatography on silica gel using 91:9 hexanes:ethyl acetate as the eluent to afford the title compound as a yellow/orange crystalline solid $(57.5 \mathrm{mg}, 93 \%, 186 \mu \mathrm{mol})$.

$\mathbf{R}_{\mathbf{f}}(83: 17$ hexanes: ethyl acetate $)=0.40$.

m.p. $128-130{ }^{\circ} \mathrm{C}\left(\mathrm{CDCl}_{3}\right)$.

${ }^{1} \mathbf{H}$ NMR $\left(600 \mathrm{MHz}, \mathrm{CDCl}_{3}\right)$ 7.89-7.83 (m, 3H), 7.65 (s, 1H), 7.55-7.50 (m, 2H), 7.32-7.29 (m, 1H), 7.31 $(\mathrm{d}, J=12.0 \mathrm{~Hz}, 1 \mathrm{H}), 5.92(\mathrm{~d}, J=12.0 \mathrm{~Hz}, 1 \mathrm{H}), 4.76(\mathrm{~s}, 2 \mathrm{H}), 2.96(\mathrm{~s}, 3 \mathrm{H})$.

${ }^{13}$ C NMR $\left(151 \mathrm{MHz}, \mathrm{CDCl}_{3}\right) \delta 142.6,133.4,133.2,132.6,129.4,129.3,128.0,127.9,126.9,126.8,126.7$, $124.9,115.1,112.8,105.4,96.3,58.5,37.9$.

IR (ATR) 3055, 2923, 2853, 2229, 2205, 1579, $1559 \mathrm{~cm}^{-1}$.

HRMS (APCI) m/z: [M] $]^{+}$Calcd for $\mathrm{C}_{18} \mathrm{H}_{14} \mathrm{ClN}_{3}$ 307.0876; Found 307.0871.<smiles>N#C/C(Br)=C\C=C(/Br)N1CCCC1</smiles>

(2E,4E)-2-Bromo-5-(pyrrolidin-1-yl)hexa-2,4-dienedinitrile (7b): The title compound was prepared from bis(2-bromoacrylonitrile) (3b) (260 mg, $1 \mathrm{mmol}, 1$ equiv) and pyrrolidine $(57.5 \mu \mathrm{L}, \rho=0.866 \mathrm{~g} / \mathrm{mL}$, $700 \mu \mathrm{mol}, 3.50$ equiv) following General Procedure $\mathrm{C}$, on $1.00 \mathrm{mmol}$ scale. The crude residue was purified via column chromatography on silica gel using 91:9 hexanes:ethyl acetate as the eluent to afford of the title compound as a yellow solid (223 mg, 88\%, $176 \mu \mathrm{mol})$.

$\mathbf{R}_{\mathbf{f}}(83: 17$ hexanes:ethyl acetate $)=0.35$.

m.p. $90-92{ }^{\circ} \mathrm{C}\left(\mathrm{CDCl}_{3}\right)$. 
${ }^{1} \mathbf{H}$ NMR $\left(600 \mathrm{MHz}, \mathrm{CDCl}_{3}\right) \delta 7.35(\mathrm{~d}, J=12.2 \mathrm{~Hz}, 1 \mathrm{H}), 5.59(\mathrm{~d}, J=12.2 \mathrm{~Hz}, 1 \mathrm{H}), 3.45-3.37(\mathrm{~m}, 4 \mathrm{H})$, 2.06-2.02 (m, $4 \mathrm{H})$.

${ }^{13}$ C NMR $\left(151 \mathrm{MHz}, \mathrm{CDCl}_{3}\right) \delta 146.0,126.6,116.2,112.9,105.1,77.6,49.9,25.2$.

IR (ATR) 3046, 2975, 2866, 2236, 2191, 1583, $1553 \mathrm{~cm}^{-1}$.

HRMS (APCI) m/z: [M+H] $]^{+}$Calcd for $\mathrm{C}_{10} \mathrm{H}_{10} \mathrm{BrN}_{3} 252.0136$; Found 252.0130.

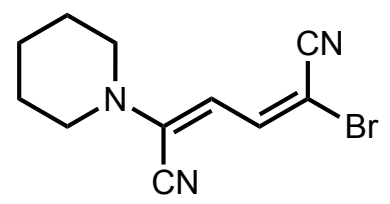

(2E,4E)-2-Bromo-5-(piperidin-1-yl)hexa-2,4-dienedinitrile (8b): The title compound was prepared from bis(2-bromoacrylonitrile) (3b) and piperidine $(69.1 \mu \mathrm{L}, \rho=0.862 \mathrm{~g} / \mathrm{mL}, 700 \mu \mathrm{mol}, 3.50$ equiv) following General Procedure C. The crude residue was purified via column chromatography on silica gel using 91:9 hexanes:ethyl acetate as the eluent to afford the title compound as a yellow solid (49.5 mg, 93\%, $186 \mu \mathrm{mol})$.

$\mathbf{R}_{\mathbf{f}}(83: 17$ hexanes:ethyl acetate $)=0.44$.

m.p. $83-85^{\circ} \mathrm{C}\left(\mathrm{CDCl}_{3}\right)$.

${ }^{1}$ H NMR $\left(600 \mathrm{MHz}, \mathrm{CDCl}_{3}\right) \delta 7.43(\mathrm{~d}, J=12.1 \mathrm{~Hz}, 1 \mathrm{H}), 5.87(\mathrm{~d}, J=12.1 \mathrm{~Hz}, 1 \mathrm{H}), 3.36-3.32(\mathrm{~m}, 4 \mathrm{H})$, $1.80(\mathrm{~m}, 4 \mathrm{H}), 1.68-1.65(\mathrm{~m}, 4 \mathrm{H})$.

${ }^{13}$ C NMR $\left(151 \mathrm{MHz}, \mathrm{CDCl}_{3}\right) \delta 146.3,129.7,115.8,112.8,106.9,79.8,49.7,25.1,23.8$.

IR (ATR) 2939, 2855, 2226, 2198, 1568, $1550 \mathrm{~cm}^{-1}$.

HRMS (APCI) m/z: $[\mathrm{M}+\mathrm{H}]^{+}$Calcd for $\mathrm{C}_{11} \mathrm{H}_{12} \mathrm{BrN}_{3} 266.0293$; Found 266.0285.

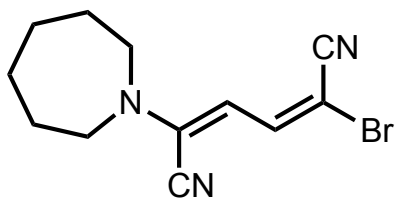

(2E,4E)-2-(Azepan-1-yl)-5-bromohexa-2,4-dienedinitrile (9b): The title compound was prepared from bis(2-bromoacrylonitrile) (3b) and azepane $(78.9 \mu \mathrm{L}, \rho=0.880 \mathrm{~g} / \mathrm{mL}, 700 \mu \mathrm{mol}, 3.50$ equiv) following General Procedure C. The crude residue was purified via column chromatography on silica gel using 12:1 hexanes:ethyl acetate as the eluent to afford the title compound as a yellow oil that solidified on standing (51.4 mg, 92\%, $184 \mu \mathrm{mol})$.

$\mathbf{R}_{\mathbf{f}}(83: 17$ hexanes:ethyl acetate $)=0.42$. 
m.p. $52-54{ }^{\circ} \mathrm{C}\left(\mathrm{CDCl}_{3}\right)$.

${ }^{1} \mathbf{H}$ NMR $\left(600 \mathrm{MHz}, \mathrm{CDCl}_{3}\right) \delta 7.41(\mathrm{~d}, J=12.1 \mathrm{~Hz}, 1 \mathrm{H}), 5.72(\mathrm{~d}, J=12.1 \mathrm{~Hz}, 1 \mathrm{H}), 3.49-3.45(\mathrm{~m}, 4 \mathrm{H})$, $1.82-1.77$ (m, 4 H), 1.62-1.57 (m, 4H).

${ }^{13}$ C NMR (151 MHz, $\left.\mathrm{CDCl}_{3}\right) \delta 146.4,129.1,116.2,112.7,104.5,77.9,51.9,27.8,27.1$.

IR (ATR) 2928, 2856, 2227, 2197, $1567 \mathrm{~cm}^{-1}$.

HRMS (APCI) m/z: [M+H] $]^{+}$Calcd for $\mathrm{C}_{12} \mathrm{H}_{14} \mathrm{BrN}_{3} 280.0449$; Found 280.0443.

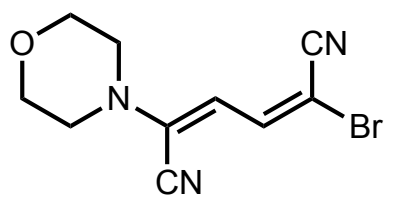

(2E,4E)-2-Bromo-5-morpholinohexa-2,4-dienedinitrile (10b): The title compound was prepared from bis(2-bromoacrylonitrile) (3b) and morpholine $(60.4 \mu \mathrm{L}, \rho=1.01 \mathrm{~g} / \mathrm{mL}, 700 \mu \mathrm{mol}, 3.50$ equiv) following General Procedure C. The crude residue was purified via column chromatography on silica gel using 83:17 hexanes:ethyl acetate as the eluent to afford the title compound as a yellow solid (53.2 $\mathrm{mg}, 99 \%, 198 \mu \mathrm{mol})$.

$\mathbf{R}_{\mathbf{f}}(83: 17$ hexanes:ethyl acetate $)=0.23$.

m.p. ${ }^{124}-126^{\circ} \mathrm{C}\left(\mathrm{CDCl}_{3}\right)$.

${ }^{1} \mathbf{H}$ NMR $\left(600 \mathrm{MHz}, \mathrm{CDCl}_{3}\right) \delta 7.44(\mathrm{~d}, 12.0 \mathrm{~Hz}, 1 \mathrm{H}), 5.93(\mathrm{~d}, 12.0 \mathrm{~Hz}, 1 \mathrm{H}), 3.81-3.76(\mathrm{~m}, 4 \mathrm{H}), 3.33-3.29$ $(\mathrm{m}, 4 \mathrm{H})$.

${ }^{13}$ C NMR $\left(151 \mathrm{MHz}, \mathrm{CDCl}_{3}\right) \delta 145.4,129.7,115.4,112.4,108.6,82.4,65.8,48.2$.

IR (ATR) 2967, 2922, 2881, 2854, 2223, 2205, 1742, 1584, $1548 \mathrm{~cm}^{-1}$.

HRMS (APCI) m/z: [M+H] $]^{+}$Calcd for $\mathrm{C}_{10} \mathrm{H}_{11} \mathrm{BrN}_{3} \mathrm{O}$ 268.0085; Found 268.0079.<smiles>N#C/C(Br)=C\C=C(/Br)N1CCSCC1</smiles>

(2E,4E)-2-Bromo-5-thiomorpholinohexa-2,4-dienedinitrile (11b): The title compound was prepared from bis(2-bromoacrylonitrile) (3b) and thiomorpholine $(70.1 \mu \mathrm{L}, \rho=1.03 \mathrm{~g} / \mathrm{mL}, 700 \mu \mathrm{mol}, 3.50$ equiv) following General Procedure C. The crude residue was purified via column chromatography on silica gel using 83:17 hexanes:ethyl acetate as the eluent to afford of the title compound as a yellow oil (55.3 $\mathrm{mg}$, 97\%, $194 \mu \mathrm{mol})$. 
$\mathbf{R}_{\mathbf{f}}(83: 17$ hexanes: ethyl acetate $)=0.41$.

${ }^{1}$ H NMR $\left(600 \mathrm{MHz}, \mathrm{CDCl}_{3}\right) \delta 7.43(\mathrm{~d}, J=12.0 \mathrm{~Hz}, 1 \mathrm{H}), 5.90(\mathrm{~d}, J=12.0 \mathrm{~Hz}, 1 \mathrm{H}), 3.74-3.70(\mathrm{~m}, 4 \mathrm{H})$, $2.72-2.69(\mathrm{~m}, 4 \mathrm{H})$.

${ }^{13}$ C NMR $\left(151 \mathrm{MHz}, \mathrm{CDCl}_{3}\right) \delta 145.6,128.3,115.4,112.3,108.1,81.4,51.2,26.3$.

IR (ATR) 3065, 2933, 2805, 2222, 2206, 1580, $1552 \mathrm{~cm}^{-1}$.

HRMS (APCI) m/z: [M+H] $]^{+}$Calcd for $\mathrm{C}_{10} \mathrm{H}_{10} \mathrm{BrN}_{3} \mathrm{~S} 283.9857$; Found 283.9854 .

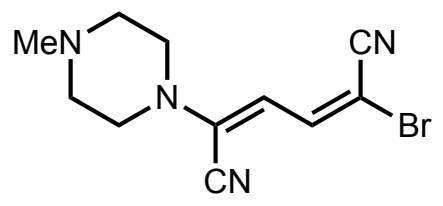

(2E,4E)-2-Bromo-5-(4-methylpiperazin-1-yl)hexa-2,4-dienedinitrile (12b): The title compound was prepared from bis(2-bromoacrylonitrile) (3b) and 1-methylpiperazine $(77.7 \mu \mathrm{L}, \rho=0.903 \mathrm{~g} / \mathrm{mL}, 700 \mu \mathrm{mol}$, 3.50 equiv) following General Procedure C. The crude residue was purified via column chromatography on silica gel using 99:1 dichloromethane:methanol as the eluent to afford the title compound as a yellow solid (52.2 mg, 93\%, $186 \mu \mathrm{mol})$.

$\mathbf{R}_{\mathbf{f}}(50: 50$ hexanes:ethyl acetate $)=0.12$.

m.p. $137-139^{\circ} \mathrm{C}\left(\mathrm{CDCl}_{3}\right)$.

${ }^{1}$ H NMR $\left(600 \mathrm{MHz}, \mathrm{CDCl}_{3}\right) \delta 7.42(\mathrm{~d}, J=12.0 \mathrm{~Hz}, 1 \mathrm{H}), 5.89(\mathrm{~d}, J=12.0 \mathrm{~Hz}, 1 \mathrm{H}), 3.37-3.32(\mathrm{~m}, 4 \mathrm{H})$, 2.52-2.47 (m, 4H), $2.33(\mathrm{~s}, 3 \mathrm{H})$.

${ }^{13}$ C NMR $\left(151 \mathrm{MHz}, \mathrm{CDCl}_{3}\right) \delta 145.8,129.5,115.5,112.6,108.0,81.3,53.9,48.3,46.0$.

IR (ATR) 2940, 2848, 2799, 2229, 2201, $1573 \mathrm{~cm}^{-1}$.

HRMS (APCI) m/z: [M+H] $]^{+}$Calcd for $\mathrm{C}_{11} \mathrm{H}_{13} \mathrm{BrN}_{4}$ 281.0402; Found 281.0398.<smiles>N#C/C(Br)=C\C=C(/C#N)N1CCC(=O)CC1</smiles>

(2E,4E)-2-Bromo-5-(4-oxopiperidin-1-yl)hexa-2,4-dienedinitrile (13b): The title compound was prepared from bis(2-bromoacrylonitrile) (3b) and 4-piperidone hydrochloride (94.9 mg, $700 \mu \mathrm{mol}, 3.50$ equiv) following General Procedure D. The crude residue was purified via column chromatography on silica gel using 75:25 hexanes:ethyl acetate as the eluent to afford the title compound as a yellow solid (48.6 $\mathrm{mg}, 87 \%, 174 \mu \mathrm{mol})$. 
$\mathbf{R}_{\mathbf{f}}(75: 25$ hexanes:ethyl acetate $)=0.31$.

m.p. $96-98^{\circ} \mathrm{C}\left(\mathrm{CDCl}_{3}\right)$.

${ }^{1}$ H NMR $\left(600 \mathrm{MHz}, \mathrm{CDCl}_{3}\right) 7.45(\mathrm{~d}, J=11.9 \mathrm{~Hz}, 1 \mathrm{H}), 5.95(\mathrm{~d}, J=11.9 \mathrm{~Hz}, 1 \mathrm{H}), 3.73(\mathrm{t}, J=6.3 \mathrm{~Hz}, 4 \mathrm{H})$, $2.62(\mathrm{t}, J=6.3 \mathrm{~Hz}, 4 \mathrm{H})$.

${ }^{13}$ C NMR $\left(151 \mathrm{MHz}, \mathrm{CDCl}_{3}\right) \delta 204.4,145.3,128.0,115.2,112.3,108.5,82.4,46.9,39.7$.

IR (ATR) 2958, 2922, 2853, 2228, 2200, 1719, 1572, $1554 \mathrm{~cm}^{-1}$.

HRMS (APCI) m/z: [M+H] $]^{+}$Calcd for $\mathrm{C}_{11} \mathrm{H}_{10} \mathrm{BrN}_{3} \mathrm{O}$ 280.0085; Found 280.0080.

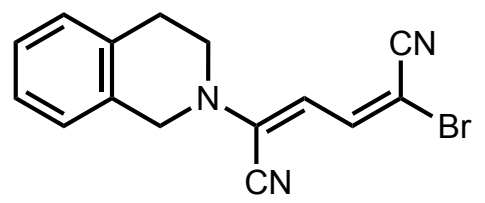

(2E,4E)-2-bromo-5-(3,4-dihydroisoquinolin-2(1H)-yl)hexa-2,4-dienedinitrile $\quad$ (14b): The title compound was prepared from bis(2-bromoacrylonitrile) (3b) and 1,2,3,4-tetrahydroisoquinoline $(88.8 \mu \mathrm{L}$, $\rho=1.05 \mathrm{~g} / \mathrm{mL}, 700 \mu \mathrm{mol}, 3.50$ equiv) following General Procedure C. The crude residue was purified via column chromatography on silica gel using 50:50 hexanes:dichloromethane as the eluent to afford the title compound as a yellow solid $(58.3 \mathrm{mg}, 93 \%, 186 \mu \mathrm{mol})$.

$\mathbf{R}_{\mathbf{f}}(83: 17$ hexanes:ethyl acetate $)=0.53$.

m.p. $91{ }^{\circ} \mathrm{C}$ (decomp.) $\left(\mathrm{CDCl}_{3}\right)$.

${ }^{1}$ H NMR $\left(600 \mathrm{MHz}, \mathrm{CDCl}_{3}\right) \delta 7.47(\mathrm{~d}, J=12.0 \mathrm{~Hz}, 1 \mathrm{H}), 7.29-7.17(\mathrm{~m}, 4 \mathrm{H}), 5.90(\mathrm{~d}, J=12 \mathrm{~Hz}, 1 \mathrm{H}), 4.46$ (s, 2H), $3.71(\mathrm{t}, J=5.9 \mathrm{~Hz}, 2 \mathrm{H}), 2.99(\mathrm{t}, J=5.9 \mathrm{~Hz}, 2 \mathrm{H})$.

${ }^{13}$ C NMR $\left(151 \mathrm{MHz}, \mathrm{CDCl}_{3}\right) \delta 145.9,133.6,130.8,128.7,128.5,127.5,127.0,126.3,115.7,112.5,106.3$, $80.0,49.6,46.9,28.6$.

IR (ATR) 3062, 3027, 2921, 2852, 2228, 2200, 1735, 1569, $1552 \mathrm{~cm}^{-1}$.

HRMS (APCI) m/z: [M+H] $]^{+}$Calcd for $\mathrm{C}_{15} \mathrm{H}_{13} \mathrm{BrN}_{3}$ 314.0293; Found 314.0290.

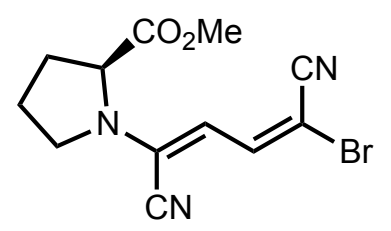

((1E,3E)-Methyl-4-bromo-1,4-dicyanobuta-1,3-dien-1-yl)-L-prolinate (15b): The title compound was prepared from bis(2-bromoacrylonitrile) (3b) and $L$-proline methyl ester hydrochloride (116 mg, $700 \mu \mathrm{mol}$, 
3.50 equiv) following General Procedure D. The crude residue was purified via column chromatography on silica gel using 80:20 hexanes:ethyl acetate as the eluent to afford the title compound as a yellow/orange oil (24.7 mg, 40\%, $80 \mu \mathrm{mol})$.

$\mathbf{R}_{\mathbf{f}}(50: 50$ hexanes: ethyl acetate $)=0.60$.

${ }^{1}$ H NMR $\left(600 \mathrm{MHz}, \mathrm{CDCl}_{3}\right) \delta 7.37(\mathrm{~d}, J=12.1 \mathrm{~Hz}, 1 \mathrm{H}), 5.70(\mathrm{~d}, J=12.1 \mathrm{~Hz}, 1 \mathrm{H}), 4.41(\mathrm{dd}, J=8.6,2.7$ $\mathrm{Hz}, 1 \mathrm{H}), 3.79$ (s, 3H), 3.55-3.50 (m, 1H), 3.42-3.36 (m, 1H), 2.36-2.28 (m, 1H), 2.26-2.20 (m, 1H), 2.12$2.04(\mathrm{~m}, 2 \mathrm{H})$.

${ }^{13}$ C NMR (151 MHz, $\left.\mathrm{CDCl}_{3}\right) \delta 171.6,145.6,126.0,115.6,112.7,107.3,80.2,62.7,53.0,49.7,30.4,23.6$. IR (ATR) 2954, 2232, 2200, 1742, $1577 \mathrm{~cm}^{-1}$.

HRMS (APCI) m/z: [M+H] $]^{+}$Calcd for $\mathrm{C}_{12} \mathrm{H}_{12} \mathrm{BrN}_{3} \mathrm{O}_{2} 310.0191$; Found 310.0187.<smiles>CN(Cc1ccccc1)/C(C#N)=C/C=C(/Br)C#N</smiles>

(2E,4E)-2-(Benzyl(methyl)amino)-5-bromohexa-2,4-dienedinitrile (16b): The title compound was prepared from bis(2-bromoacrylonitrile) (3b) and $N$-methylbenzylamine $(90.3 \mu \mathrm{L}, \rho=0.939 \mathrm{~g} / \mathrm{mL}, 700$ $\mu$ mol, 3.50 equiv) following General Procedure C. The crude residue was purified via column chromatography on silica gel using 91:9 hexanes:ethyl acetate as the eluent to afford of the title compound as a yellow/orange oil $(51.1 \mathrm{mg}, 85 \%, 170 \mu \mathrm{mol})$.

$\mathbf{R}_{\mathbf{f}}(83: 17$ hexanes:ethyl acetate $)=0.45$.

${ }^{1} \mathbf{H}$ NMR $\left(600 \mathrm{MHz}, \mathrm{CDCl}_{3}\right) \delta 7.45(\mathrm{~d}, J=12.1 \mathrm{~Hz}, 1 \mathrm{H}), 7.41-7.37(\mathrm{~m}, 2 \mathrm{H}), 7.36-7.33(\mathrm{~m}, 1 \mathrm{H}), 7.22-7.19$ $(\mathrm{m}, 2 \mathrm{H}), 5.79(\mathrm{~d}, J=12.1 \mathrm{~Hz}, 1 \mathrm{H}), 4.60(\mathrm{~s}, 2 \mathrm{H}), 2.90(\mathrm{~s}, 3 \mathrm{H})$.

${ }^{13}$ C NMR (151 MHz, $\left.\mathrm{CDCl}_{3}\right) \delta 146.1,135.1,129.3,129.3,128.6,127.5,115.8,112.8,106.6,79.9,58.3$, 37.9.

IR (ATR) 3029, 2926, 2230, 2199, $1572 \mathrm{~cm}^{-1}$.

HRMS (APCI) m/z: [M+H] $]^{+}$Calcd for $\mathrm{C}_{14} \mathrm{H}_{13} \mathrm{BrN}_{3}$ 302.0293; Found 302.0291.<smiles>CN(CCc1ccccc1)/C(C#N)=C/C=C(\Br)C#N</smiles> 
(2E,4E)-2-Bromo-5-(methyl(phenethyl)amino)hexa-2,4-dienedinitrile (17b): The title compound was prepared from bis(2-bromoacrylonitrile) (3b) and $N$-methylphenethylamine (102 $\mu \mathrm{L}, \rho=0.930 \mathrm{~g} / \mathrm{mL}, 700$ $\mu$ mol, 3.50 equiv) following General Procedure C. The crude residue was purified via column chromatography on silica gel using 91:9 hexanes:ethyl acetate as the eluent to afford of the title compound as a yellow solid $(53.8 \mathrm{mg}, 85 \%, 170 \mu \mathrm{mol})$.

$\mathbf{R}_{\mathbf{f}}(83: 17$ hexanes: ethyl acetate $)=0.35$.

m.p. $76-78{ }^{\circ} \mathrm{C}\left(\mathrm{CDCl}_{3}\right)$.

${ }^{1} \mathbf{H}$ NMR $\left(600 \mathrm{MHz}, \mathrm{CDCl}_{3}\right) \delta 7.35(\mathrm{~d}, J=12.1 \mathrm{~Hz}, 1 \mathrm{H}), 7.34-7.31(\mathrm{~m}, 2 \mathrm{H}), 7.29-7.25(\mathrm{~m}, 1 \mathrm{H}), 7.19-7.16$ (m, 2H), $5.66(\mathrm{~d}, J=12.1 \mathrm{~Hz}, 1 \mathrm{H}), 3.66(\mathrm{t}, J=7.3 \mathrm{~Hz}, 2 \mathrm{H}), 2.89$ (t, $J=7.3 \mathrm{~Hz}, 2 \mathrm{H}), 2.88(\mathrm{~s}, 3 \mathrm{H})$.

${ }^{13}$ C NMR $\left(151 \mathrm{MHz}, \mathrm{CDCl}_{3}\right) \delta 146.1,137.2,129.0,129.0,128.9,127.2,115.9,112.5,105.8,79.2,56.7$, 38.6, 34.8.

IR (ATR) 3026, 2931, 2230, 2198, $1575 \mathrm{~cm}^{-1}$.

HRMS (APCI) m/z: [M+H] $]^{+}$Calcd for $\mathrm{C}_{15} \mathrm{H}_{15} \mathrm{BrN}_{3}$ 316.0449; Found 316.0447.<smiles>CN(C)/C(C#N)=C/C=C(\Br)C#N</smiles>

(2E,4E)-2-Bromo-5-(dimethylamino)hexa-2,4-dienedinitrile (18b): The title compound was prepared from bis(2-bromoacrylonitrile) (3b) and dimethylamine hydrochloride (57.1 $\mathrm{mg}, 700 \mu \mathrm{mol}, 3.50$ equiv) following General Procedure D. The crude residue was purified via column chromatography on silica gel using 83:17 hexanes:ethyl acetate as the eluent to afford of the title compound as a yellow solid (40.4 mg, $89 \%, 178 \mu \mathrm{mol})$.

$\mathbf{R}_{\mathbf{f}}(83: 17$ hexanes:ethyl acetate $)=0.22$.

m.p. $116-118{ }^{\circ} \mathrm{C}\left(\mathrm{CDCl}_{3}\right)$.

${ }^{1} \mathbf{H}$ NMR $\left(600 \mathrm{MHz}, \mathrm{CDCl}_{3}\right) \delta 7.38(\mathrm{~d}, \mathrm{~J}=12.1 \mathrm{~Hz}, 1 \mathrm{H}), 5.69(\mathrm{~d}, \mathrm{~J}=12.1 \mathrm{~Hz}, 1 \mathrm{H}), 3.05(\mathrm{~s}, 3 \mathrm{H})$.

${ }^{13}$ C NMR $\left(151 \mathrm{MHz}, \mathrm{CDCl}_{3}\right) \delta 146.1,129.8,115.9,112.6,105.9,79.2,41.0$.

IR (ATR) 3068, 3021, 2923, 2856, 2229, 2200, $1584 \mathrm{~cm}^{-1}$.

HRMS (APCI) m/z: [M+H] $]^{+}$Calcd for $\mathrm{C}_{8} \mathrm{H}_{8} \mathrm{BrN}_{3}$ 225.9980; Found 225.9977. 


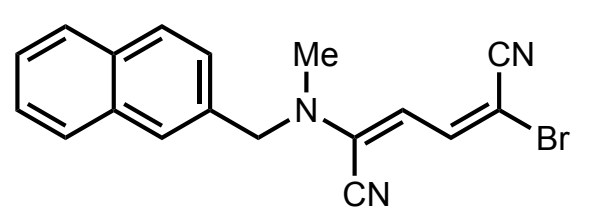

(2E,4E)-2-Bromo-5-(methyl(naphthalen-2-ylmethyl)amino)hexa-2,4-dienedinitrile (19b): The title compound was prepared from bis(2-bromoacrylonitrile) (3b) and $N$-methyl-1-(naphthalen-2yl)methanamine (145 mg, $700 \mu \mathrm{mol}, 3.50$ equiv) following General Procedure D. The crude residue was purified via column chromatography on silica gel using 91:9 hexanes:ethyl acetate as the eluent to afford the title compound as a yellow/orange crystalline solid $(62.0 \mathrm{mg}, 88 \%, 176 \mu \mathrm{mol})$.

$\mathbf{R}_{\mathbf{f}}(83: 17$ hexanes: ethyl acetate $)=0.39$.

m.p. $92-94{ }^{\circ} \mathrm{C}\left(\mathrm{CDCl}_{3}\right)$.

${ }^{1}$ H NMR (600 MHz, $\left.\mathrm{CDCl}_{3}\right) \delta$ 7.90-7.82 (m, 3H), $7.65(\mathrm{~s}, 1 \mathrm{H}), 7.55-7.50(\mathrm{~m}, 2 \mathrm{H}), 7.47(\mathrm{~d}, J=12.1 \mathrm{~Hz}$, 1H), 7.30 (dd, $J=8.4,1.9 \mathrm{~Hz}, 1 \mathrm{H}), 5.84$ (d, $J=12.1 \mathrm{~Hz}, 1 \mathrm{H}), 4.75$ (s, 2H), 2.94 (s, 3H).

${ }^{13}$ C NMR (151 MHz, $\left.\mathrm{CDCl}_{3}\right) \delta 146.0,133.3,133.2,132.5,129.3,127.94,127.89,126.8,126.71,126.65$, $124.9,115.7,112.9,106.6,80.0,58.4,37.9$.

IR (ATR) 3055, 2924, 2853, 2229, 2200, 1741, $1574 \mathrm{~cm}^{-1}$.

HRMS (APCI) m/z: [M+H] $]^{+}$Calcd for $\mathrm{C}_{18} \mathrm{H}_{15} \mathrm{BrN}_{3}$ 352.0449; Found 352.0445.

\section{Preparation and Characterization of Malonate Additions Products}

General procedure for the addition of malonate nucleophiles to bis(2-haloacrylonitrile) - General Procedure E.

To a $4 \mathrm{~mL}$ dram vial equipped with magnetic Teflon ${ }^{\circledR}$-coated stir bar under $\mathrm{N}_{2}$ was added the appropriate malonate derivative $(600 \mu \mathrm{mol}, 3.00$ equiv) followed by $0.50 \mathrm{~mL}$ of anhydrous THF. NaH $(24.0 \mathrm{mg}, 60 \%$ mineral oil dispersion, $600 \mu \mathrm{mol}, 3.00$ equiv) was then added and the reaction mixture was stirred until effervescence halted. To the stirred solution was added a solution of bis(2-bromoacrylonitrile) (3b) (52.4 $\mathrm{mg}, 200 \mu \mathrm{mol}, 1.00$ equiv) in $1.50 \mathrm{~mL}$ of anhydrous THF $(0.2 \mathrm{M})$ and the reactions were stirred at $23^{\circ} \mathrm{C}$ until the conclusion of the reaction as determined by TLC. The solvent was then concentrated in vacuo and the products by silica gel flash column chromatography. 


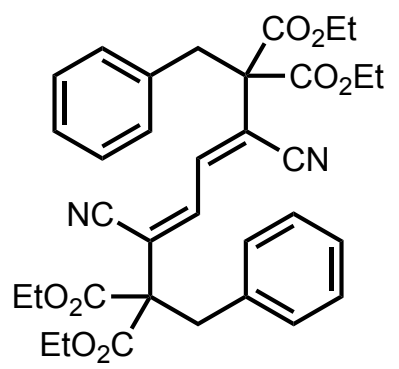

(3Z,5Z)-Tetraethyl-3,6-dicyano-1,8-diphenylocta-3,5-diene-2,2,7,7-tetracarboxylate (20a): The title compound was prepared from diethyl 2-benzylmalonate $(142 \mu \mathrm{L}, \rho=1.06 \mathrm{~g} / \mathrm{mL}, 600 \mu \mathrm{mol}, 3.00$ equiv) following General Procedure E. The crude residue was purified via column chromatography on silica gel using 91:9 hexanes:ethyl acetate as the eluent to afford the title compound as a colorless solid (101 mg, $84 \%, 168 \mu \mathrm{mol})$.

$\mathbf{R}_{\mathbf{f}}(83: 17$ hexanes:ethyl acetate $)=0.17$.

m.p. $76-78{ }^{\circ} \mathrm{C}\left(\mathrm{CDCl}_{3}\right)$.

${ }^{1}$ H NMR (600 MHz, $\left.\mathrm{CDCl}_{3}\right)$ 8 7.27-7.24 (m, 8H), 7.13-7.10 (m, 4H), 4.25 (q, J= 7.1 Hz, 8H), 3.54 (s, $4 \mathrm{H}), 1.26(\mathrm{t}, J=7.1 \mathrm{~Hz}, 12 \mathrm{H})$.

${ }^{13}$ C NMR (151 MHz, $\left.\mathrm{CDCl}_{3}\right) \delta 167.2,141.9,134.1,130.4,128.6,127.9,119.5,114.6,63.1,62.9,40.7$, 14.0.

IR (ATR) 2982, 2938, 2215, 1737, $1454 \mathrm{~cm}^{-1}$.

HRMS (APCI) m/z: [M+H] $]^{+}$Calcd for $\mathrm{C}_{34} \mathrm{H}_{37} \mathrm{~N}_{2} \mathrm{O}_{8}$ 601.2550; Found 601.2549.<smiles>CCOCC(C)(C(=O)OCC)/C(C)=C/C=C(\C#N)C(Cc1ccc(Br)cc1)(Cc1ccc(Br)cc1)C(=O)OCC</smiles>

\section{(3Z,5Z)-Tetraethyl-1,8-bis(4-bromophenyl)-3,6-dicyanoocta-3,5-diene-2,2,7,7-tetracarboxylate}

(20b): The title compound was prepared from diethyl 2-(4-bromobenzyl)malonate (198 mg, $600 \mu \mathrm{mol}, 3.00$ equiv) following General Procedure E. The crude residue was purified via column chromatography on silica gel using 91:9 hexanes:ethyl acetate as the eluent to afford of the title compound as a colorless crystalline solid (130 mg, 86\%, $172 \mu \mathrm{mol}$ ).

$\mathbf{R}_{\mathbf{f}}(83: 17$ hexanes: ethyl acetate $)=0.26$. 
m.p. $88-90{ }^{\circ} \mathrm{C}\left(\mathrm{CDCl}_{3}\right)$.

${ }^{1}$ H NMR (600 MHz, $\left.\mathrm{CDCl}_{3}\right) 7.40(\mathrm{~d}, J=7.4 \mathrm{~Hz}, 4 \mathrm{H}), 7.30$ (s, $\left.2 \mathrm{H}\right), 7.03(\mathrm{~d}, J=7.4 \mathrm{~Hz}, 4 \mathrm{H}), 4.32-4.22$ (m, $8 \mathrm{H}), 3.49$ (s, $4 \mathrm{H}), 1.27$ (t, $J=7.2 \mathrm{~Hz}, 12 \mathrm{H})$.

${ }^{13}$ C NMR (151 MHz, $\left.\mathrm{CDCl}_{3}\right) \delta 167.0,141.6,133.1,132.2,131.7,122.1,119.8,114.6,63.3,62.7,40.0$, 14.0 .

IR (ATR) 2982, 2938, 2216, 1736, $1487 \mathrm{~cm}^{-1}$.

HRMS (APCI) m/z: [M+H] $]^{+}$Calcd for $\mathrm{C}_{34} \mathrm{H}_{34} \mathrm{Br}_{2} \mathrm{~N}_{2} \mathrm{O}_{8}$ 757.0760; Found 757.0758.

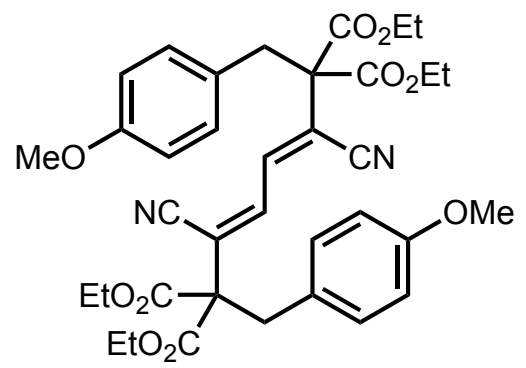

\section{(3Z,5Z)-Tetraethyl-3,6-dicyano-1,8-bis(4-methoxyphenyl)octa-3,5-diene-2,2,7,7-tetracarboxylate}

(20c): The title compound was prepared from diethyl 2-(4-methoxybenzyl)malonate (168 mg, $600 \mu \mathrm{mol}$, 3.00 equiv) following General Procedure E. The crude residue was purified via column chromatography on silica gel using 83:17 hexanes:ethyl acetate as the eluent to afford the title compound as a colorless oil (123 mg, 93\%, $186 \mu \mathrm{mol})$.

$\mathbf{R}_{\mathbf{f}}(83: 17$ hexanes: ethyl acetate $)=0.09$.

${ }^{1} \mathrm{H}$ NMR $\left(600 \mathrm{MHz}, \mathrm{CDCl}_{3}\right) \delta 7.26(\mathrm{~s}, 2 \mathrm{H}), 7.04(\mathrm{~d}, J=8.7 \mathrm{~Hz}, 4 \mathrm{H}), 6.80(\mathrm{~d}, J=8.7 \mathrm{~Hz}, 4 \mathrm{H}), 4.27(\mathrm{q}, J=$ $7.2 \mathrm{~Hz}, 8 \mathrm{H}), 3.78$ (s, 6H), 1.49 (s, 4H), 1.27 (t, $J=7.2 \mathrm{~Hz}, 12 \mathrm{H})$.

${ }^{13}$ C NMR (151 MHz, $\left.\mathrm{CDCl}_{3}\right) \delta 167.2,159.2,141.9,131.5,125.9,119.5,114.7,114.0,63.0$ (two peaks), $55.3,40.0,14.0$.

IR (ATR) 2982, 2937, 2837, 2215, 1737, 1611, $1513 \mathrm{~cm}^{-1}$.

HRMS (APCI) m/z: [M+H] $]^{+}$Calcd for $\mathrm{C}_{36} \mathrm{H}_{41} \mathrm{~N}_{2} \mathrm{O}_{10}$ 661.2761; Found 661.2764. 


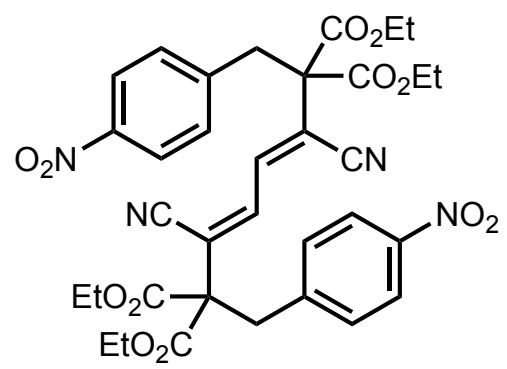

(3Z,5Z)-Tetraethyl-3,6-dicyano-1,8-bis(4-nitrophenyl)octa-3,5-diene-2,2,7,7-tetracarboxylate (20d): The title compound was prepared from diethyl 2-(4-nitrobenzyl)malonate (177 mg, $600 \mu \mathrm{mol}, 3.00$ equiv) following General Procedure E. The crude residue was purified via column chromatography on silica gel using 80:20 hexanes:ethyl acetate as the eluent to afford of the title compound as a pale yellow crystalline solid (105 mg, 76\%, $152 \mu \mathrm{mol})$.

$\mathbf{R}_{\mathbf{f}}(83: 17$ hexanes: ethyl acetate $)=0.09$.

m.p. $118-120{ }^{\circ} \mathrm{C}\left(\mathrm{CDCl}_{3}\right)$.

${ }^{1}$ H NMR (600 MHz, $\left.\mathrm{CDCl}_{3}\right) 8.14(\mathrm{~d}, J=8.7 \mathrm{~Hz}, 4 \mathrm{H}), 7.37$ (d, $\left.J=8.7 \mathrm{~Hz}, 4 \mathrm{H}\right), 7.28(\mathrm{~s}, 2 \mathrm{H}), 4.33-4.23$ $(\mathrm{m}, 8 \mathrm{H}), 3.62(\mathrm{~s}, 4 \mathrm{H}), 1.26(\mathrm{t}, J=7.3 \mathrm{~Hz}, 12 \mathrm{H})$.

${ }^{13}$ C NMR $\delta 166.6,147.6,141.8,141.4,131.5,123.7,119.9,114.4,63.6,62.5,40.2,13.9$.

IR (ATR) 2984, 2217, 1734, 1606, $1520 \mathrm{~cm}^{-1}$.

HRMS (APCI) m/z: [M+H] $]^{+}$Calcd for $\mathrm{C}_{34} \mathrm{H}_{35} \mathrm{~N}_{4} \mathrm{O}_{12}$ 691.2251; Found 691.2250.<smiles>CCOC(=O)C(Cc1ccc(C)cc1)(Cc1ccc(C)cc1)/C(C#N)=C/C=C(\C)C(C(=O)OCC)(C(=O)OCC)C(=O)OCC</smiles>

(3Z,5Z)-Tetraethyl-3,6-dicyano-1,8-di-p-tolylocta-3,5-diene-2,2,7,7-tetracarboxylate (20e): The title compound was prepared from diethyl 2-(4-methylbenzyl)malonate (159 mg, $600 \mu \mathrm{mol}, 3.00$ equiv) following General Procedure E. The crude residue was purified via column chromatography on silica gel using 91:9 hexanes:ethyl acetate as the eluent to afford the title compound as a colorless oil (105 mg, 84\%, $168 \mu \mathrm{mol})$.

$\mathbf{R}_{\mathbf{f}}(83: 17$ hexanes:ethyl acetate $)=0.24$. 
${ }^{1}$ H NMR $\left(600 \mathrm{MHz}, \mathrm{CDCl}_{3}\right) \delta 7.25(\mathrm{~s}, 2 \mathrm{H}), 7.07$ (d, $\left.J=7.9 \mathrm{~Hz}, 4 \mathrm{H}\right), 7.00(\mathrm{~d}, J=8.1 \mathrm{~Hz}, 4 \mathrm{H}), 4.31-4.22$ (m, $8 \mathrm{H}), 3.51(\mathrm{~s}, 4 \mathrm{H}), 2.31(\mathrm{~s}, 6 \mathrm{H}), 1.28(\mathrm{t}, J=7.2 \mathrm{~Hz}, 12 \mathrm{H})$.

${ }^{13}$ C NMR (151 MHz, $\left.\mathrm{CDCl}_{3}\right) \delta 167.2,142.0,137.6,130.9,130.3,129.3,119.3,114.7,63.0,62.9,40.3$, 21.3, 14.0.

IR (ATR) 2982, 2937, 2214, 1737, 1515, $1445 \mathrm{~cm}^{-1}$.

HRMS (APCI) m/z: [M+H] $]^{+}$Calcd for $\mathrm{C}_{36} \mathrm{H}_{41} \mathrm{~N}_{2} \mathrm{O}_{8}$ 629.2863; Found 629.2865.

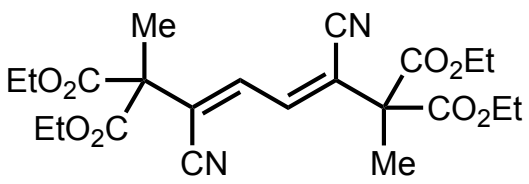

(3Z,5Z)-Tetraethyl-3,6-dicyanoocta-3,5-diene-2,2,7,7-tetracarboxylate (21): The title compound was prepared from diethyl 2-methylmalonate $(102 \mu \mathrm{L}, \rho=1.02 \mathrm{~g} / \mathrm{mL}, 600 \mu \mathrm{mol}, 3.00$ equiv $)$ following General Procedure E. The crude residue was purified via column chromatography on silica gel using 89:11 hexanes:ethyl acetate as the eluent to afford the title compound as a colorless oil ( $64.9 \mathrm{mg}, 72 \%, 144 \mu \mathrm{mol})$.

$\mathbf{R}_{\mathbf{f}}(83: 17$ hexanes:ethyl acetate $)=0.28$.

${ }^{1}$ H NMR $\left(600 \mathrm{MHz}, \mathrm{CDCl}_{3}\right) \delta 7.24(\mathrm{~s}, 2 \mathrm{H}), 4.29(\mathrm{q}, J=7.1 \mathrm{~Hz}, 8 \mathrm{H}), 1.77(\mathrm{~s}, 6 \mathrm{H}), 1.31(\mathrm{t}, J=7.1 \mathrm{~Hz}, 12 \mathrm{H})$.

${ }^{13}$ C NMR $\left(151 \mathrm{MHz}, \mathrm{CDCl}_{3}\right) \delta 168.2,139.1,121.5,114.8,63.2,57.8,20.6,14.0$.

IR (ATR) 2984, 2940, 2229, 1731, $1448 \mathrm{~cm}^{-1}$.

HRMS (APCI) m/z: [M+H] Calcd for $\mathrm{C}_{22} \mathrm{H}_{29} \mathrm{~N}_{2} \mathrm{O}_{8} 449.1924$; Found 449.1919.

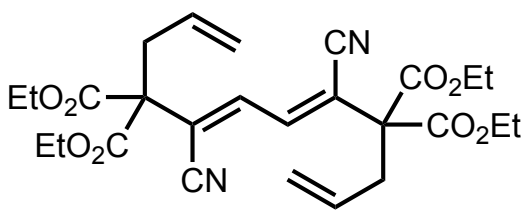

(5Z,7Z)-Tetraethyl-5,8-dicyanododeca-1,5,7,11-tetraene-4,4,9,9-tetracarboxylate (22): The title compound was prepared from diethyl 2-allylmalonate $(118 \mu \mathrm{L}, \rho=1.02 \mathrm{~g} / \mathrm{mL}, 600 \mu \mathrm{mol}, 3.00$ equiv) following General Procedure E. The crude residue was purified via column chromatography on silica gel using 91:9 hexanes:ethyl acetate as the eluent to afford the title compound as a colorless oil, $(74.9 \mathrm{mg}, 75 \%$, $150 \mu \mathrm{mol})$.

$\mathbf{R}_{\mathbf{f}}(83: 17$ hexanes: ethyl acetate $)=0.31$.

${ }^{1} \mathrm{H}$ NMR $\left(600 \mathrm{MHz}, \mathrm{CDCl}_{3}\right) \delta 7.41(\mathrm{~s}, 2 \mathrm{H}), 5.74(\mathrm{ddd}, J=17.0,10.1,7.5 \mathrm{~Hz}, 2 \mathrm{H}), 5.24(\mathrm{~d}, J=17.0 \mathrm{~Hz}$, 2H) $5.19(\mathrm{~d}, J=10.1 \mathrm{~Hz}, 2 \mathrm{H}), 4.29(\mathrm{q}, J=7.1 \mathrm{~Hz}, 8 \mathrm{H}), 2.97(\mathrm{~d}, J=7.5 \mathrm{~Hz}, 4 \mathrm{H}), 1.31(\mathrm{t}, J=7.1 \mathrm{~Hz}, 12 \mathrm{H})$. 
${ }^{13}$ C NMR $\left(151 \mathrm{MHz}, \mathrm{CDCl}_{3}\right) \delta 167.2,141.0,130.8,121.2,119.9,114.9,63.1,61.3,38.7,14.1$.

IR (ATR) 2983, 2938, 2217, 1733, $1445 \mathrm{~cm}^{-1}$.

HRMS (APCI) m/z: $[\mathrm{M}+\mathrm{H}]^{+}$Calcd for $\mathrm{C}_{26} \mathrm{H}_{33} \mathrm{~N}_{2} \mathrm{O}_{8}$ 501.2237; Found 501.2228.<smiles>CCOC(=O)C(C)(/C=C\C=C(/C)C(C/C=C\P)(C/C=C\c1ccccc1)C(=O)OCC)C(=O)OCC</smiles>

\section{(1E,5Z,7Z,11E)-Tetraethyl-5,8-dicyano-1,12-diphenyldodeca-1,5,7,11-tetraene-4,4,9,9-}

tetracarboxylate (23): The title compound was prepared from diethyl 2-cinnamylmalonate (166 mg, $600 \mu \mathrm{mol}, 3.00$ equiv) following General Procedure E. The crude residue was purified via column chromatography on silica gel using 91:9 hexanes:ethyl acetate as the eluent to afford of the title compound as a pale yellow oil $(127 \mathrm{mg}, 98 \%, 196 \mu \mathrm{mol})$.

$\mathbf{R}_{\mathbf{f}}(83: 17$ hexanes:ethyl acetate $)=0.21$.

${ }^{1}$ H NMR $\left(600 \mathrm{MHz}, \mathrm{CDCl}_{3}\right) \delta 7.44(\mathrm{~s}, 2 \mathrm{H}), 7.33-7.21(\mathrm{~m}, 10 \mathrm{H}), 6.56(\mathrm{~d}, J=15.8 \mathrm{~Hz}, 2 \mathrm{H}), 6.11(\mathrm{dd}, J=$ $15.8,7.3 \mathrm{~Hz}, 2 \mathrm{H}), 4.29(\mathrm{q}, J=7.1 \mathrm{~Hz}, 8 \mathrm{H}), 3.13(\mathrm{~d}, J=7.3 \mathrm{~Hz}, 4 \mathrm{H}), 1.28(\mathrm{t}, J=7.1 \mathrm{~Hz}, 12 \mathrm{H})$.

${ }^{13}$ C NMR $\left(151 \mathrm{MHz}, \mathrm{CDCl}_{3}\right) \delta 167.2,141.0,136.7,135.8,128.7,127.9,126.6,122.0,120.1,115.0,63.2$, $61.7,38.3,14.1$.

IR (ATR) 2981, 2937, 2220, 1733, $1447 \mathrm{~cm}^{-1}$.

HRMS (APCI) m/z: $[\mathrm{M}+\mathrm{H}]^{+}$Calcd for $\mathrm{C}_{38} \mathrm{H}_{41} \mathrm{~N}_{2} \mathrm{O}_{8}$ 653.2863; Found 653.2858.

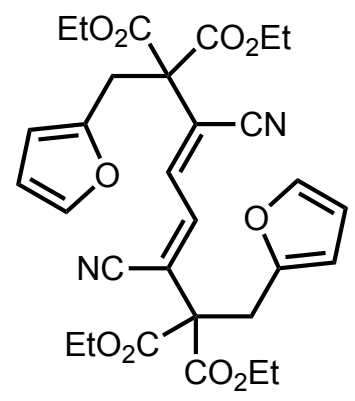

(3Z,5Z)-Tetraethyl-3,6-dicyano-1,8-di(furan-2-yl)octa-3,5-diene-2,2,7,7-tetracarboxylate (24) The title compound was prepared from diethyl 2-(furan-2-ylmethyl)malonate (144 mg, $600 \mu \mathrm{mol}, 3.00$ equiv) following General Procedure E. The crude residue was purified via column chromatography on silica gel using 83:17 hexanes:ethyl acetate as the eluent to afford the title compound as a colorless oil (86.4 mg, $74 \%, 148 \mu \mathrm{mol})$. 
$\mathbf{R}_{\mathbf{f}}(83: 17$ hexanes:ethyl acetate $)=0.10$.

${ }^{1}$ H NMR (600 MHz, $\left.\mathrm{CDCl}_{3}\right) \delta 7.31(\mathrm{~s}, 2 \mathrm{H}), 7.30(\mathrm{~d}, J=1.9 \mathrm{~Hz}, 2 \mathrm{H}), 6.28(\mathrm{dd}, J=3.0,1.9 \mathrm{~Hz}, 2 \mathrm{H}), 6.17$ $(\mathrm{d}, J=3.0 \mathrm{~Hz}, 2 \mathrm{H}), 4.31$ (q, $J=7.2 \mathrm{~Hz}, 8 \mathrm{H}), 3.60(\mathrm{~s}, 4 \mathrm{H}), 1.30(\mathrm{t}, J=7.2 \mathrm{~Hz}, 12 \mathrm{H})$.

${ }^{13}$ C NMR $\left(151 \mathrm{MHz}, \mathrm{CDCl}_{3}\right) \delta 166.9,148.2,142.6,141.5,119.2,114.4,110.7,110.2,63.3,61.7,33.3$, 14.0.

IR (ATR) 2984, 2939, 2217, 1736, 1504, $1445 \mathrm{~cm}^{-1}$.

HRMS (APCI) m/z: $[\mathrm{M}+\mathrm{H}]^{+}$Calcd for $\mathrm{C}_{30} \mathrm{H}_{33} \mathrm{~N}_{2} \mathrm{O}_{10}$ 581.2135; Found 581.2133.

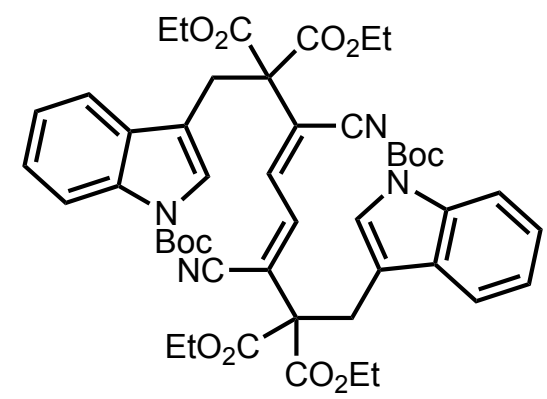

(3Z,5Z)-Tetraethyl-1,8-bis(1-(tert-butoxycarbonyl)-1H-indol-3-yl)-3,6-dicyanoocta-3,5-diene-2,2,7,7tetracarboxylate (25): The title compound was prepared from diethyl 2-((1-(tert-butoxycarbonyl)-1Hindol-3-yl)methyl)malonate (234 mg, $600 \mu \mathrm{mol}, 3.00$ equiv) following General Procedure E. The crude residue was purified via column chromatography on silica gel using 91:9 hexanes:ethyl acetate as the eluent to afford the title compound as a pale yellow powder (146 mg 83\%, $166 \mu \mathrm{mol})$.

$\mathbf{R}_{\mathbf{f}}(83: 17$ hexanes:ethyl acetate $)=0.17$.

m.p. $139-141^{\circ} \mathrm{C}\left(\mathrm{CDCl}_{3}\right)$.

${ }^{1}$ H NMR $\left(600 \mathrm{MHz}, \mathrm{CDCl}_{3}\right) \delta 8.09$ (s, 2H), 7.49 (s, 2H), 7.45 (d, J = 7.9 Hz, 2H), $7.36(\mathrm{~s}, 2 \mathrm{H}), 7.30-7.26$ (m, 2H), 7.21-7.18 (m, 2H), $4.20(\mathrm{q}, \mathrm{J}=7.1 \mathrm{~Hz}, 8 \mathrm{H}), 3.65-3.61(\mathrm{~m}, 4 \mathrm{H}), 1.21(\mathrm{t}, \mathrm{J}=7.1 \mathrm{~Hz}, 12 \mathrm{H})$.

${ }^{13}$ C NMR $\left(151 \mathrm{MHz}, \mathrm{CDCl}_{3}\right) \delta 167.2,149.6,141.5,130.8,125.7,124.6,122.6,120.1,118.8,115.4,114.7$, $113.1,84.0,63.2,62.2,30.2,28.3,13.9$.

IR (ATR) 2980, 2936, 2214, 1730, $1451 \mathrm{~cm}^{-1}$.

HRMS (APCI) m/z: $[\mathrm{M}+\mathrm{H}]^{+}$Calcd for $\mathrm{C}_{48} \mathrm{H}_{54} \mathrm{~N}_{4} \mathrm{O}_{12}$ 879.3816; Found 879.3817.

VII. Preparation and Characterization of Functionalization of $7 \mathrm{a}$ and $7 \mathrm{~b}$ Products 


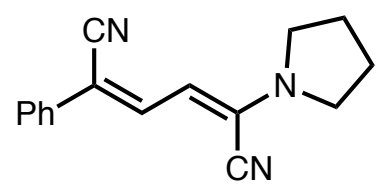

(2Z,4E)-2-Phenyl-5-(pyrrolidin-1-yl)hexa-2,4-dienedinitrile (28). (From Cl compound) A flame-dried $4 \mathrm{~mL}$ dram vial equipped with a septum cap and a magnetic Teflon ${ }^{\circledR}$-coated stir bar was charged with (2E,4E)-2-chloro-5-(pyrrolidin-1-yl)hexa-2,4-dienedinitrile (7a) (48.8 mg, $200 \mu \mathrm{mol}, 1.00$ equiv). Phenylboronic acid (48.8 mg, $0.400 \mathrm{mmol}, 2.00$ equiv), Bis(dibenzylideneacetone)palladium(0) (5.70 mg, $9.90 \mu \mathrm{mol}, 5.0 \mathrm{~mol} \%$ ), BrettPhos (21.0 mg, $39.0 \mu \mathrm{mol}, 20.0 \mathrm{~mol} \%$ ), cesium carbonate (130.0 mg, 400 $\mu$ mol, 2.00 equiv) were added, followed by 1,4-dioxane ( $1 \mathrm{~mL}, 0.2 \mathrm{M})$. The vial was heated to $60{ }^{\circ} \mathrm{C}$ in an $\mathrm{Al}$ heating block and stirred for 12 hours. The mixture was cooled to room temperature and quenched with water $(2 \mathrm{~mL})$, and extracted with ethyl acetate $(3 \times 2 \mathrm{~mL})$. The organic layer was dried with $\mathrm{Na}_{2} \mathrm{SO}_{4}$, and concentrated in vacuo, to yield a brown solid. The crude residue was purified via column chromatography on silica gel using 90:10 hexanes:ethyl acetate as the eluent. This procedure afforded the title compound as an orange solid $(37.2 \mathrm{mg}, 75 \%, 149 \mu \mathrm{mol})$. The compound was obtained as a 10:1 mixture of diastereomers as judged by ${ }^{1} \mathrm{H}$ NMR analysis. Data are for the major isomer.

(From Br compound) A flame-dried $4 \mathrm{~mL}$ dram vial equipped with a septum cap and a magnetic Teflon ${ }^{\circledR}$ coated stir bar was charged with (2E,4E)-2-bromo-5-(pyrrolidin-1-yl)hexa-2,4-dienedinitrile (7b) (48.8 $\mathrm{mg}, \quad 200 \mu \mathrm{mol}, 1.00$ equiv). Phenylboronic acid (48.8 mg, $400 \mu \mathrm{mmol}, 2.00$ equiv), Bis(dibenzylideneacetone)palladium(0) $(5.70 \mathrm{mg}, 9.90 \mu \mathrm{mol}, 5.0 \mathrm{~mol} \%)$, BrettPhos $(21.0 \mathrm{mg}, 39.0 \mu \mathrm{mol}$, $20.0 \mathrm{~mol} \%$ ), cesium carbonate ( $130.0 \mathrm{mg}, 400 \mu \mathrm{mol}, 2.00$ equiv) were added, followed by 1,4-dioxane (1.0 $\mathrm{mL}, 0.20 \mathrm{M}$ ). The vial was heated to $60^{\circ} \mathrm{C}$ in an $\mathrm{Al}$ heating block and stirred for 12 hours. The mixture was cooled to room temperature and quenched with water $(2 \mathrm{~mL})$, extracted with ethyl acetate $(3 \times 2 \mathrm{~mL})$. The organic layer was dried with $\mathrm{Na}_{2} \mathrm{SO}_{4}$, and concentrated in vacuo, to yield a brown solid. The crude residue was purified via column chromatography on silica gel using 90:10 hexanes:ethyl acetate as the eluent. This procedure afforded the title compound of as an orange solid $(42.6 \mathrm{mg}, 86 \%, 171 \mu \mathrm{mol})$. The compound was obtained as a 10:1 mixture of diastereomers as judged by ${ }^{1} \mathrm{H}$ NMR analysis. Data are for the major isomer.

m.p. $177-179{ }^{\circ} \mathrm{C}\left(\mathrm{CDCl}_{3}\right)$.

$\mathbf{R}_{\mathbf{f}}(90: 10$ hexanes:ethyl acetate $)=0.26$.

${ }^{1}$ H NMR $\left(600 \mathrm{MHz}, \mathrm{CDCl}_{3}\right)$ 8 7.59-7.49 (m, 3H), 7.42-7.32 (m, 2H), 7.32-7.24 (m, 1H), 6.01 (d, $J=12.0$ $\mathrm{Hz}, 1 \mathrm{H}), 3.51-3.40$ (m, 4H), 2.08-1.99 (m, 4H). 
${ }^{13}$ C NMR $\left(151 \mathrm{MHz}, \mathrm{CDCl}_{3}\right) \delta 138.0,133.9,129.0,128.0,126.2,125.0,118,113.4,107.5,106.2,49.8$, 25.3 .

IR (ATR) 2979, 2954, 2923, 2853, 2225, 2201, 1603, $1576 \mathrm{~cm}^{-1}$.

HRMS $\left(\right.$ ESI $\left.^{+}\right) \mathrm{m} / \mathrm{z}$ : $[\mathrm{M}+]$ Calcd for $\mathrm{C}_{16} \mathrm{H}_{15} \mathrm{~N}_{3}$ 249.1266; Found 249.1265.

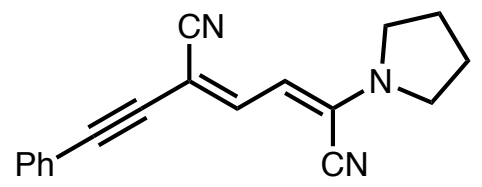

(2Z,4E)-2-(Phenylethynyl)-5-(pyrrolidin-1-yl)hexa-2,4-dienedinitrile (29). (From Cl compound) A flame-dried $4 \mathrm{~mL}$ vial equipped with a septum cap and a magnetic Teflon ${ }^{\circledR}$-coated stir bar was charged with (2E,4E)-2-chloro-5-(pyrrolidin-1-yl)hexa-2,4-dienedinitrile (7a) (41.4 mg, $199 \mu$ mol, 1.00 equiv). Tetrakis(triphenylphosphine)palladium(0) $(16.2 \mathrm{mg}, 14.0 \mu \mathrm{mol}, 7.00 \mathrm{~mol} \%)$ and copper (I) iodide (4.60 $\mathrm{mg}, 24.0 \mu \mathrm{mol}, 12.0 \mathrm{~mol} \%)$ were added followed by 1,4-dioxane (2.0 mL, $0.10 \mathrm{M})$. Phenylacetylene (100 $\mu \mathrm{L}, \rho=0.930 \mathrm{~g} / \mathrm{mL}, 920 \mu \mathrm{mol}, 4.60$ equiv) and triethylamine $(120 \mu \mathrm{L}, \rho=0.725 \mathrm{~g} / \mathrm{mL}, 880 \mu \mathrm{mol}, 4.60$ equiv) were added and the vial was heated to $60{ }^{\circ} \mathrm{C}$ in an $\mathrm{Al}$ heating block and stirred for 12 hours. The mixture was cooled to room temperature and concentrated to yield brown oil. The crude residue was purified via column chromatography on silica gel using 90:10 hexanes:ethyl acetate as the eluent. This procedure afforded the title compound as a brown oil $(36.7 \mathrm{mg}, 67 \%, 134 \mu \mathrm{mol})$. The compound was obtained as a 4:1 mixture of diastereomers as judged by ${ }^{1} \mathrm{H}$ NMR analysis. Data are for the major isomer.

(From Br compound) A flame-dried $4 \mathrm{~mL}$ vial equipped with a septum cap and a magnetic Teflon ${ }^{\circledR}$ coated stir bar was charged with (2E,4E)-2-bromo-5-(pyrrolidin-1-yl)hexa-2,4-dienedinitrile (7b) (23.7 mg, $94.4 \mu \mathrm{mol}, 1.00$ equiv). Tetrakis(triphenylphosphine)palladium(0) $(8.20 \mathrm{mg}, 8.20 \mu \mathrm{mol}, 7.00 \mathrm{~mol} \%)$ and copper (I) iodide (3.10 mg, $12.0 \mu \mathrm{mol}, 12 \mathrm{~mol} \%)$ were added followed by 1,4-dioxane (1.0 mL, $0.10 \mathrm{M})$. Phenylacetylene $(50.0 \mu \mathrm{L}, \rho=0.930 \mathrm{~g} / \mathrm{mL}, 460 \mu \mathrm{mol}, 4.6$ equiv $)$ and triethylamine $(60 \mu \mathrm{L}, \rho=0.725 \mathrm{~g} / \mathrm{mL}$, $440 \mu \mathrm{mol}, 4.60$ equiv) were added and the vial was heated to $60^{\circ} \mathrm{C}$ in an $\mathrm{Al}$ heating block and stirred for 12 hours. The mixture was cooled to room temperature and concentrated to yield brown oil. The crude residue was purified via column chromatography on silica gel using 90:10 hexanes:ethyl acetate as the eluent. This procedure afforded the title compound as a brown oil $(22.2 \mathrm{mg}, 86 \%, 81.3 \mu \mathrm{mol})$. The compound was obtained as a 4:1:1 mixture of isomers (product isomerizes on silica gel) as judged by ${ }^{1} \mathrm{H}$ NMR analysis. Data are for the major isomer.

$\mathbf{R}_{\mathbf{f}}(90: 10$ hexanes:ethyl acetate $)=0.10$.

${ }^{1}$ H NMR $\left(400 \mathrm{MHz}, \mathrm{CDCl}_{3}\right) \delta$ 7.47-7.43 (m, 2H), 7.37 (d, $\left.J=12.4 \mathrm{~Hz}, 1 \mathrm{H}\right), 7.35-7.31(\mathrm{~m}, 3 \mathrm{H}), 5.90(\mathrm{~d}, J$ $=12.4 \mathrm{~Hz}, 1 \mathrm{H}), 3.51(\mathrm{~d}, J=6.4 \mathrm{~Hz}, 4 \mathrm{H}), 2.09-2.02(\mathrm{~m}, 4 \mathrm{H})$. 
${ }^{13}$ C NMR $\left(151 \mathrm{MHz}, \mathrm{CDCl}_{3}\right) \delta 147.8,131.4,128.6,128.4,126.8,122.4,116.4,112.8,106.4,92.6,86.4$, $85.0,50.0,25.2$.

IR (ATR) 2954, 2923, 2854, 2362, 2226, 2207, 2032, $1568 \mathrm{~cm}^{-1}$.

HRMS (APCI) m/z: [M+H+] Calcd for $\mathrm{C}_{18} \mathrm{H}_{16} \mathrm{~N}_{3}$ 274.1344; Found 274.1338.

\section{Preparation and Characterization of Interrupted Addition to 3b Products}

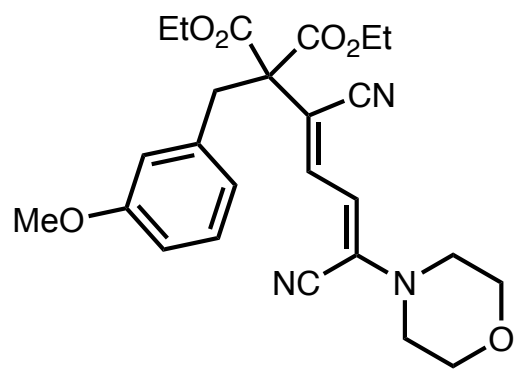

2-((1Z,3E)- Diethyl -1,4-dicyano-4-morpholinobuta-1,3-dien-1-yl)-2-(3-methoxybenzyl)malonate (26). A flame dried $4 \mathrm{~mL}$ vial equipped with a septum cap, and magnetic Teflon®-coated stir bar was charged with diethyl 2-(3-methoxybenzyl)malonate (S9) (67.0 mg, $240 \mu \mathrm{mol}, 1.20$ equiv), THF (0.50 mL), and sodium hydride (60\% in mineral oil, $10.0 \mathrm{mg}, 250 \mu \mathrm{mol}, 1.30$ equiv). To the mixture $(2 E, 4 E)-2,5-$ dibromohexa-2,4-dienedinitrile (3b) (52.4 mg, $198 \mu \mathrm{mol}, 1.00$ equiv) in THF (0.50 mL) was added and stirred at $25^{\circ} \mathrm{C}$ for 35 minutes. Morpholine $(200 \mu \mathrm{L}, \rho=1.01 \mathrm{~g} / \mathrm{mL}, 2.00 \mathrm{mmol}, 10.0$ equiv) was added, and the reaction stirred at $25{ }^{\circ} \mathrm{C}$ for 12 hours. The mixture was concentrated to yield an orange oil. The crude residue was purified via column chromatography on silica gel using 70:30 hexanes:ethyl acetate as the eluent. This procedure afforded the title compound as a yellow solid $(75.4 \mathrm{mg}, 82 \%, 161 \mu \mathrm{mol})$.

m.p. $92-95{ }^{\circ} \mathrm{C}\left(\mathrm{CDCl}_{3}\right)$.

$\mathbf{R}_{\mathbf{f}}(70: 30$ hexanes:ethyl acetate $)=0.18$.

${ }^{1}$ H NMR $\left(600 \mathrm{MHz}, \mathrm{CDCl}_{3}\right) \delta$ 7.27-7.20 (m, 1H), 7.17 (t, $\left.J=7.9 \mathrm{~Hz}, 1 \mathrm{H}\right), 6.81-6.70(\mathrm{~m}, 3 \mathrm{H}), 6.14(\mathrm{~d}, J$ $=11.7 \mathrm{~Hz}, 1 \mathrm{H}), 4.30-4.19(\mathrm{~m}, 4 \mathrm{H}), 3.78-3.71(\mathrm{~m}, 7 \mathrm{H}), 3.52(\mathrm{~s}, 2 \mathrm{H}), 3.29(\mathrm{dd}, J=5.9,4.0 \mathrm{~Hz}, 4 \mathrm{H}), 1.26(\mathrm{t}$, $J=7.1 \mathrm{~Hz}, 6 \mathrm{H})$.

${ }^{13}$ C NMR $\left(151 \mathrm{MHz}, \mathrm{CDCl}_{3}\right) \delta 168.0,159.4,143.9,136.1,130.3,129.3,122.6,116.8,116.1,112.8,112.4$, $109.8,106.7,65.7,62.6,61.9,55.2,48.2,40.6,13.9$.

IR (ATR) 2978, 2936, 2903, 2856, 2229, 2203, 1733, 1583, $1564 \mathrm{~cm}^{-1}$.

HRMS (ESI ${ }^{+}$) m/z: [M+H $\left.{ }^{+}\right]$Calcd for $\mathrm{C}_{25} \mathrm{H}_{30} \mathrm{~N}_{3} \mathrm{O}_{6}$ 468.2135; Found 468.2123. 


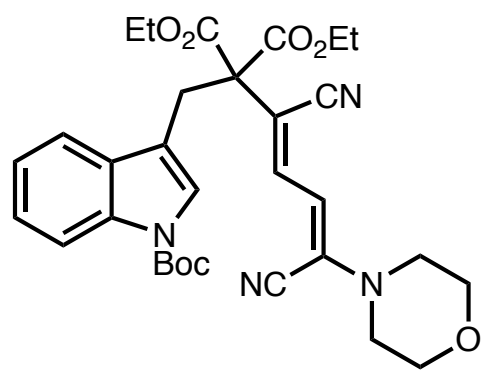

Diethyl 2-((1-(tert-butoxycarbonyl)-1H-indol-3-yl)methyl)-2-((1Z,3E)-1,4-dicyano-4morpholinobuta-1,3-dien-1-yl)malonate (27). A flame dried $4 \mathrm{~mL}$ vial equipped with a septum cap, and magnetic Teflon ${ }^{\circledR}$-coated stir bar was charged with diethyl 2-((1-(tert-butoxycarbonyl)-1H-indol-3yl)methyl)malonate (S7) $(93.0 \mathrm{mg}, 240 \mu \mathrm{mol}, 1.20$ equiv), THF $(0.50 \mathrm{~mL})$, sodium hydride $(60 \%$ in mineral oil, $10.0 \mathrm{mg}, 250 \mu \mathrm{mol}, 1.30$ equiv). (2E,4E)-2,5-dibromohexa-2,4-dienedinitrile (3b) (52.4 mg, $200 \mu \mathrm{mol}$, 1.00 equiv) in THF $(0.50 \mathrm{~mL})$ was added to the mixture and the mixture stirred at $25{ }^{\circ} \mathrm{C}$ for 40 minutes. Morpholine $(0.20 \mathrm{~mL}, \rho=1.01 \mathrm{~g} / \mathrm{mL}, 2.00 \mathrm{mmol}, 10.0$ equiv) was then added, and the reaction stirred at $25^{\circ} \mathrm{C}$ for 5 hours. The mixture was concentrated in vacuo to yield orange oil. The crude residue was purified via column chromatography on silica gel using 80:20 hexanes:ethyl acetate as the eluent. This procedure afforded the title compound as an orange low melting solid $(82.1 \mathrm{mg}, 71 \%, 142 \mu \mathrm{mol})$.

m.p. $48-51{ }^{\circ} \mathrm{C}\left(\mathrm{CDCl}_{3}\right)$.

$\mathbf{R}_{\mathbf{f}}(70: 30$ hexanes:ethyl acetate $)=0.38$.

${ }^{1}$ H NMR $\left(600 \mathrm{MHz}, \mathrm{CDCl}_{3}\right) \delta 8.07(\mathrm{~s}, 1 \mathrm{H}), 7.51-7.43(\mathrm{~m}, 2 \mathrm{H}), 7.30-7.15(\mathrm{~m}, 3 \mathrm{H}), 6.08(\mathrm{~d}, J=11.7 \mathrm{~Hz}$, $1 \mathrm{H}), 4.25-4.14(\mathrm{~m}, 4 \mathrm{H}), 3.77-3.71(\mathrm{~m}, 4 \mathrm{H}), 3.63(\mathrm{~s}, 2 \mathrm{H}), 3.28-3.21(\mathrm{~m}, 4 \mathrm{H}), 1.65(\mathrm{~s}, 9 \mathrm{H}), 1.22(\mathrm{t}, J=7.1$ $\mathrm{Hz}, 6 \mathrm{H})$.

${ }^{13}$ C NMR (151 MHz, $\left.\mathrm{CDCl}_{3}\right) \delta 168.1,149.6,144.0,134.9,131.0,130.3,125.2,124.4,122.4,118.9,116.8$, $115.2,113.7,112.2,109.7,106.6,83.7,65.7,62.7,61.4,48.2,29.9,28.2,13.8$.

IR (ATR) 3055, 2980, 2935, 2860, 2227, 2204, 1731, 1585, $1563 \mathrm{~cm}^{-1}$.

HRMS (ESI ${ }^{+}$) m/z: $\left[\mathrm{M}+\mathrm{H}^{+}\right]$Calcd for $\mathrm{C}_{31} \mathrm{H}_{37} \mathrm{~N}_{4} \mathrm{O}_{7}$ 577.2663; Found 577.2649.

\section{Preparation and Characterization of Annulation Reaction Products}




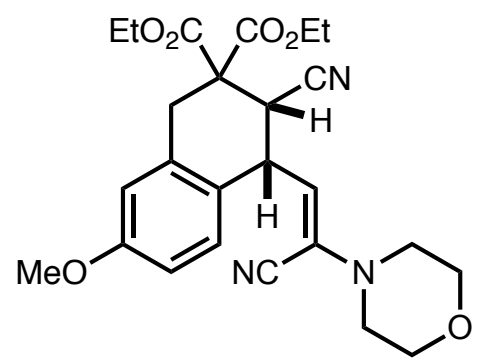

(3S,4R)- Diethyl-3-cyano-4-((E)-2-cyano-2-morpholinovinyl)-7-methoxy-3,4-dihydronaphthalene2,2(1H)-dicarboxylate (30). A flame dried $4 \mathrm{~mL}$ vial equipped with a magnetic Teflon ${ }^{\circledR}$-coated stir bar was charged with 2-((1Z,3E)- diethyl -1,4-dicyano-4-morpholinobuta-1,3-dien-1-yl)-2-(3methoxybenzyl)malonate (26, $59.8 \mathrm{mg}, 128 \mu \mathrm{mol}, 1.00$ equiv) aluminum trichloride $(15.5 \mathrm{mg}, 116 \mu \mathrm{mol}$, 0.90 equiv) and 1,2-dichloroethane $(1.0 \mathrm{~mL}, 0.10 \mathrm{M})$. The reaction was heated to $60{ }^{\circ} \mathrm{C}$ in an $\mathrm{Al}$ heating block and stirred for 2 hours. The reaction was basified with saturated sodium bicarbonate $(2.0 \mathrm{~mL})$, ammonium hydroxide $(0.50 \mathrm{~mL})$ and extracted with dichloromethane $(3 \times 2.0 \mathrm{~mL})$. The organic layer was dried with $\mathrm{Na}_{2} \mathrm{SO}_{4}$, and concentrated in vacuo to yield a brown oil as a 75:25 ratio of diastereomers. The crude residue was purified via column chromatography on silica gel using 80:20 to 70:30 hexanes:ethyl acetate as the eluent. This procedure afforded the two diastereomers $(52.9 \mathrm{mg}, 88 \%, 113.3 \mu \mathrm{mol})$ as described below:

Data for the major diastereomer:

pale yellow oil $(40.1 \mathrm{mg}, 67 \%, 85.9 \mu \mathrm{mol})$

$\mathbf{R}_{\mathbf{f}}(70: 30$ hexanes:ethyl acetate $)=0.37$.

${ }^{1} \mathbf{H}$ NMR $\left(600 \mathrm{MHz}, \mathrm{CDCl}_{3}\right) \delta 6.97(\mathrm{dd}, J=8.4,1.0 \mathrm{~Hz}, 1 \mathrm{H}), 6.78-6.72(\mathrm{~m}, 2 \mathrm{H}), 5.35(\mathrm{~d}, J=10.0 \mathrm{~Hz}, 1 \mathrm{H})$, $4.37-4.26(\mathrm{~m}, 3 \mathrm{H}), 4.26-4.21(\mathrm{~m}, 1 \mathrm{H}), 4.19(\mathrm{dd}, J=10.8,7.1 \mathrm{~Hz}, 1 \mathrm{H}), 4.12(\mathrm{dd}, J=10.1,5.4 \mathrm{~Hz}, 1 \mathrm{H}), 3.94$ $(\mathrm{dd}, J=5.4,1.7 \mathrm{~Hz}, 1 \mathrm{H}), 3.79(\mathrm{~d}, J=1.7 \mathrm{~Hz}, 7 \mathrm{H}), 3.69-3.62(\mathrm{~m}, 1 \mathrm{H}), 3.39$ (dd, $J=17.2,1.5 \mathrm{~Hz}, 1 \mathrm{H}), 3.08$ (q, $J=5.1 \mathrm{~Hz}, 4 \mathrm{H}), 1.33(\mathrm{t}, J=7.1 \mathrm{~Hz}, 3 \mathrm{H}), 1.24(\mathrm{t}, J=7.1 \mathrm{~Hz}, 3 \mathrm{H})$.

${ }^{13}$ C NMR $\left(151 \mathrm{MHz}, \mathrm{CDCl}_{3}\right) \delta 167.9,166.9,159.1,133.7,129.1,128.5,124.3,117.7,117.3,113.8,113.62$, $113.57,66.0,63.2,62.9,55.32,55.30,48.9,38.9,37.6,31.8,14.0,13.8$.

IR (ATR) 2979, 2906, 2838, 2243, 2225, 1734, 1610, 1577, $1502 \mathrm{~cm}^{-1}$.

HRMS (APCI) m/z: [M+H+] Calcd for $\mathrm{C}_{25} \mathrm{H}_{30} \mathrm{~N}_{3} \mathrm{O}_{6} 468.2135$; Found 468.2125. 


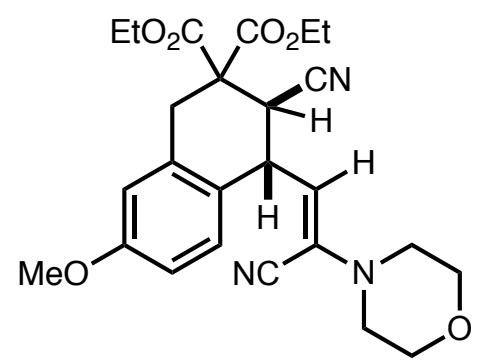

(3S,4S)-Diethyl-3-cyano-4-((E)-2-cyano-2-morpholinovinyl)-7-methoxy-3,4-dihydronaphthalene2,2(1H)-dicarboxylate (S10).

Data for the minor diastereomer:

pale yellow oil (12.8 mg, 21\%, $27.4 \mu \mathrm{mol})$

$\mathbf{R}_{\mathbf{f}}(70: 30$ hexanes:ethyl acetate $)=0.11$.

${ }^{1}$ H NMR $\left(600 \mathrm{MHz}, \mathrm{CDCl}_{3}\right) \delta 7.00(\mathrm{dd}, J=8.6,1.0 \mathrm{~Hz}, 1 \mathrm{H}), 6.76(\mathrm{dd}, J=8.7,2.7 \mathrm{~Hz}, 1 \mathrm{H}), 6.65(\mathrm{~d}, J=$ $2.6 \mathrm{~Hz}, 1 \mathrm{H}), 5.00(\mathrm{~d}, J=10.3 \mathrm{~Hz}, 1 \mathrm{H}), 4.35-4.21(\mathrm{~m}, 5 \mathrm{H}), 4.17(\mathrm{t}, J=10.7 \mathrm{~Hz}, 2 \mathrm{H}), 3.82-3.73(\mathrm{~m}, 7 \mathrm{H})$, $3.56(\mathrm{~d}, J=16.5 \mathrm{~Hz}, 1 \mathrm{H}), 3.29(\mathrm{~d}, J=11.4 \mathrm{~Hz}, 1 \mathrm{H}), 3.18-3.11(\mathrm{~m}, 1 \mathrm{H}), 3.06(\mathrm{q}, J=4.6 \mathrm{~Hz}, 4 \mathrm{H}), 1.33(\mathrm{td}$, $J=7.1,1.6 \mathrm{~Hz}, 6 \mathrm{H})$.

${ }^{13}$ C NMR $\left(151 \mathrm{MHz}, \mathrm{CDCl}_{3}\right) \delta 169.2,167.2,159.2,133.0,129.5,125.0,118.0,117.0,113.69,113.68$, $66.0,63.0,62.9,55.6,55.3,48.9,40.8,38.7,35.8,14.0,13.8$.

IR (ATR) 3057, 2964, 2922, 2855, 2247, 2226, 1730, 1610, 1583, $1502 \mathrm{~cm}^{-1}$.

HRMS (APCI) m/z: [M+H+] Calcd for $\mathrm{C}_{25} \mathrm{H}_{30} \mathrm{~N}_{3} \mathrm{O}_{6} 468.2135$; Found 468.2126.

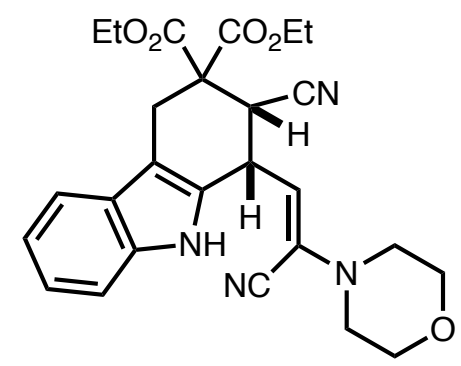

$1 R, 2 S$ - Diethyl -2-cyano-1-((E)-2-cyano-2-morpholinovinyl)-1,2,4,9-tetrahydro-3H-carbazole-3,3dicarboxylate (31). A flame dried $4 \mathrm{~mL}$ vial equipped with a magnetic Teflon ${ }^{\circledR}$-coated stir bar was charged with diethyl 2-((1-(tert-butoxycarbonyl)-1H-indol-3-yl)methyl)-2-((1Z,3E)-1,4-dicyano-4morpholinobuta-1,3-dien-1-yl)malonate (27). (52.7 mg, $910 \mu \mathrm{mol}, 1.0$ equiv) and 1,2-dichloroethane (1.0 $\mathrm{mL}, 0.10 \mathrm{M})$. The mixture was charged with trifluoroacetic acid $(50 \mu \mathrm{L}, \rho=1.49 \mathrm{~g} / \mathrm{mL}, 650 \mu \mathrm{mol}, 7.00$ equiv), and reaction stirred at $25^{\circ} \mathrm{C}$ for 14 hours. The mixture was quenched with saturated sodium 
bicarbonate $(2 \mathrm{~mL})$, ammonium hydroxide $(0.5 \mathrm{~mL})$ and extracted with dichloromethane $(3 \times 2 \mathrm{~mL})$. The organic layer was dried with $\mathrm{Na}_{2} \mathrm{SO}_{4}$, and concentrated in vacuo to yield brown oil as a $62: 38$ ratio of diastereomers. The crude residue was purified via column chromatography on silica gel using 80:20 to 70:30 hexanes:ethyl acetate as the eluent. This procedure afforded the combined diastereomers $(26.6 \mathrm{mg}$ $61 \%, 55.9 \mu \mathrm{mol})$ as described below:

Data for the major diastereomer:

orange solid (9.9 mg, 22\%, $20.8 \mu \mathrm{mol})$,

m.p. $72-75{ }^{\circ} \mathrm{C}\left(\mathrm{CDCl}_{3}\right)$.

${ }^{1}$ H NMR $\left(600 \mathrm{MHz}, \mathrm{CDCl}_{3}\right) \delta 7.77(\mathrm{~s}, 1 \mathrm{H}), 7.56(\mathrm{~d}, J=7.8 \mathrm{~Hz}, 1 \mathrm{H}), 7.31(\mathrm{~d}, J=8.1 \mathrm{~Hz}, 1 \mathrm{H}), 7.22-7.16$ (m, 1H), 7.16-7.11 (m, 1H), $5.37(\mathrm{~d}, J=10.3 \mathrm{~Hz}, 1 \mathrm{H}), 4.56-4.53(\mathrm{~m}, 1 \mathrm{H}), 4.40-4.26(\mathrm{~m}, 2 \mathrm{H}), 4.25-4.14$ $(\mathrm{m}, 2 \mathrm{H}), 4.11(\mathrm{dd}, J=5.6,1.1 \mathrm{~Hz}, 1 \mathrm{H}), 3.82-3.77(\mathrm{~m}, 4 \mathrm{H}), 3.73-3.63(\mathrm{~m}, 1 \mathrm{H}), 3.37$ (dd, $J=16.4,2.6 \mathrm{~Hz}$, $1 \mathrm{H}), 3.13(\mathrm{td}, J=4.5,2.8 \mathrm{~Hz}, 4 \mathrm{H}), 1.36$ (t, $J=7.1 \mathrm{~Hz}, 3 \mathrm{H}), 1.21(\mathrm{t}, J=7.1 \mathrm{~Hz}, 3 \mathrm{H})$.

${ }^{13}$ C NMR (151 MHz, $\left.\mathrm{CDCl}_{3}\right)$ 167.9, 167.0, 136.5, 129.2, 129.2, 127.0, 122.8, 120.1, 118.8, 117.5, 113.2, $112.7,110.9,108.1,66.0,63.1,63.0,56.8,48.7,38.1,35.6,24.5,14.2,13.9$.

$\mathbf{R}_{\mathbf{f}}(70: 30$ hexanes:ethyl acetate $)=0.22$.

IR (ATR) 3385, 2977, 2923, 2854, 2226, 2203, 1733, 1680, 1613, $1537 \mathrm{~cm}^{-1}$.

HRMS (APCI) m/z: [M+H'] Calcd for $\mathrm{C}_{26} \mathrm{H}_{29} \mathrm{~N}_{4} \mathrm{O}_{5}$ 477.2138; Found 477.2125.

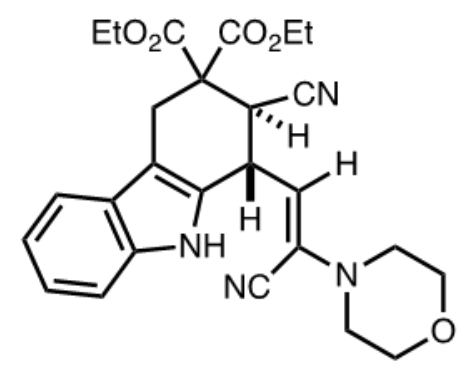

1R,2R- Diethyl -2-cyano-1-((E)-2-cyano-2-morpholinovinyl)-1,2,4,9-tetrahydro-3H-carbazole-3,3dicarboxylate (S11)

Data for the minor diastereomer:

orange solid $(6.7 \mathrm{mg}, 15 \%, 14.0 \mu \mathrm{mol})$

m.p. $122-125^{\circ} \mathrm{C}\left(\mathrm{CDCl}_{3}\right)$.

${ }^{1}$ H NMR $\left(600 \mathrm{MHz}, \mathrm{CDCl}_{3}\right) \delta 7.81(\mathrm{~s}, 1 \mathrm{H}), 7.48(\mathrm{~d}, J=7.9 \mathrm{~Hz}, 1 \mathrm{H}), 7.31(\mathrm{~d}, J=8.1 \mathrm{~Hz}, 1 \mathrm{H}), 7.20(\mathrm{t}, J=$ $8.2 \mathrm{~Hz}, 1 \mathrm{H}), 7.12(\mathrm{~d}, J=8.9 \mathrm{~Hz}, 1 \mathrm{H}), 5.00(\mathrm{~d}, J=10.3 \mathrm{~Hz}, 1 \mathrm{H}), 4.55-4.47$ (m, 1H), 4.38-4.29 (m, 3H), 
4.25-4.17 (m, 1H), $3.80(\mathrm{t}, J=4.9 \mathrm{~Hz}, 4 \mathrm{H}), 3.76(\mathrm{dd}, J=15.7,1.3 \mathrm{~Hz}, 2 \mathrm{H}), 3.42(\mathrm{~d}, J=10.5 \mathrm{~Hz}, 1 \mathrm{H}), 3.17-$ $3.07(\mathrm{~m}, 4 \mathrm{H}), 1.36(\mathrm{t}, J=7.1 \mathrm{~Hz}, 3 \mathrm{H}), 1.30(\mathrm{t}, J=7.1 \mathrm{~Hz}, 3 \mathrm{H})$.

${ }^{13}$ C NMR $\left(151 \mathrm{MHz}, \mathrm{CDCl}_{3}\right) \delta 169.4,167.3,136.4,129.7,129.5,126.6,123.0,120.2,118.6,117.8,113.3$, $113.1,111.0,107.3,65.9,63.1,62.8,56.8,48.7,39.2,38.0,28.1,14.0,13.8$.

$\mathbf{R}_{\mathbf{f}}(70: 30$ hexanes:ethyl acetate $)=0.11$.

IR (ATR) 3371, 3057, 2971, 2922, 2852, 2226, 2206, 1733, 1680, $1614 \mathrm{~cm}^{-1}$.

HRMS (APCI) m/z: [M+H+ $\mathrm{H}^{+}$Calcd for $\mathrm{C}_{26} \mathrm{H}_{29} \mathrm{~N}_{4} \mathrm{O}_{5}$ 477.2138; Found 477.2129.

Note: The remaining mass balance is accounted for in mixed fractions of the two diastereomers $(10.0 \mathrm{mg}$, $22 \%, 21.0 \mu \mathrm{mol})$.

\section{Descriptions of Stereochemical Assignments}

The geometry of diene 3a was determined by analysis of the ${ }^{1} \mathrm{H}$ coupled ${ }^{13} \mathrm{C}$ NMR spectrum. The relative stereochemistry of products $\mathbf{1 1 a}, \mathbf{3 0}, \mathbf{S 1 0}, \mathbf{3 1}, \mathbf{S 1 1}$ was assigned on the basis of ${ }^{1} \mathrm{H}$ NMR nOe studies. The data for these studies is provided below, along with the key nOe signals.

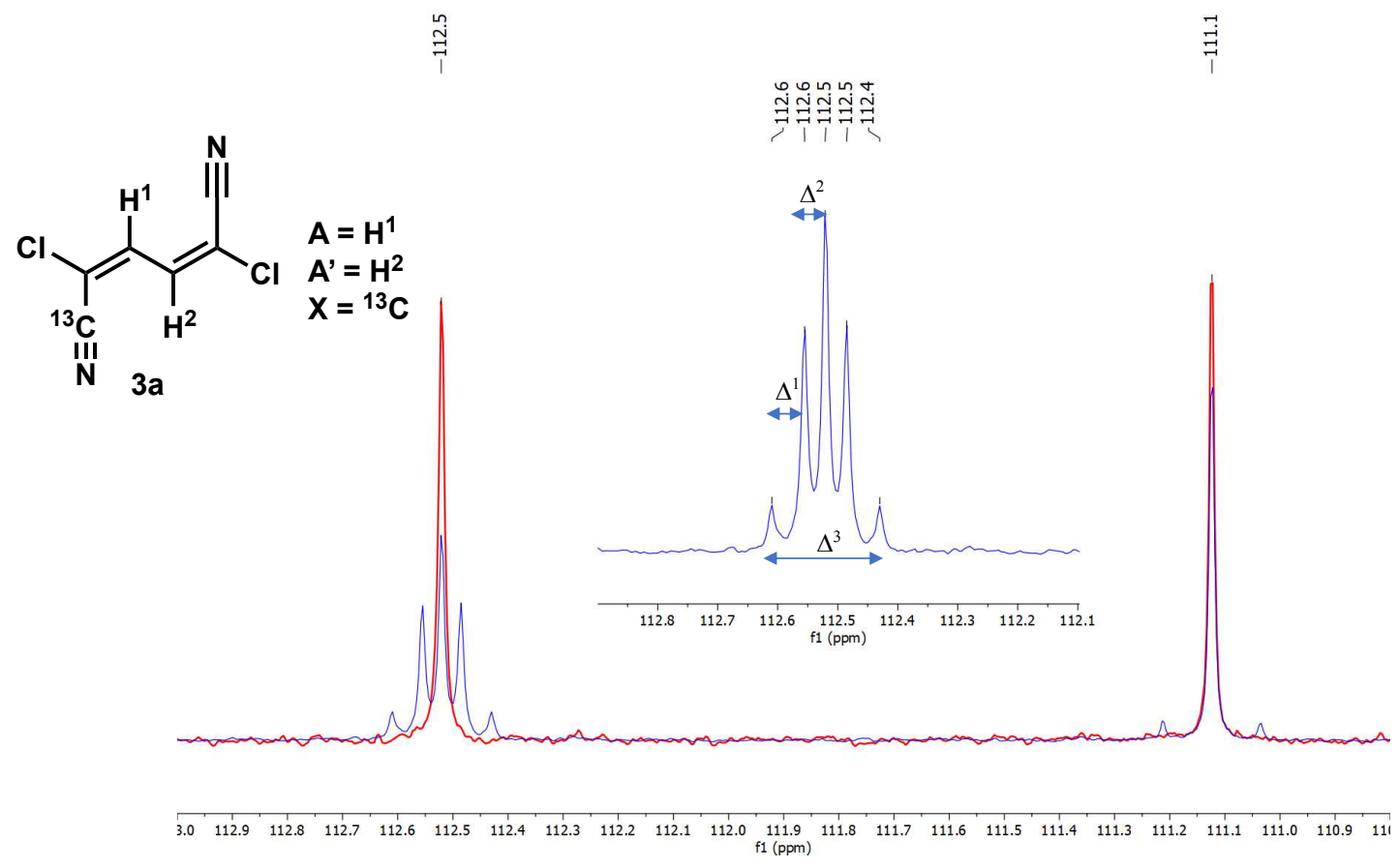

$\mathrm{CDCl}_{3} 151 \mathrm{MHz}(14.5 \mathrm{mg}$ 3a in $0.70 \mathrm{~mL} \mathrm{CDCl})$. ${ }^{1} \mathrm{H}$ decoupled ${ }^{13} \mathrm{C}$ spectrum shown in red. ${ }^{1} \mathrm{H}$ coupled ${ }^{13} \mathrm{C}$ spectrum shown in blue. 
The $2^{\text {nd }}$ order coupling pattern for the resonance at $112.5 \mathrm{ppm}$ (nitrile resonance) was simulated in SpinWorks ${ }^{9}$ software using the following parameters: $\mathrm{J}_{\mathrm{AX}}=+13 \mathrm{~Hz}, \mathrm{~J}_{\mathrm{A}^{\prime} \mathrm{X}}=-2 \mathrm{~Hz}, \mathrm{~J}_{\mathrm{AA}}{ }^{\prime}=+12 \mathrm{~Hz}, 2 \mathrm{~Hz}$ linewidth. Where A, A' and $\mathrm{X}$ are defined for 3a as shown above. This $J$-value is in accord with a transrelationship between ${ }^{1} \mathrm{H}$ and the nitrile group. ${ }^{10}$

\begin{tabular}{|l|l|}
\hline MEASURED FROM SPECTRUM & CALCULATED IN SPINWORKS \\
\hline$\Delta^{3}=27.2 \mathrm{~Hz}$ & $\Delta^{3}=27 \mathrm{~Hz}$ \\
\hline$\Delta^{2}=6.0 \mathrm{~Hz}$ & $\Delta^{2}=5.5 \mathrm{~Hz}$ \\
\hline$\Delta^{1}=7.6 \mathrm{~Hz}$ & $\Delta^{1}=8.4 \mathrm{~Hz}$ \\
\hline
\end{tabular}

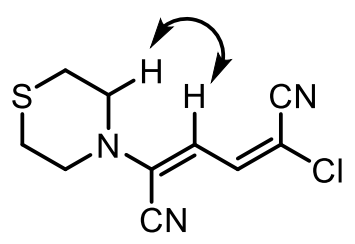

$11 \mathrm{a}$

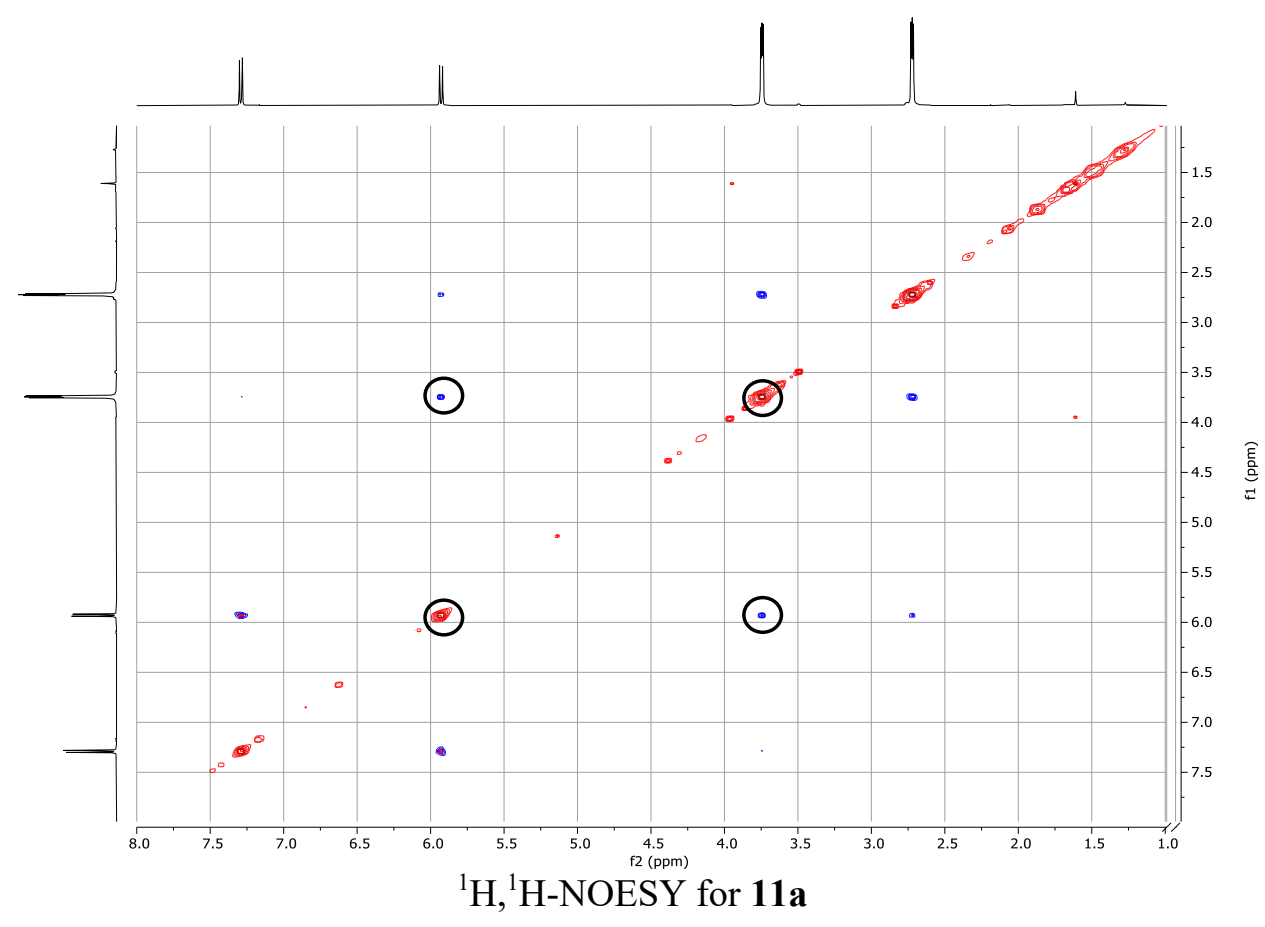



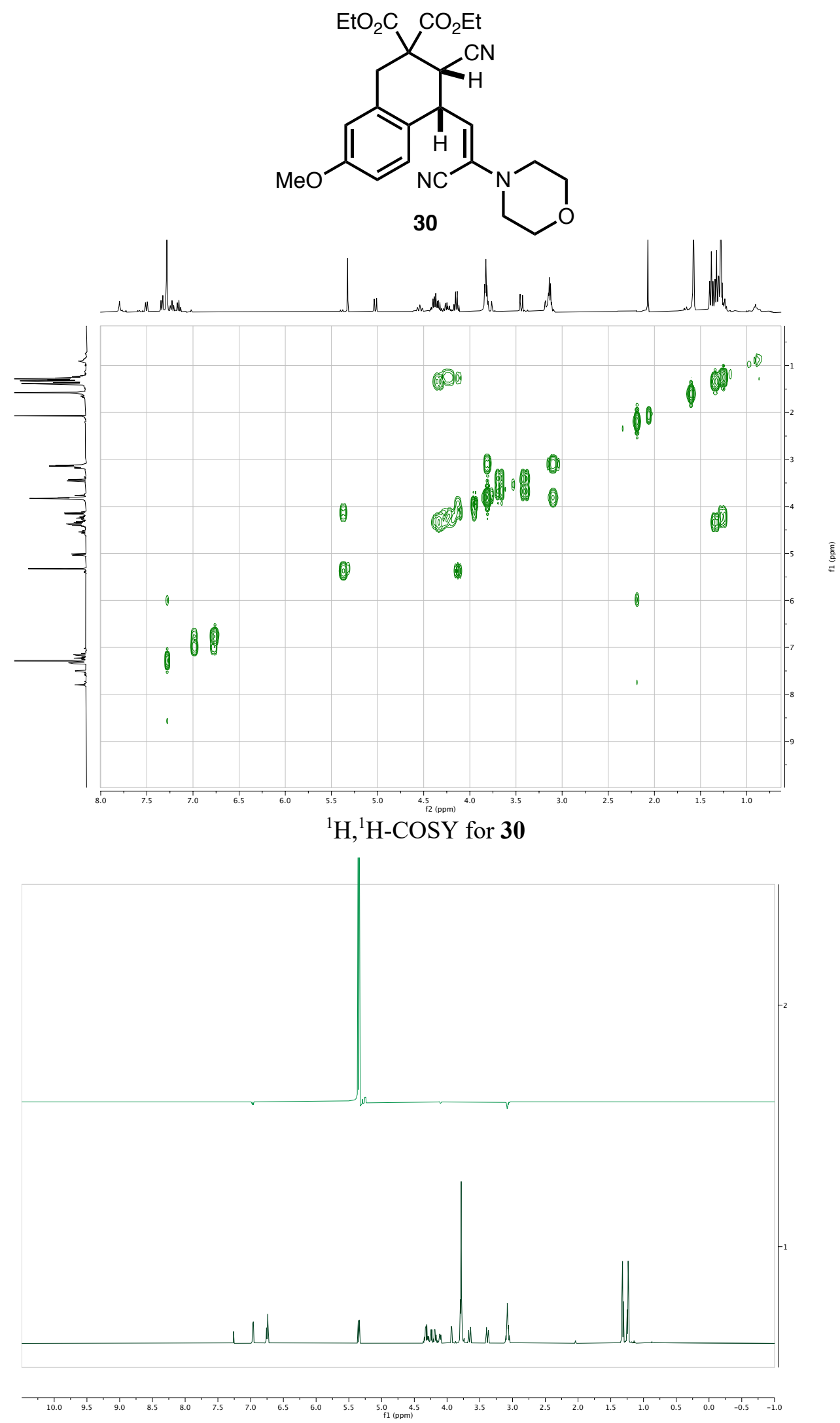

1D NOE for 30 irradiating peak at $5.36 \mathrm{ppm}$. No correlations observed 

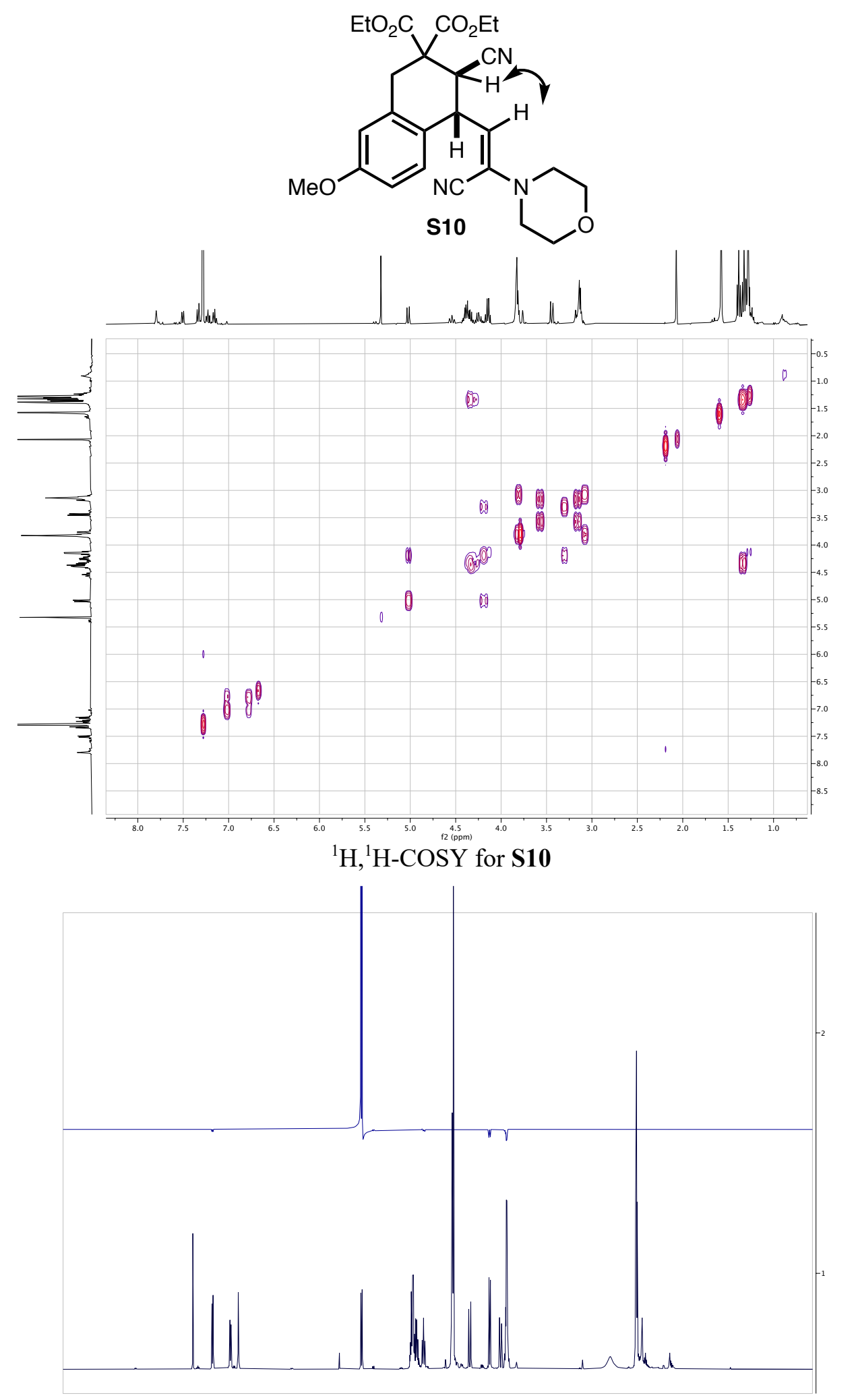

1D NOE for $\mathbf{S 1 0}$ irradiating peak at $5.00 \mathrm{ppm}$. Correlation seen with peak at $3.29 \mathrm{ppm}$, indicating they are on the same side of the cyclohexane. 


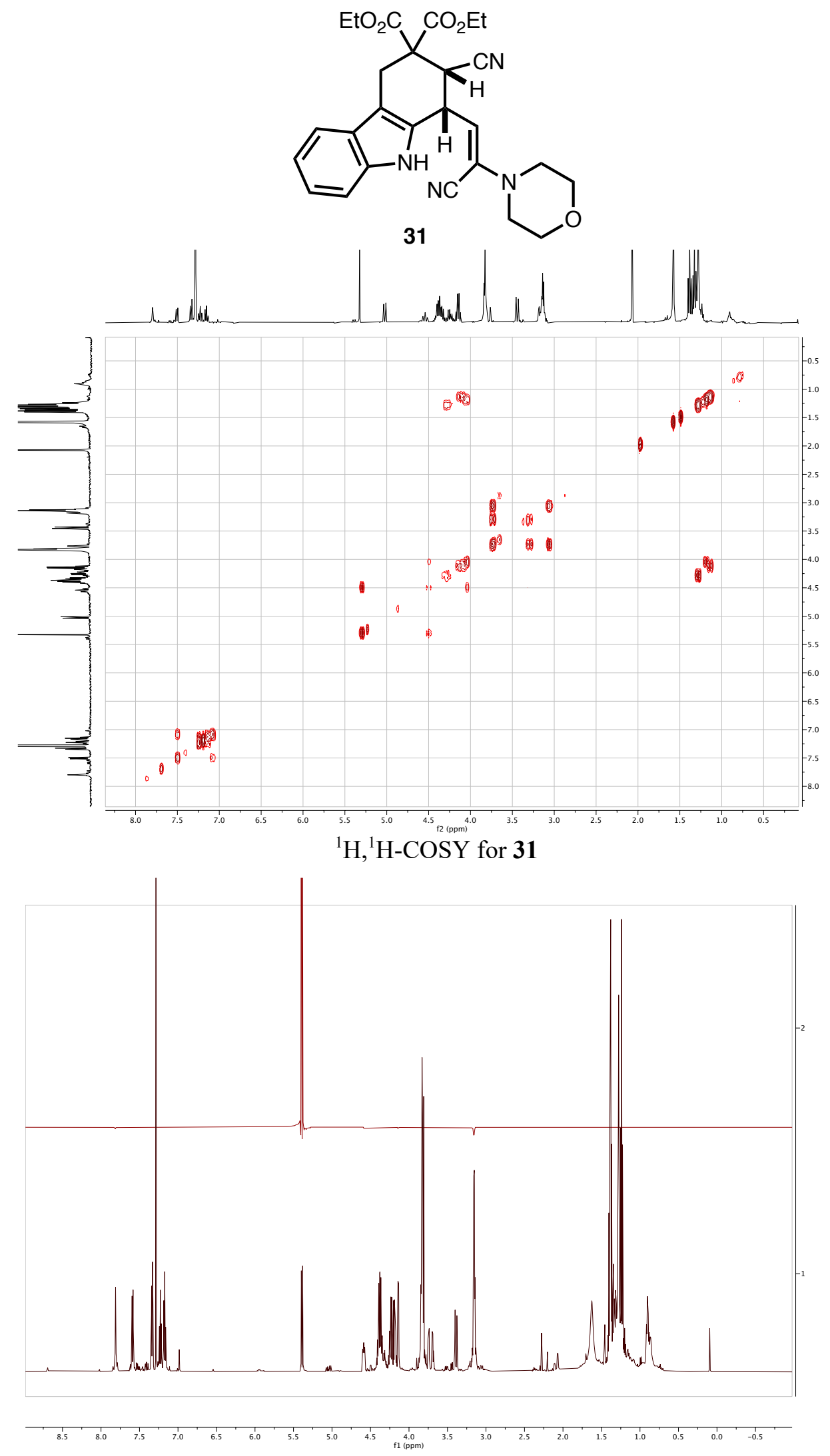

1D NOE for 31 irradiating peak at $5.39 \mathrm{ppm}$. No correlations observed 

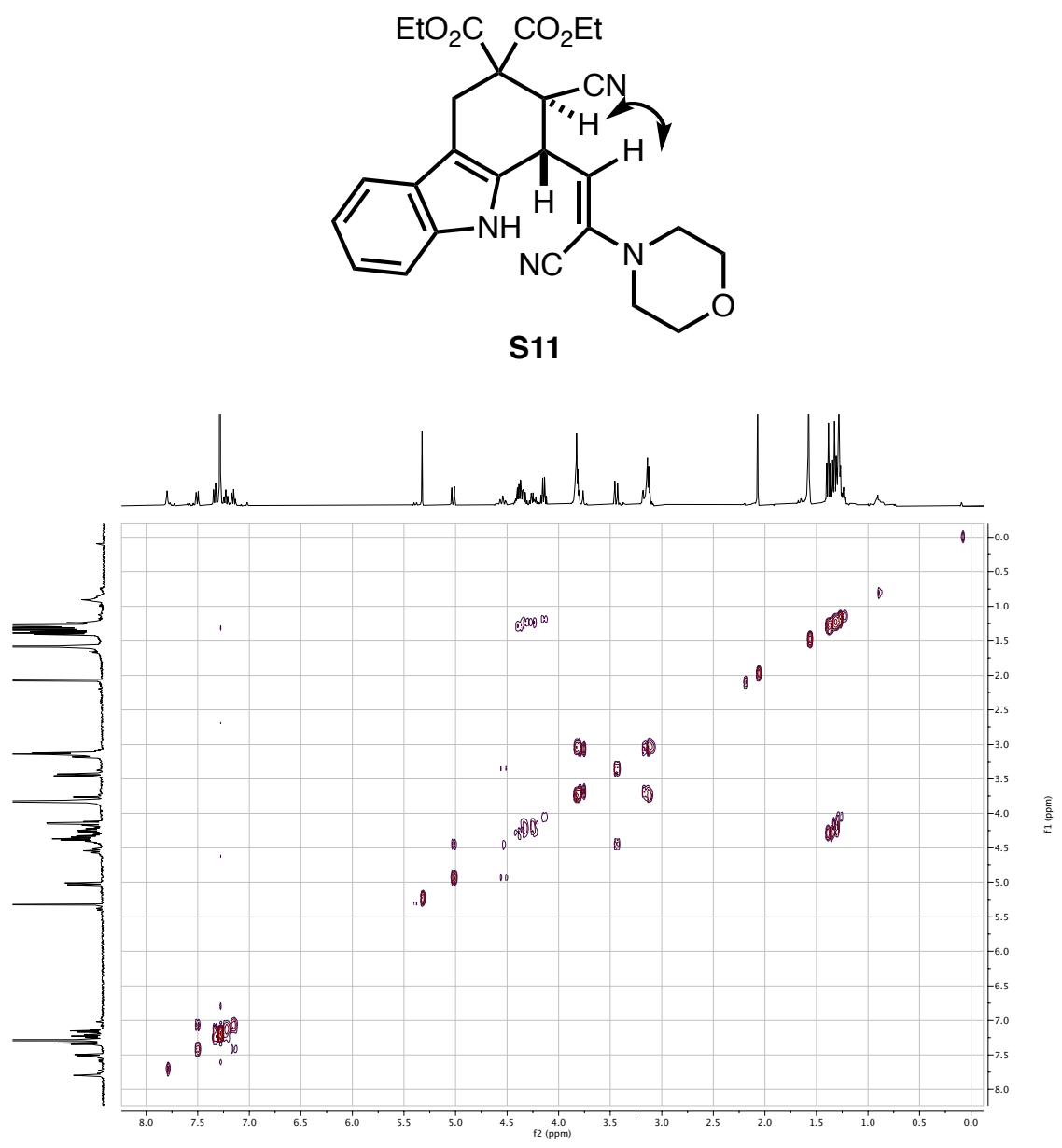

${ }^{1} \mathrm{H},{ }^{1} \mathrm{H}-\mathrm{COSY}$ for $\mathbf{S 1 1}$
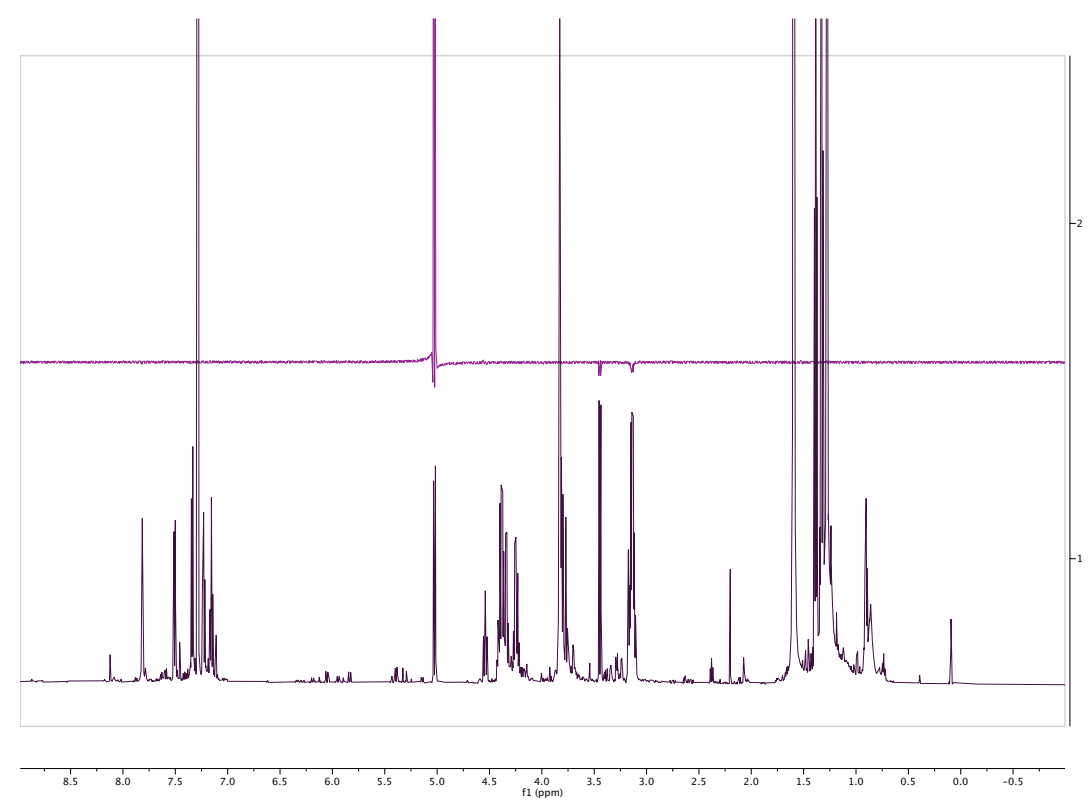

1D NOE for S11 irradiating peak at $5.01 \mathrm{ppm}$. Correlation seen with peak at $3.43 \mathrm{ppm}$, indicating they are on the same side of the cyclohexane. 


\section{X-ray Data for 3a and 19a}

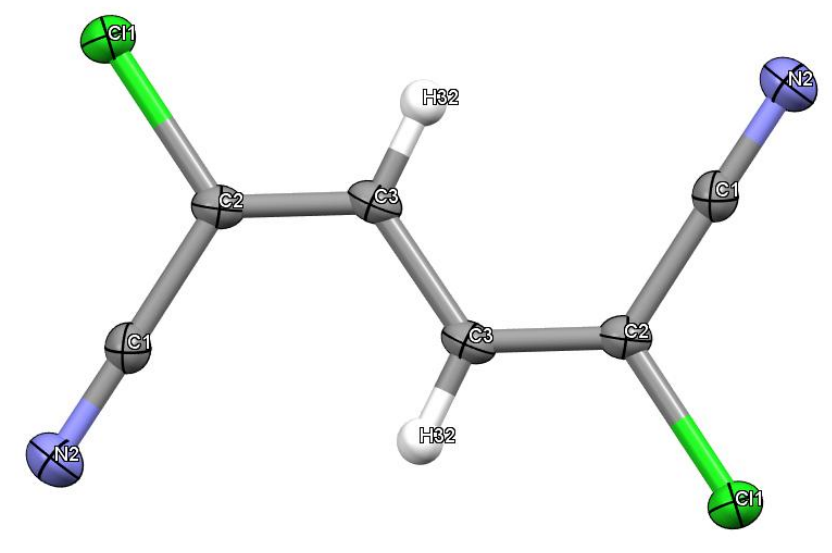

Figure S1: Molecular structure for 3a (50\% probability level shown).

X-ray quality crystals were grown by placing an uncapped 1 dram vial containing a $1.0 \mathrm{~mL}$ solution of compound 3a dissolved in ethyl acetate into a scintillation vial containing $2.0 \mathrm{~mL}$ of hexanes and allowing vapor diffusion to occur for 48 hours. A colorless crystal (approximate dimensions $0.200 \times 0.200 \times 0.200$ $\mathrm{mm}^{3}$ ) was placed onto the tip of MiTeGen and mounted on a Bruker SMART Apex II diffractometer and measured at $150 \mathrm{~K}$.

Data collection

A preliminary set of cell constants was calculated from reflections harvested from three sets of 12 frames. These initial sets of frames were oriented such that orthogonal wedges of reciprocal space were surveyed. This produced initial orientation matrices determined from 140 reflections. The data collection was carried out using $\mathrm{Cu} \mathrm{Ka}$ radiation (graphite monochromator) with a frame time of 10 seconds and a detector distance of $4.0 \mathrm{~cm}$. A randomly oriented region of reciprocal space was surveyed to achieve complete data with a redundancy of 4 . Sections of frames were collected with $0.50^{\circ}$ steps in $\mathrm{w}$ and $\mathrm{f}$ scans. Data to a resolution of $0.84 \AA$ were considered in the reduction. Final cell constants were calculated from the xyz centroids of 690 strong reflections from the actual data collection after integration (SAINT). The intensity data were corrected for absorption (SADABS). Please refer to Table 1 for additional crystal and refinement information.

Structure solution and refinement

The space group P -1 was determined based on intensity statistics and systematic absences. The structure was solved using Superflip3 and refined (full-matrix-least squares) using the Oxford University Crystals for Windows system. The charge-flipping solution provided most non-hydrogen atoms from the E-map. Full-matrix least squares / difference Fourier cycles were performed, which located the remaining nonhydrogen atoms. All non-hydrogen atoms were refined with anisotropic displacement parameters. The 
hydrogen atoms were placed in ideal positions and refined with individual relative isotropic displacement parameters.

Table S1. Crystal data and structure refinement for Compound 3a.

Empirical formula

Formula weight

Crystal color, shape, size

Temperature

Wavelength

Crystal system, space group

Unit cell dimensions

Volume

Z

Density (Calcd)

Absorption coefficient

$\mathrm{F}(000)$

Data collection

Diffractometer

Theta range for data collection

Index ranges

Reflections collected

Independent reflections

Observed Reflections
C6 H2 Cl2 N2

173.00

colorless plate fragment, $0.200 \times 0.200 \times 0.200 \mathrm{~mm}^{3}$

$150 \mathrm{~K}$

$1.54178 \AA$

Triclinic, P - 1

$\mathrm{a}=5.0796(2) \AA$

$\mathrm{a}=73.366(2)^{\circ}$.

$\mathrm{b}=6.0612(2) \AA \quad \mathrm{b}=69.663(2)^{\circ}$.

$\mathrm{c}=6.6441(2) \AA$

$178.113(11) \AA^{3}$

1

$1.613 \mathrm{Mg} / \mathrm{m}^{3}$

$7.505 \mathrm{~mm}^{-1}$

86.0

Bruker Apex Kappa Duo, Bruker

7.251 to $72.318^{\circ}$.

$-6<=\mathrm{h}<=6,-7<=\mathrm{k}<=7,-8<=1<=8$

3043

$690[\mathrm{R}(\mathrm{int})=0.026]$

689

multi-scan

0.22 and 0.22

Direct methods

Full-matrix least-squares on $\mathrm{F}^{2}$

$\mathrm{w}=1 /\left[\sigma^{2}\left(\mathrm{Fo}^{2}\right)+(\mathrm{AP})^{2}+\mathrm{BP}\right]$, with

$\mathrm{P}=\mathrm{A} \times \max \left(\mathrm{Fo}^{2}, 0\right)+\mathrm{CFc}^{2}, \mathrm{~A}=0.034, \mathrm{~B}=0.088, \mathrm{C}=$

$688 / 0 / 46$

1.0000

$\mathrm{R} 1=0.0257, \mathrm{wR} 2=0.0653$

$\mathrm{R} 1=0.0257, \mathrm{wR} 2=0.0653$

0.20 and -0.29 e. $\AA^{-3}$ 
Table S2. Atomic coordinates and equivalent isotropic displacement parameters $\left(\AA^{2}\right)$ for compound 3a. $\mathrm{U}(\mathrm{eq})$ is defined as one third of the trace of the orthogonalized $\mathrm{U}^{\mathrm{ij}}$ tensor.

\begin{tabular}{lllll} 
Label & $x$ & $y$ & $z$ & $\mathrm{U}_{\text {iso/equiv }}$ \\
\hline $\mathrm{C} 11$ & $0.10209(7)$ & $0.09722(6)$ & $0.19329(5)$ & 0.0238 \\
$\mathrm{~N} 2$ & $0.3108(3)$ & $0.2939(3)$ & $0.5714(2)$ & 0.0291 \\
$\mathrm{C} 1$ & $0.2921(3)$ & $0.4015(2)$ & 0.0195 \\
$\mathrm{C} 2$ & $0.3042(3)$ & $0.2882(2)$ & $0.1895(2)$ & 0.0182 \\
$\mathrm{C} 3$ & $0.2939(3)$ & $0.4194(2)$ & $0.0030(2)$ & 0.0189 \\
$\mathrm{H} 32$ & $0.4195(3)$ & $0.4030(2)$ & $-0.1290(2)$ & 0.0224 \\
\hline
\end{tabular}


Table S3. Bond lengths $[\AA]$ and angles $\left[^{\circ}\right]$ for compound 3a.

\begin{tabular}{llll}
\hline $\mathrm{C} 11-\mathrm{C} 2$ & $1.7263(14) \AA$ & $\mathrm{N} 2-\mathrm{C} 11$ & $1.144(2) \AA$ \\
$\mathrm{C} 1-\mathrm{C} 2$ & $1.4349(19) \AA$ & $\mathrm{C} 2-\mathrm{C} 3$ & $1.337(2) \AA$ \\
$\mathrm{C} 3-\mathrm{C} 32$ & $1.447(3) \AA$ & $\mathrm{C} 3-\mathrm{H} 32$ & $0.949 \AA$ \\
& & & \\
$\mathrm{N} 2-\mathrm{C} 1-\mathrm{C} 2$ & $179.41(16)^{\circ}$ & $\mathrm{C} 11-\mathrm{C} 2-\mathrm{C} 1$ & $114.13(11)^{\circ}$ \\
$\mathrm{C} 11-\mathrm{C} 2-\mathrm{C} 3$ & $121.85(11)^{\circ}$ & $\mathrm{C} 1-\mathrm{C} 2-\mathrm{C} 3$ & $124.02(13)^{\circ}$ \\
$\mathrm{C} 2-\mathrm{C} 3-\mathrm{C} 32$ & $122.54(15)^{\circ}$ & $\mathrm{C} 2-\mathrm{C} 3-\mathrm{H} 32$ & $117.469^{\circ}$ \\
$\mathrm{C} 32-\mathrm{C} 3-\mathrm{H} 32$ & $119.992^{\circ}$ & & \\
\hline
\end{tabular}

Table S4. Anisotropic displacement parameters $\left(\AA^{2}\right)$ for compound 3a. The anisotropic displacement factor exponent takes the form: $-2 \pi^{2}\left[h^{2} a^{* 2} U^{11}+\ldots+2 h k a^{*} b^{*} U^{12}\right]$

\begin{tabular}{lllllll} 
Label & $\mathrm{U}_{11}$ & $\mathrm{U}_{22}$ & $\mathrm{U}_{33}$ & $\mathrm{U}_{23}$ & $\mathrm{U}_{13}$ & $\mathrm{U}_{12}$ \\
\hline $\mathrm{C} 11$ & $0.0285(2)$ & $0.0262(2)$ & $0.0225(2)$ & $0.00450(15)$ & $0.00824(16)$ & $0.01334(17)$ \\
$\mathrm{N} 2$ & $0.0367(8)$ & $0.0360(8)$ & $0.0189(6)$ & $-0.0053(5)$ & $-0.0074(6)$ & $-0.0152(6)$ \\
$\mathrm{C} 1$ & $0.0212(7)$ & $0.0206(7)$ & $0.0170(7)$ & $-0.0029(5)$ & $-0.0031(5)$ & $-0.0085(6)$ \\
$\mathrm{C} 2$ & $0.0194(7)$ & $0.0194(7)$ & $0.0183(7)$ & $-0.0065(5)$ & $-0.0065(5)$ & $-0.0043(6)$ \\
$\mathrm{C} 3$ & $0.0216(7)$ & $0.0213(7)$ & $0.0158(7)$ & $-0.0073(5)$ & $-0.0066(5)$ & $-0.0033(6)$ \\
\hline
\end{tabular}




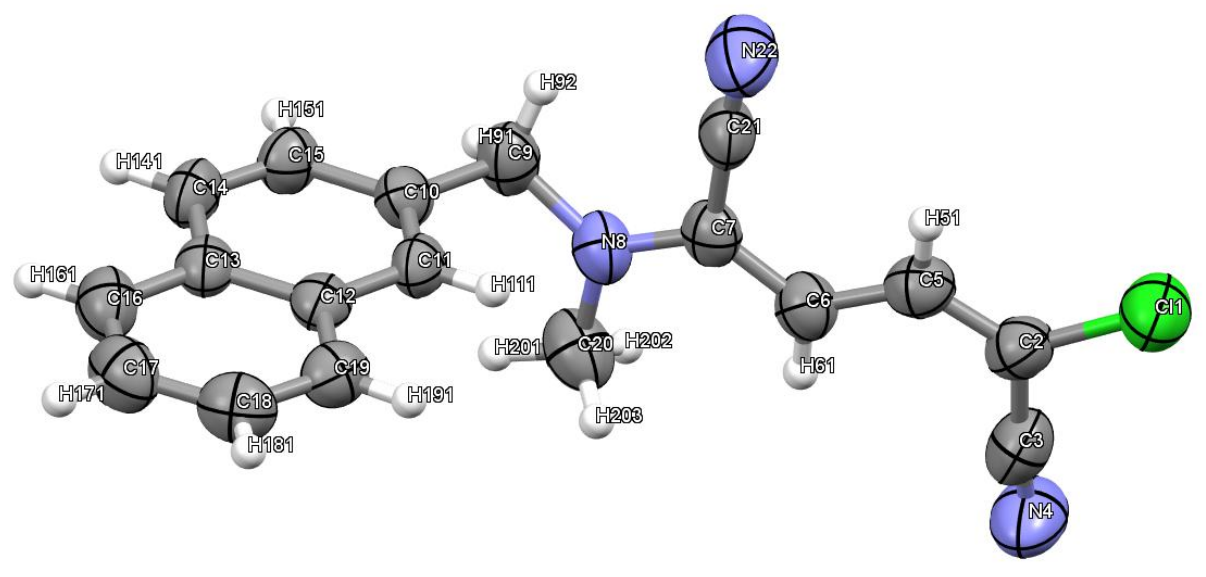

Figure S2: Molecular structure for 19a (50\% probability level shown).

X-ray quality crystals were grown by placing an uncapped 1 dram vial containing a $1.0 \mathrm{~mL}$ solution of compound 19a dissolved in ethyl acetate into a scintillation vial containing $2.0 \mathrm{~mL}$ of hexanes and allowing vapor diffusion to occur for 24 hours. A yellow crystal (approximate dimensions $0.200 \times 0.300 \times 0.300$ $\mathrm{mm}^{3}$ ) was placed onto the tip of MiTeGen and mounted on a Bruker SMART Apex II diffractometer and measured at $293 \mathrm{~K}$.

Data collection

A preliminary set of cell constants was calculated from reflections harvested from three sets of 12 frames. These initial sets of frames were oriented such that orthogonal wedges of reciprocal space were surveyed. This produced initial orientation matrices determined from 140 reflections. The data collection was carried out using $\mathrm{Cu} \mathrm{Ka}$ radiation (graphite monochromator) with a frame time of 10 seconds and a detector distance of $4.0 \mathrm{~cm}$. A randomly oriented region of reciprocal space was surveyed to achieve complete data with a redundancy of 4 . Sections of frames were collected with $0.50^{\circ}$ steps in $\mathrm{w}$ and $\mathrm{f}$ scans. Data to a resolution of $0.84 \AA$ were considered in the reduction. Final cell constants were calculated from the xyz centroids of 2591 strong reflections from the actual data collection after integration (SAINT). The intensity data were corrected for absorption (SADABS). Please refer to Table 5 for additional crystal and refinement information.

Structure solution and refinement

The space group P21/n was determined based on intensity statistics and systematic absences. The structure was solved using Superflip3 and refined (full-matrix-least squares) using the Oxford University Crystals for Windows system. The charge-flipping solution provided most non-hydrogen atoms from the E-map. Full-matrix least squares / difference Fourier cycles were performed, which located the remaining nonhydrogen atoms. All non-hydrogen atoms were refined with anisotropic displacement parameters. The hydrogen atoms were placed in ideal positions and refined with individual relative isotropic displacement 
parameters.

Table S5. Crystal data and structure refinement for Compound 19a.

Empirical formula

Formula weight

Crystal color, shape, size

Temperature

Wavelength

Crystal system, space group

Unit cell dimensions

Volume

Z

Density (Calcd)

Absorption coefficient

$\mathrm{F}(000)$

Data collection

Diffractometer

Theta range for data collection

Index ranges

Reflections collected

Independent reflections

Observed Reflections

\section{C18 H14 C11 N3}

307.78

yellow block fragment, $0.200 \times 0.300 \times 0.300 \mathrm{~mm}^{3}$

$293 \mathrm{~K}$

$1.54178 \AA$

Monoclinic, $\mathrm{P} 21 / \mathrm{n}$

$a=10.1156(4) \AA$

$\mathrm{a}=90^{\circ}$.

$\mathrm{b}=10.7151(4) \AA$

$\mathrm{b}=102.223(2)^{\circ}$.

$\mathrm{c}=14.7073(6) \AA$

$1557.98(11) \AA^{3}$

4

$1.312 \mathrm{Mg} / \mathrm{m}^{3}$

$2.153 \mathrm{~mm}^{-1}$

640

Bruker Apex Kappa Duo, Bruker

4.863 to $72.452^{\circ}$.

$-9<=\mathrm{h}<=11,-9<=\mathrm{k}<=13,-15<=\mathrm{l}<=13$

7948

$2591[\mathrm{R}(\mathrm{int})=0.0209]$

2580

multi-scan

0.52 and 0.65

Direct methods

Full-matrix least-squares on $\mathrm{F}^{2}$

$\mathrm{w}=1 /\left[\mathrm{s}^{2}\left(\mathrm{~F}^{2}\right)+(\mathrm{AP})^{2}+1.18 \mathrm{P}\right]$, where

$\mathrm{P}=\left(\max \left(\mathrm{Fo}^{2}, 0\right)+2 \mathrm{Fc}^{2}\right) / 3, \mathrm{~A}=0.11, \mathrm{~B}=1.18$

$2580 / 93 / 255$

1.002

$\mathrm{R} 1=0.0667, \mathrm{wR} 2=0.1909$

$\mathrm{R} 1=0.0667, \mathrm{wR} 2=0.1909$

0.40 and -0.42 e. $\AA^{-3}$ 
Table S6. Atomic coordinates and equivalent isotropic displacement parameters $\left(\AA^{2}\right)$ for compound 19a. U(eq) is defined as one third of the trace of the orthogonalized $\mathrm{U}^{\mathrm{ij}}$ tensor.

\begin{tabular}{|c|c|c|c|c|}
\hline Atom & $x$ & $y$ & $z$ & $U_{\text {iso/equiv. }}$ \\
\hline $\mathrm{Cl}(1)$ & $0.84333(9)$ & $0.56992(9)$ & $0.56245(7)$ & 0.0839 \\
\hline$C(2)$ & $0.6798(3)$ & $0.6162(3)$ & $0.5628(2)$ & 0.0625 \\
\hline$C(3)$ & $0.6290(4)$ & $0.7225(4)$ & $0.5038(3)$ & 0.0762 \\
\hline $\mathrm{N}(4)$ & $0.6024(3)$ & $0.8022(4)$ & $0.4607(3)$ & 0.0985 \\
\hline $\mathrm{C}(5)$ & $0.6048(3)$ & $0.5575(3)$ & $0.6163(2)$ & 0.0603 \\
\hline$C(6)$ & $0.4728(3)$ & $0.5893(3)$ & $0.6232(2)$ & 0.0588 \\
\hline$C(7)$ & $0.4073(3)$ & $0.5350(2)$ & $0.6856(2)$ & 0.0534 \\
\hline $\mathrm{N}(8)$ & $0.2819(2)$ & $0.5651(2)$ & $0.69591(19)$ & 0.0607 \\
\hline$C(9)$ & $0.2235(3)$ & $0.5204(3)$ & $0.7725(2)$ & 0.0653 \\
\hline$C(10)$ & $0.1042(3)$ & $0.4335(2)$ & $0.7419(2)$ & 0.0516 \\
\hline $\mathrm{C}(11)$ & $0.0986(3)$ & $0.3511(2)$ & $0.6709(2)$ & 0.0509 \\
\hline $\mathrm{C}(12)$ & $-0.0131(3)$ & $0.2706(2)$ & $0.64242(18)$ & 0.0482 \\
\hline$C(13)$ & $-0.1215(3)$ & $0.2763(2)$ & $0.68919(19)$ & 0.0514 \\
\hline $\mathrm{C}(14)$ & $-0.1123(3)$ & $0.3612(3)$ & $0.7641(2)$ & 0.0563 \\
\hline$C(15)$ & $-0.0036(3)$ & $0.4372(3)$ & $0.7892(2)$ & 0.0564 \\
\hline$C(16)$ & $-0.2350(3)$ & $0.1974(3)$ & $0.6598(2)$ & 0.0655 \\
\hline $\mathrm{C}(17)$ & $-0.2390(4)$ & $0.1175(3)$ & $0.5877(3)$ & 0.0731 \\
\hline $\mathrm{C}(18)$ & $-0.1316(4)$ & $0.1099(3)$ & $0.5426(2)$ & 0.0682 \\
\hline $\mathrm{C}(19)$ & $-0.0210(3)$ & $0.1851(3)$ & $0.5678(2)$ & 0.0588 \\
\hline$C(20)$ & $0.2054(3)$ & $0.6582(3)$ & $0.6351(3)$ & 0.0746 \\
\hline $\mathrm{C}(21)$ & $0.4747(3)$ & $0.4368(3)$ & $0.7450(2)$ & 0.0624 \\
\hline $\mathrm{N}(22)$ & $0.5296(3)$ & $0.3587(3)$ & $0.7898(3)$ & 0.0919 \\
\hline
\end{tabular}


Table S7. Bond lengths $[\AA]$ and angles $\left[^{\circ}\right]$ for compound 19a.

\begin{tabular}{|c|c|c|c|}
\hline $\mathrm{Cl}(1)-\mathrm{C}(2)$ & $1.728(3)$ & $\mathrm{C}(12)-\mathrm{C}(13)$ & $1.414(4)$ \\
\hline$C(2)-C(3)$ & $1.458(5)$ & $\mathrm{C}(12)-\mathrm{C}(19)$ & $1.418(4)$ \\
\hline$C(2)-C(5)$ & $1.357(4)$ & $C(13)-C(14)$ & $1.417(4)$ \\
\hline $\mathrm{C}(3)-\mathrm{N}(4)$ & $1.064(4)$ & $\mathrm{C}(13)-\mathrm{C}(16)$ & $1.417(4)$ \\
\hline$C(5)-C(6)$ & $1.403(4)$ & $\mathrm{C}(14)-\mathrm{C}(15)$ & $1.356(4)$ \\
\hline $\mathrm{C}(5)-\mathrm{H}(51)$ & $0.927(17)$ & $\mathrm{C}(14)-\mathrm{H}(141)$ & $0.933(17)$ \\
\hline$C(6)-C(7)$ & $1.370(4)$ & $\mathrm{C}(15)-\mathrm{H}(151)$ & $0.919(17)$ \\
\hline $\mathrm{C}(6)-\mathrm{H}(61)$ & $0.935(17)$ & $C(16)-C(17)$ & $1.358(5)$ \\
\hline $\mathrm{C}(7)-\mathrm{N}(8)$ & $1.348(4)$ & $\mathrm{C}(16)-\mathrm{H}(161)$ & $0.927(18)$ \\
\hline$C(7)-C(21)$ & $1.444(4)$ & $\mathrm{C}(17)-\mathrm{C}(18)$ & $1.391(5)$ \\
\hline $\mathrm{N}(8)-\mathrm{C}(9)$ & $1.459(4)$ & $\mathrm{C}(17)-\mathrm{H}(171)$ & $0.915(18)$ \\
\hline $\mathrm{N}(8)-\mathrm{C}(20)$ & $1.450(4)$ & $\mathrm{C}(18)-\mathrm{C}(19)$ & $1.364(4)$ \\
\hline$C(9)-C(10)$ & $1.516(4)$ & $\mathrm{C}(18)-\mathrm{H}(181)$ & $0.916(18)$ \\
\hline $\mathrm{C}(9)-\mathrm{H}(91)$ & $0.966(18)$ & $\mathrm{C}(19)-\mathrm{H}(191)$ & $0.926(18)$ \\
\hline $\mathrm{C}(9)-\mathrm{H}(92)$ & $0.962(17)$ & $\mathrm{C}(20)-\mathrm{H}(201)$ & $0.965(18)$ \\
\hline $\mathrm{C}(10)-\mathrm{C}(11)$ & $1.359(4)$ & $\mathrm{C}(20)-\mathrm{H}(202)$ & $0.953(18)$ \\
\hline$C(10)-C(15)$ & $1.412(4)$ & $\mathrm{C}(20)-\mathrm{H}(203)$ & $0.951(18)$ \\
\hline $\mathrm{C}(11)-\mathrm{C}(12)$ & $1.413(4)$ & $\mathrm{C}(21)-\mathrm{N}(22)$ & $1.134(4)$ \\
\hline $\mathrm{C}(11)-\mathrm{H}(111)$ & $0.912(17)$ & & \\
\hline $\mathrm{Cl}(1)-\mathrm{C}(2)-\mathrm{C}(3)$ & $116.0(2)$ & $\mathrm{C}(13)-\mathrm{C}(12)-\mathrm{C}(19)$ & $118.8(3)$ \\
\hline $\mathrm{Cl}(1)-\mathrm{C}(2)-\mathrm{C}(5)$ & $121.5(3)$ & $\mathrm{C}(12)-\mathrm{C}(13)-\mathrm{C}(14)$ & $118.4(2)$ \\
\hline$C(3)-C(2)-C(5)$ & $122.5(3)$ & $\mathrm{C}(12)-\mathrm{C}(13)-\mathrm{C}(16)$ & $119.2(3)$ \\
\hline$C(2)-C(3)-N(4)$ & $174.2(4)$ & $\mathrm{C}(14)-\mathrm{C}(13)-\mathrm{C}(16)$ & $122.4(3)$ \\
\hline$C(2)-C(5)-C(6)$ & $126.0(3)$ & $\mathrm{C}(13)-\mathrm{C}(14)-\mathrm{C}(15)$ & $120.9(3)$ \\
\hline $\mathrm{C}(2)-\mathrm{C}(5)-\mathrm{H}(51)$ & $118.2(12)$ & $\mathrm{C}(13)-\mathrm{C}(14)-\mathrm{H}(141)$ & $119.5(12)$ \\
\hline $\mathrm{C}(6)-\mathrm{C}(5)-\mathrm{H}(51)$ & $115.8(12)$ & $\mathrm{C}(15)-\mathrm{C}(14)-\mathrm{H}(141)$ & $119.6(12)$ \\
\hline$C(5)-C(6)-C(7)$ & $123.3(3)$ & $\mathrm{C}(10)-\mathrm{C}(15)-\mathrm{C}(14)$ & $121.0(3)$ \\
\hline $\mathrm{C}(5)-\mathrm{C}(6)-\mathrm{H}(61)$ & $114.9(12)$ & $\mathrm{C}(10)-\mathrm{C}(15)-\mathrm{H}(151)$ & $119.1(12)$ \\
\hline
\end{tabular}




\begin{tabular}{|c|c|c|c|}
\hline $\mathrm{C}(7)-\mathrm{C}(6)-\mathrm{H}(61)$ & $121.8(12)$ & $\mathrm{C}(14)-\mathrm{C}(15)-\mathrm{H}(151)$ & $119.9(12)$ \\
\hline$C(6)-C(7)-N(8)$ & $125.0(3)$ & $C(13)-C(16)-C(17)$ & $120.2(3)$ \\
\hline$C(6)-C(7)-C(21)$ & $118.8(3)$ & $\mathrm{C}(13)-\mathrm{C}(16)-\mathrm{H}(161)$ & $119.1(12)$ \\
\hline $\mathrm{N}(8)-\mathrm{C}(7)-\mathrm{C}(21)$ & $116.2(3)$ & $C(17)-C(16)-H(161)$ & $120.8(12)$ \\
\hline $\mathrm{C}(7)-\mathrm{N}(8)-\mathrm{C}(9)$ & $123.6(3)$ & $\mathrm{C}(16)-\mathrm{C}(17)-\mathrm{C}(18)$ & $120.8(3)$ \\
\hline $\mathrm{C}(7)-\mathrm{N}(8)-\mathrm{C}(20)$ & $119.2(3)$ & $\mathrm{C}(16)-\mathrm{C}(17)-\mathrm{H}(171)$ & $119.3(12)$ \\
\hline $\mathrm{C}(9)-\mathrm{N}(8)-\mathrm{C}(20)$ & $116.9(3)$ & $\mathrm{C}(18)-\mathrm{C}(17)-\mathrm{H}(171)$ & $119.9(12)$ \\
\hline $\mathrm{N}(8)-\mathrm{C}(9)-\mathrm{C}(10)$ & $113.6(3)$ & $\mathrm{C}(17)-\mathrm{C}(18)-\mathrm{C}(19)$ & $120.9(3)$ \\
\hline $\mathrm{N}(8)-\mathrm{C}(9)-\mathrm{H}(91)$ & $108.9(13)$ & $\mathrm{C}(17)-\mathrm{C}(18)-\mathrm{H}(181)$ & $120.6(12)$ \\
\hline $\mathrm{C}(10)-\mathrm{C}(9)-\mathrm{H}(91)$ & $107.8(13)$ & $\mathrm{C}(19)-\mathrm{C}(18)-\mathrm{H}(181)$ & $118.5(12)$ \\
\hline N(8)-C(9)-H(92) & $109.0(13)$ & $C(12)-C(19)-C(18)$ & $120.1(3)$ \\
\hline $\mathrm{C}(10)-\mathrm{C}(9)-\mathrm{H}(92)$ & $107.8(13)$ & $\mathrm{C}(12)-\mathrm{C}(19)-\mathrm{H}(191)$ & $120.6(12)$ \\
\hline H(91)-C(9)-H(92) & $109.8(15)$ & $\mathrm{C}(18)-\mathrm{C}(19)-\mathrm{H}(191)$ & $119.4(12)$ \\
\hline$C(9)-C(10)-C(11)$ & $121.9(3)$ & $\mathrm{N}(8)-\mathrm{C}(20)-\mathrm{H}(201)$ & $110.6(15)$ \\
\hline$C(9)-C(10)-C(15)$ & 119.1(3) & $\mathrm{N}(8)-\mathrm{C}(20)-\mathrm{H}(202)$ & $109.8(15)$ \\
\hline$C(11)-C(10)-C(15)$ & 119.1(3) & $\mathrm{H}(201)-\mathrm{C}(20)-\mathrm{H}(202)$ & $109.7(16)$ \\
\hline$C(10)-C(11)-C(12)$ & $121.7(3)$ & $\mathrm{N}(8)-\mathrm{C}(20)-\mathrm{H}(203)$ & $110.6(15)$ \\
\hline $\mathrm{C}(10)-\mathrm{C}(11)-\mathrm{H}(111)$ & $120.1(11)$ & $\mathrm{H}(201)-\mathrm{C}(20)-\mathrm{H}(203)$ & $107.3(16)$ \\
\hline $\mathrm{C}(12)-\mathrm{C}(11)-\mathrm{H}(111)$ & $118.2(11)$ & $\mathrm{H}(202)-\mathrm{C}(20)-\mathrm{H}(203)$ & $108.7(16)$ \\
\hline$C(11)-C(12)-C(13)$ & $118.9(2)$ & $\mathrm{C}(7)-\mathrm{C}(21)-\mathrm{N}(22)$ & $178.0(4)$ \\
\hline$C(11)-C(12)-C(19)$ & $122.3(3)$ & & \\
\hline
\end{tabular}


Table S8. Anisotropic displacement parameters $\left(\AA^{2}\right)$ for compound 19a. The anisotropic displacement factor exponent takes the form: $-2 \pi^{2}\left[h^{2} a^{* 2} U^{11}+\ldots+2 h k a^{*} b^{*} U^{12}\right]$.

\begin{tabular}{|c|c|c|c|c|c|c|}
\hline Label & $\mathrm{U}_{11}$ & $\mathrm{U}_{22}$ & $\mathrm{U}_{33}$ & $\mathrm{U}_{23}$ & $\mathrm{U}_{13}$ & $\mathrm{U}_{12}$ \\
\hline $\mathrm{C} 11$ & $0.0739(6)$ & $0.0904(6)$ & $0.0911(7)$ & $0.0183(5)$ & $0.0259(5)$ & $0.0054(4)$ \\
\hline $\mathrm{C} 2$ & $0.0711(19)$ & $0.0621(16)$ & $0.0548(18)$ & $0.0034(14)$ & $0.0148(15)$ & $-0.0090(14)$ \\
\hline $\mathrm{C} 3$ & $0.077(2)$ & $0.082(2)$ & $0.077(2)$ & $0.0035(19)$ & $0.0347(19)$ & $-0.0092(18)$ \\
\hline N4 & $0.086(2)$ & $0.105(2)$ & $0.110(3)$ & $0.053(2)$ & $0.0324(19)$ & $0.0244(18)$ \\
\hline $\mathrm{C} 5$ & $0.0686(19)$ & $0.0537(15)$ & $0.0569(18)$ & $0.0022(13)$ & $0.0096(14)$ & $-0.0040(13)$ \\
\hline C6 & $0.0554(17)$ & $0.0566(15)$ & $0.0640(19)$ & $-0.0020(13)$ & $0.0118(14)$ & $-0.0058(12)$ \\
\hline $\mathrm{C} 7$ & $0.0498(15)$ & $0.0508(13)$ & $0.0589(17)$ & $-0.0077(12)$ & $0.0099(13)$ & $-0.0104(11)$ \\
\hline N8 & $0.0499(14)$ & $0.0591(14)$ & $0.0740(18)$ & $-0.0005(11)$ & $0.0153(12)$ & $-0.0046(10)$ \\
\hline C9 & $0.0545(17)$ & $0.077(2)$ & $0.066(2)$ & $-0.0153(16)$ & $0.0169(15)$ & $-0.0050(14)$ \\
\hline $\mathrm{C} 10$ & $0.0462(14)$ & $0.0557(14)$ & $0.0527(17)$ & $0.0003(12)$ & $0.0101(12)$ & $0.0042(11)$ \\
\hline $\mathrm{C} 11$ & $0.0433(13)$ & $0.0565(14)$ & $0.0558(17)$ & $0.0035(12)$ & $0.0172(12)$ & $0.0050(10)$ \\
\hline $\mathrm{C} 12$ & $0.0489(14)$ & $0.0443(12)$ & $0.0515(16)$ & $0.0080(11)$ & $0.0111(12)$ & $0.0074(10)$ \\
\hline $\mathrm{C} 13$ & $0.0491(14)$ & $0.0485(13)$ & $0.0572(17)$ & $0.0111(11)$ & $0.0126(12)$ & $0.0048(10)$ \\
\hline $\mathrm{C} 14$ & $0.0528(16)$ & $0.0591(15)$ & $0.0637(18)$ & $0.0045(13)$ & $0.0271(14)$ & $0.0062(12)$ \\
\hline $\mathrm{C} 15$ & $0.0563(17)$ & $0.0603(16)$ & $0.0564(17)$ & $-0.0043(13)$ & $0.0206(14)$ & $0.0063(12)$ \\
\hline $\mathrm{C} 16$ & $0.0556(17)$ & $0.0623(16)$ & $0.081(2)$ & $0.0086(16)$ & $0.0192(15)$ & $-0.0038(13)$ \\
\hline $\mathrm{C} 17$ & $0.067(2)$ & $0.0620(17)$ & $0.086(2)$ & $0.0016(17)$ & $0.0068(18)$ & $-0.0148(14)$ \\
\hline $\mathrm{C} 18$ & $0.081(2)$ & $0.0532(16)$ & $0.067(2)$ & $-0.0060(14)$ & $0.0075(17)$ & $-0.0024(14)$ \\
\hline C19 & $0.0665(18)$ & $0.0537(14)$ & $0.0583(18)$ & $0.0010(13)$ & $0.0175(14)$ & $0.0076(12)$ \\
\hline $\mathrm{C} 20$ & $0.0612(18)$ & $0.0588(17)$ & $0.101(3)$ & $0.0002(17)$ & $0.0110(18)$ & $0.0011(14)$ \\
\hline $\mathrm{C} 21$ & $0.0477(16)$ & $0.0686(18)$ & $0.074(2)$ & $0.0037(15)$ & $0.0207(15)$ & $-0.0060(13)$ \\
\hline N22 & $0.0738(19)$ & $0.095(2)$ & $0.110(3)$ & $0.037(2)$ & $0.0270(18)$ & $0.0089(17)$ \\
\hline
\end{tabular}


Table S9. Hydrogen coordinates and displacement parameters $\left(\AA^{2}\right)$ for compound 19a.

\begin{tabular}{|c|c|c|c|c|}
\hline Label & $x$ & $y$ & $z$ & $\mathrm{U}_{\text {iso/equiv }}$ \\
\hline H51 & $0.6433(18)$ & $0.4909(14)$ & $0.6527(10)$ & $0.0721(19)$ \\
\hline H61 & $0.4331(18)$ & $0.6531(14)$ & $0.5833(10)$ & $0.0710(19)$ \\
\hline H91 & $0.193(2)$ & $0.5913(18)$ & $0.8029(16)$ & $0.0775(19)$ \\
\hline H92 & $0.292(2)$ & $0.476(2)$ & $0.8159(15)$ & $0.0781(19)$ \\
\hline H111 & $0.1678(17)$ & $0.3469(13)$ & $0.6401(10)$ & $0.0606(19)$ \\
\hline H141 & $-0.1819(17)$ & $0.3646(14)$ & $0.7968(11)$ & $0.0674(19)$ \\
\hline H151 & $-0.0003(17)$ & $0.4937(14)$ & $0.8366(10)$ & $0.0679(19)$ \\
\hline H161 & $-0.3061(17)$ & $0.2013(14)$ & $0.6905(11)$ & $0.0786(19)$ \\
\hline H171 & $-0.3140(18)$ & $0.0687(14)$ & $0.5683(11)$ & $0.0884(19)$ \\
\hline H181 & $-0.1327(18)$ & $0.0529(14)$ & $0.4960(11)$ & $0.0820(19)$ \\
\hline H191 & $0.0475(17)$ & $0.1808(14)$ & $0.5351(11)$ & $0.0701(19)$ \\
\hline H201 & $0.115(2)$ & $0.665(3)$ & $0.646(2)$ & $0.112(2)$ \\
\hline H202 & $0.250(3)$ & $0.7369(19)$ & $0.645(2)$ & $0.1115(19)$ \\
\hline H203 & $0.197(3)$ & $0.635(2)$ & $0.5717(14)$ & $0.112(2)$ \\
\hline
\end{tabular}




\section{References}

1. Kazakov, P. V.; Golosov, S. N. Identification of impurities in the production of terbinafine hydrochloride. Pharm. Chem. J. 2006, 40, 452-454.

2. Lee, H.-Y.; Jung, Y.; Yoon, Y.; Kim, B. G.; Kim, Y. Angularly Fused Triquinanes from Linear Substrates through Trimethylenemethane Diyl [2 + 3] Cycloaddition Reaction. Org. Lett. 2010, 12, 2672-2674.

3. Coutant, E. P.; Hervin, V.; Gagnot, G.; Ford, C.; Baatallah, R.; Janin, Y. L. Unnatural $\alpha$-amino ethyl esters from diethyl malonate or ethyl $\beta$-bromo- $\alpha$-hydroxyiminocarboxylate. Beilstein J. Org. Chem. 2018, 14, 2853-2860.

4. Jones, D. T.; Artman III, G. D.; Williams, R. M. Coupling of activated esters to gramines in the presence of ethyl propiolate under mild conditions. Tetrahedron Lett. 2007, 48, 1291-1294.

5. Pirrung, M. C. Appendix 3: Recipes for TLC stains. The Synthetic Organic Chemist's Companion; Wiley: Hoboken, 2007; p 171.

6. Godha, A. K.; Thiruvengadam, J.; Abhilash, V.; Balgi, P.; Narayanareddy, A.V.; Vignesh, K.; Gadakh, A.V.; Sathiyanarayanan, A. M.; Ganesh, S. Environmentally benign nucleophilic substitution reaction of arylalkyl halides in water using CTAB as the inverse phase transfer catalyst. New J. Chem. 2019, 43, 1604116045.

7. Böhme, T. M.; Keim, C.; Kreutzmann, K.; Linder, M.; Dingerman, T.; Dannhardt, G.; Mutschler, E. Lambrecht, G. Structure-Activity Relationships of Dimethindene Derivatives as New $\mathrm{M}_{2}$-Selective Muscarinic Receptor Antagonists. J. Med. Chem. 2003, 46, 856-867.

8. Ghosh, S.; Jana, C. K. Metal free biomimetic deaminative direct C-C coupling of unprotected primary amines with active methylene compounds. Org. Biomol. Chem. 2019, 17, 10153-10157.

9. SpinWorks 4.2.0, Copyright 2015, Kirk Marat University of Manitoba.

10. a) Hobgood, T. T.; Goldstein, J. H. Proton magnetic resonance studies of butadiene-1,3 and some of its derivatives. J. Mol. Spectr. 1964, 12, 76-68.; b) Bothner-By, A. A.; Harris, R. K. Nuclear Magnetic Resonance Studies of 1,3-Butadienes. I. The Spectra of Halogenated Butadienes. J. Am. Chem. Soc. 1965, 87, 3445-3450.; c) Bothner-By, A. A.; Harris, R. K. Nuclear Magnetic Resonance Studies of 1,3Butadienes. II. The Relation of $(\mathrm{H}, \mathrm{H})$ Coupling Constants to Conformation ${ }^{1}$. J. Am. Chem. Soc. 1965, 87, 3451-3455.; d) Botta, D.; Mantica, E.; Castellani, L.; Dotelli, G.; Zetta, L. ${ }^{1} \mathrm{H}$ and study of some polychlorobuta-1,3-diene ${ }^{13} \mathrm{C}$ NMR derivatives. Magn. Reson. Chem. 1998, 36, 885-891. 


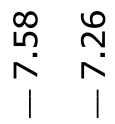

\section{$\mathrm{CDCl}_{3}, 600 \mathrm{MHz}$}<smiles>Clc1ccc(Cl)c2nsnc12</smiles>

$5 a$

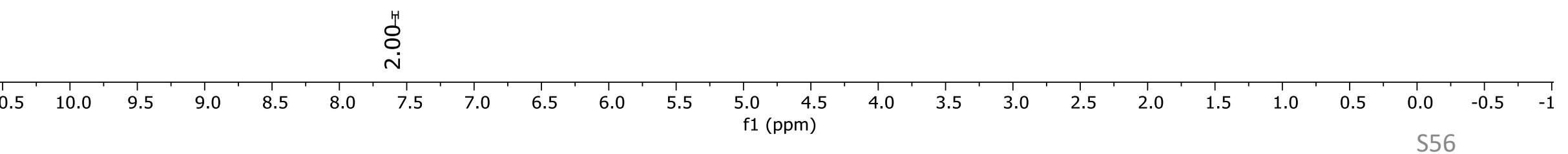




$$
41
$$




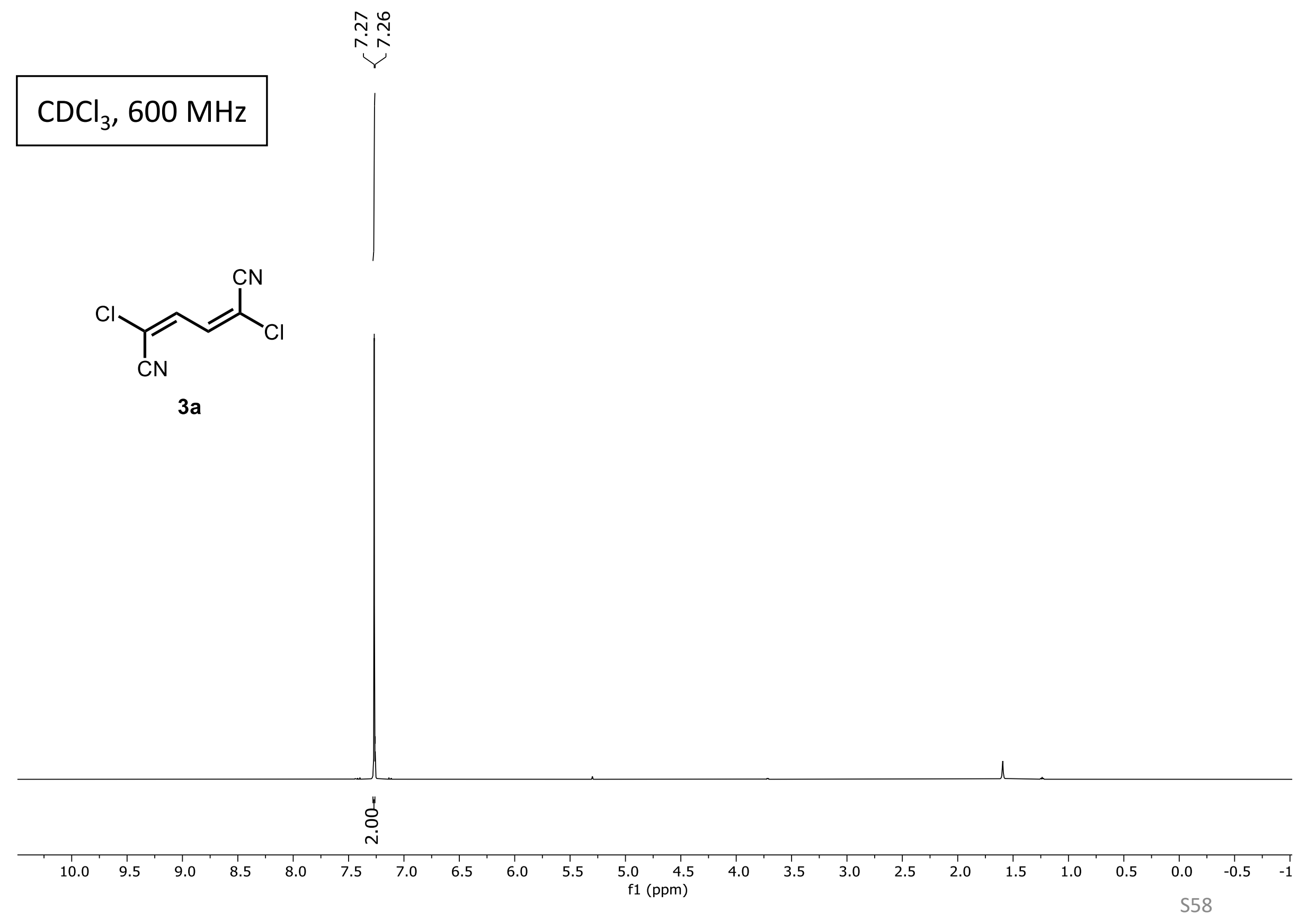




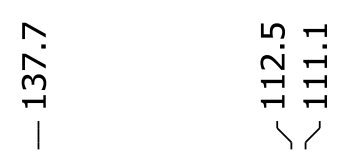

\section{$\mathrm{CDCl}_{3}, 151 \mathrm{MHz}$}<smiles>N#C/C(Cl)=C\C=C(\Cl)C#N</smiles>

$3 a$$$
20
$$$$
\begin{array}{lrrrr}
10 & 200 & 190 & 180 & 170
\end{array}
$$$$
160 \quad 150 \quad 140
$$$$
130 \quad 120
$$$$
10 \quad 100
$$$$
\begin{aligned}
& 100 \\
& (p p m)
\end{aligned}
$$

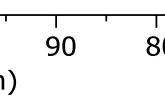

$\begin{array}{ll}1 & 1 \\ 70 & 60\end{array}$

$50 \quad 40$

40

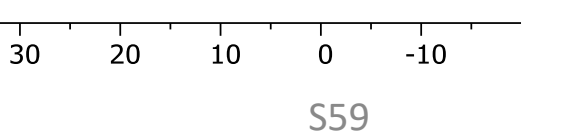


ํำ

\section{$\mathrm{CDCl}_{3}, 600 \mathrm{MHz}$}<smiles>N#C/C(Br)=C\C=C(Br)Br</smiles>

$3 b$

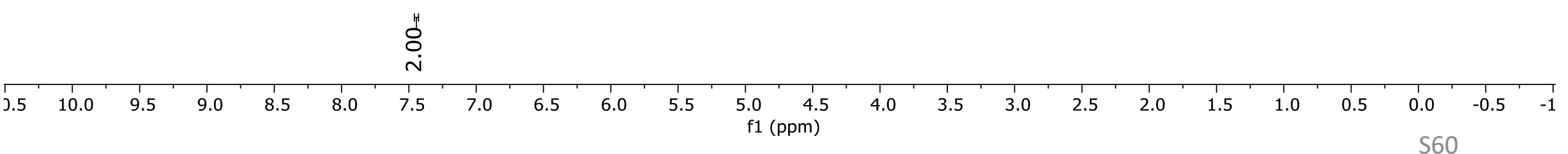




\section{$\mathrm{CDCl}_{3}, 151 \mathrm{MHz}$}

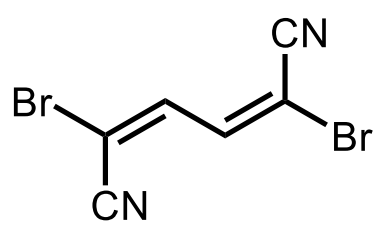

$3 b$

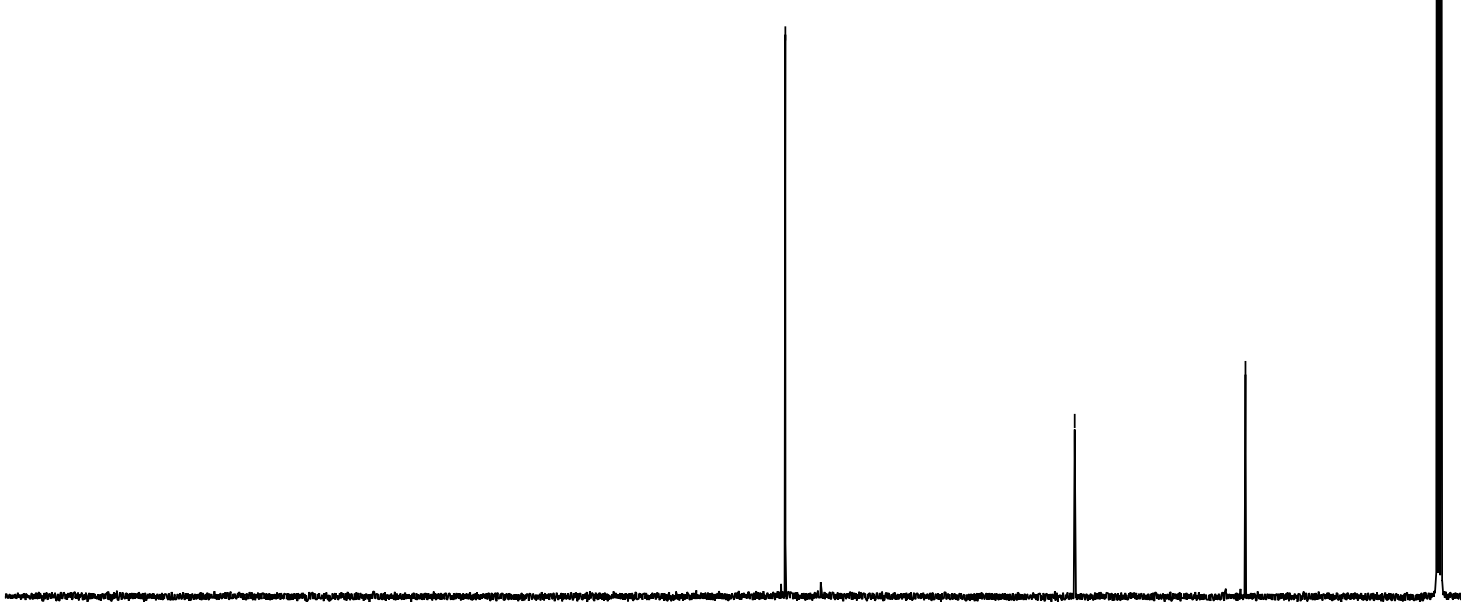$$
210 \quad 200
$$$$
\begin{array}{rrrr}
200 & 190 & 180 & 170
\end{array}
$$$$
160
$$$$
150 \quad 140
$$$$
130 \quad 120
$$$$
110 \quad 100
$$$$
\text { f1 (ppm) }
$$

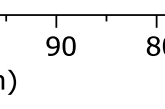

$70 \quad 60$

$60 \quad 50$

40

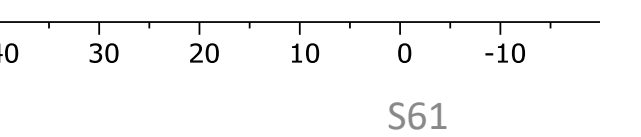




\section{$\mathrm{CDCl}_{3}, 600 \mathrm{MHz}$}

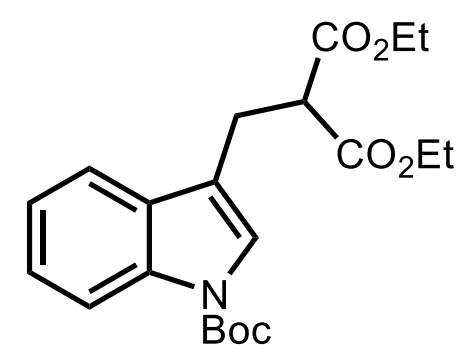

S7

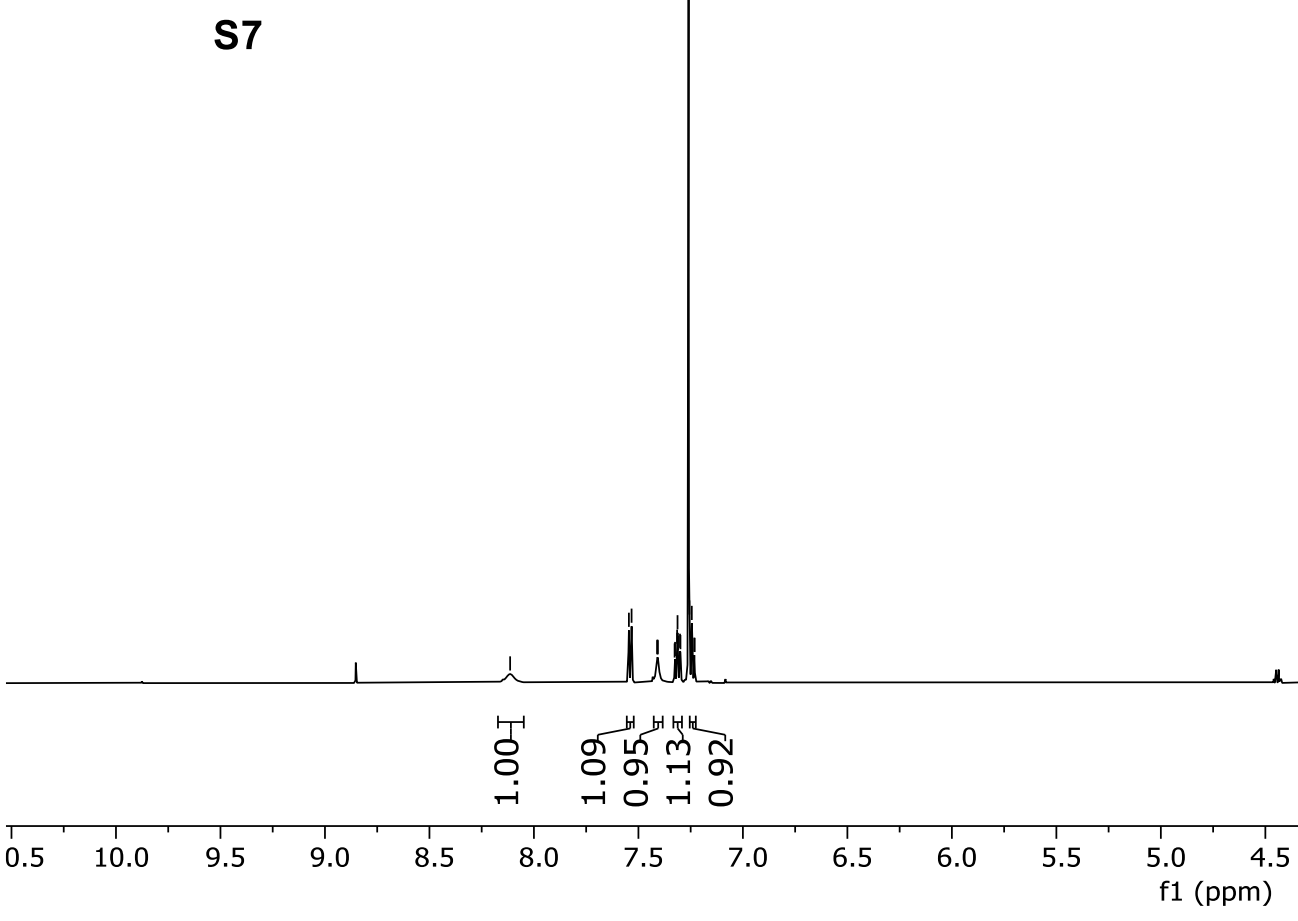

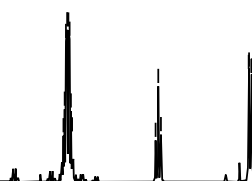

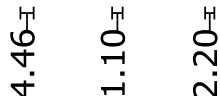



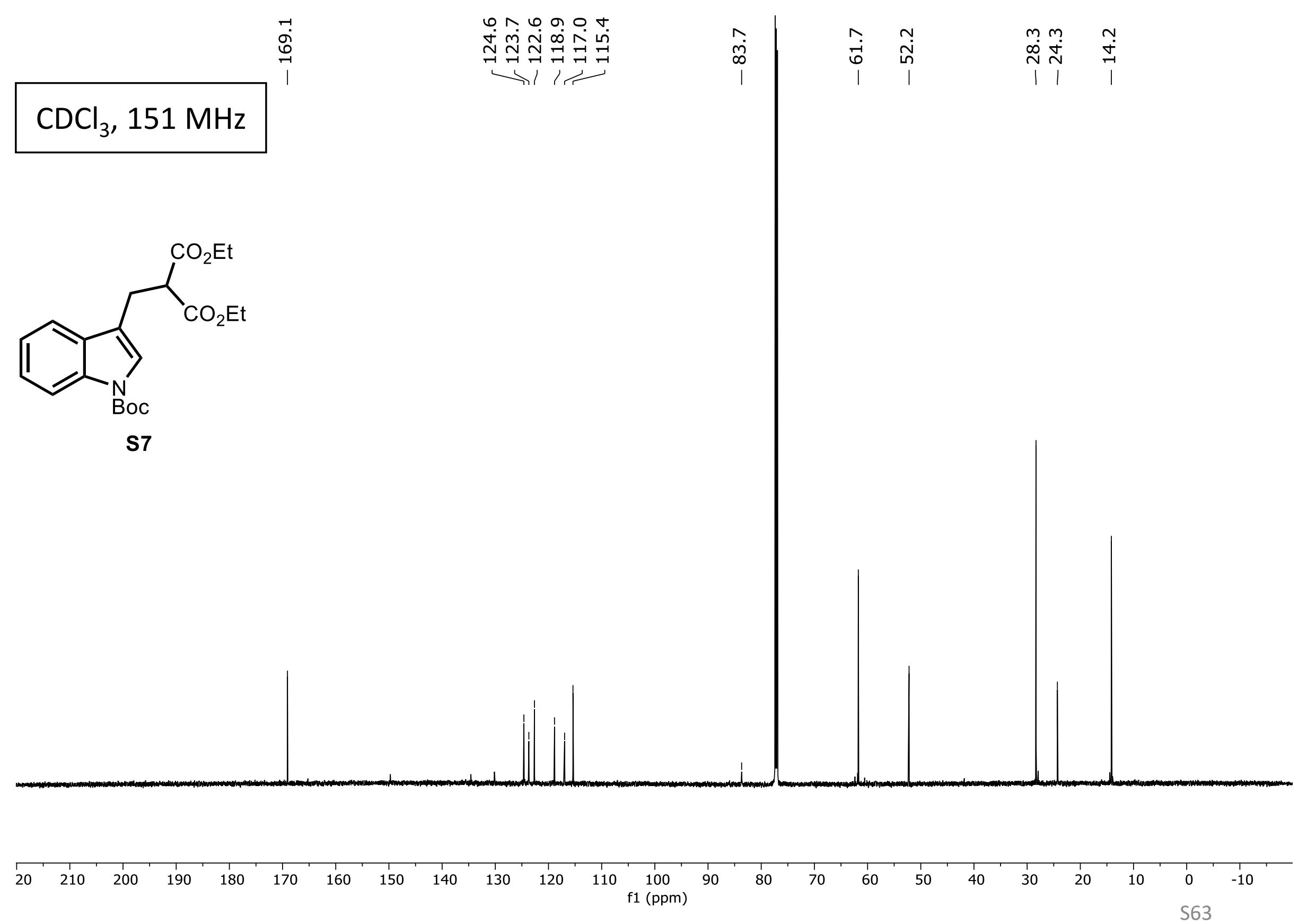


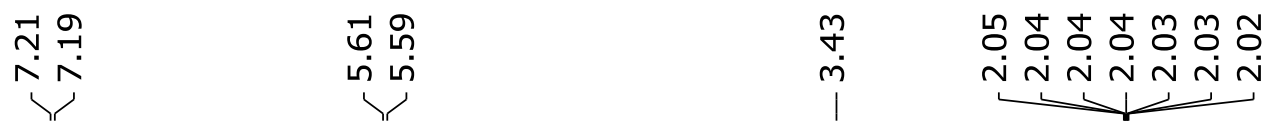

\section{$\mathrm{CDCl}_{3}, 600 \mathrm{MHz}$}
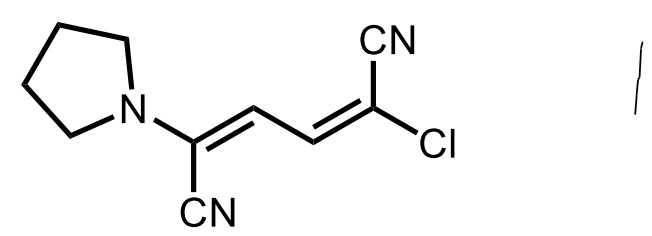

$7 a$

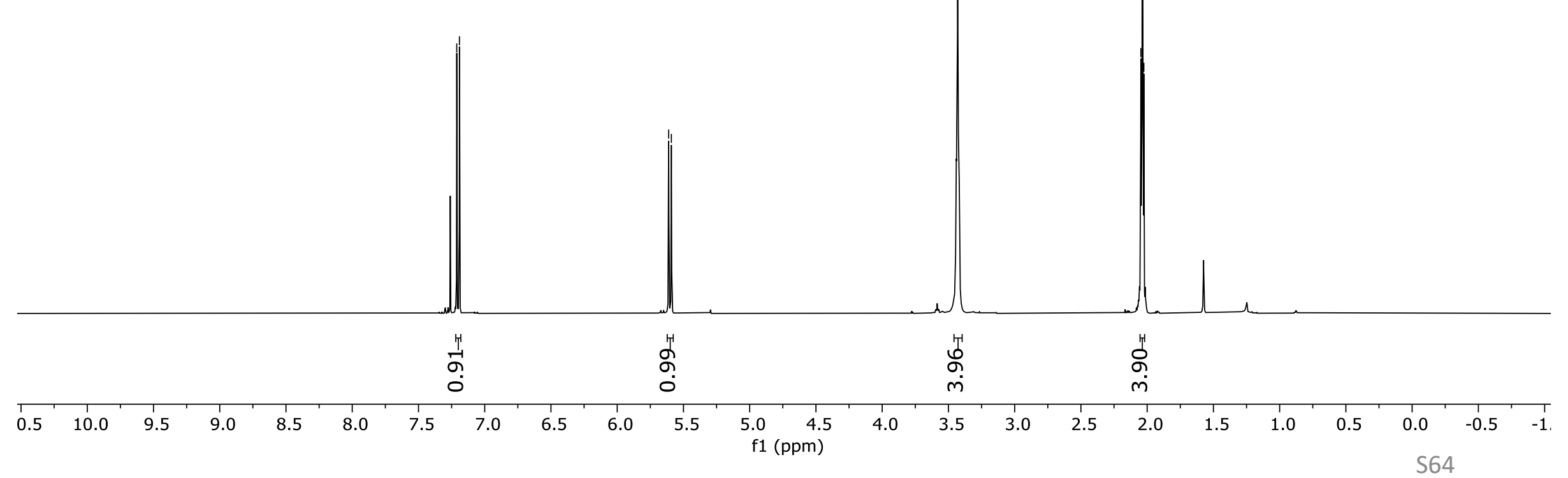




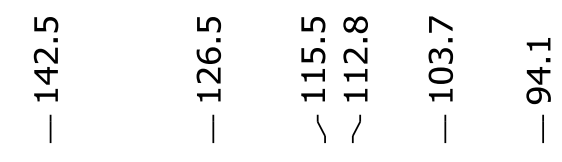

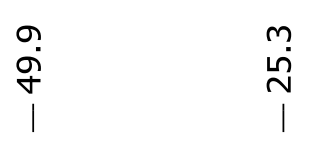

\section{$\mathrm{CDCl}_{3}, 151 \mathrm{MHz}$}

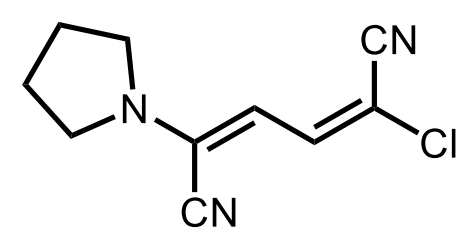

$7 a$
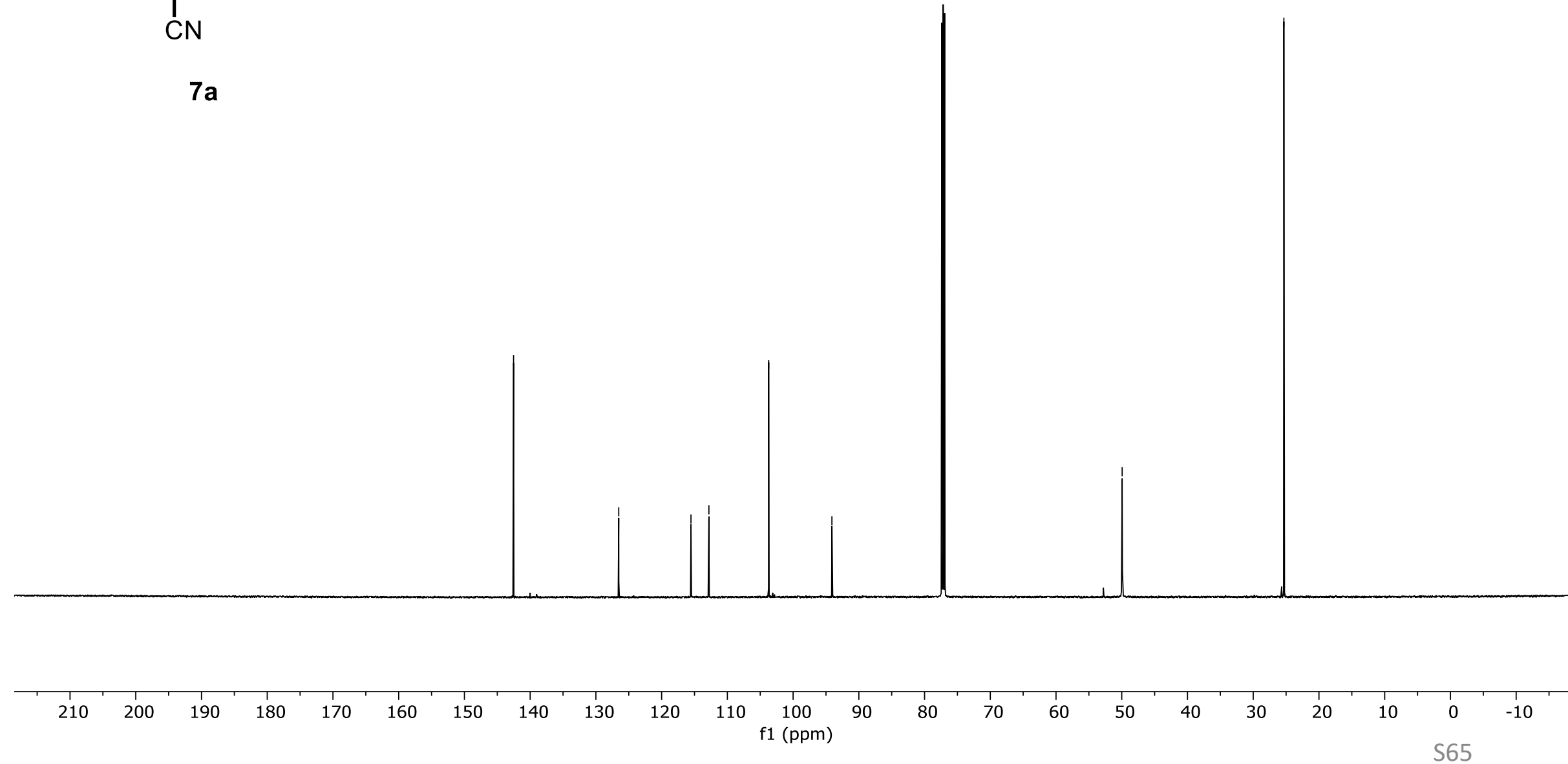


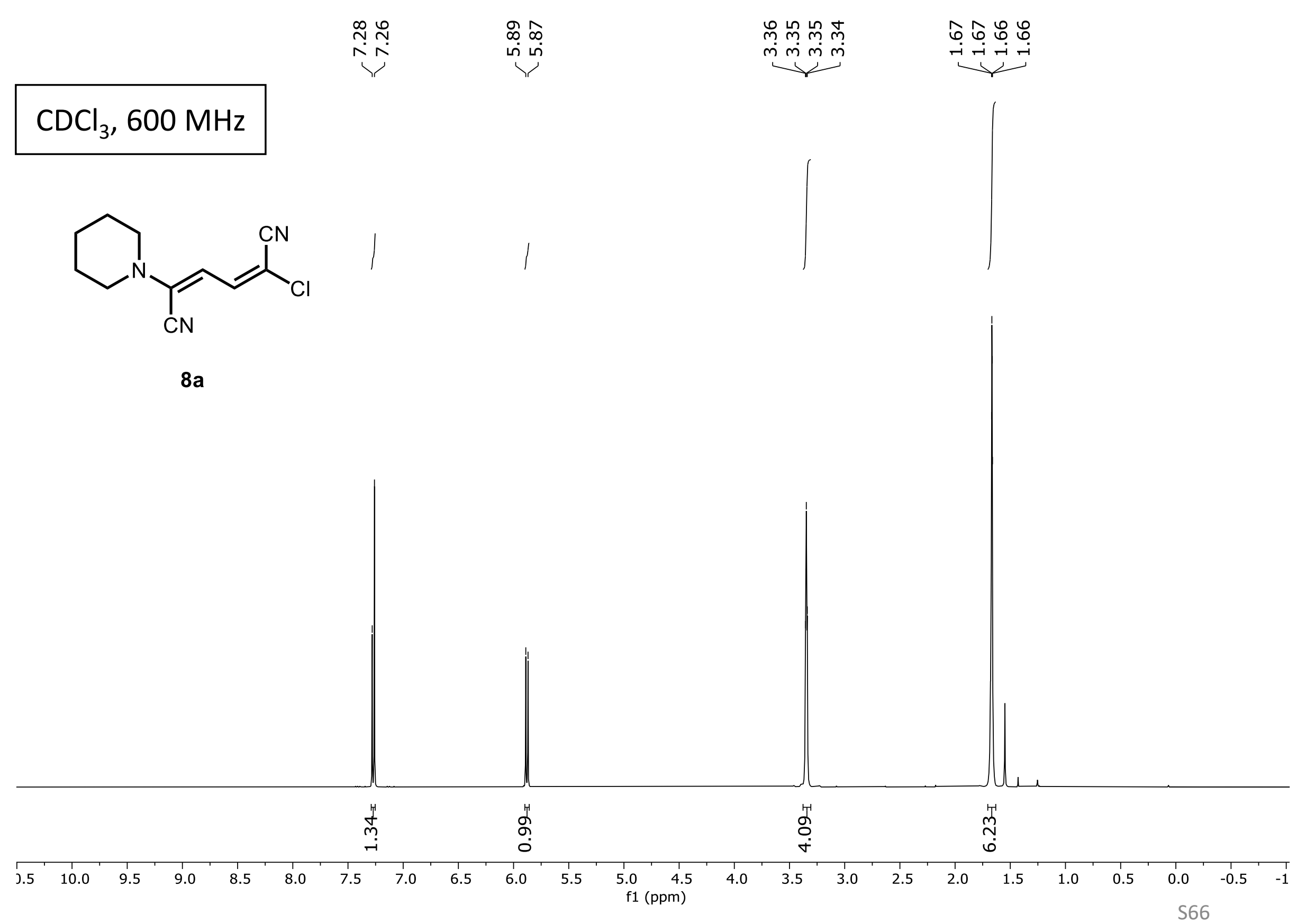




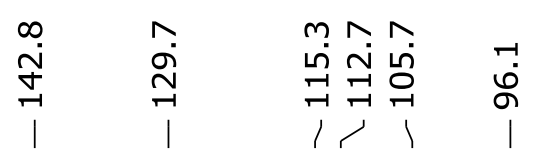

ค่

N

$\stackrel{\sim}{\sim} \stackrel{\infty}{\sim}$

\section{$\mathrm{CDCl}_{3}, 151 \mathrm{MHz}$}<smiles>N#C/C(Cl)=C\C=C(/C#N)N1CCCCC1</smiles>

$8 a$
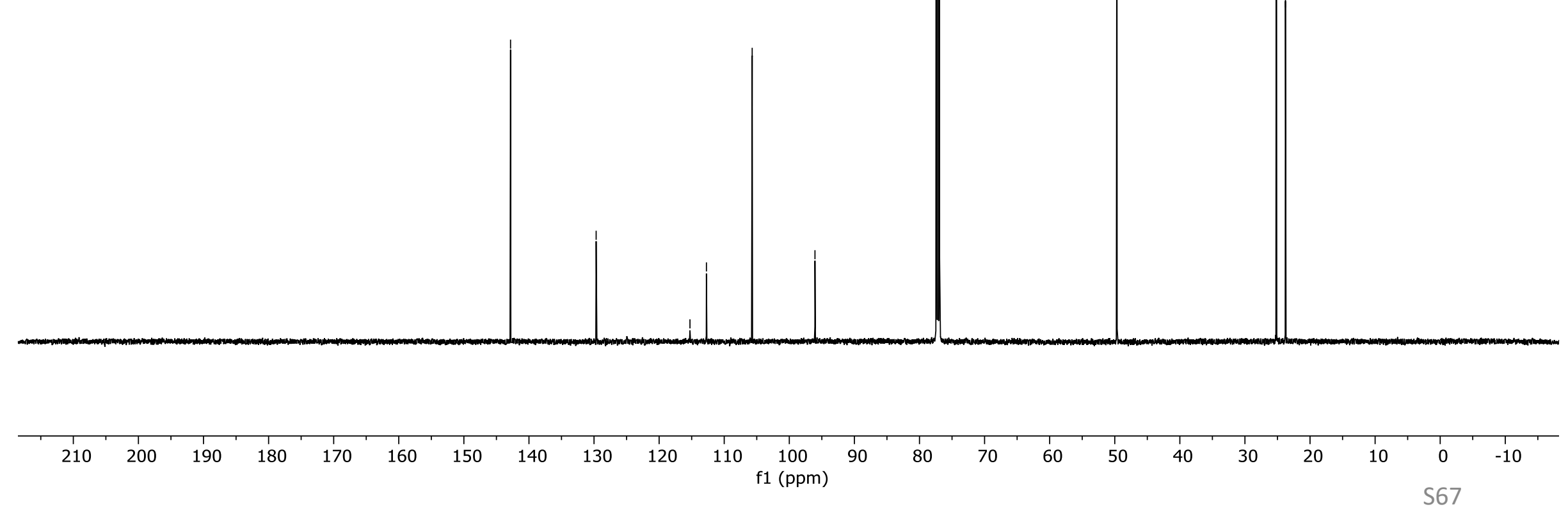
$\stackrel{\text { ำ }}{\stackrel{4}{N}}$

in

m

守守 f

$m \dot{m}$

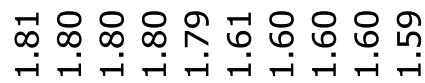

r
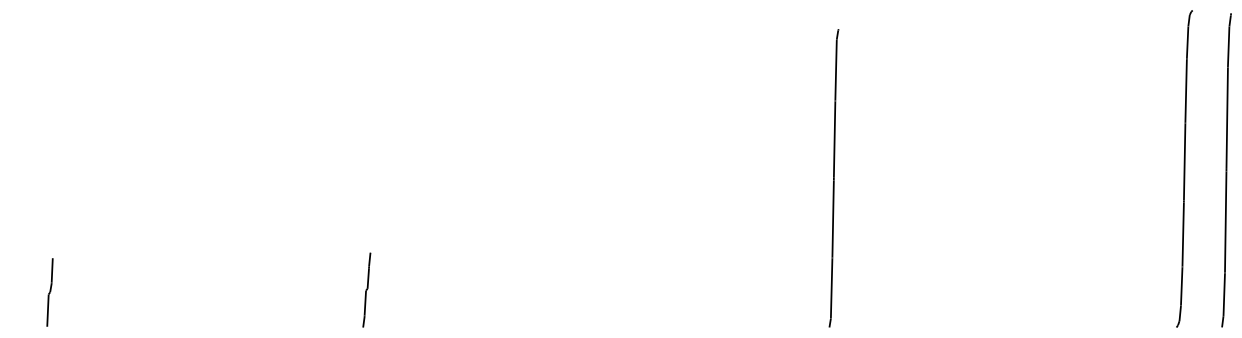

\section{$\mathrm{CDCl}_{3}, 600 \mathrm{MHz}$}
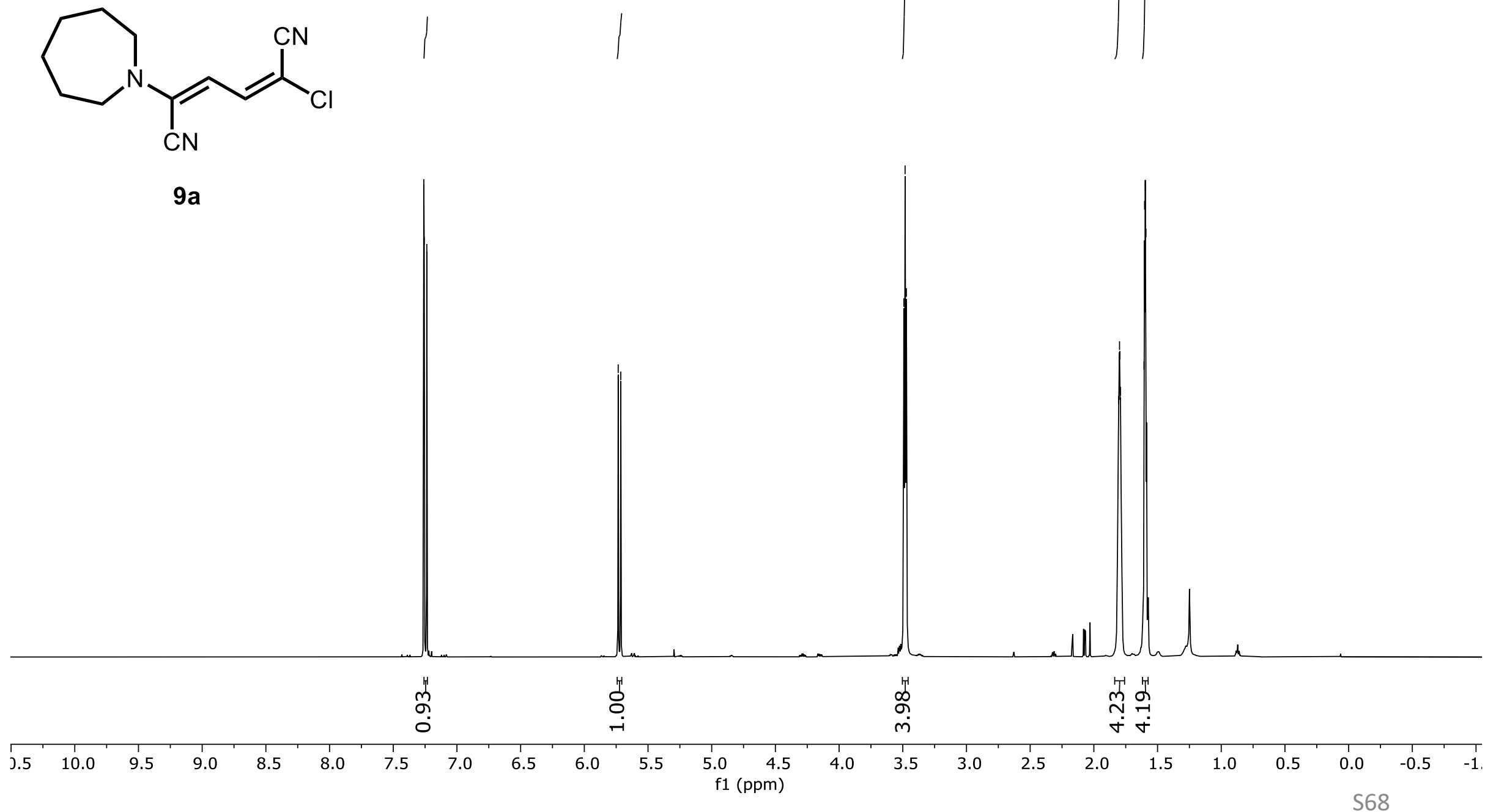


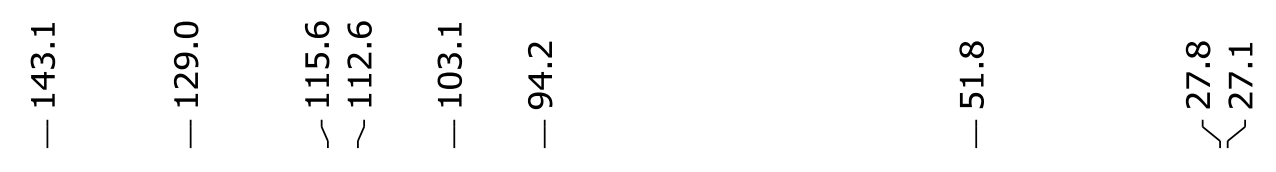

\section{$\mathrm{CDCl}_{3}, 151 \mathrm{MHz}$}

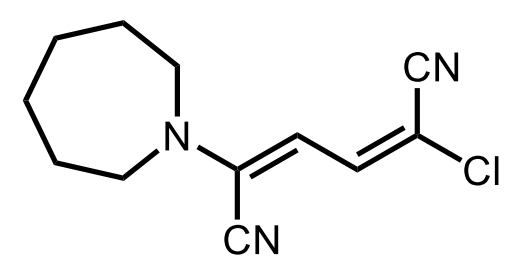

$9 a$
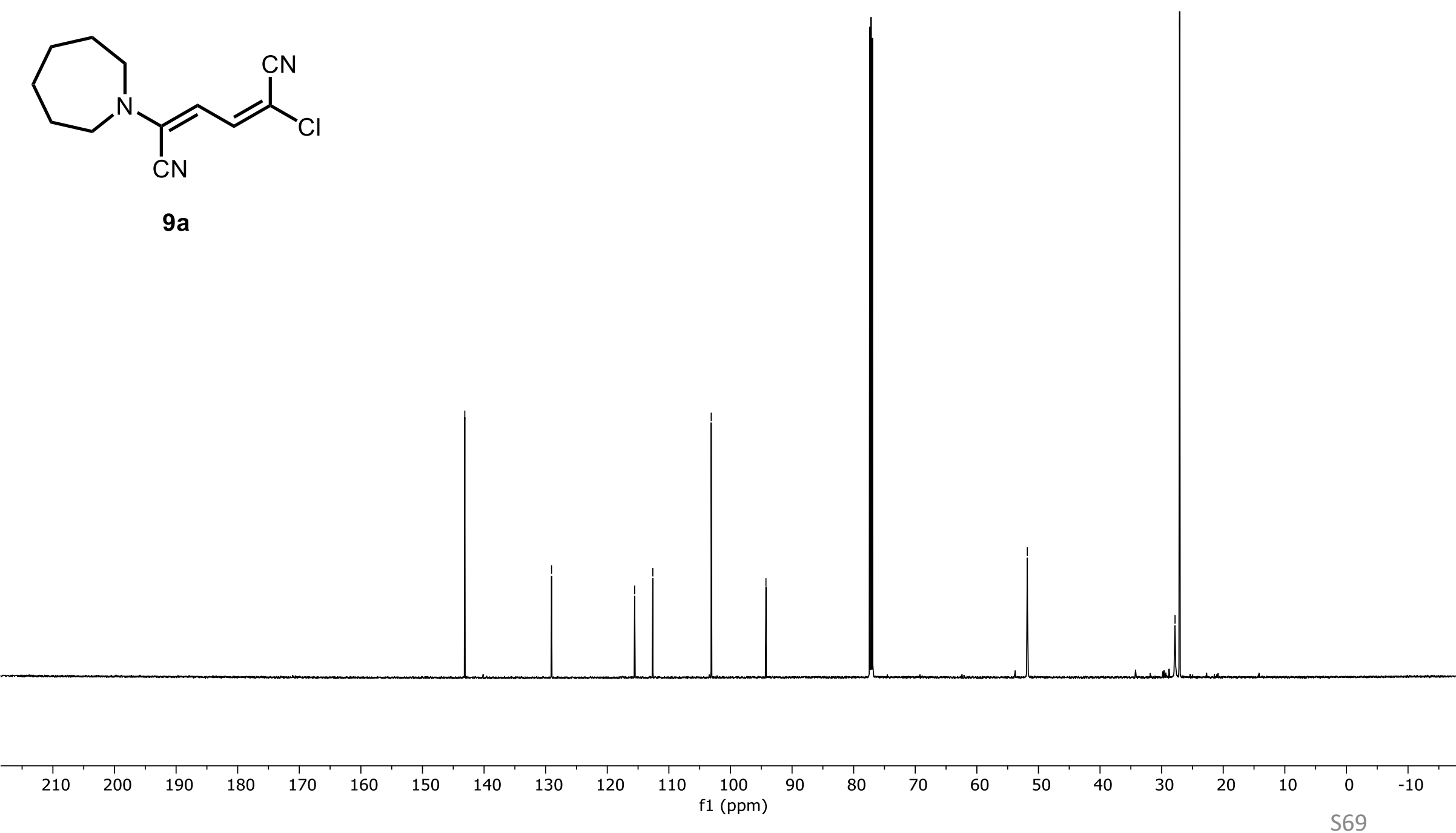


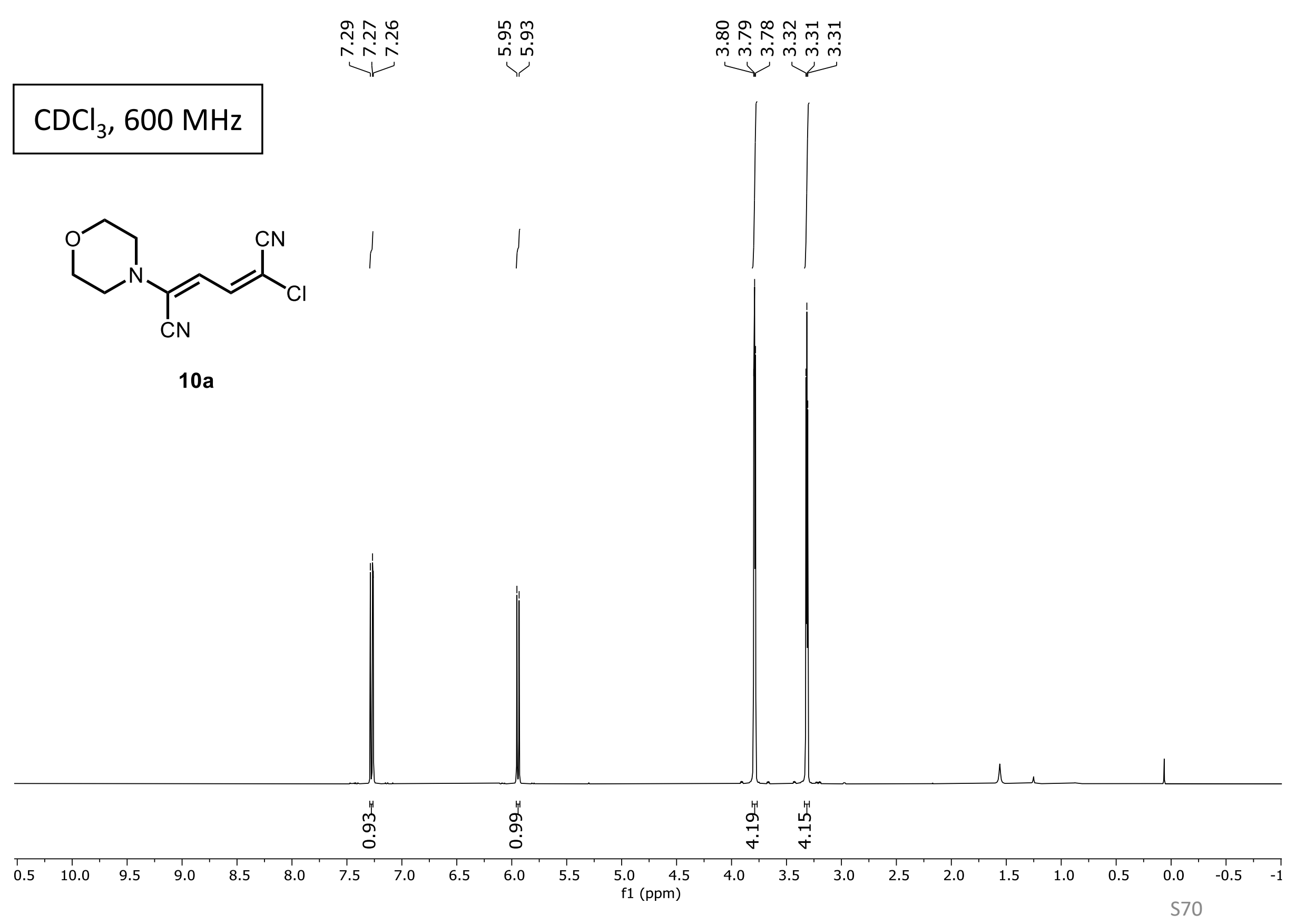




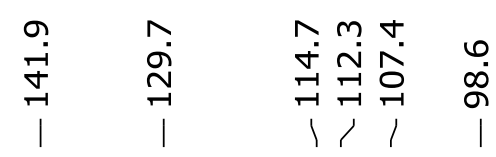

$\begin{array}{ll}\infty & m \\ \text { n் } & \infty \\ 0 & \text { । }\end{array}$

\section{$\mathrm{CDCl}_{3}, 151 \mathrm{MHz}$}

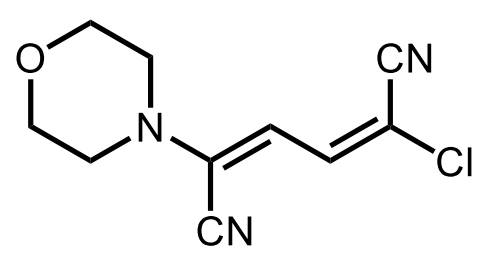

$10 a$

$$
\longrightarrow
$$
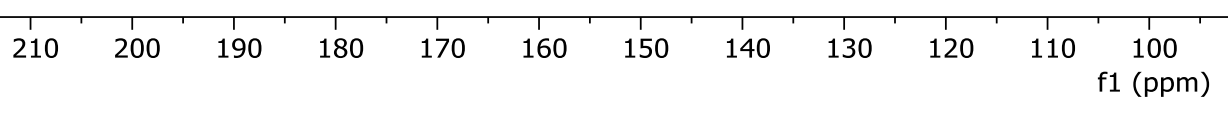
f1 (ppm)

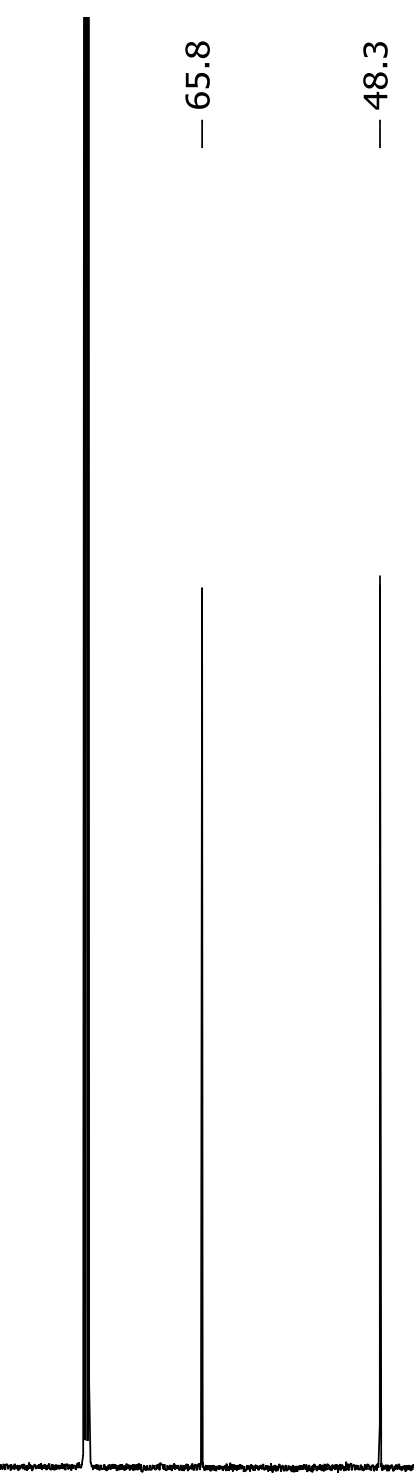




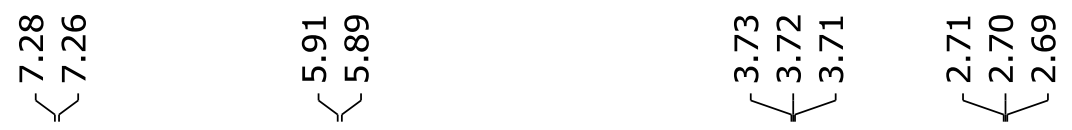

\section{$\mathrm{CDCl}_{3}, 600 \mathrm{MHz}$}
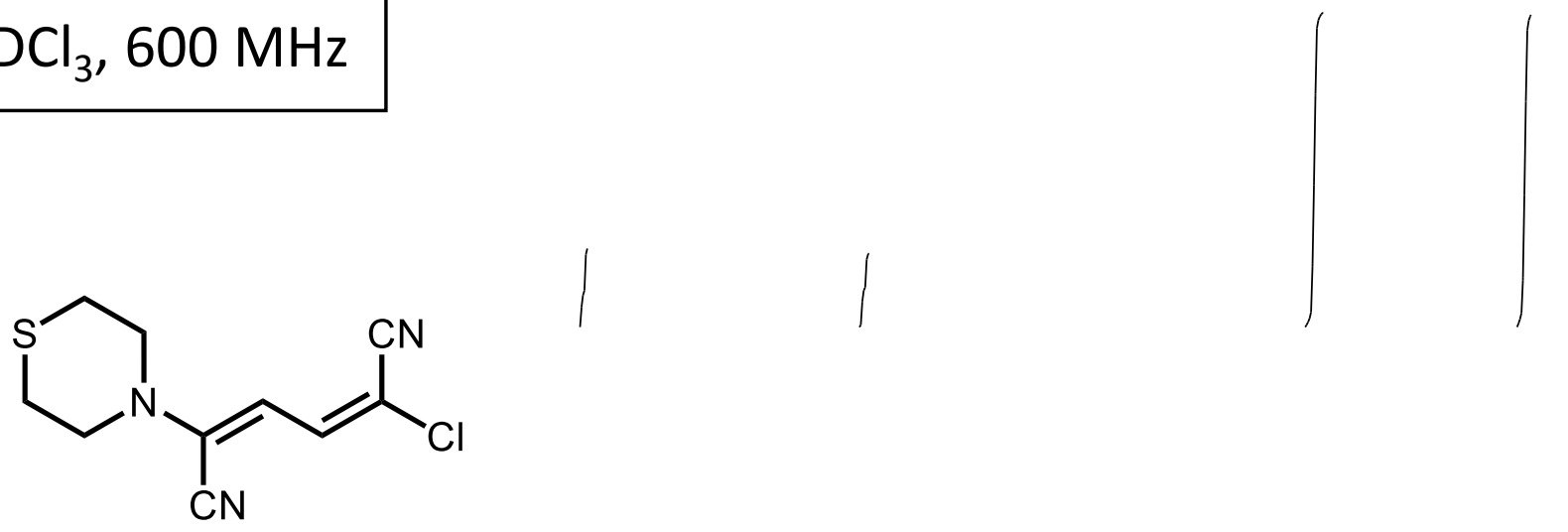

$11 a$

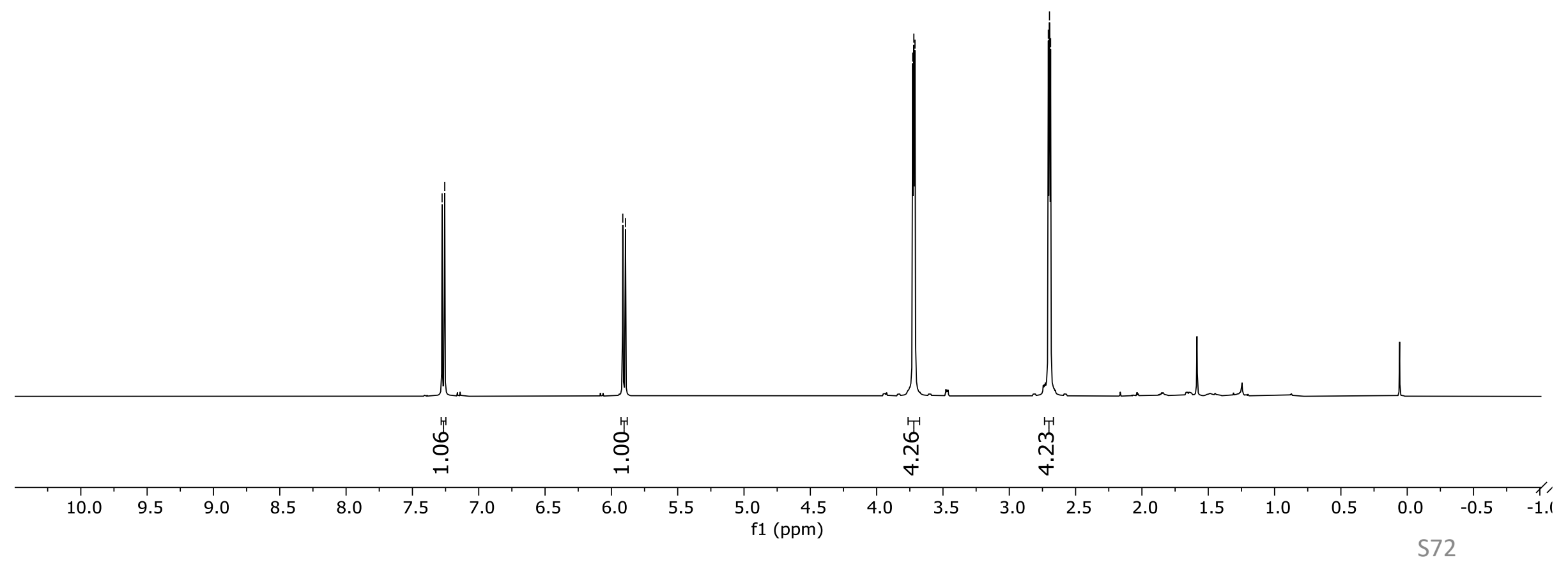




\section{$\mathrm{CDCl}_{3}, 151 \mathrm{MHz}$}

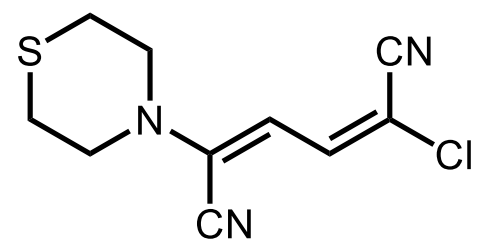

$11 a$
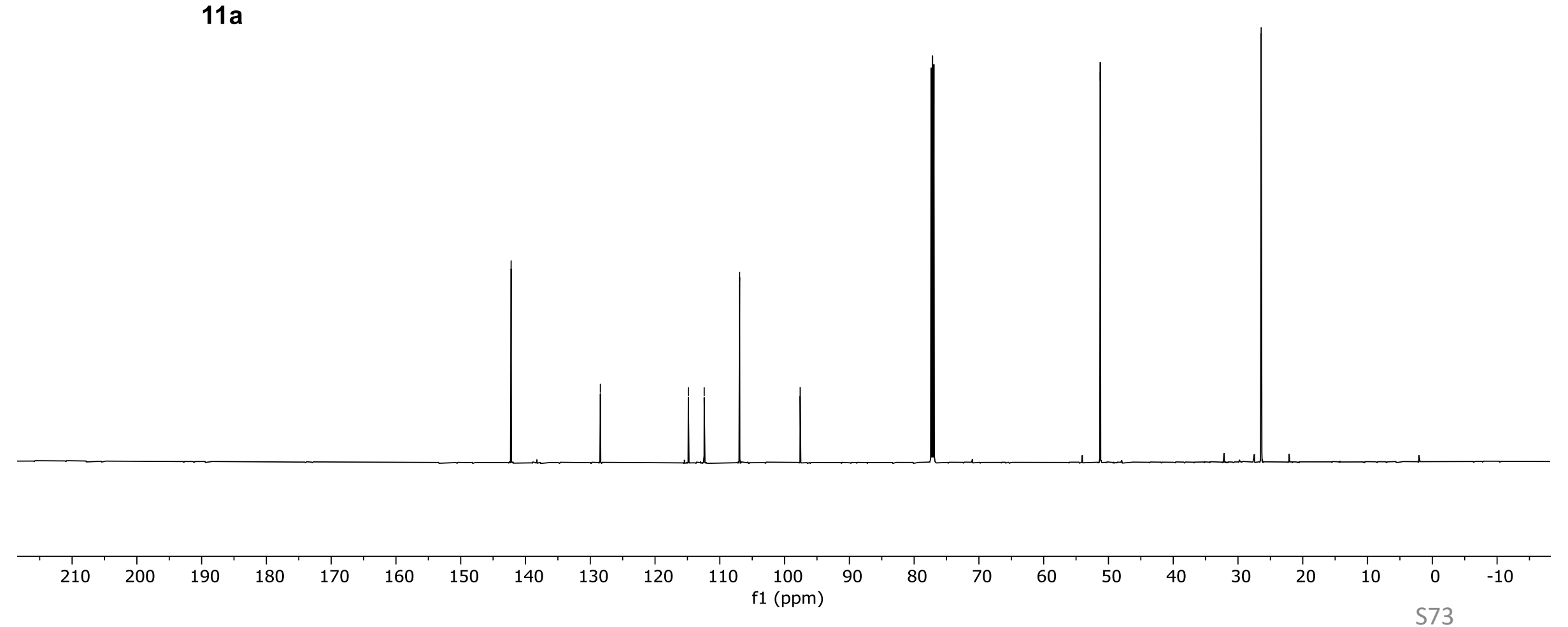


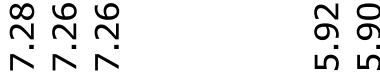

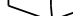

$\checkmark$

$\hat{m} \hat{m} \stackrel{m}{m}$

$m \dot{m}$

r

กับ

11

\section{$\mathrm{CDCl}_{3}, 600 \mathrm{MHz}$}
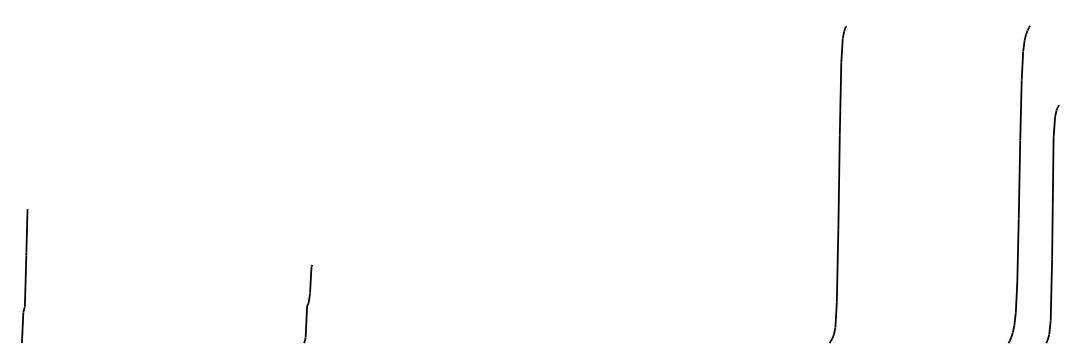

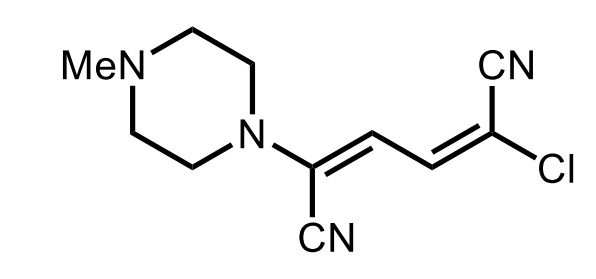

$12 a$
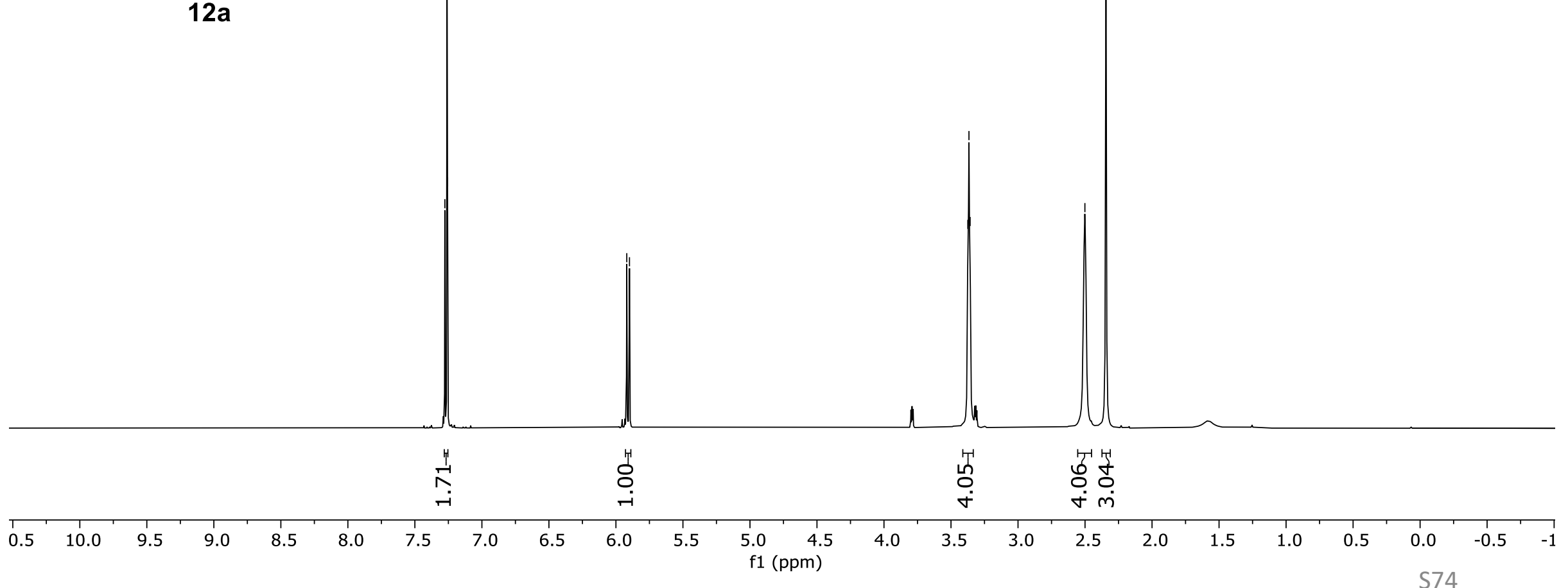


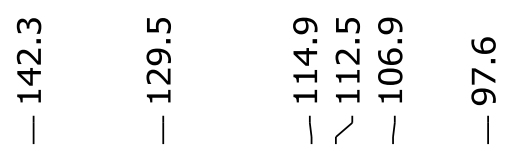

ต ำ

กิ

\section{$\mathrm{CDCl}_{3}, 151 \mathrm{MHz}$}

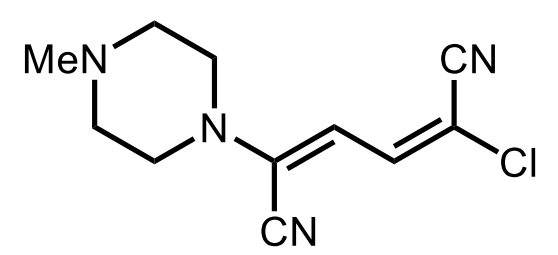

$12 a$
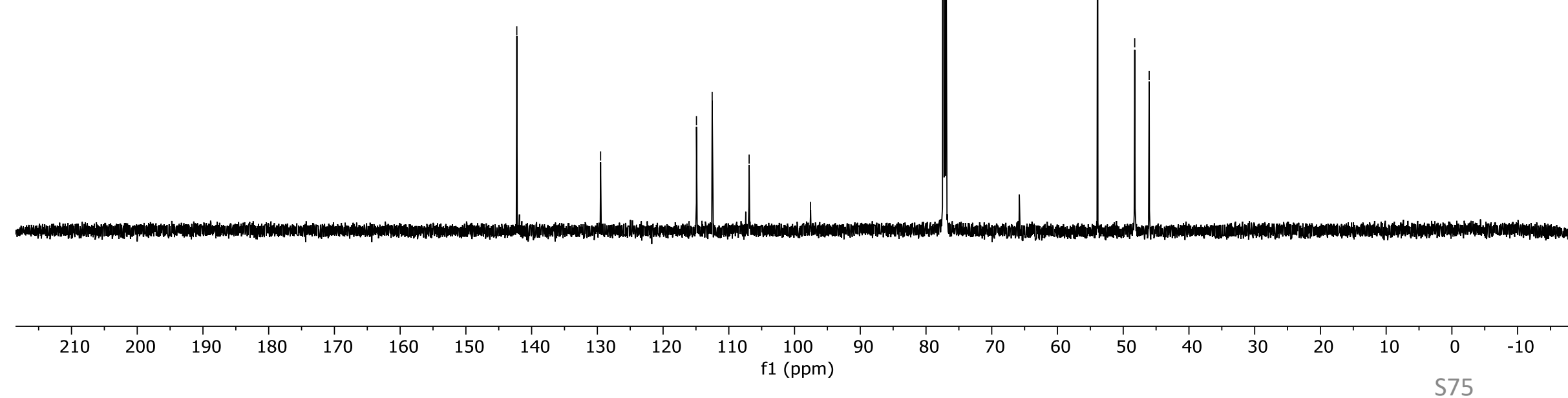


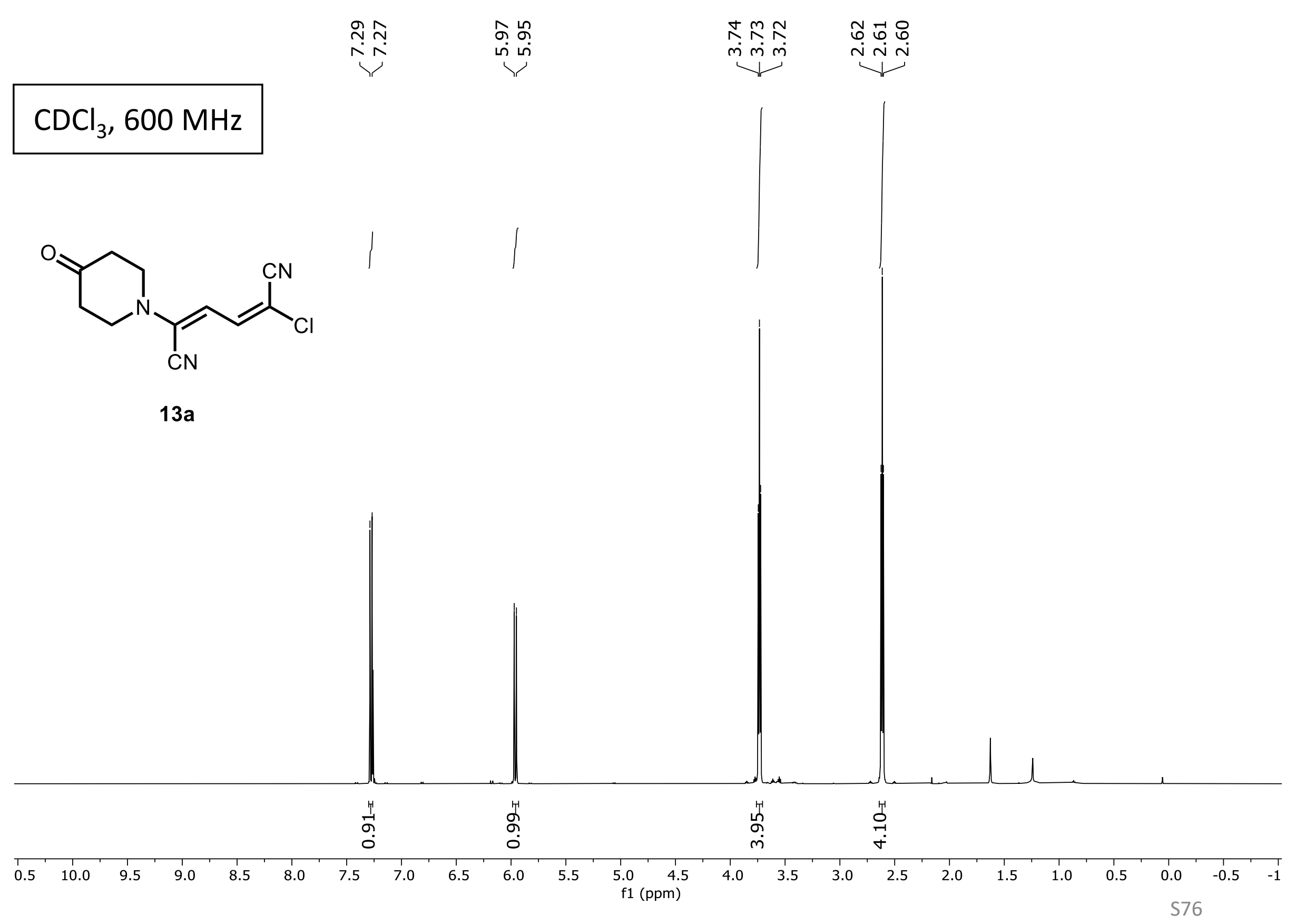




\section{$\mathrm{CDCl}_{3}, 151 \mathrm{MHz}$}

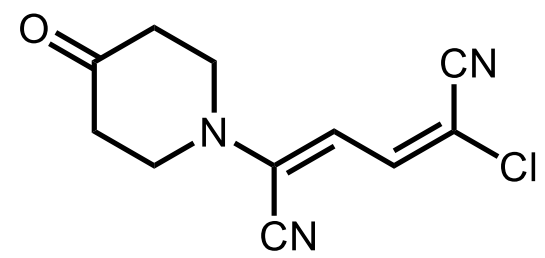

$13 a$
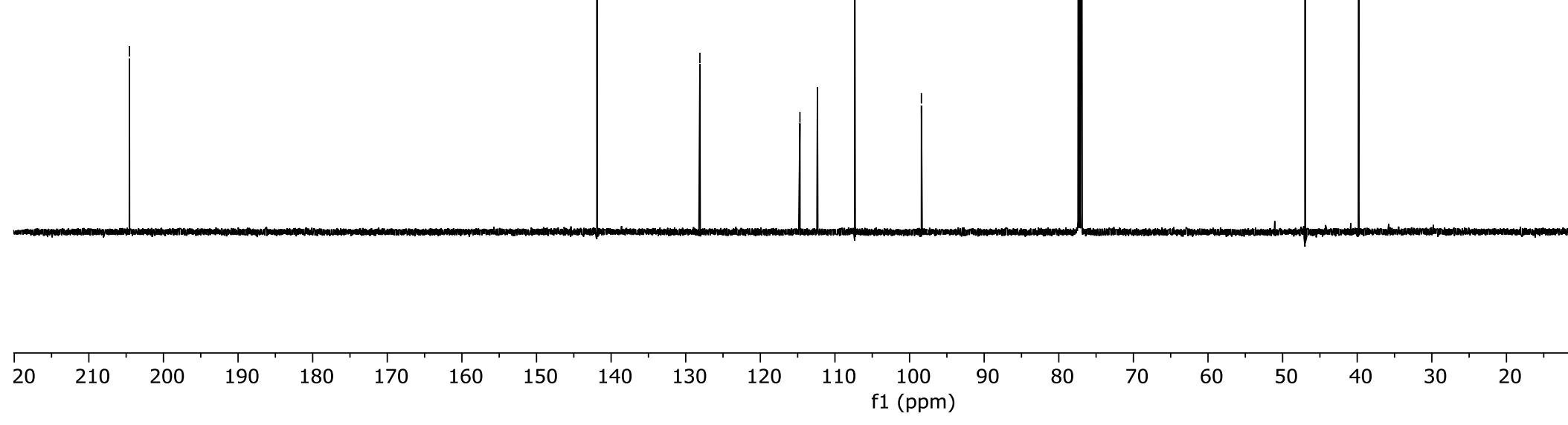


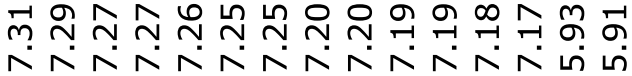

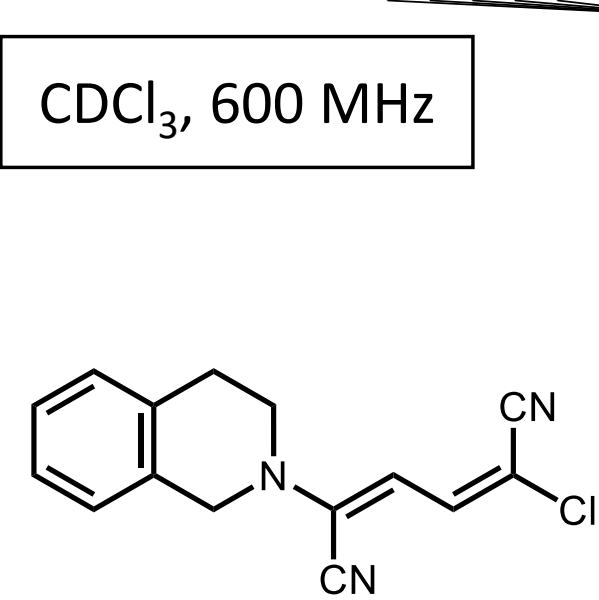

$14 a$

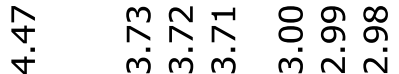

mN
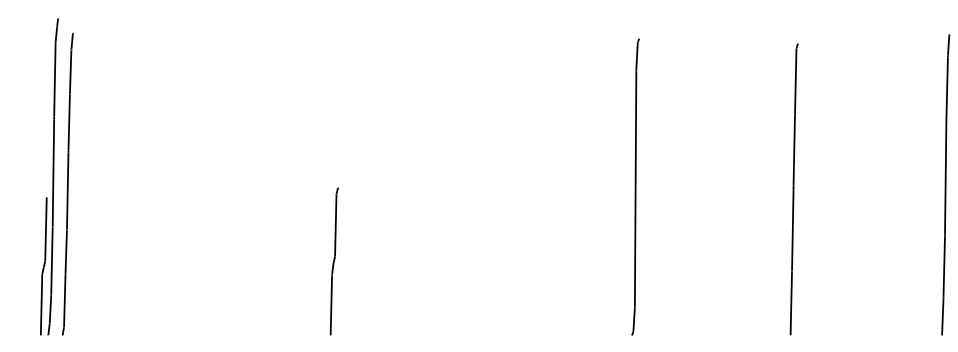

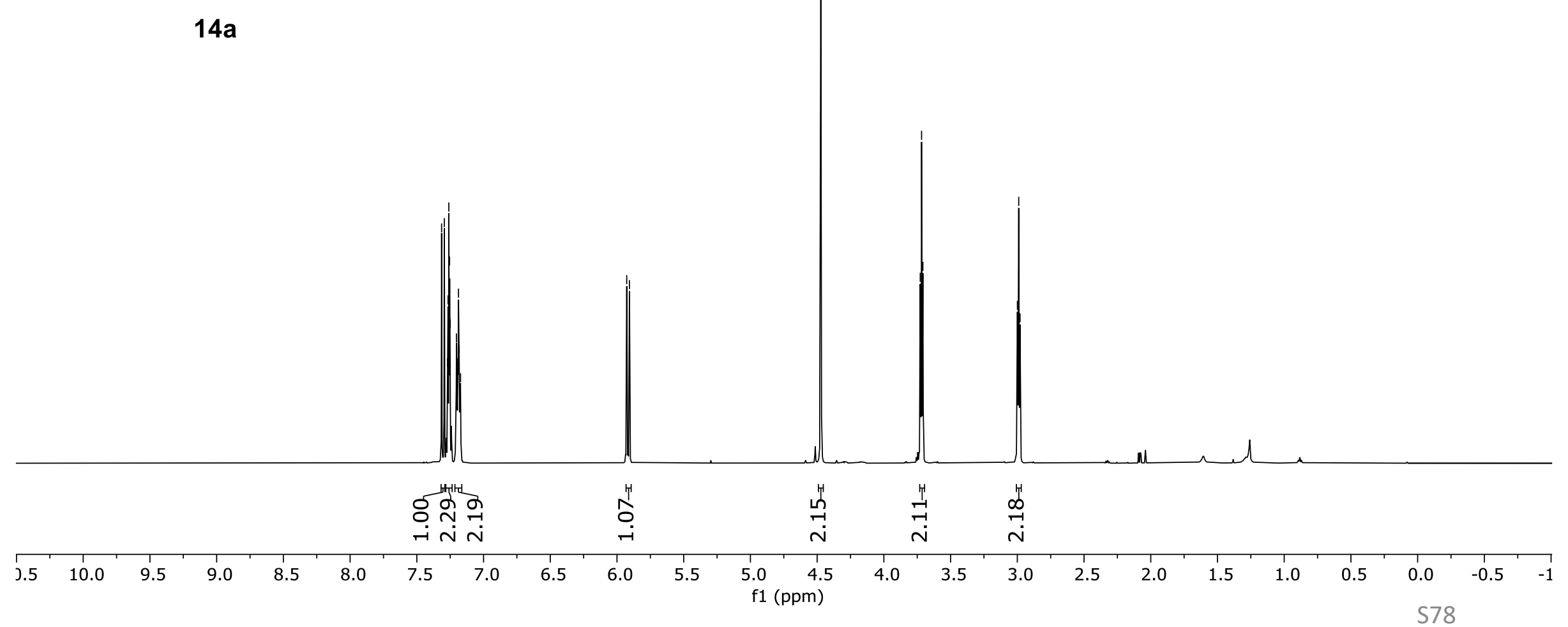




\section{$\mathrm{CDCl}_{3}, 151 \mathrm{MHz}$}

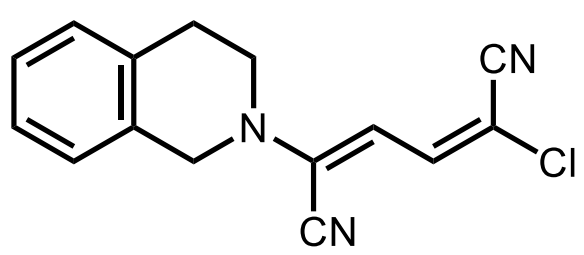

$14 a$

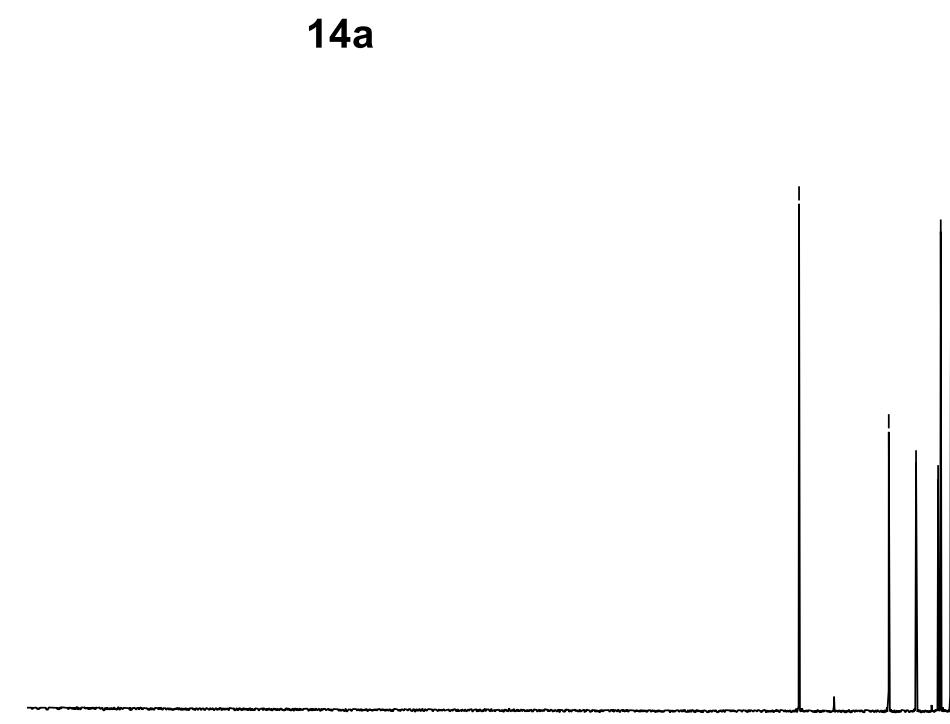

\begin{tabular}{|c|c|c|c|c|c|c|c|c|c|c|c|c|c|c|c|c|c|c|c|c|c|c|}
\hline 210 & 200 & 190 & 180 & 170 & 160 & 150 & 140 & 130 & 120 & 110 & $\begin{array}{c}100 \\
\text { f1 }(\mathrm{ppm})\end{array}$ & 90 & 80 & 70 & 60 & 50 & 40 & 30 & 20 & 10 & $\begin{array}{c}0 \\
S 79\end{array}$ & -10 \\
\hline
\end{tabular}




\section{$\mathrm{CDCl}_{3}, 600 \mathrm{MHz}$}

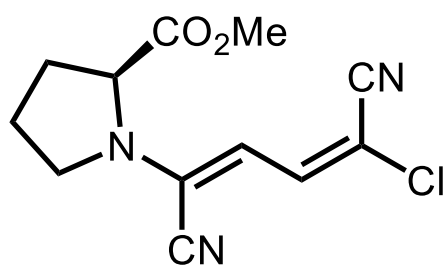

$15 a$

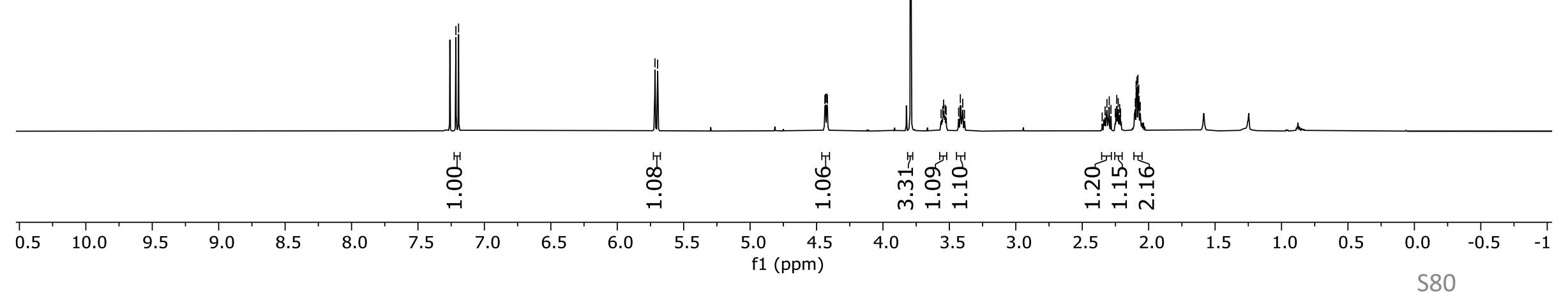



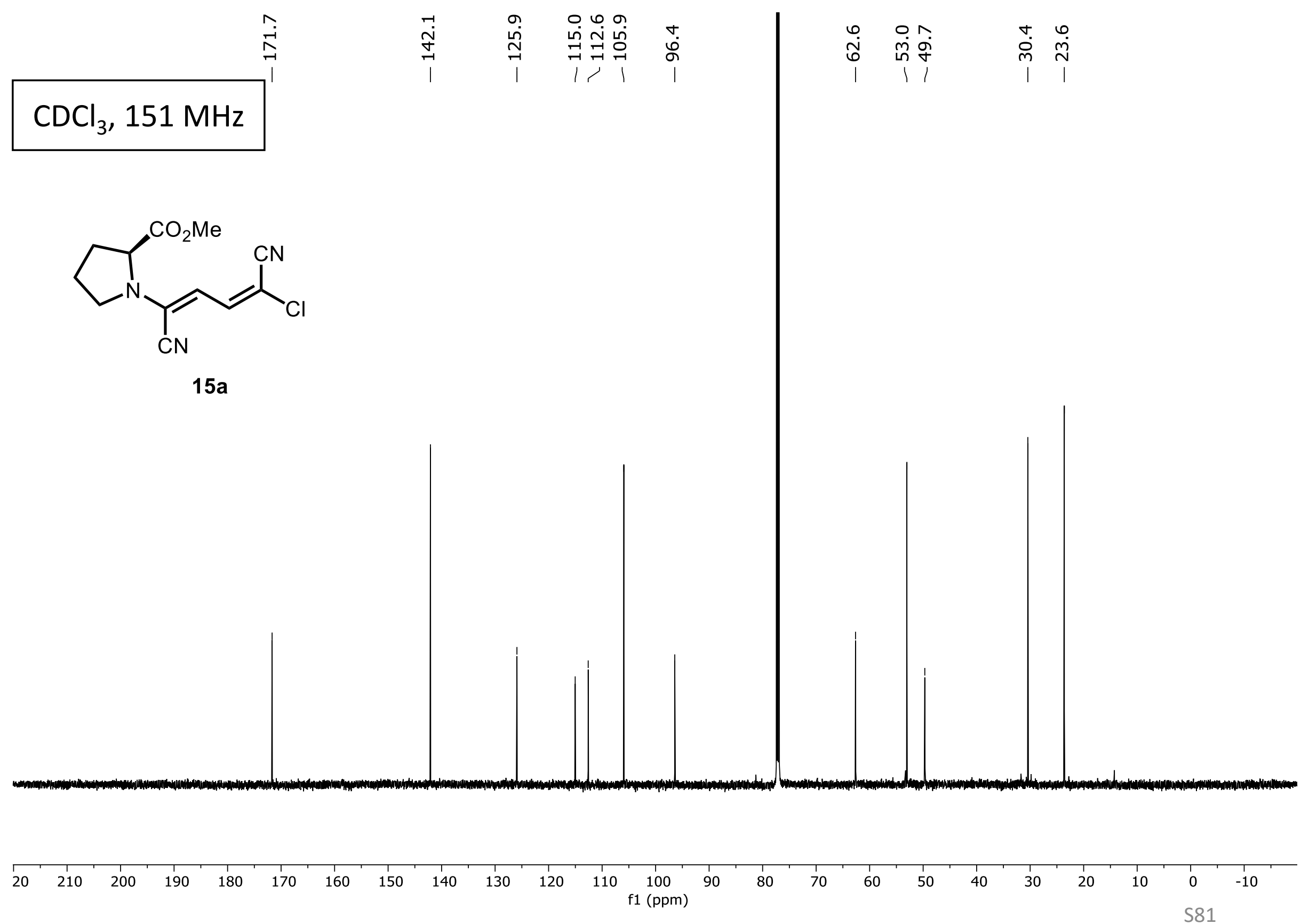


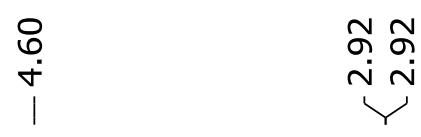

\section{$\mathrm{CDCl}_{3}, 600 \mathrm{MHz}$}
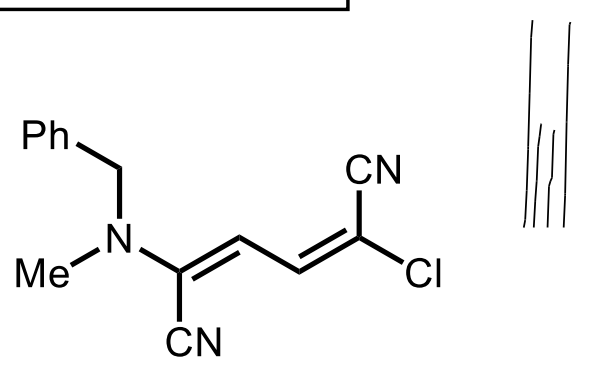

$16 a$

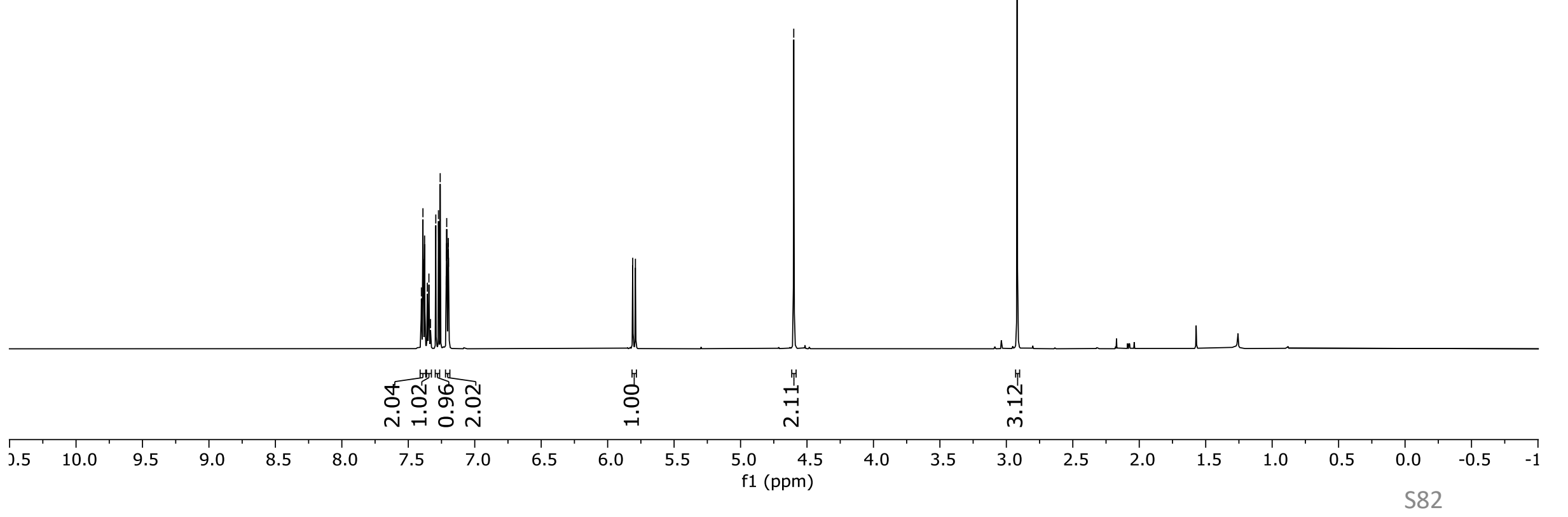




\section{$\mathrm{CDCl}_{3}, 151 \mathrm{MHz}$}<smiles>CN(Cc1ccccc1)/C(C#N)=C/C=C(\Cl)C#N</smiles>

$16 a$
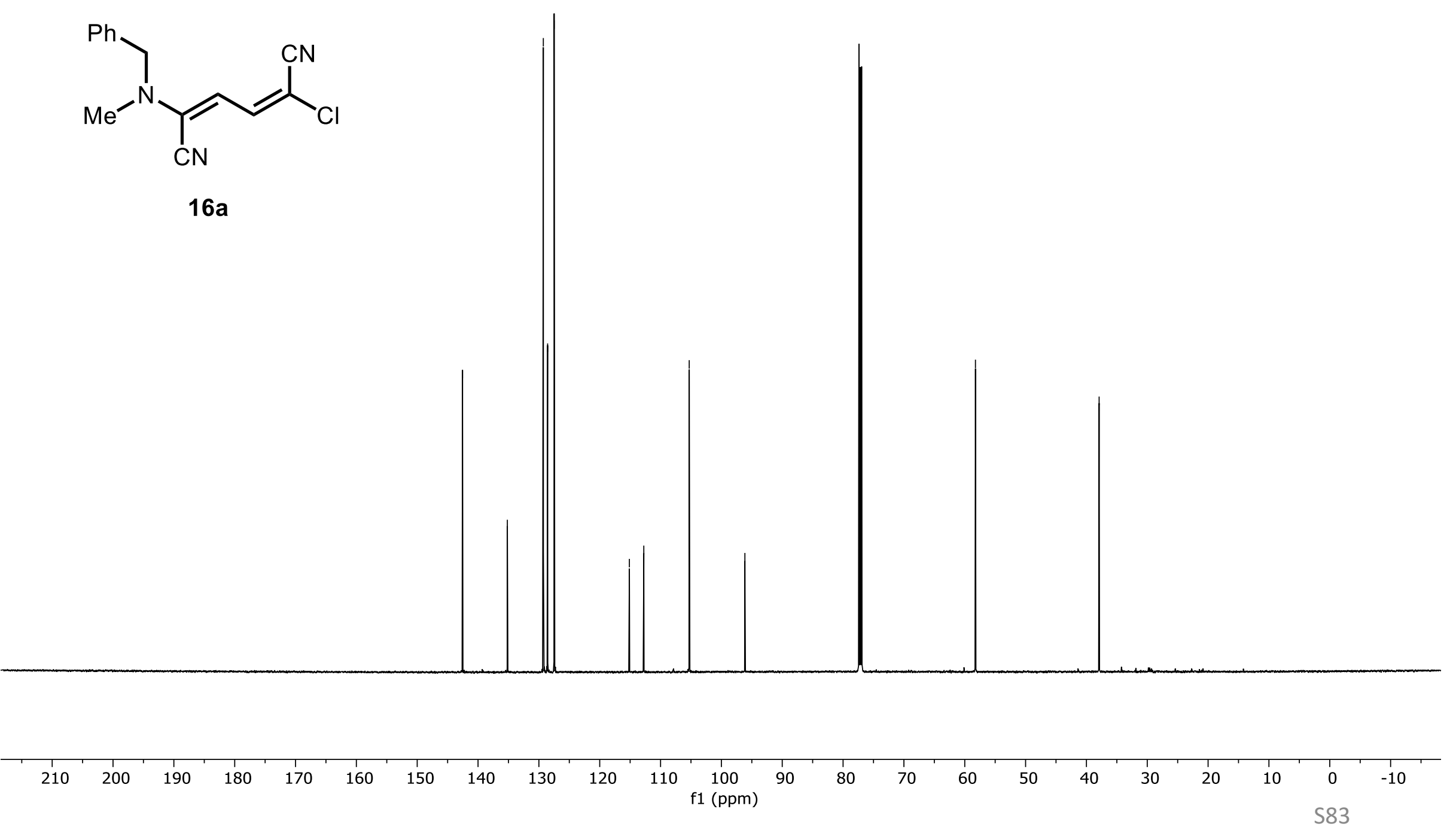
๗ัm



\section{$\mathrm{CDCl}_{3}, 600 \mathrm{MHz}$}

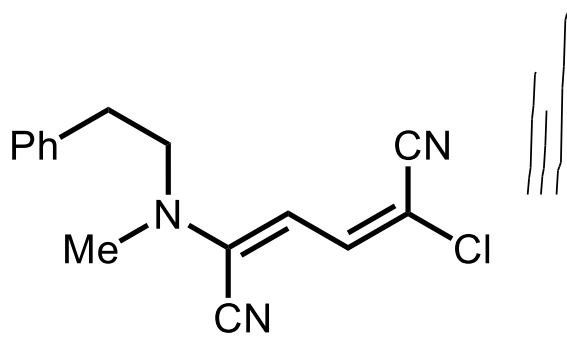

$17 a$

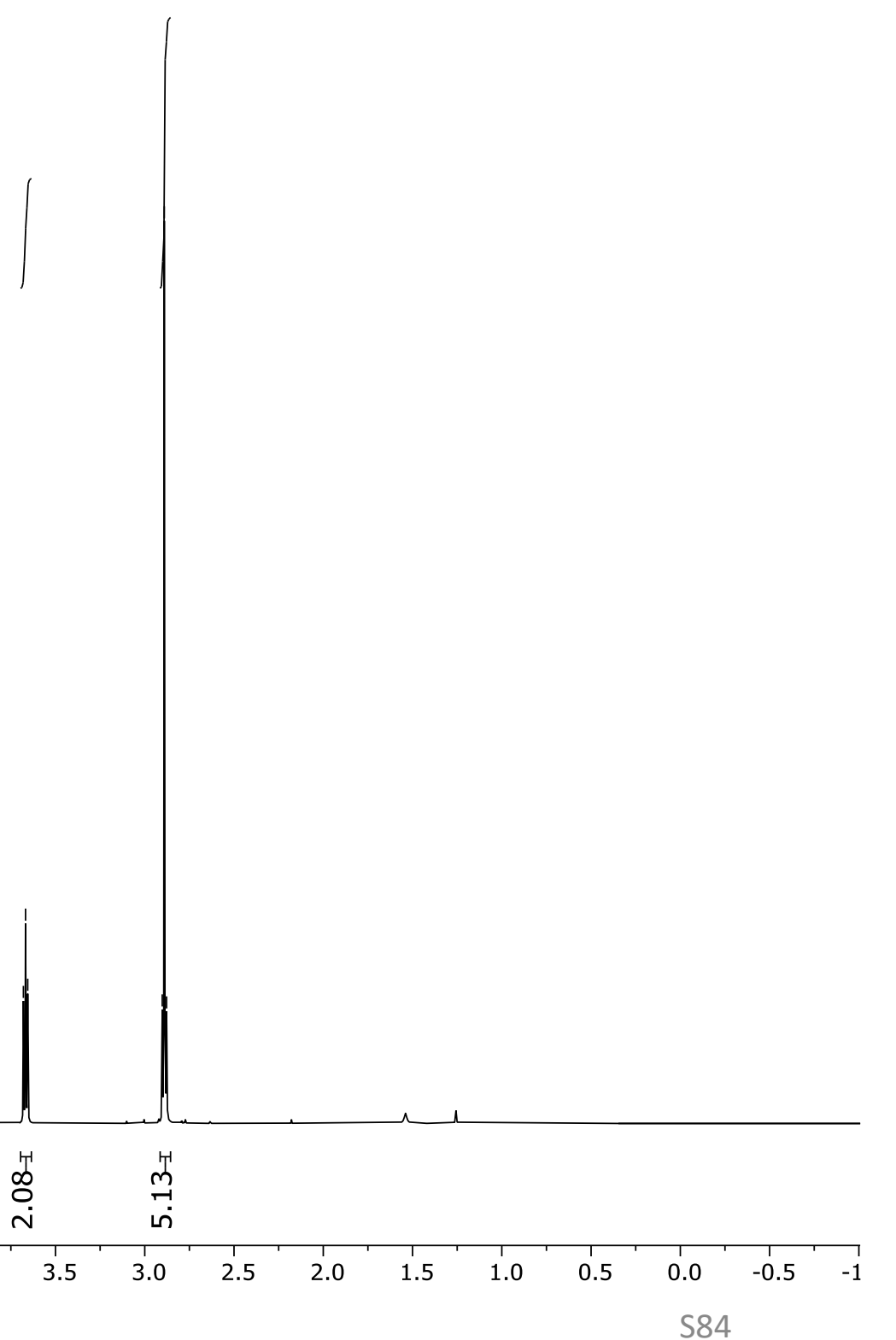




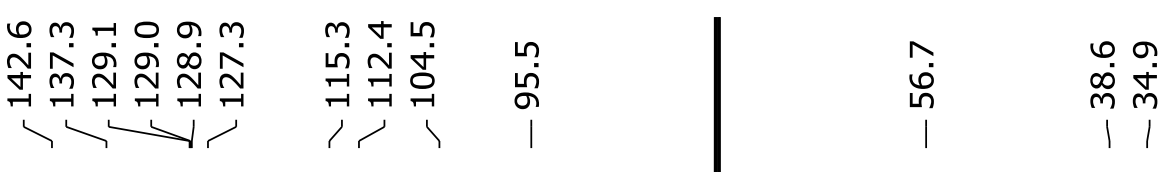

\section{$\mathrm{CDCl}_{3}, 151 \mathrm{MHz}$}<smiles>CN(CCc1ccccc1)/C(C#N)=C/C=C(\Cl)C#N</smiles>

$17 a$

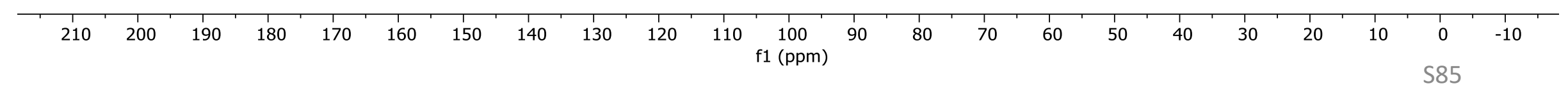




\section{$\mathrm{CDCl}_{3}, 600 \mathrm{MHz}$}<smiles>CN(C)/C(C#N)=C/C=C(\Cl)C#N</smiles>

$18 a$

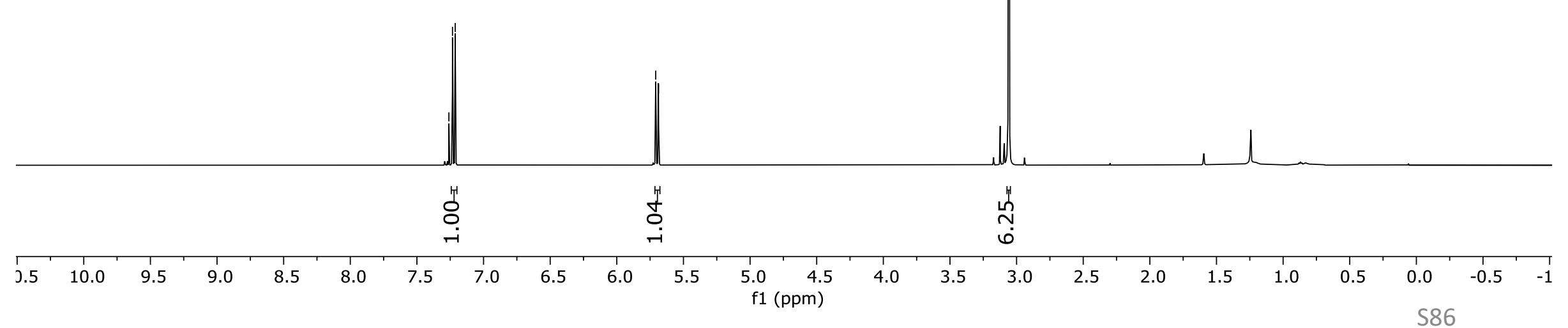




\section{$\mathrm{CDCl}_{3}, 151 \mathrm{MHz}$}<smiles>CN(C)/C(C#N)=C/C=C(\Cl)C#N</smiles>

$18 a$

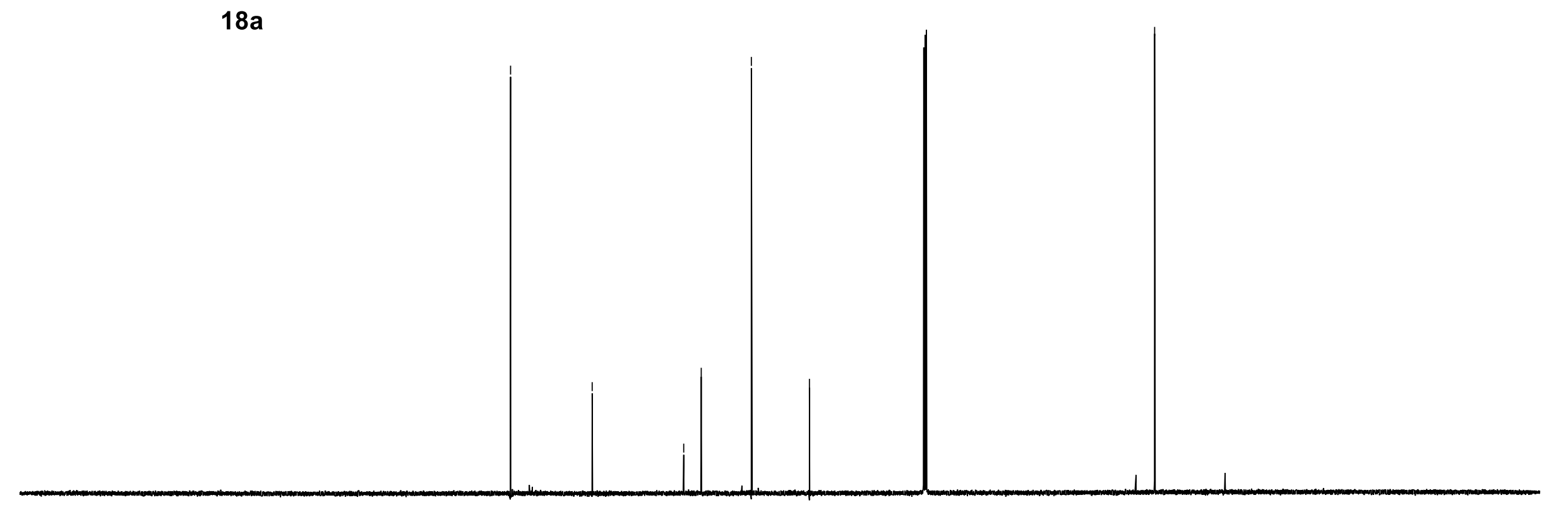




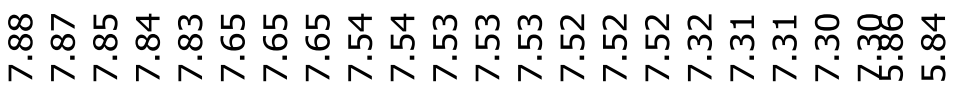

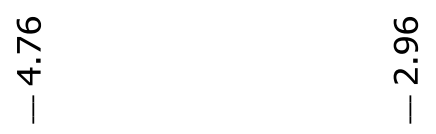

\section{$\mathrm{CDCl}_{3}, 600 \mathrm{MHz}$}
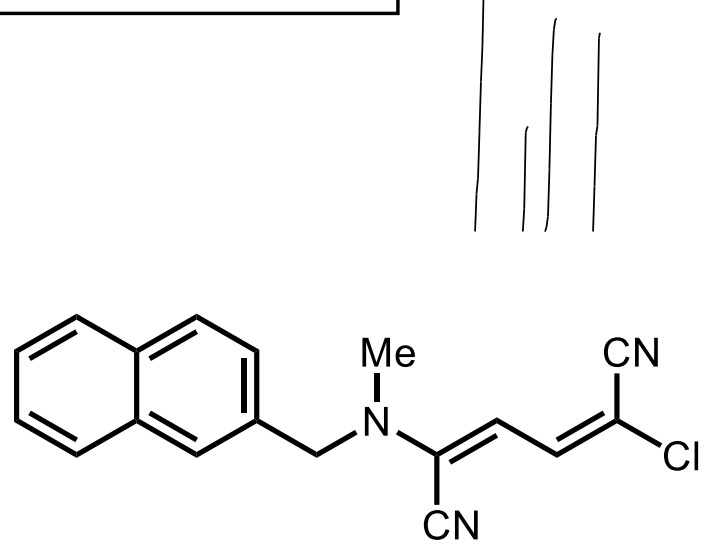

$19 a$

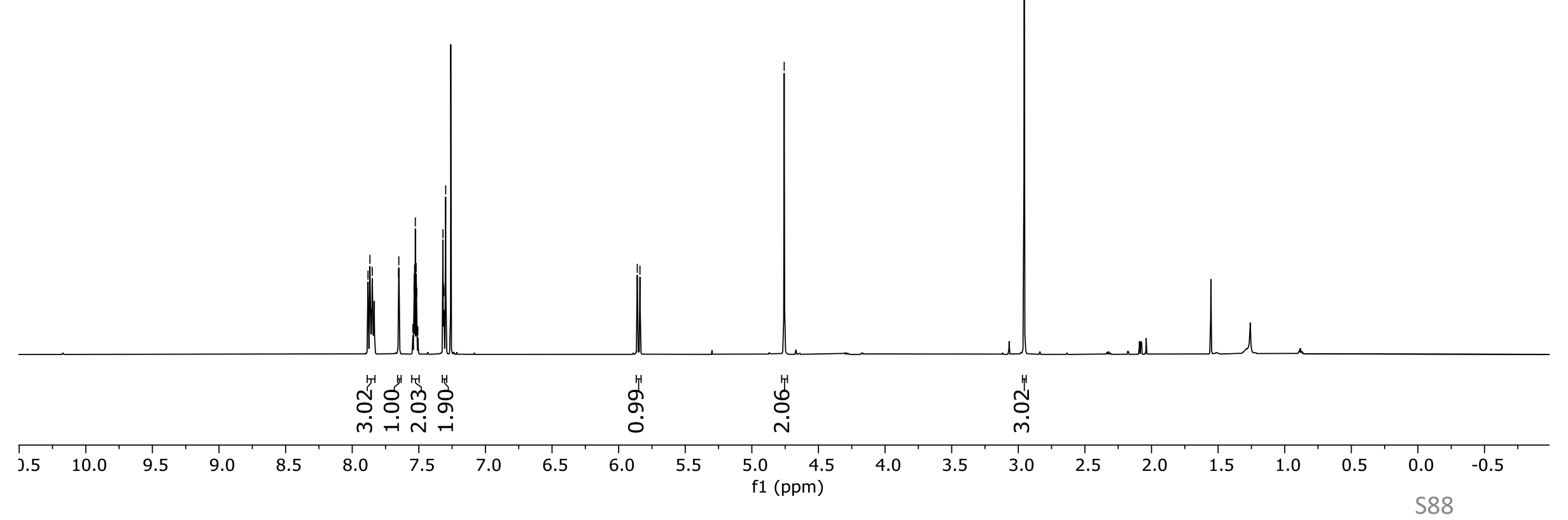


๑

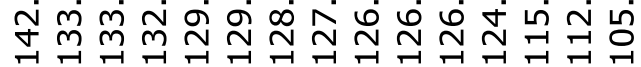

\section{:}

!n

m

\section{$\mathrm{CDCl}_{3}, 151 \mathrm{MHz}$}<smiles>CN(Cc1ccc2ccccc2c1)/C(C#N)=C/C=C(\Cl)C#N</smiles>

$19 a$
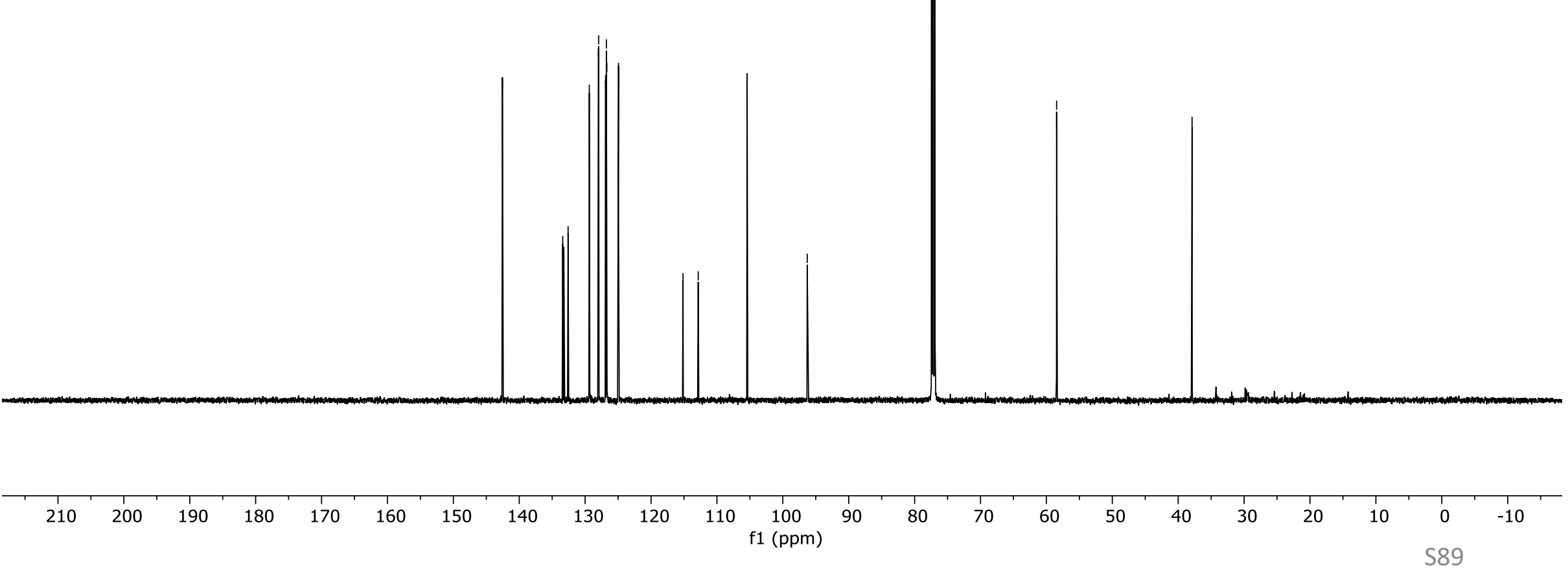


\begin{tabular}{|c|c|c|}
\hline 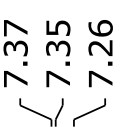 & 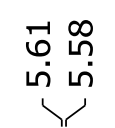 & 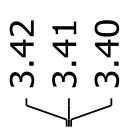 \\
\hline
\end{tabular}

\section{$\mathrm{CDCl}_{3}, 600 \mathrm{MHz}$}
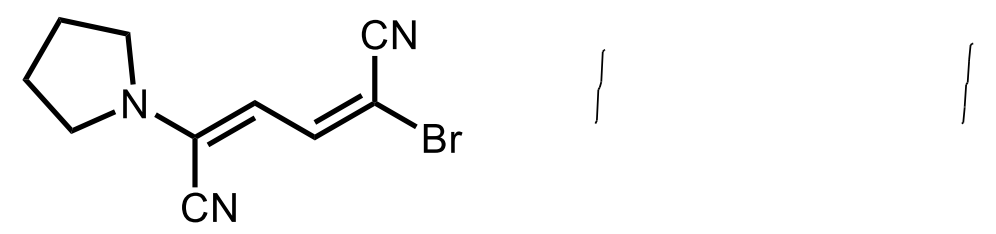

$7 b$

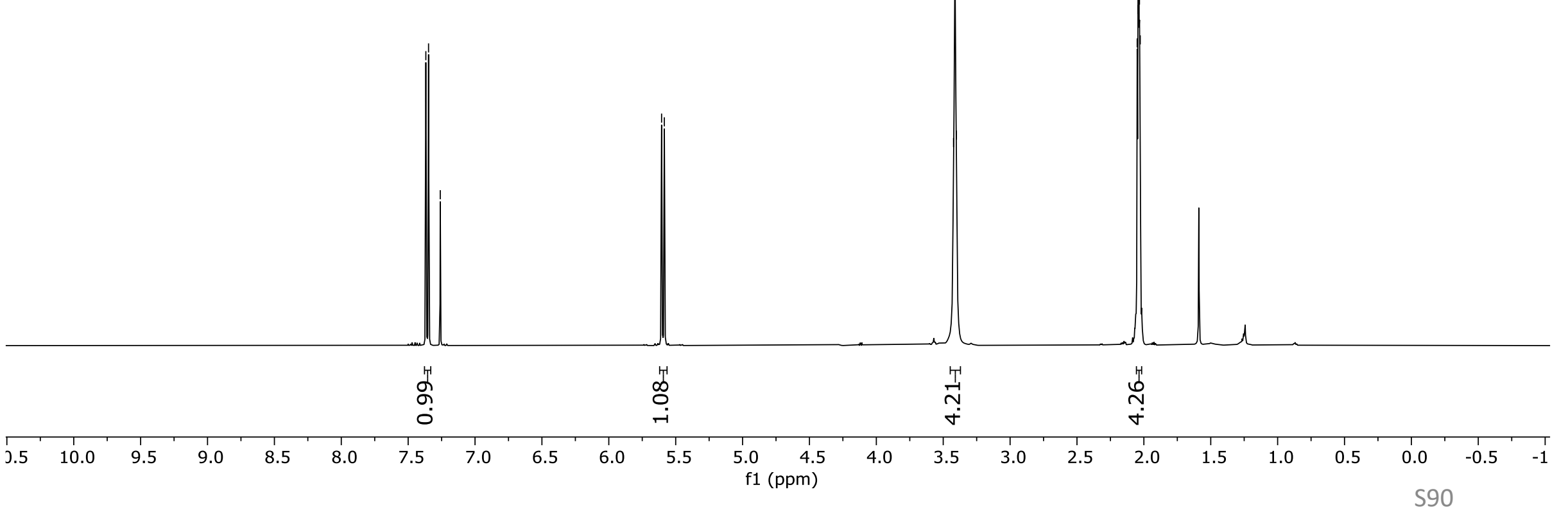




\section{$\mathrm{CDCl}_{3}, 151 \mathrm{MHz}$}<smiles>N#C/C(Br)=C\C=C(/C#N)N1CCCC1</smiles>

$7 b$

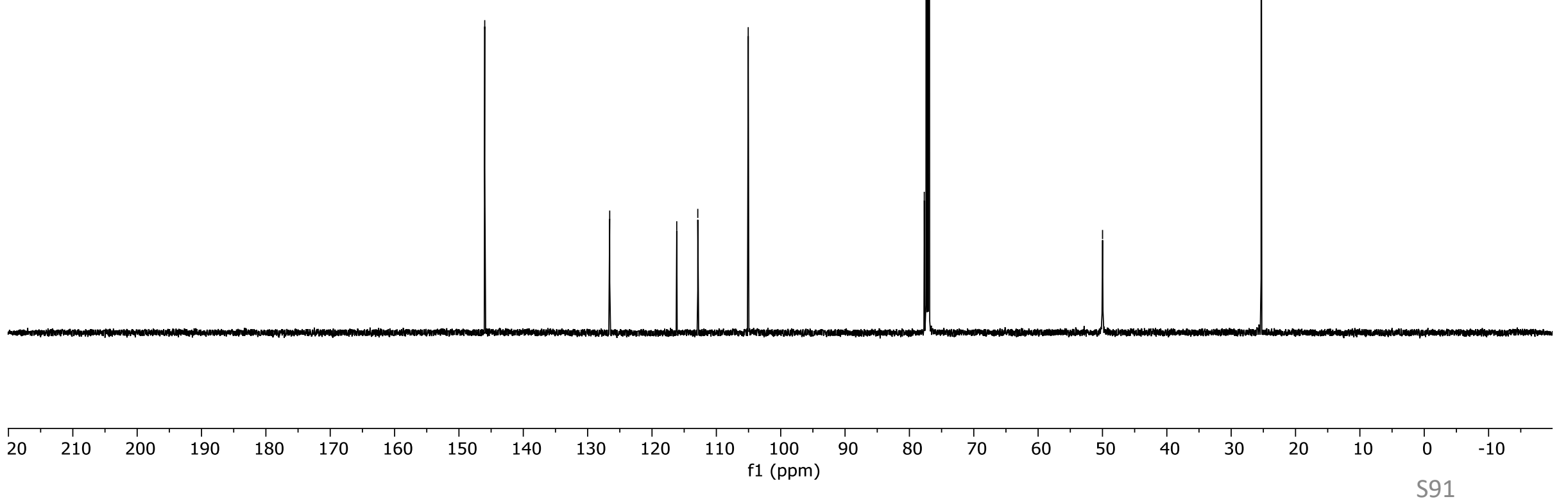




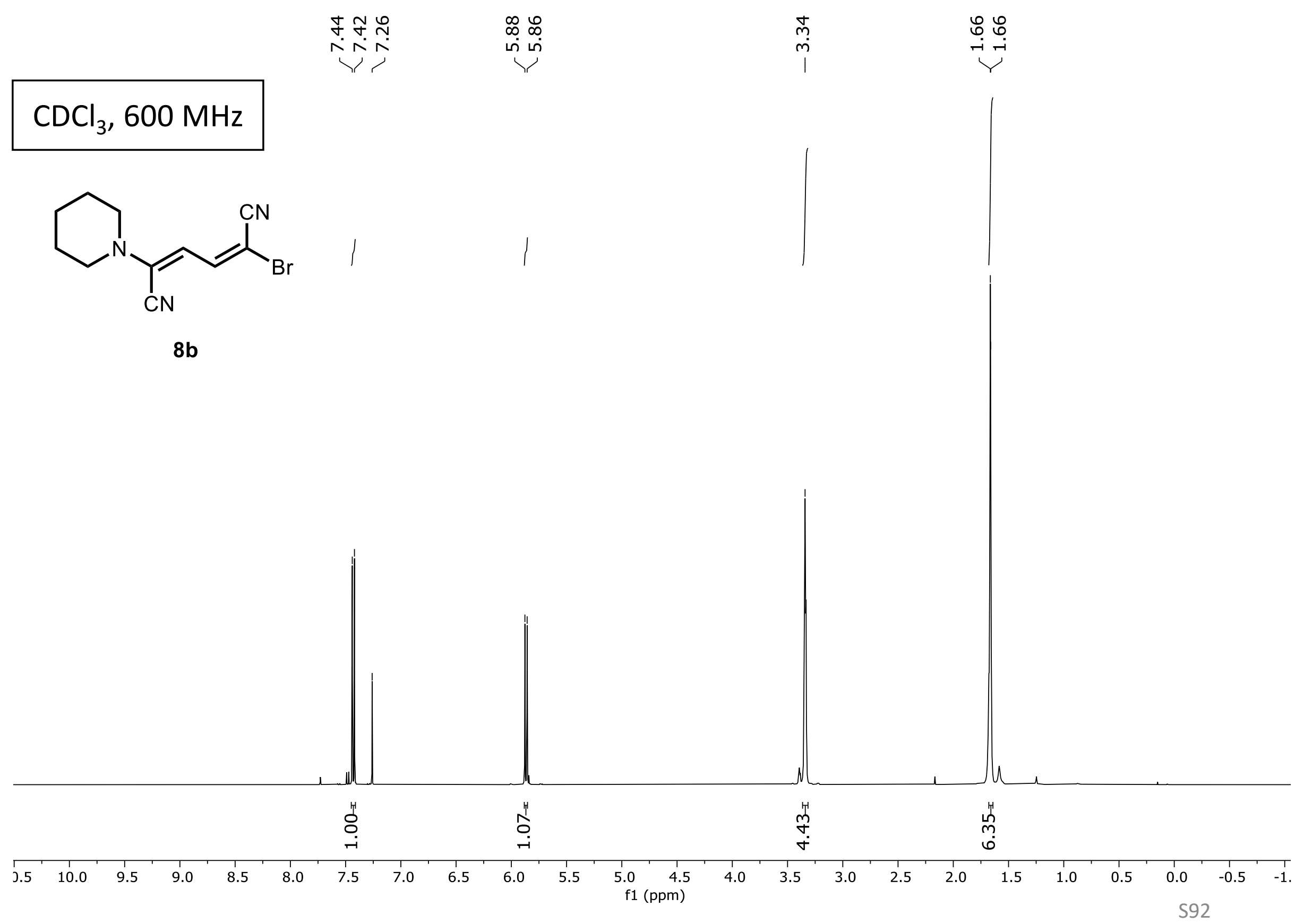




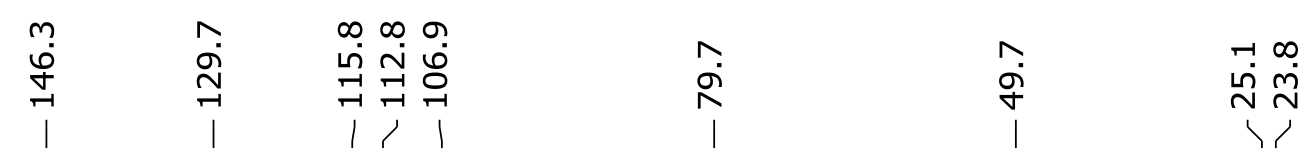

\section{$\mathrm{CDCl}_{3}, 151 \mathrm{MHz}$}

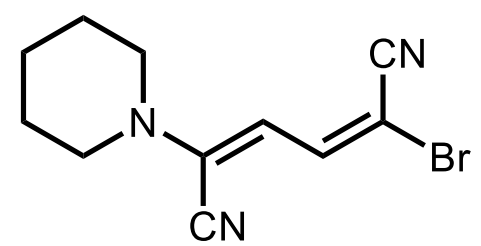

$8 b$

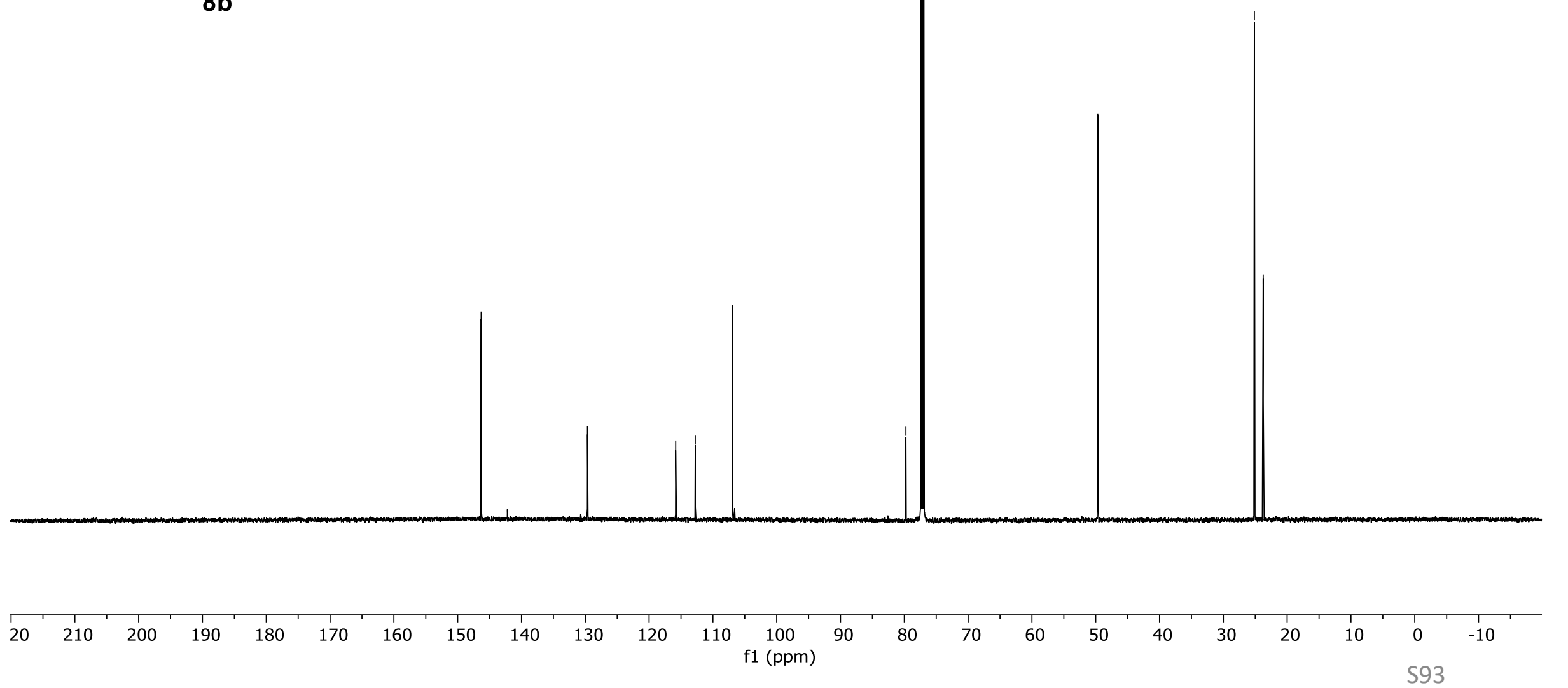




\begin{tabular}{|c|c|c|c|}
\hline & $\begin{array}{l}\text { Ni } \\
\text { in }\end{array}$ & 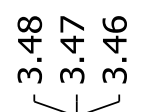 & 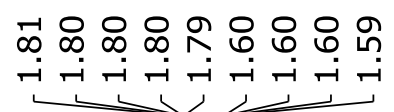 \\
\hline
\end{tabular}

\section{$\mathrm{CDCl}_{3}, 600 \mathrm{MHz}$}
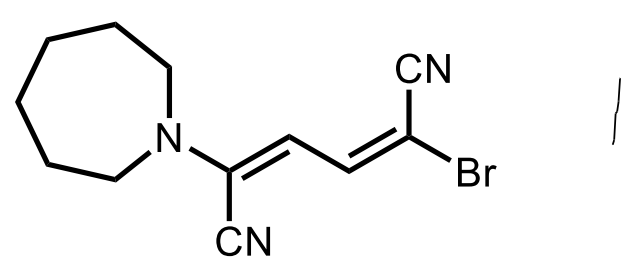

$9 b$

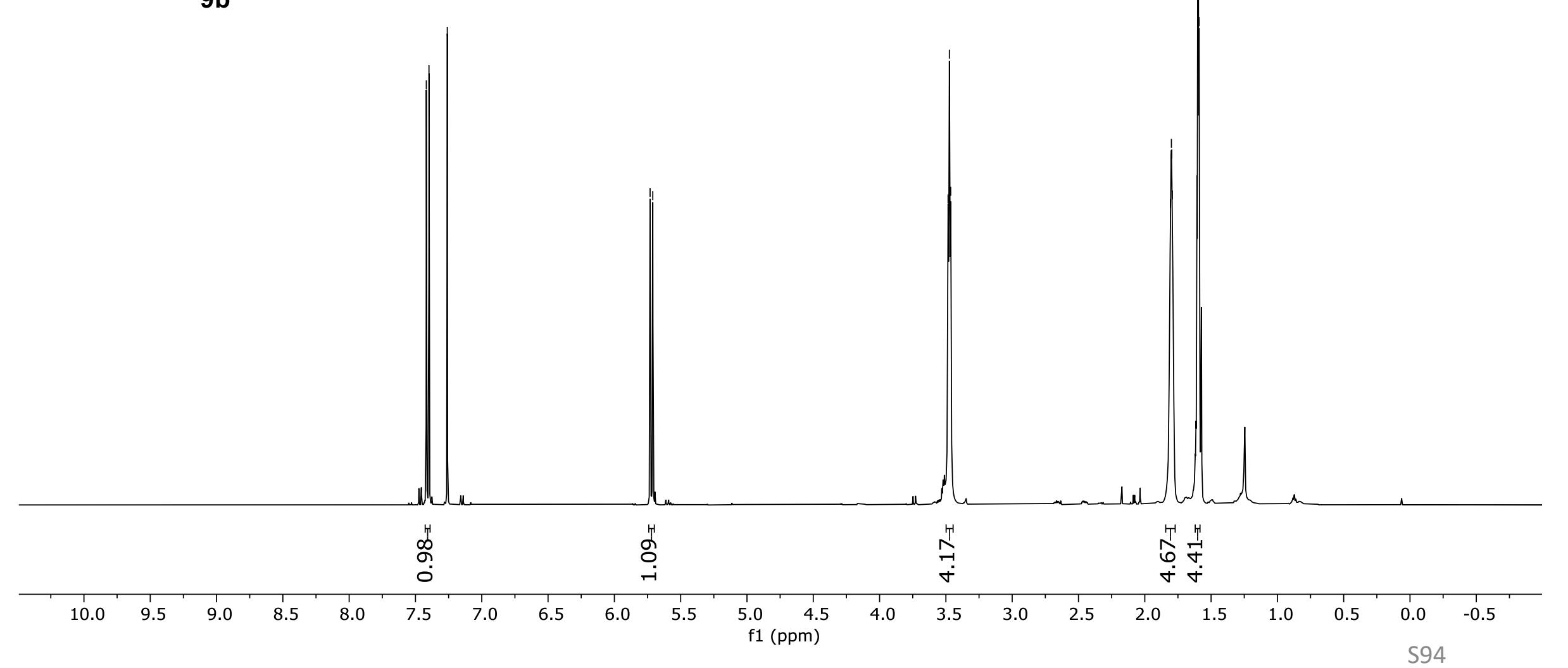




\section{$\mathrm{CDCl}_{3}, 151 \mathrm{MHz}$}<smiles>N#C/C(Br)=C\C=C(/C#N)N1CCCCCC1</smiles>

$9 b$

$$
20
$$

$210200 \quad 190$

$190 \quad 180$

$170 \quad 160$

$150 \quad 140$

$130 \quad 120$

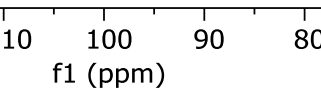

70

60

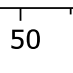

40 


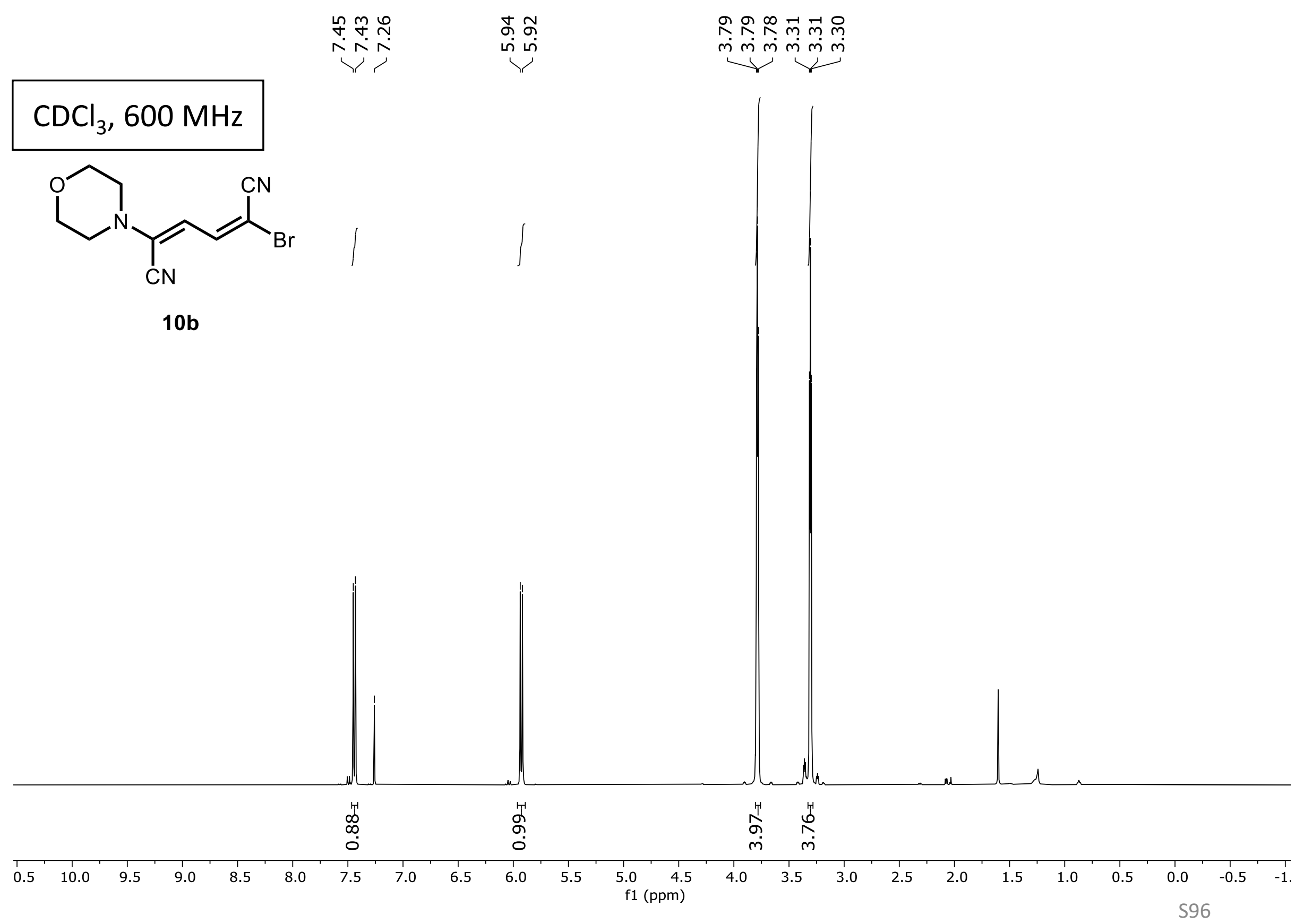



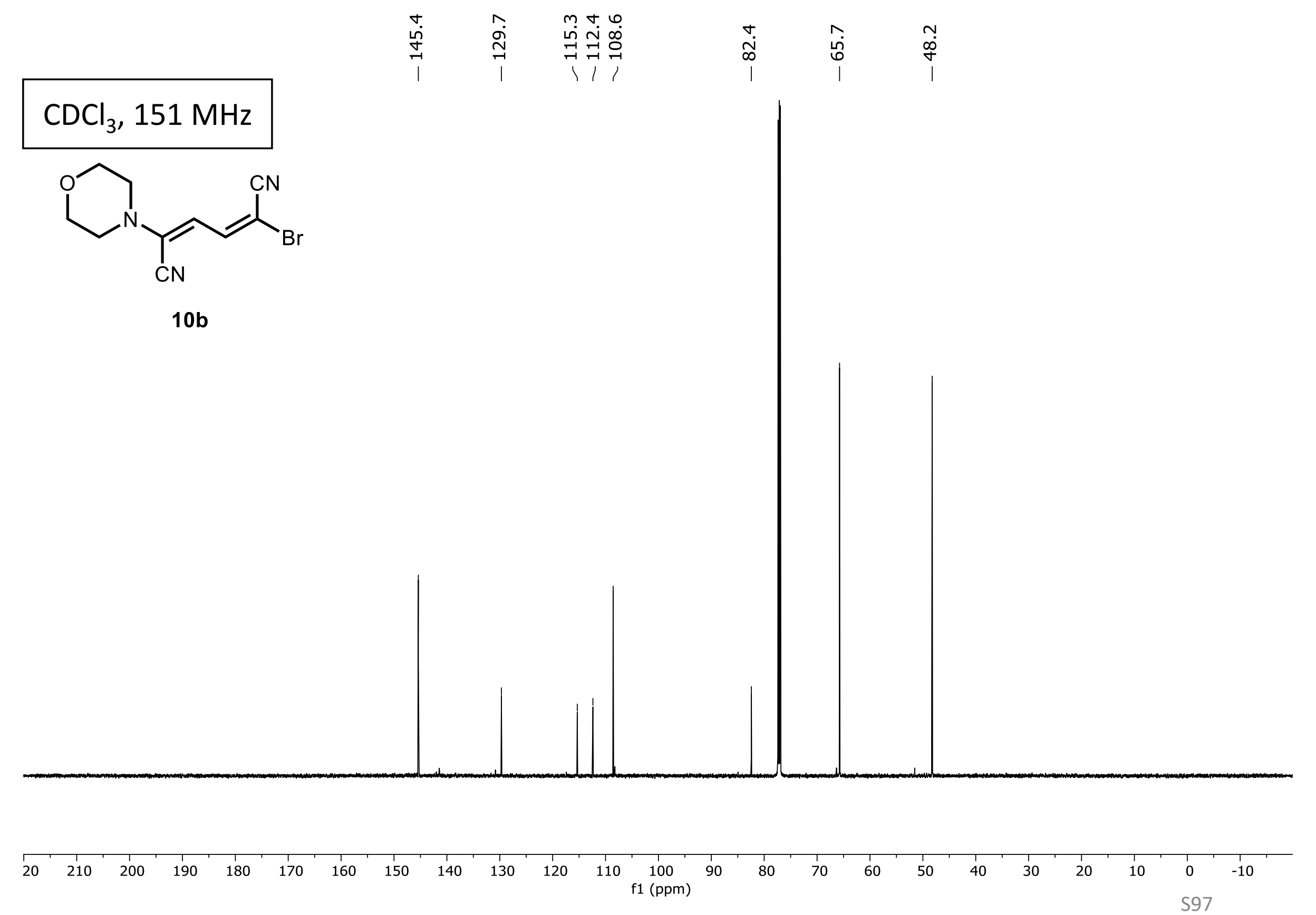


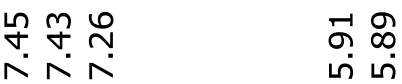

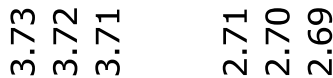

\?

$\checkmark$

$\sqrt{2}$

\section{$\mathrm{CDCl}_{3}, 600 \mathrm{MHz}$}
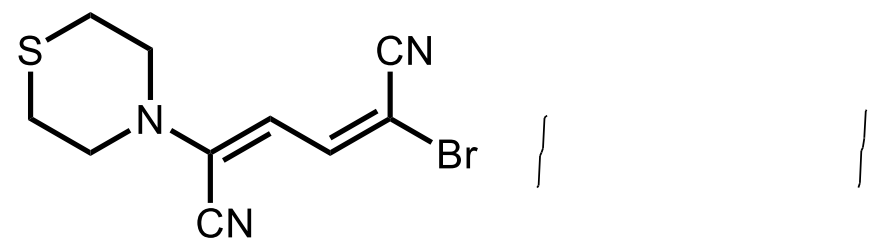

11b

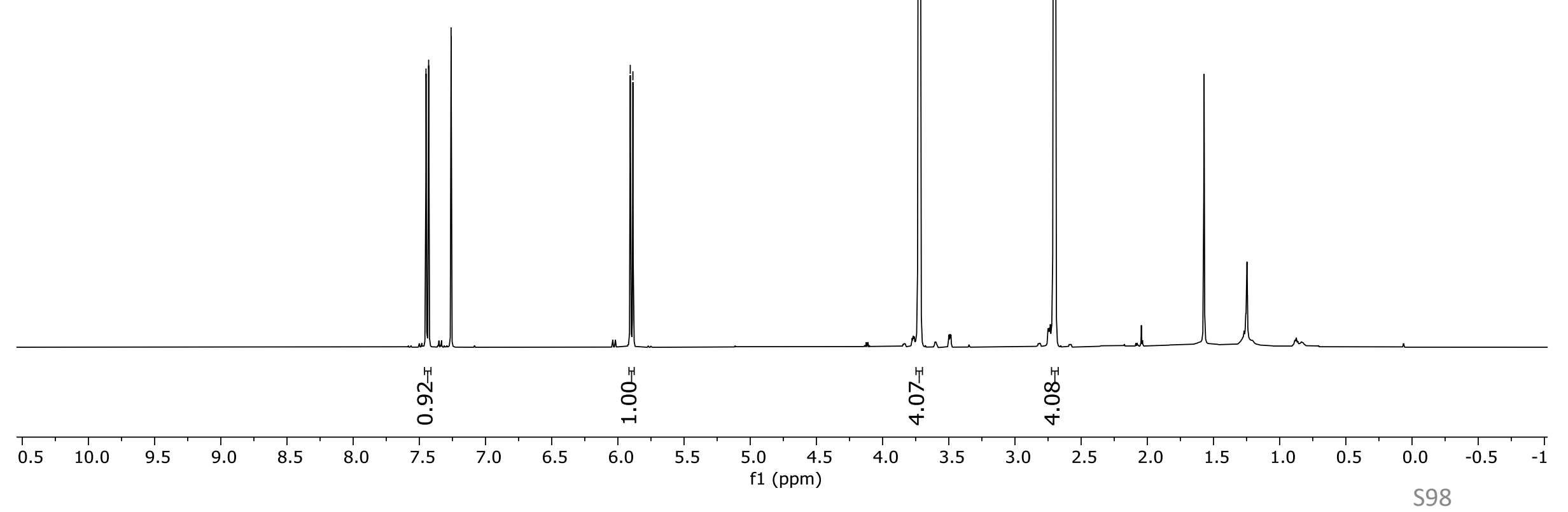




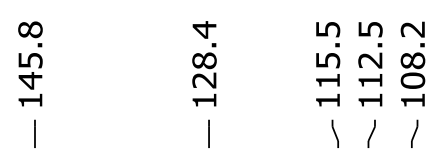

$\underset{\infty}{\infty}$

\section{$\mathrm{CDCl}_{3}, 151 \mathrm{MHz}$}

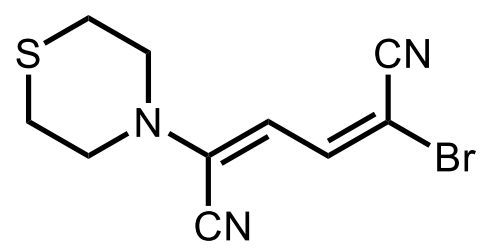

$11 b$

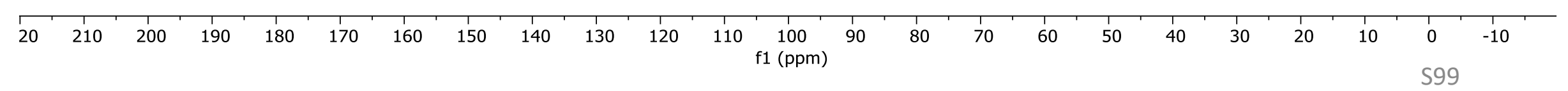




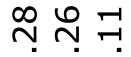

$N N N$

\)

\section{$\mathrm{CDCl}_{3}, 600 \mathrm{MHz}$}

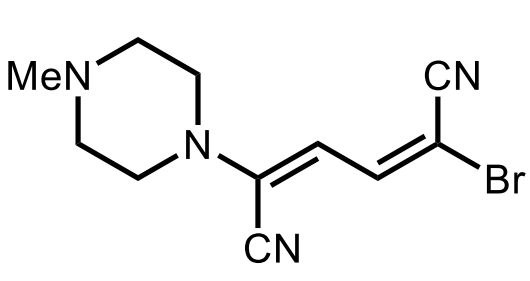

$12 b$
ำ

$\checkmark$

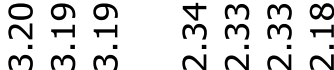

$\checkmark$

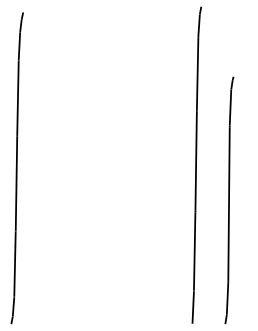

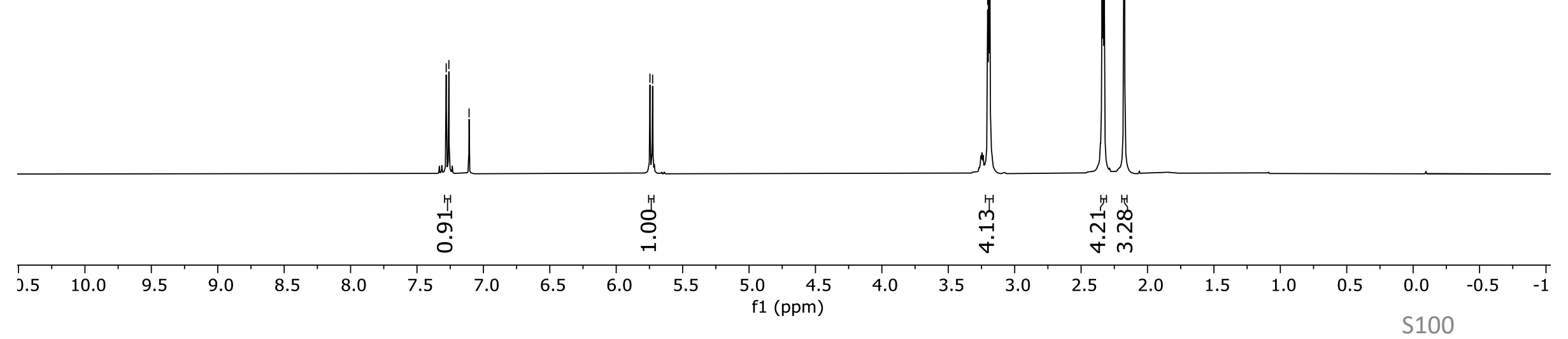




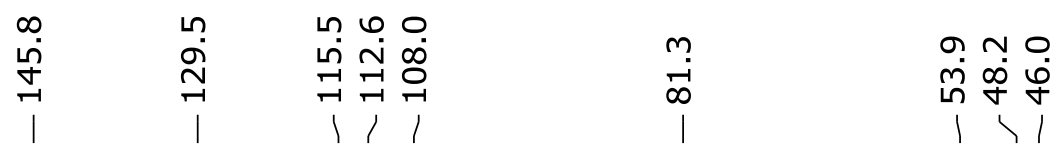

\section{$\mathrm{CDCl}_{3}, 151 \mathrm{MHz}$}

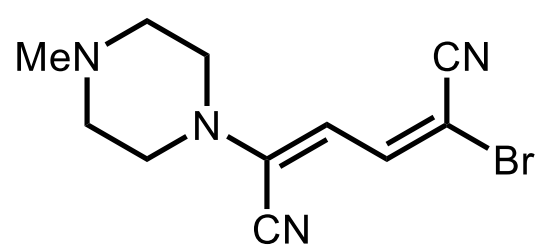

$12 b$
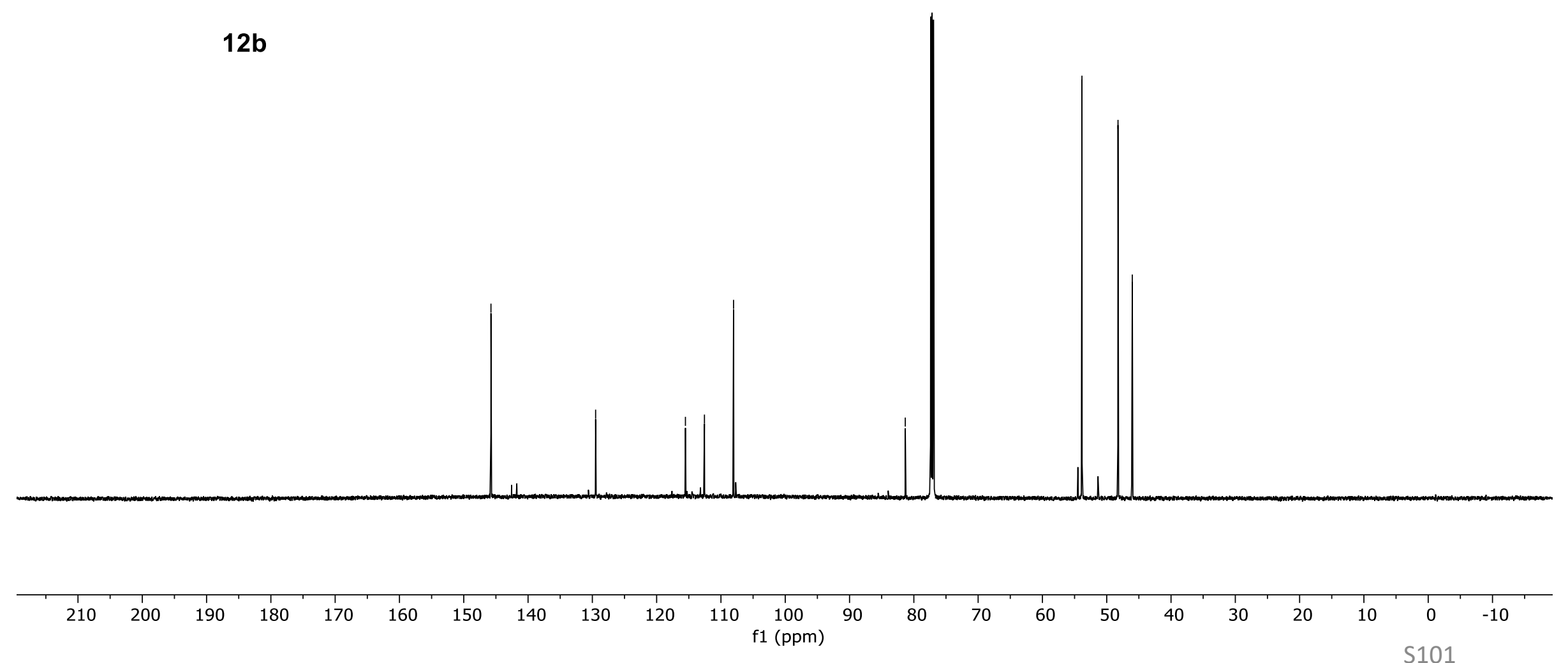


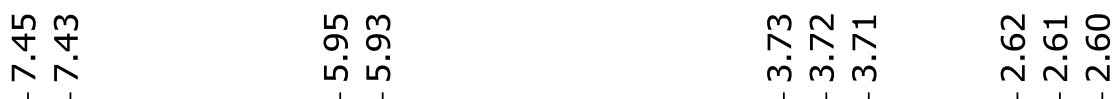

\section{$\mathrm{CDCl}_{3}, 600 \mathrm{MHz}$}

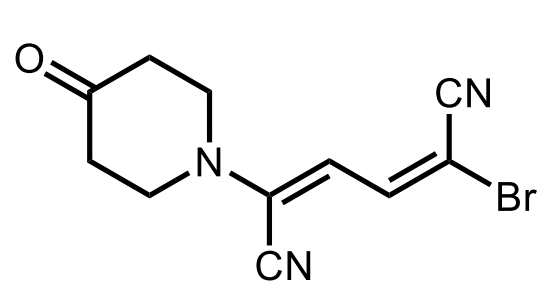

in

$13 b$

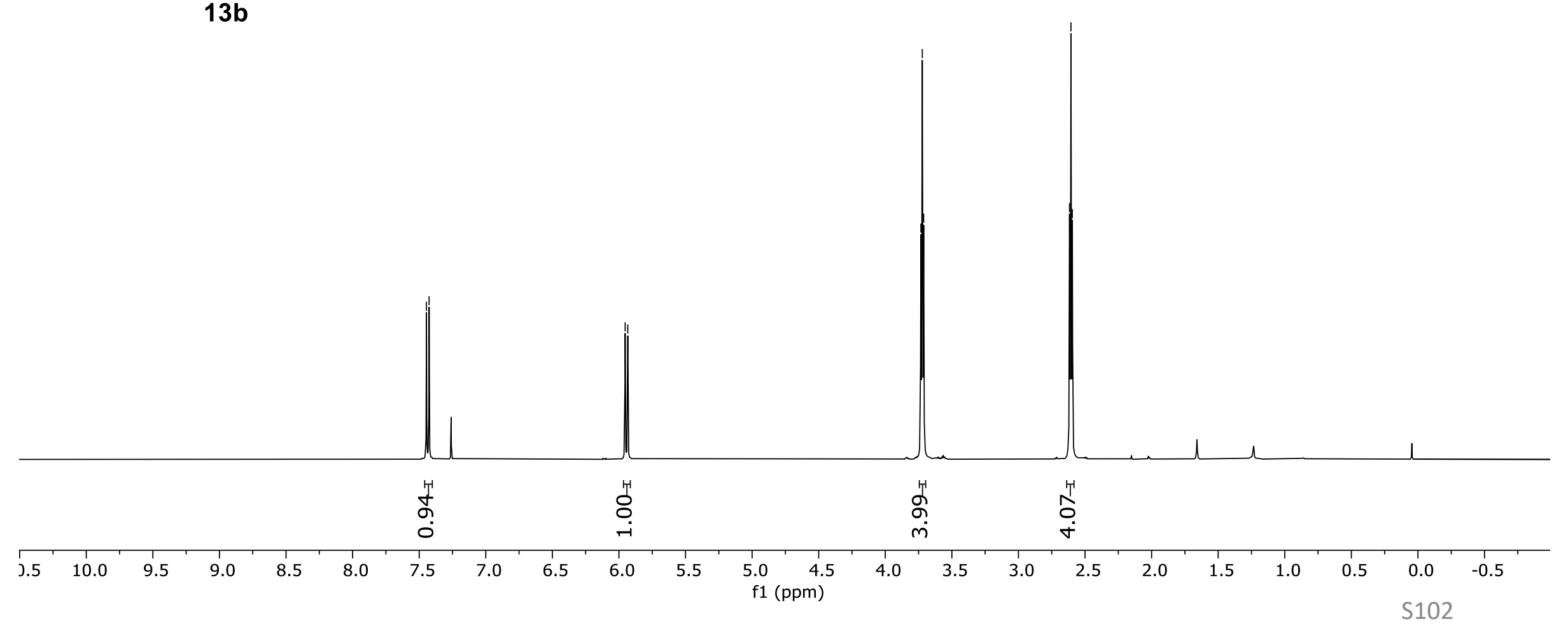




\section{$\mathrm{CDCl}_{3}, 151 \mathrm{MHz}$}

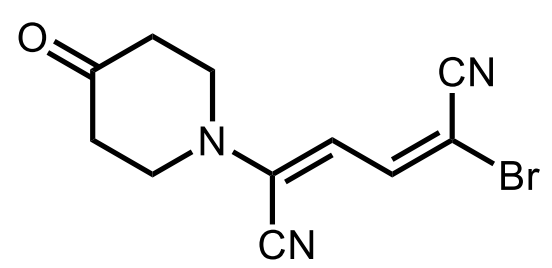

13b
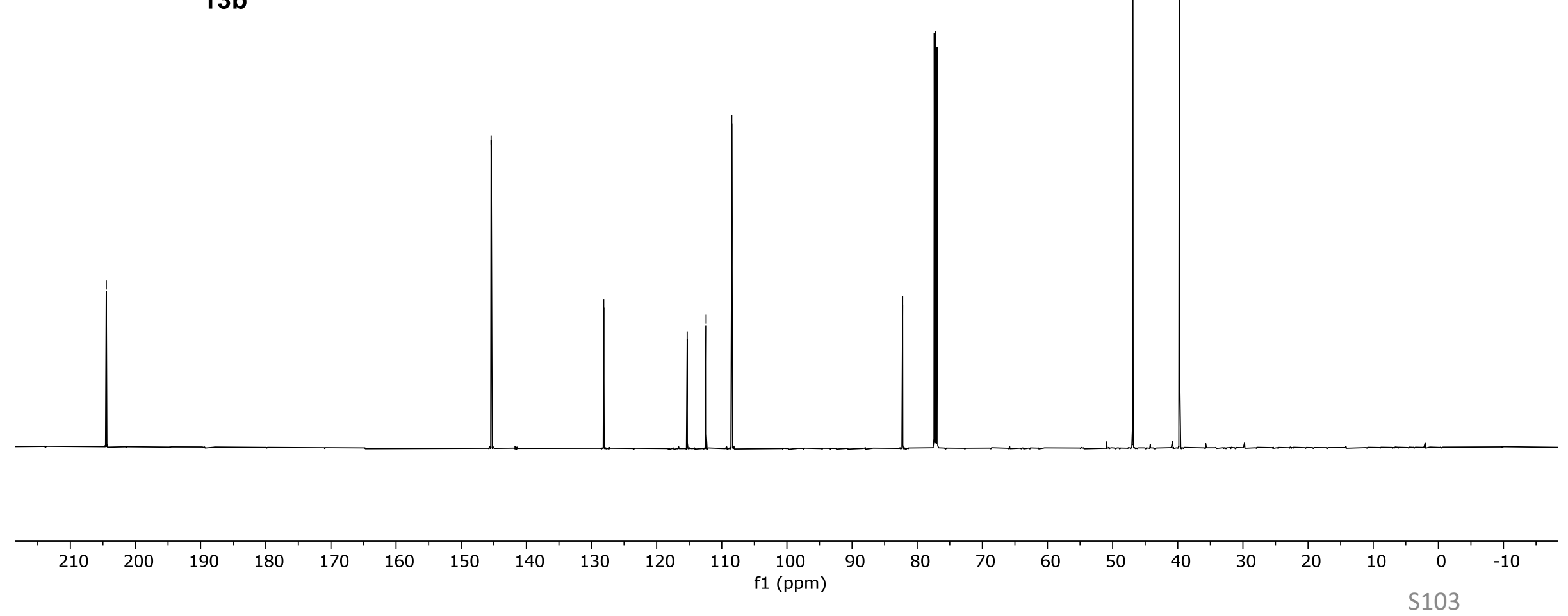


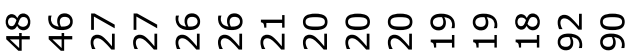

N N N N N N N N

ת

\section{$\mathrm{CDCl}_{3}, 600 \mathrm{MHz}$}

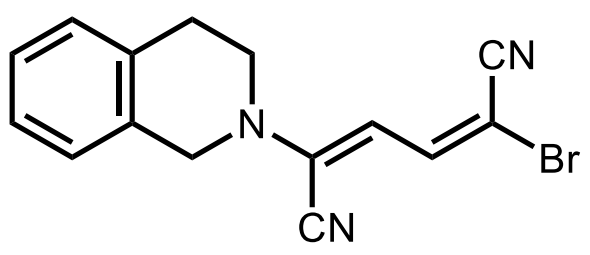

$14 b$

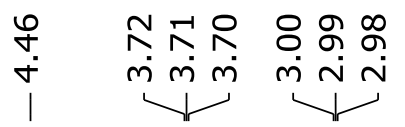
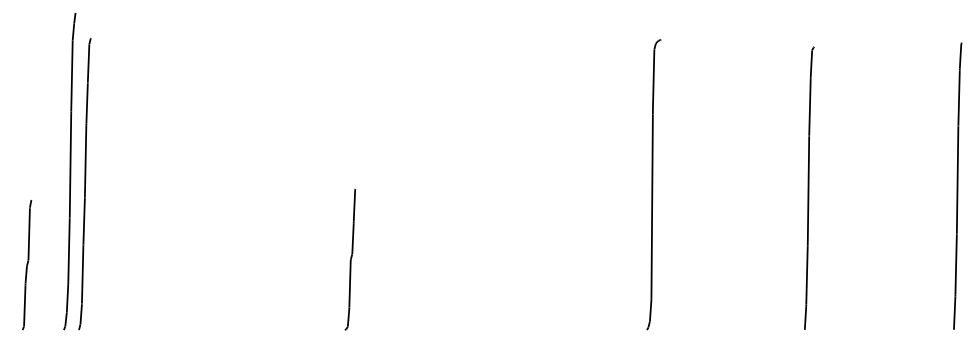

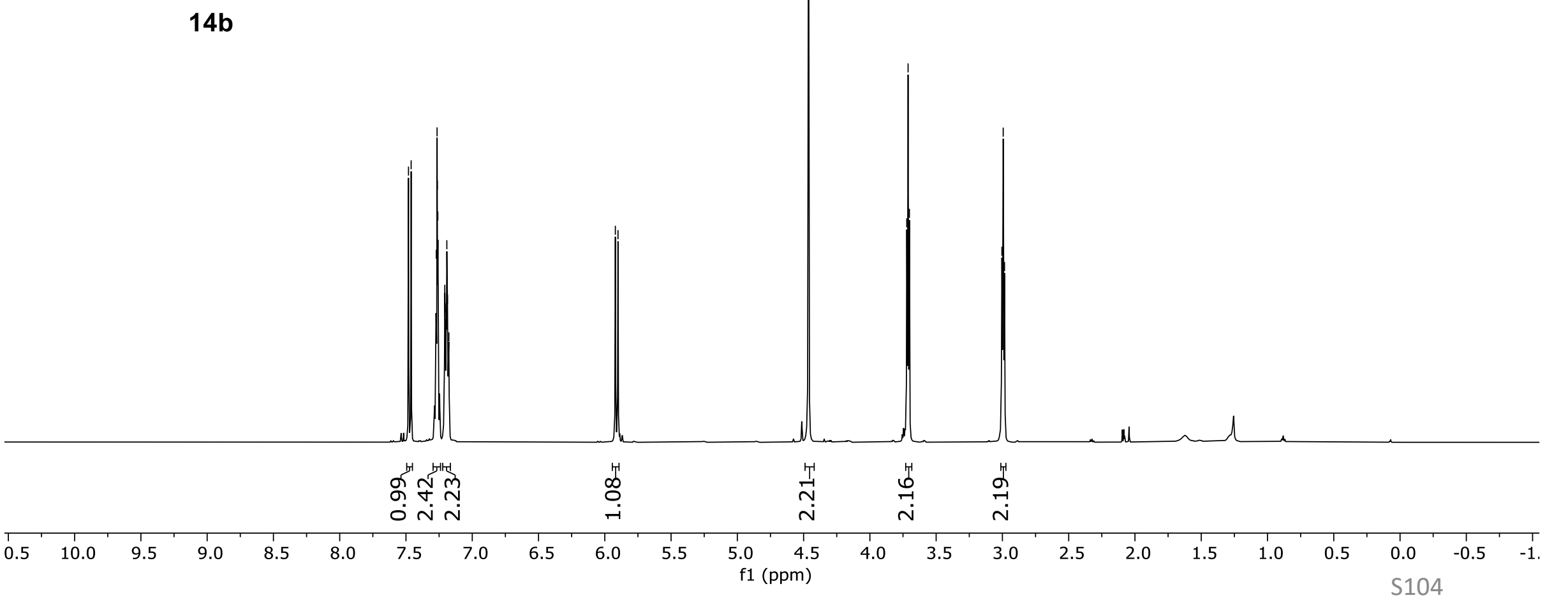




\section{$\mathrm{CDCl}_{3}, 151 \mathrm{MHz}$}<smiles>N#C/C(Br)=C\C=C(/C#N)N1CCc2ccccc2C1</smiles>

14b
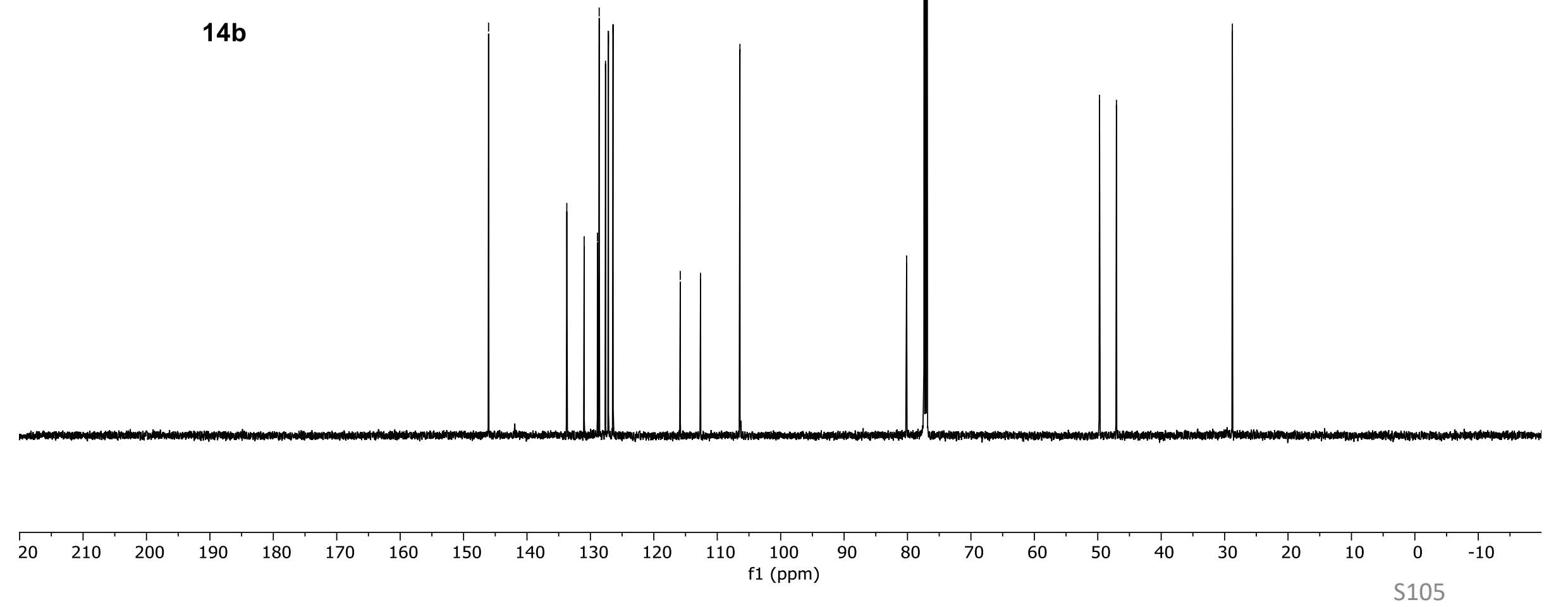


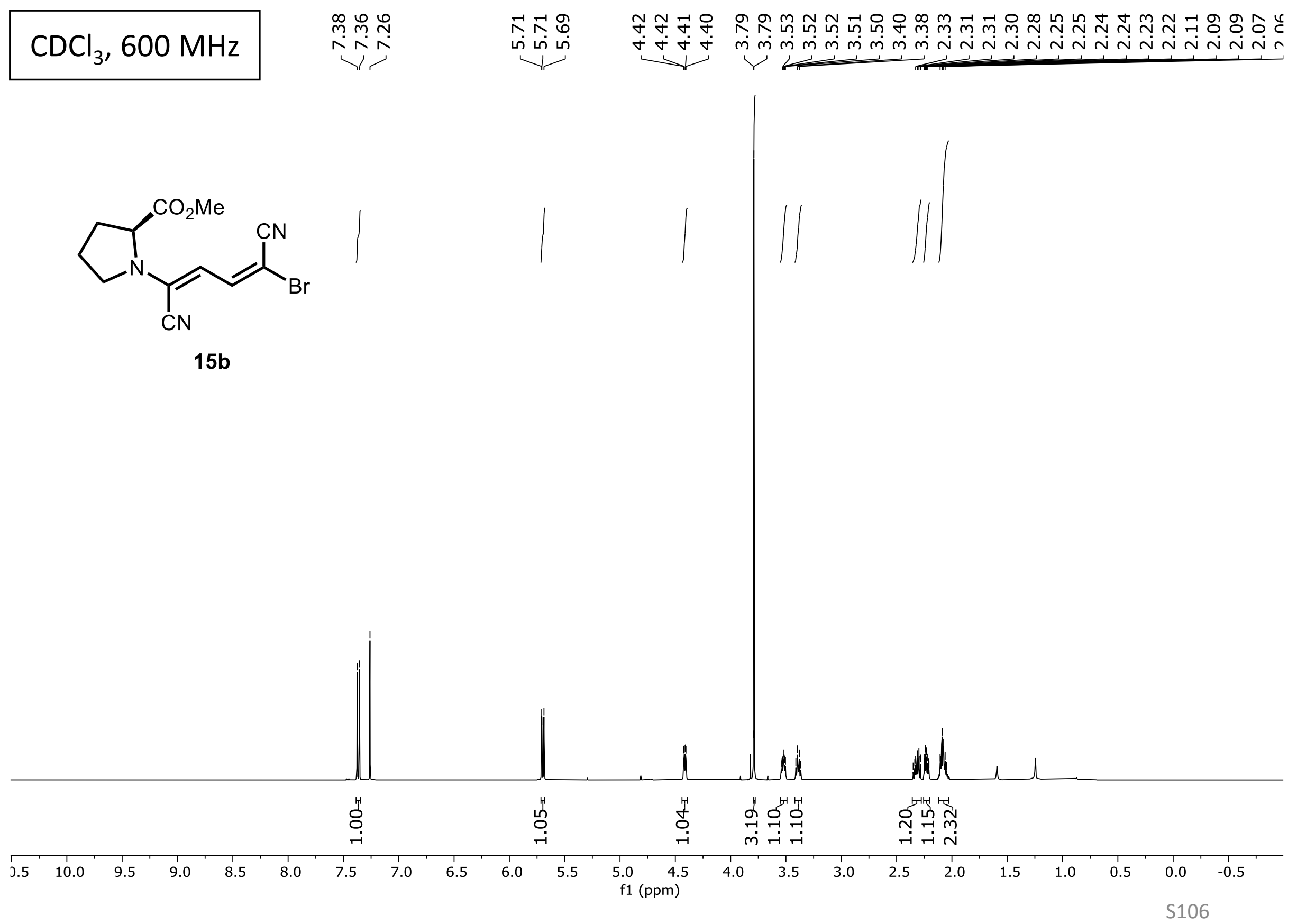



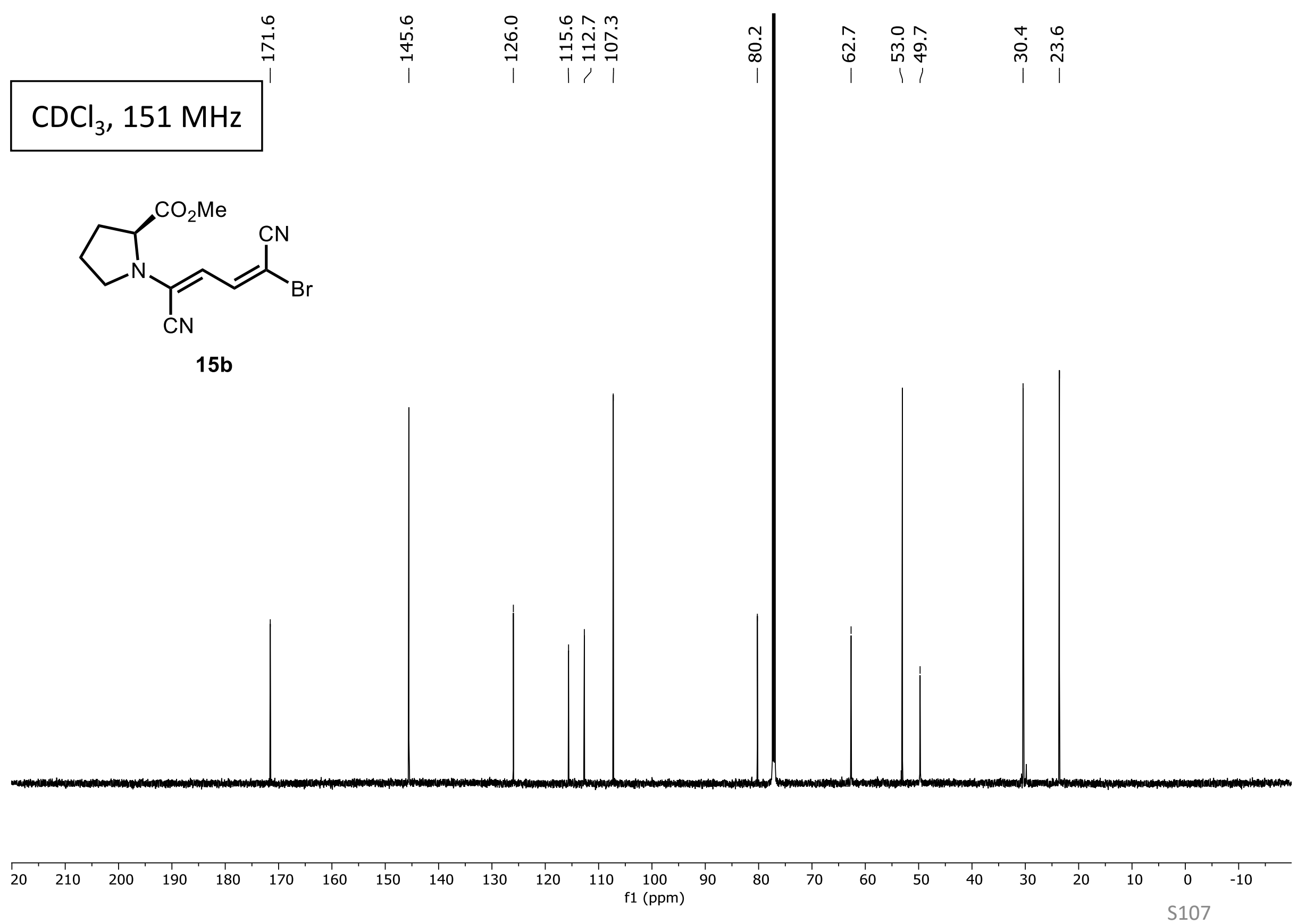


\section{$\mathrm{CDCl}_{3}, 600 \mathrm{MHz}$}
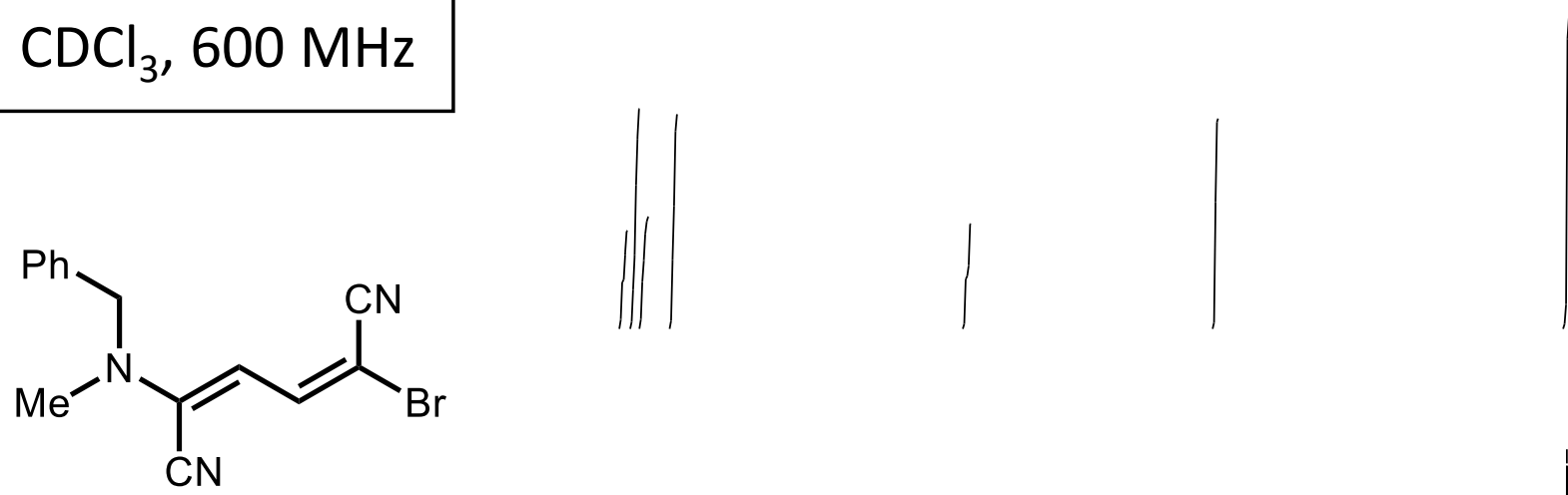

16b

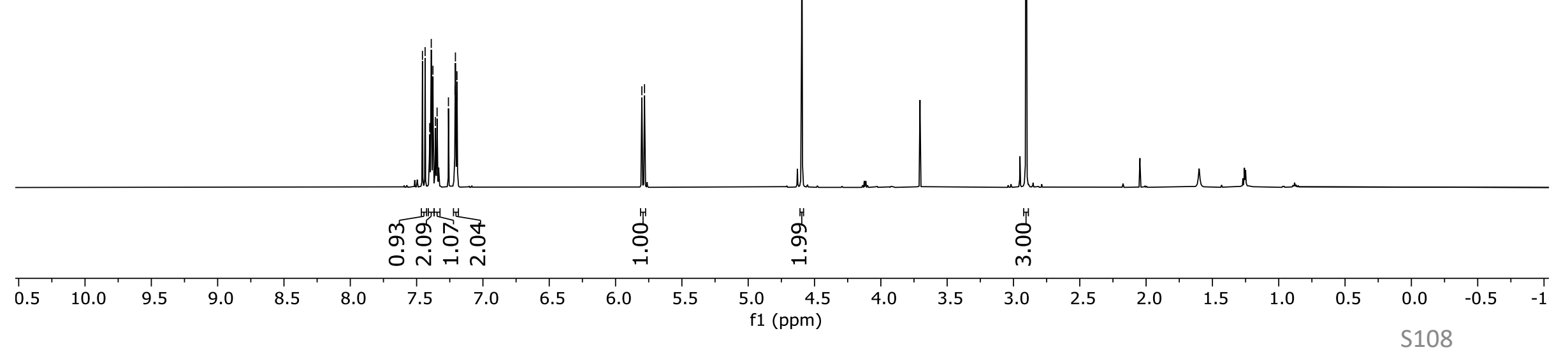




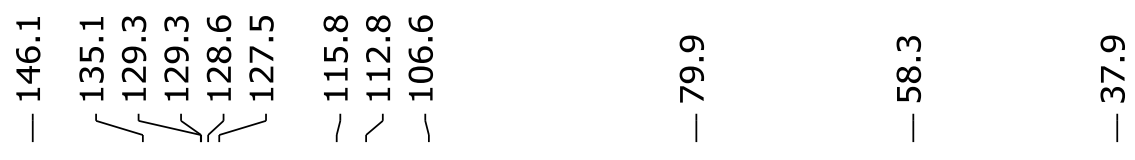

\section{$\mathrm{CDCl}_{3}, 151 \mathrm{MHz}$}

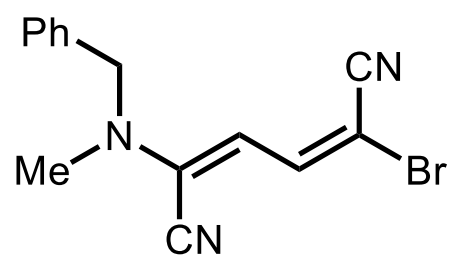

$16 b$
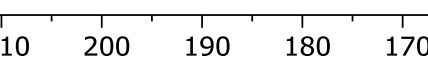

160
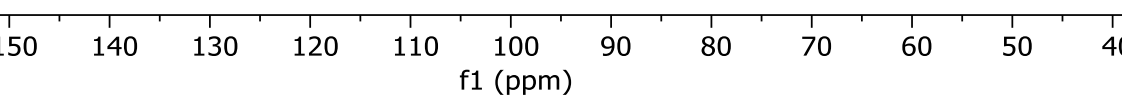


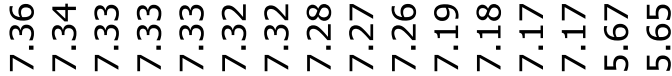

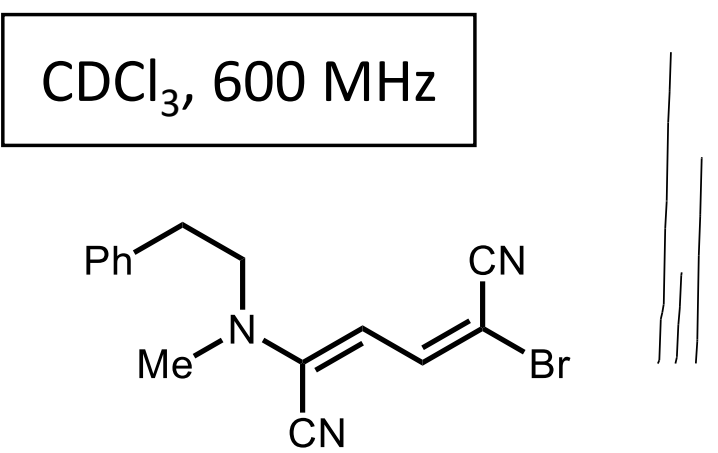

$17 b$

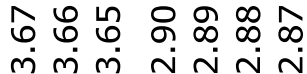

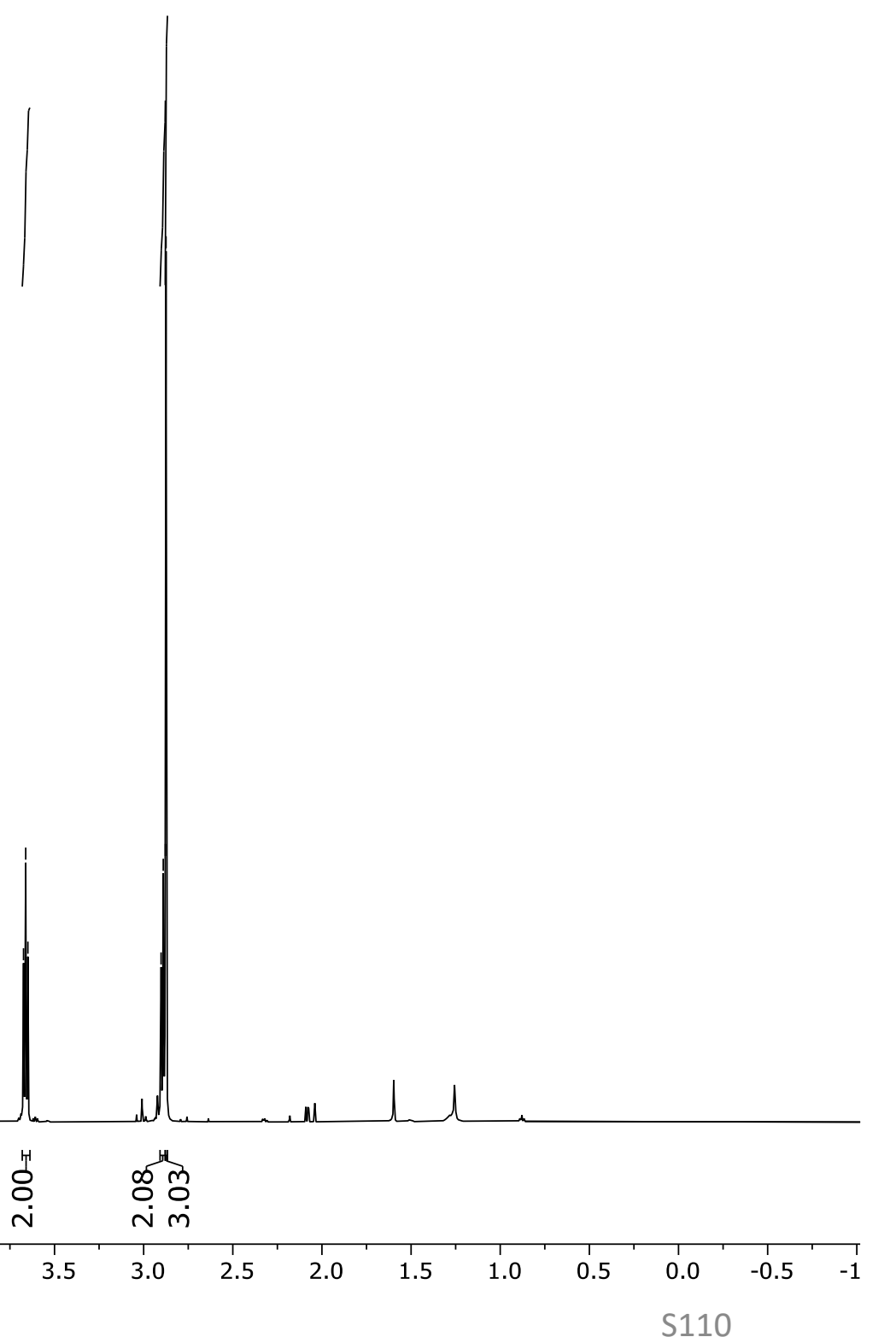




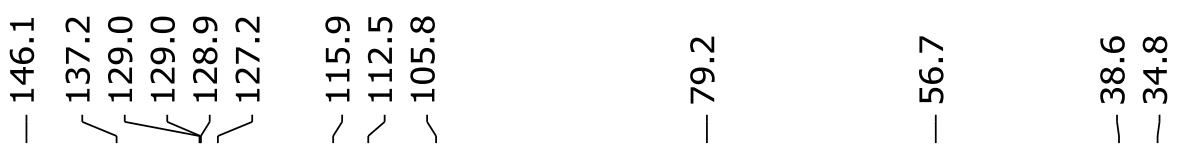

\section{$\mathrm{CDCl}_{3}, 151 \mathrm{MHz}$}

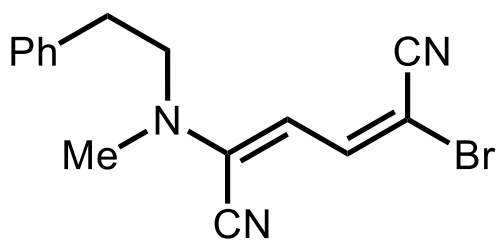

$17 b$

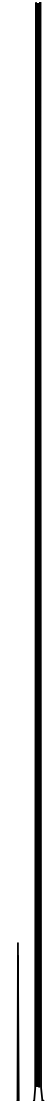

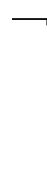
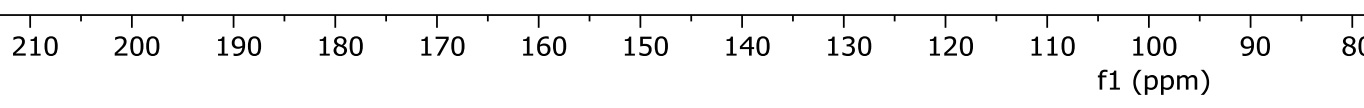

$70 \quad 60$
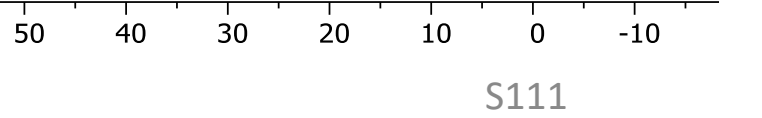


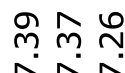

NN

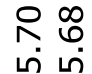

$\stackrel{n}{\text { ํㅜ }}$

\section{$\mathrm{CDCl}_{3}, 600 \mathrm{MHz}$}<smiles>CN(C)/C(C#N)=C/C=C(/Br)C#N</smiles>

$18 b$

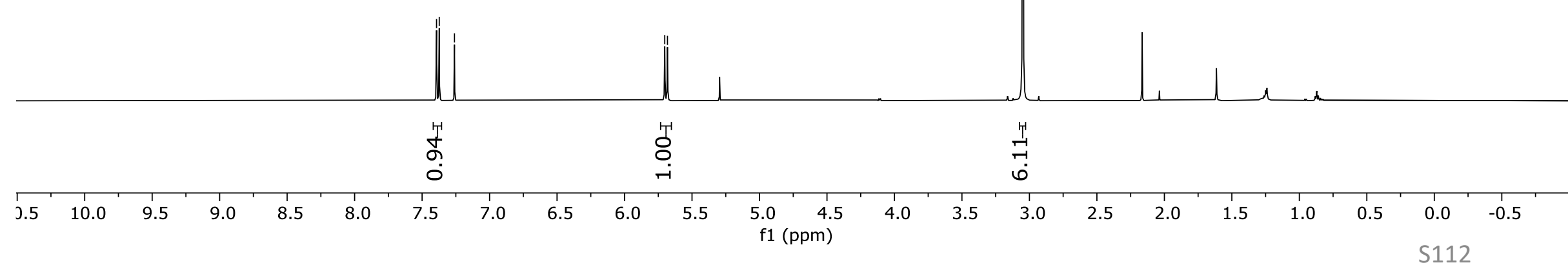




\section{$\mathrm{CDCl}_{3}, 151 \mathrm{MHz}$}<smiles>CN(C)/C(C#N)=C/C=C(\Br)C#N</smiles>

$18 b$

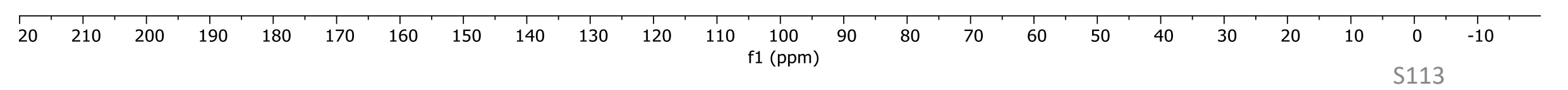




\section{$\mathrm{CDCl}_{3}, 600 \mathrm{MHz}$}
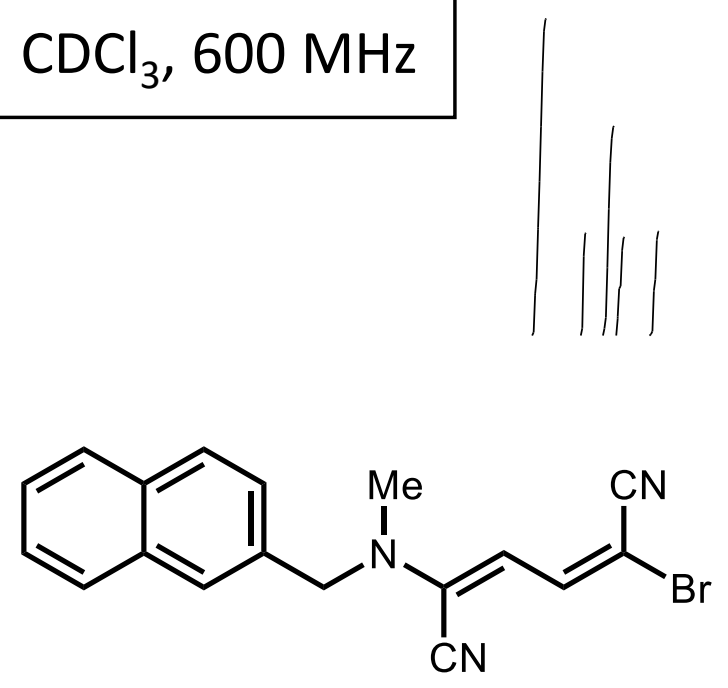

$19 b$

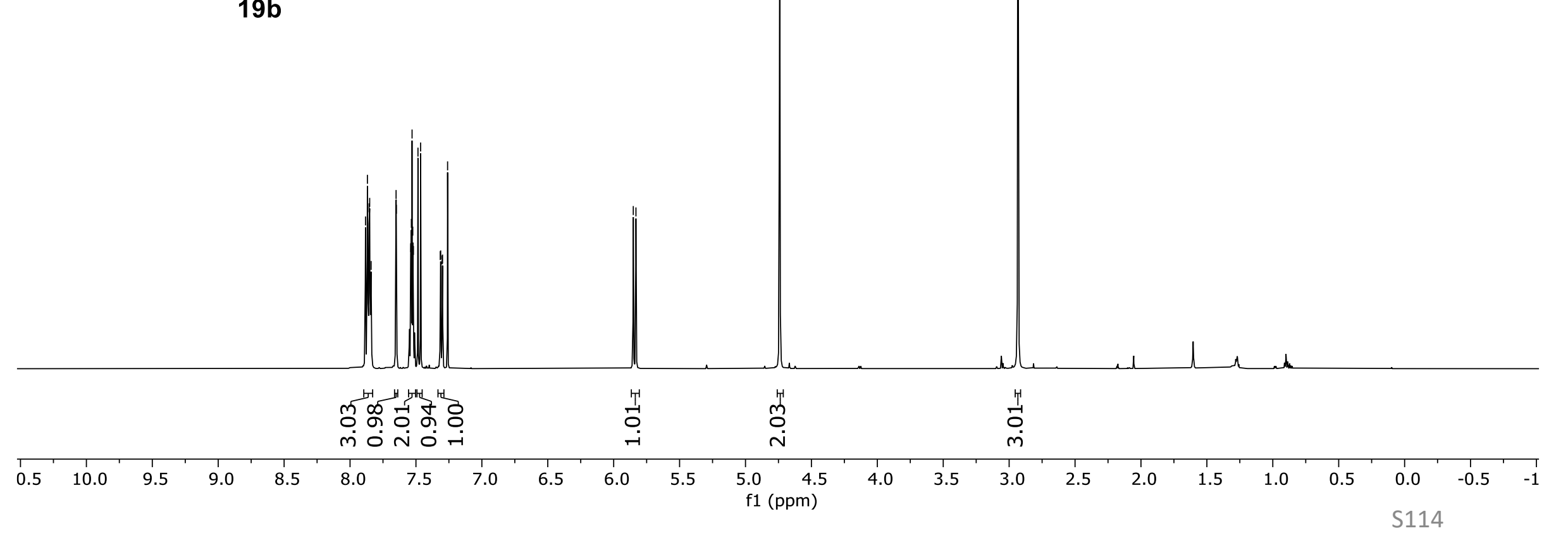




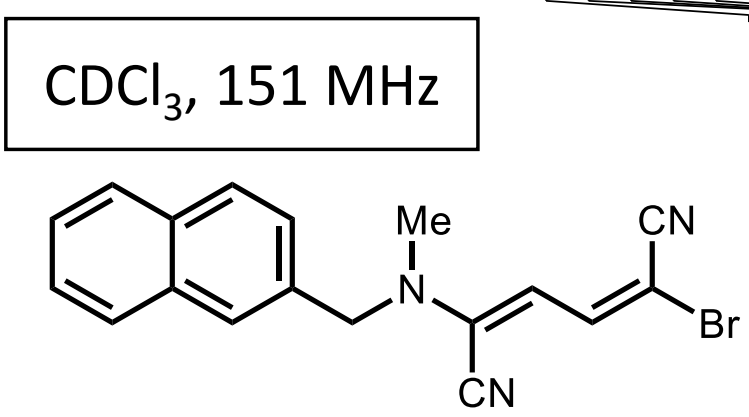

$19 b$

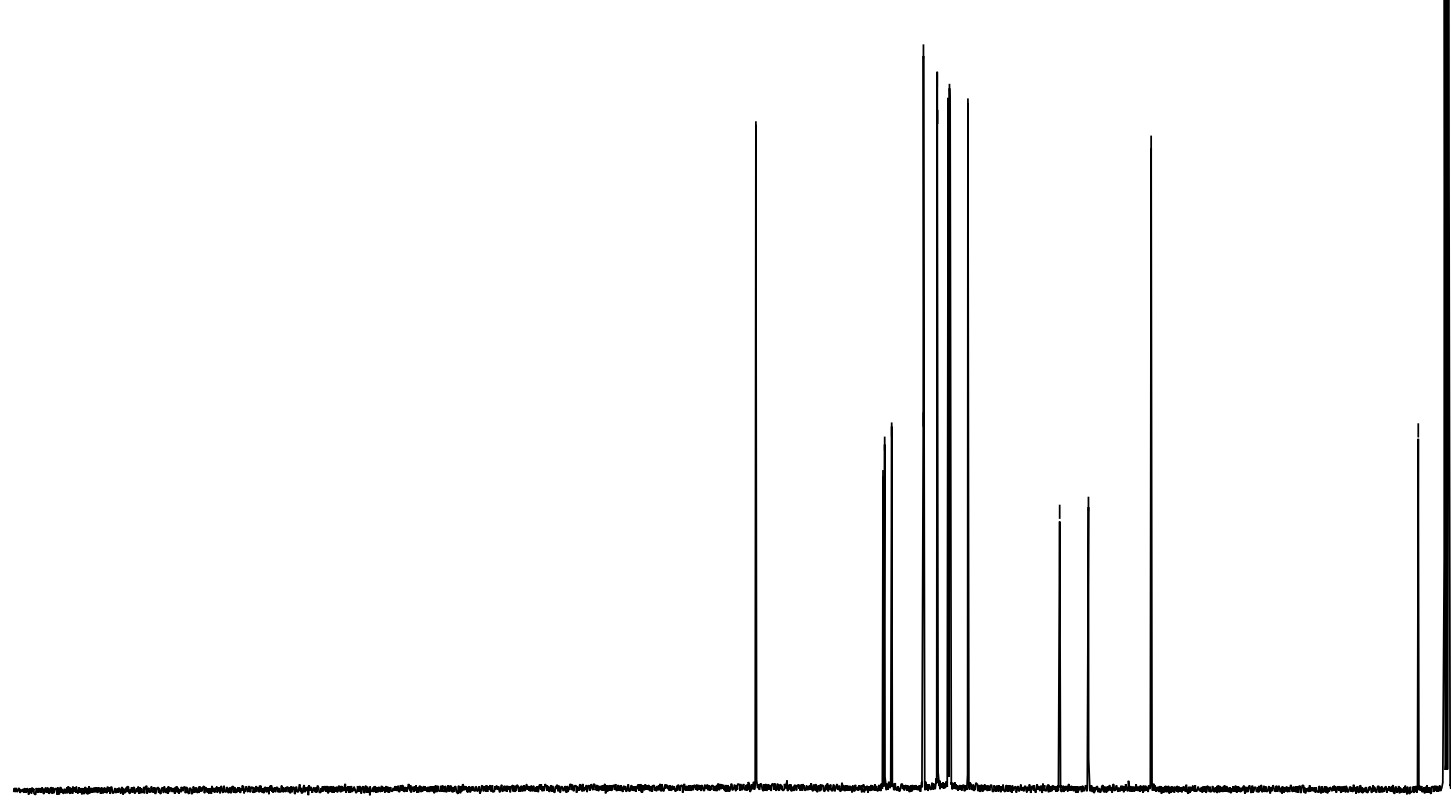




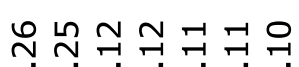

NNNNNN

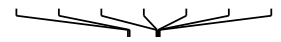

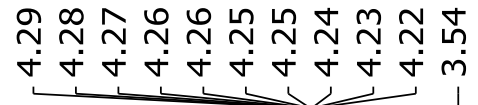

సָำ

각

\section{$\mathrm{CDCl}_{3}, 600 \mathrm{MHz}$}
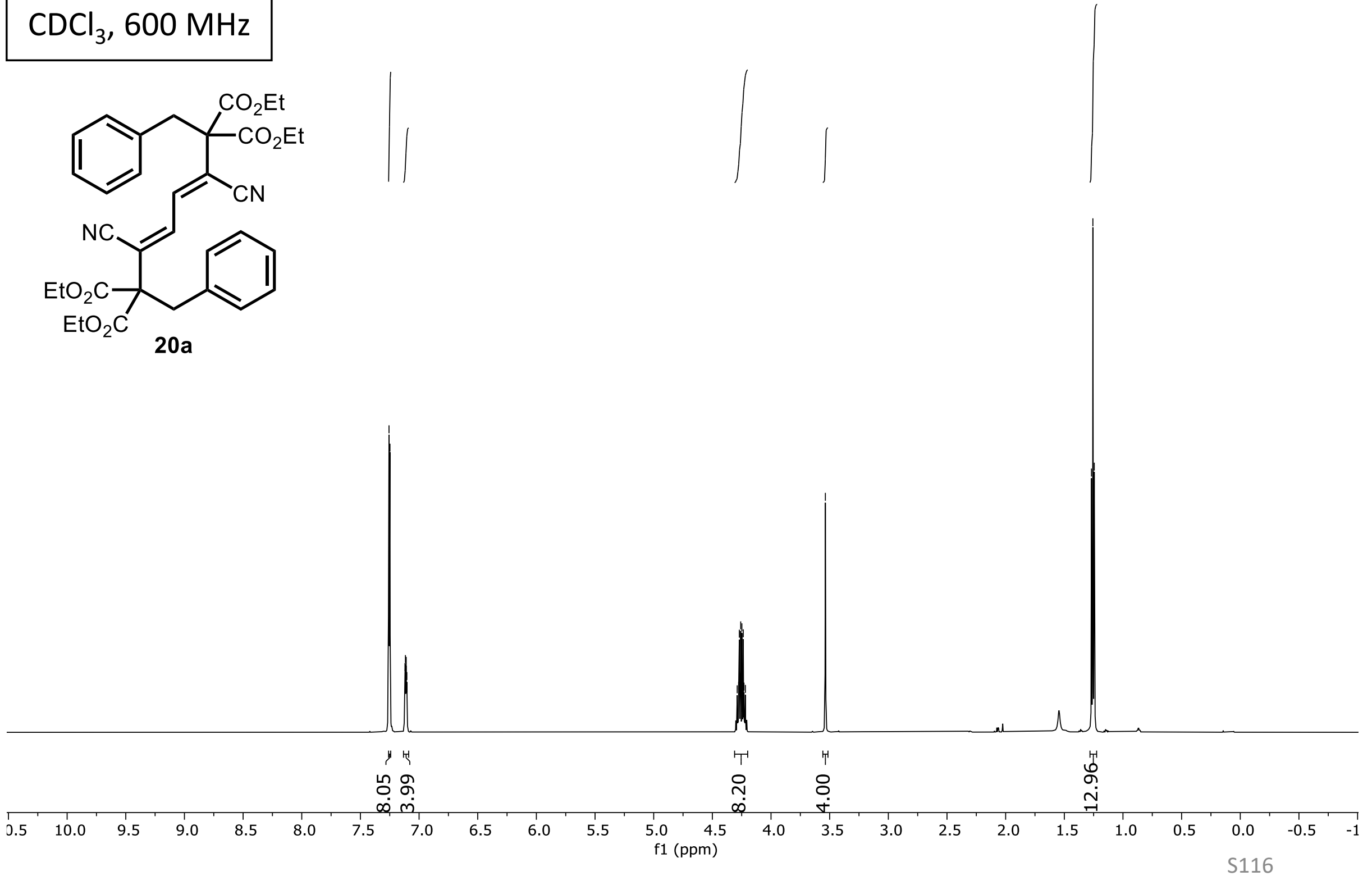

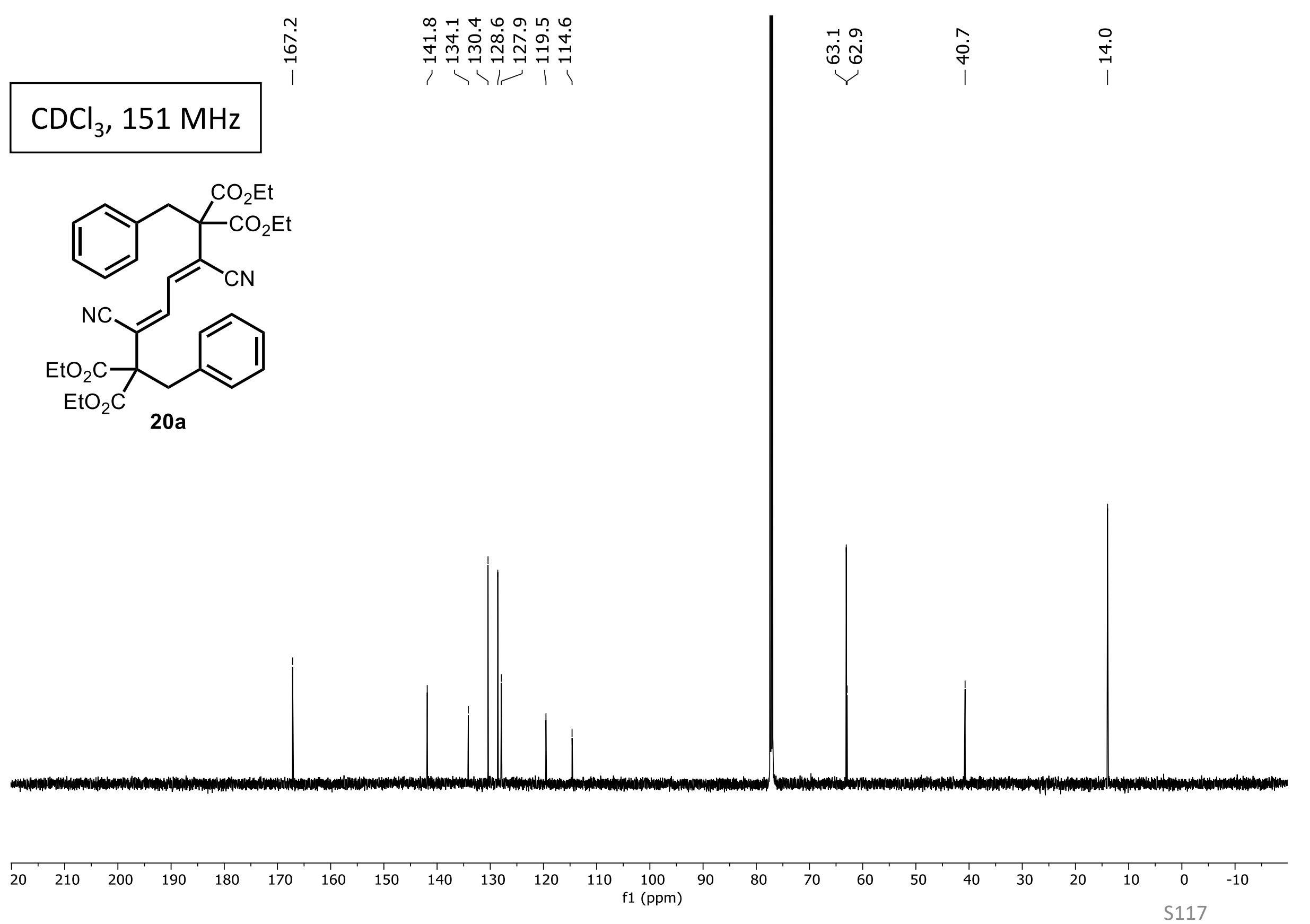


\section{$\mathrm{CDCl}_{3}, 600 \mathrm{MHz}$}
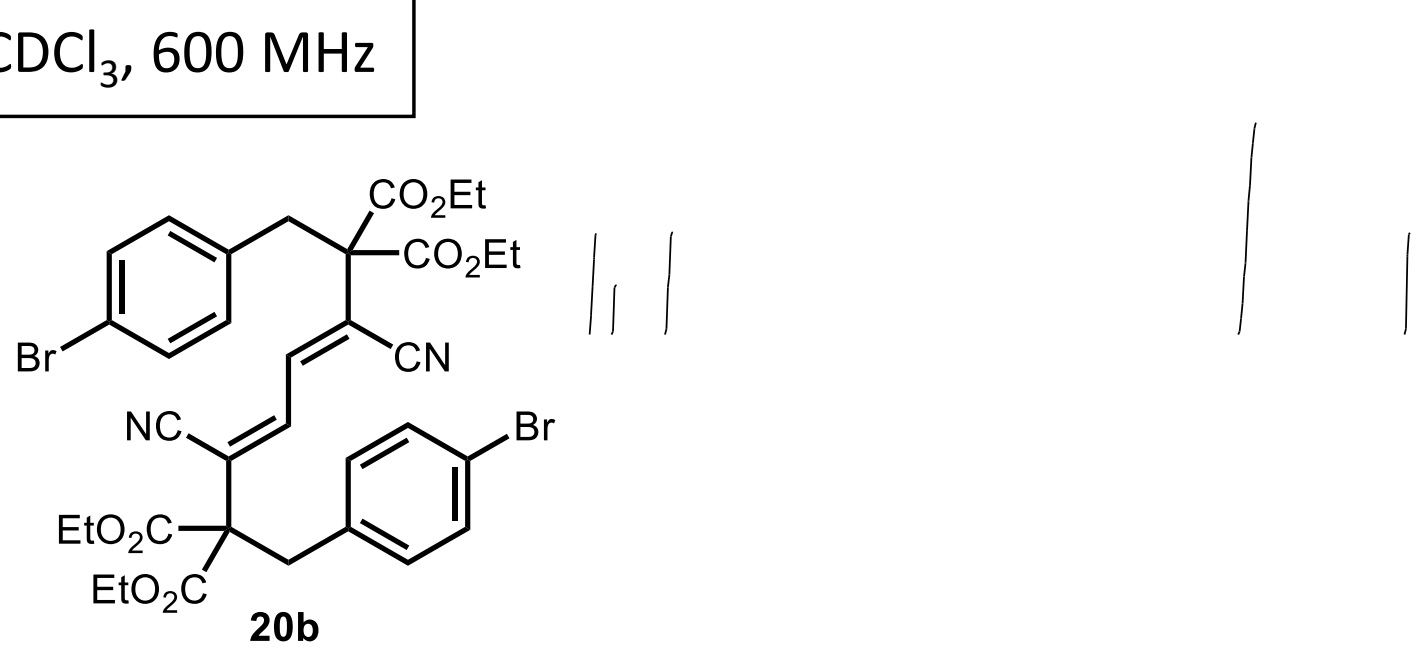

可 

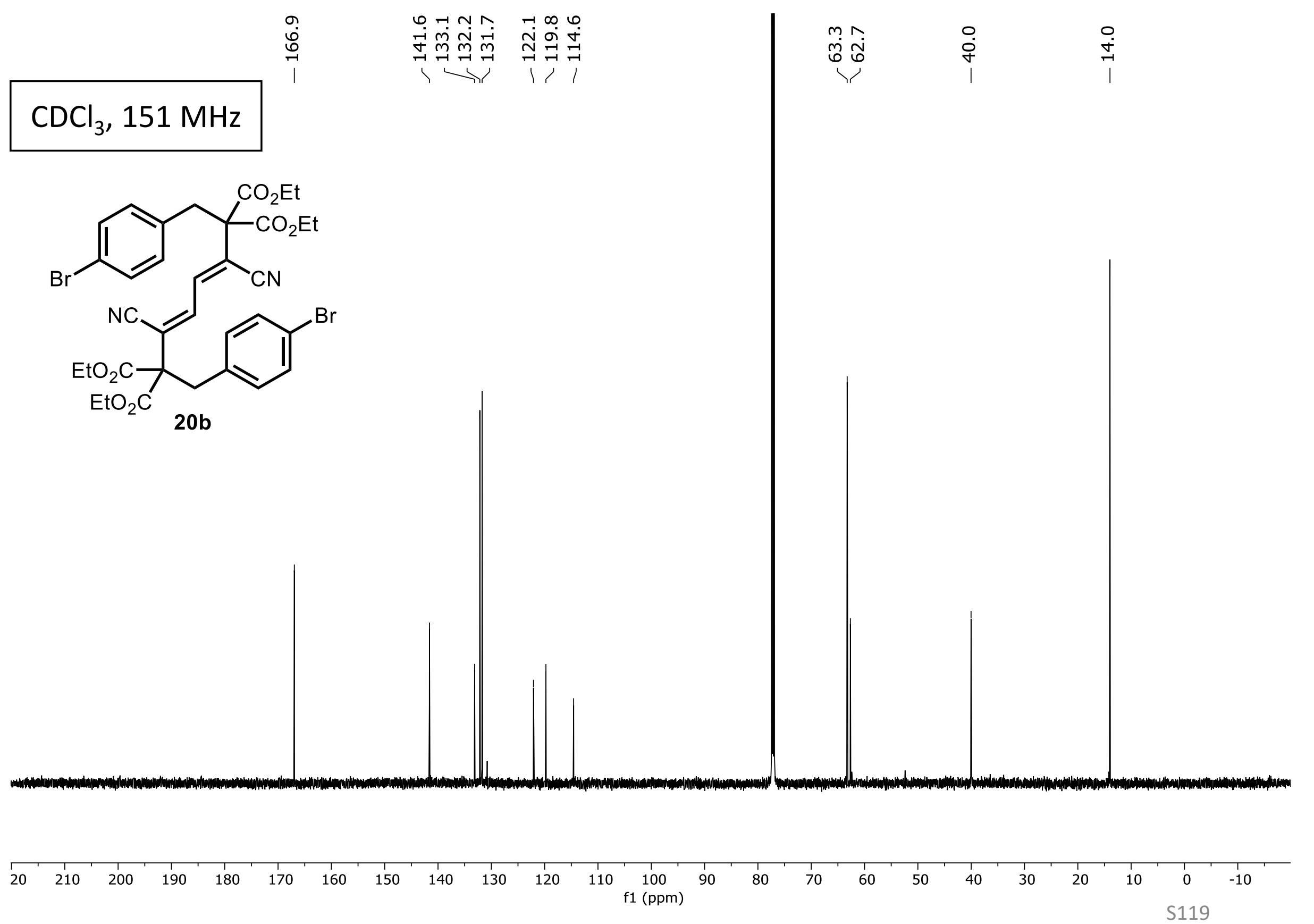


\section{$\mathrm{CDCl}_{3}, 600 \mathrm{MHz}$}
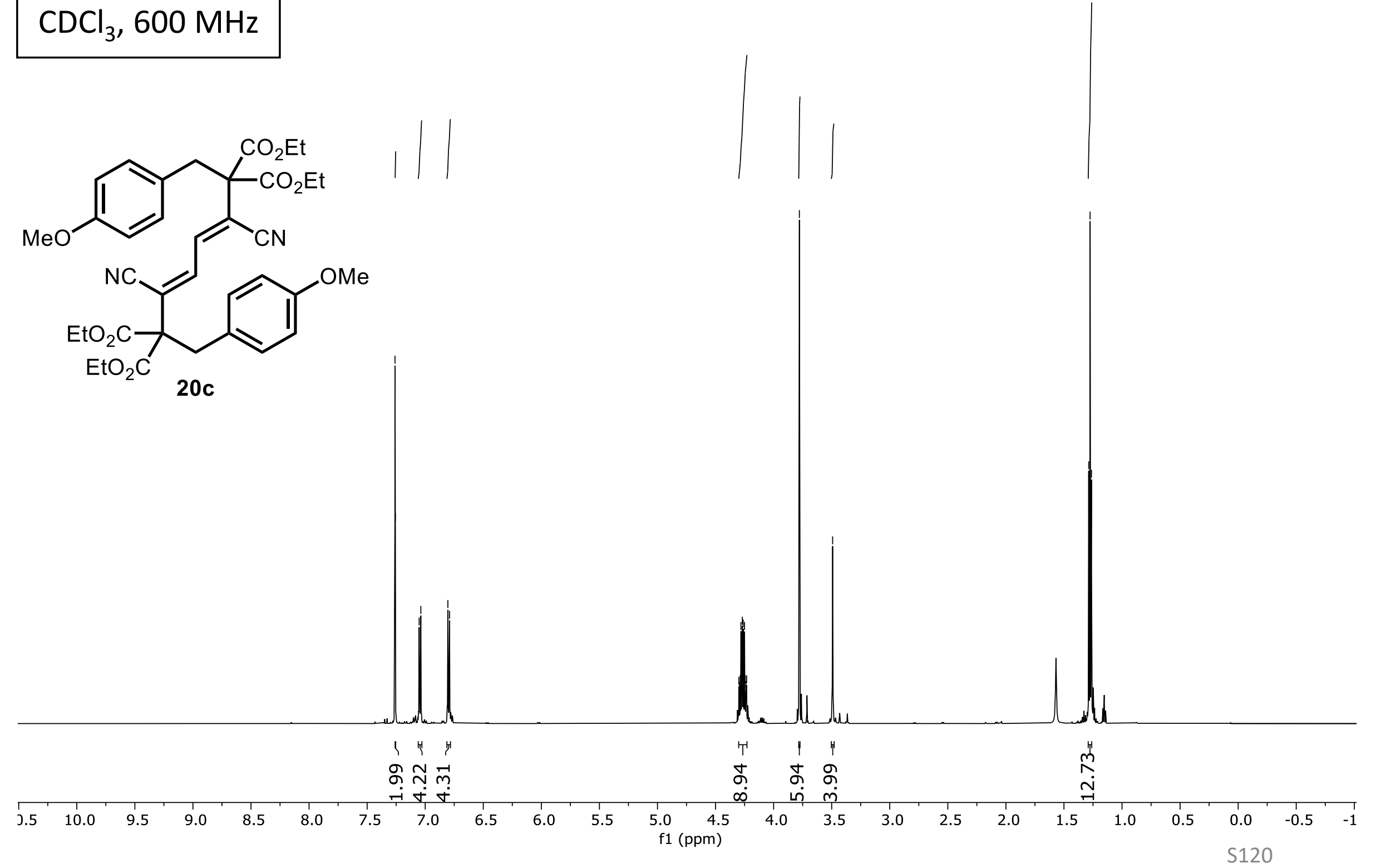


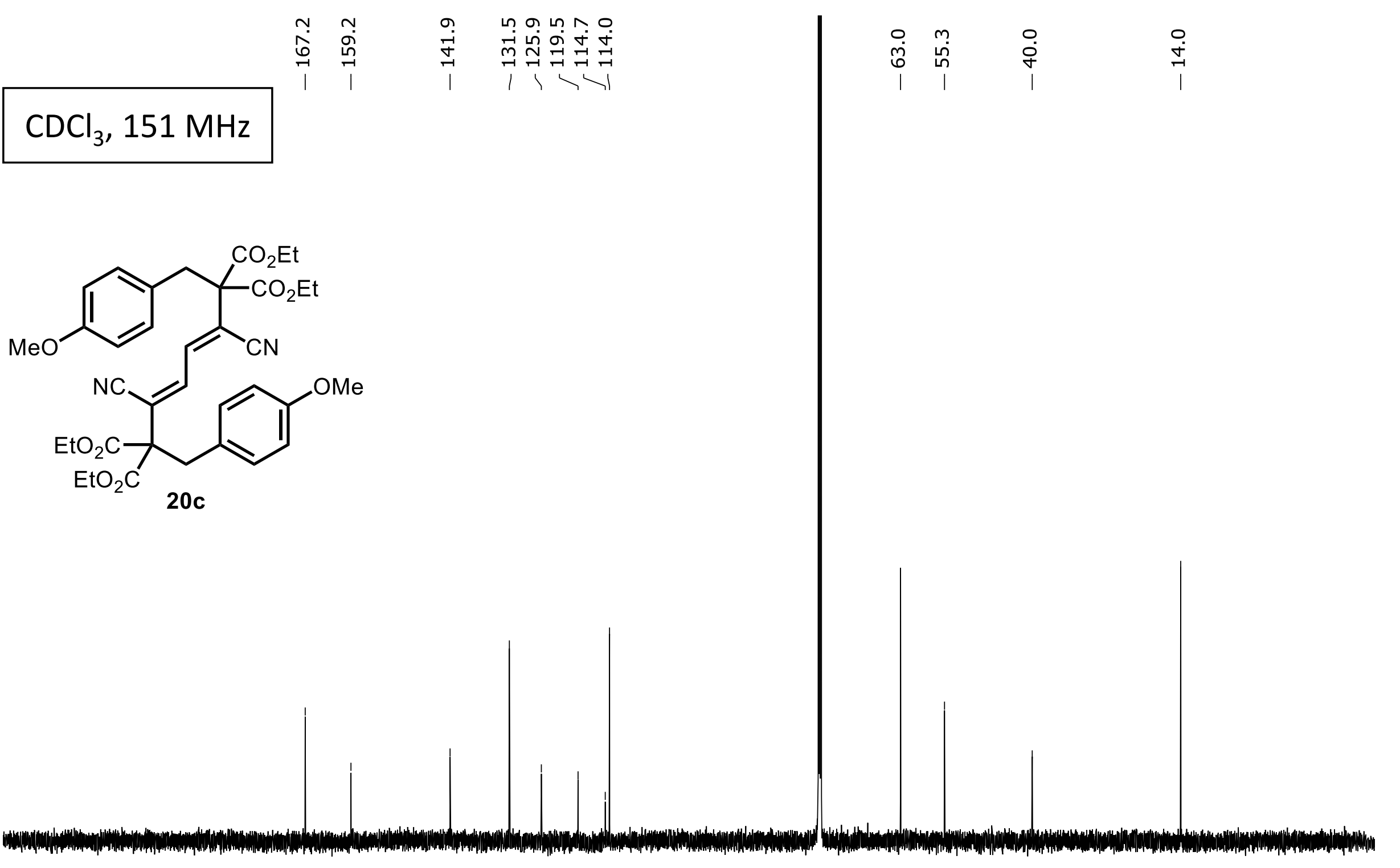




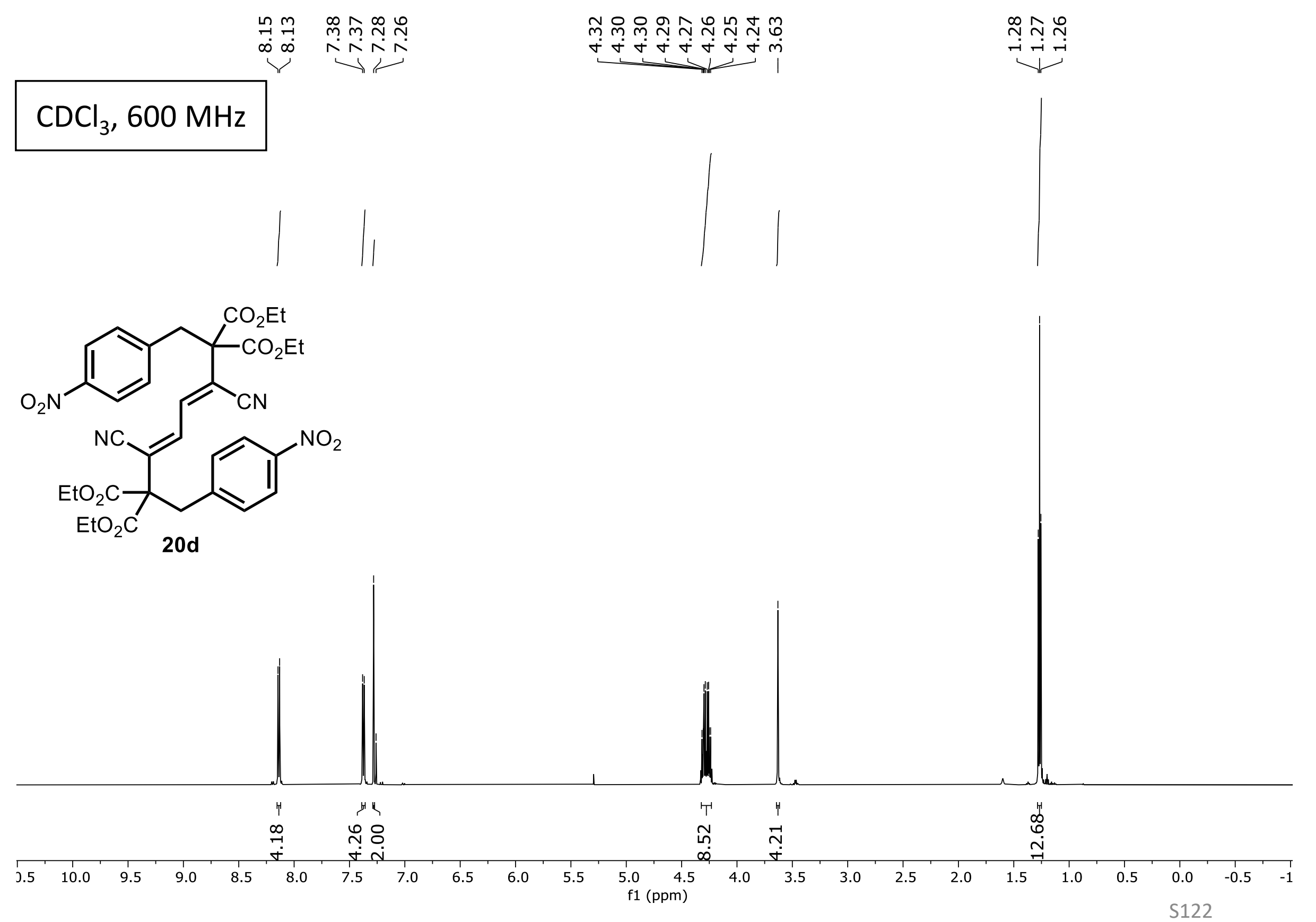



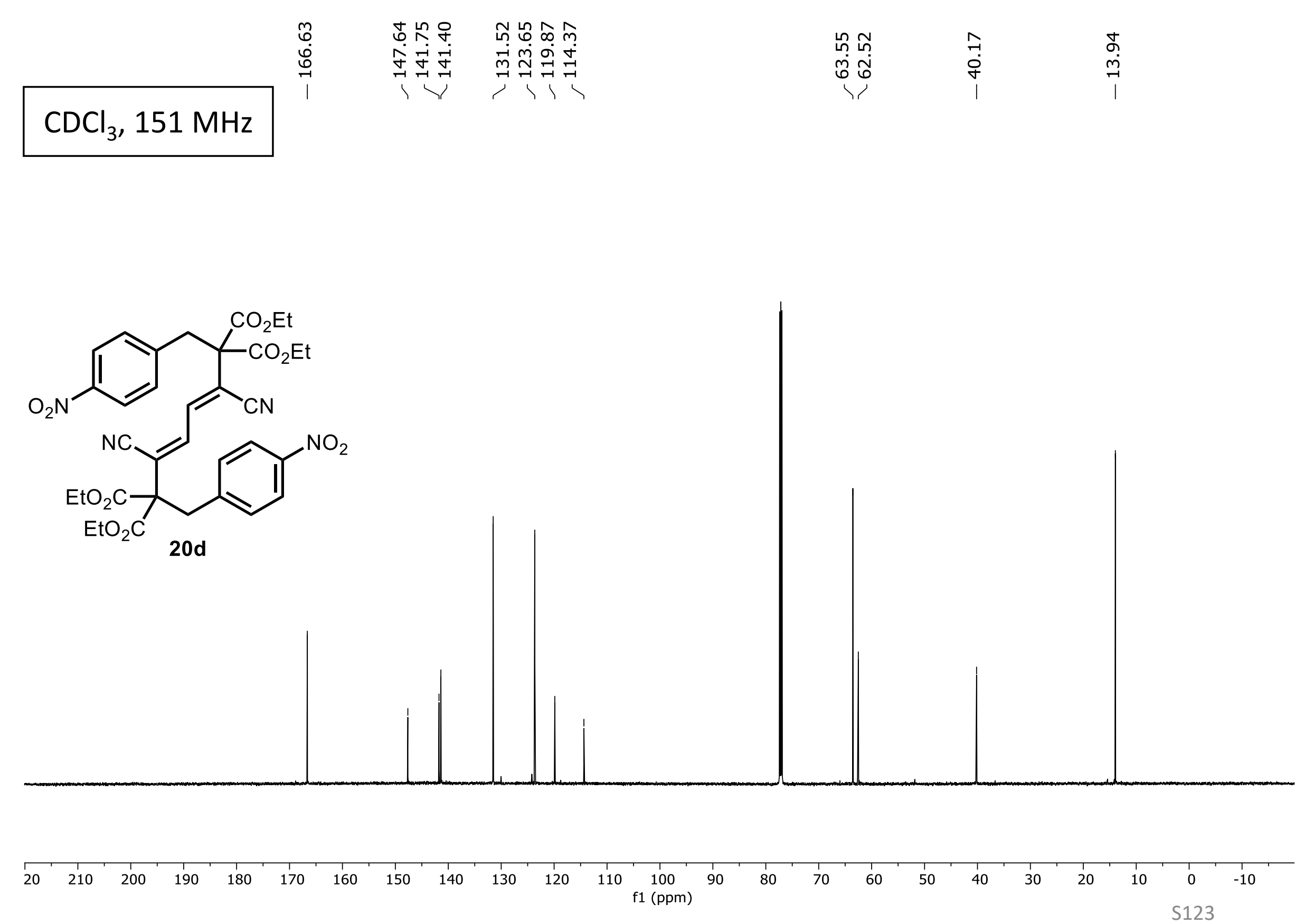


\section{$\mathrm{CDCl}_{3}, 600 \mathrm{MHz}$}<smiles>CCOCC(COCC)(COCC)/C(C#N)=C/C=C(\C#N)C(Cc1ccc(C)cc1)(Cc1ccc(C)cc1)C(=O)OCC</smiles>

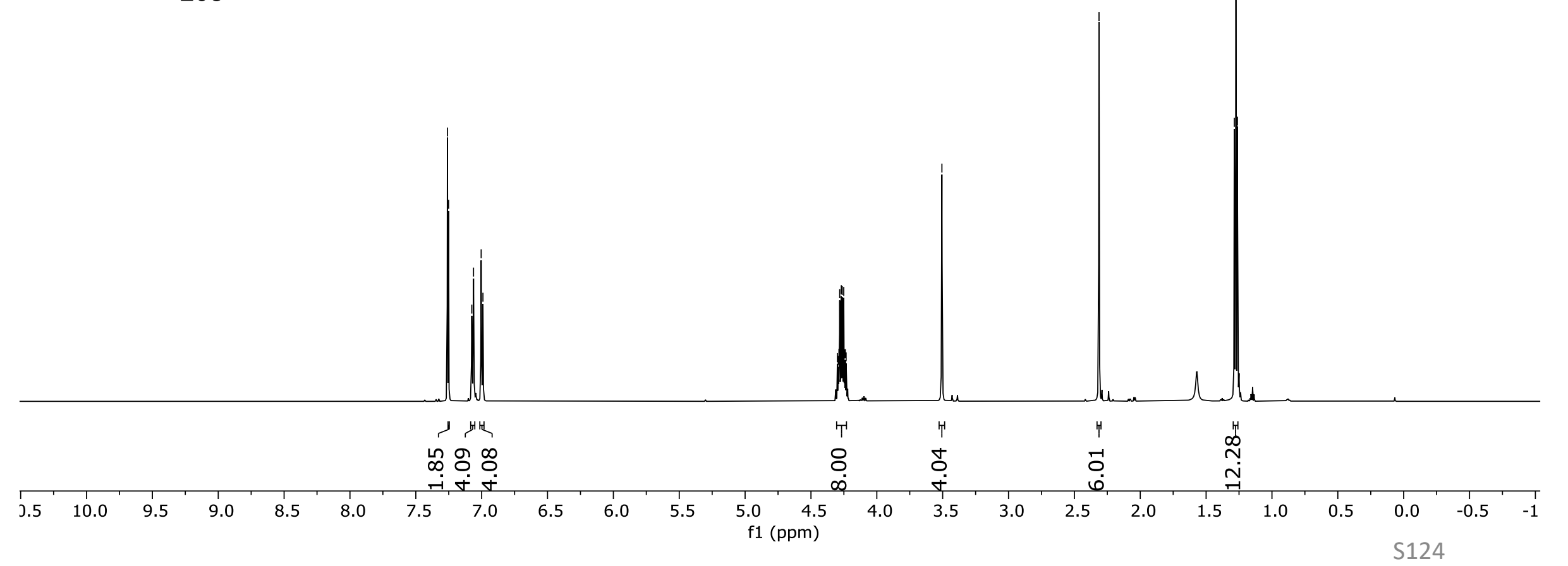




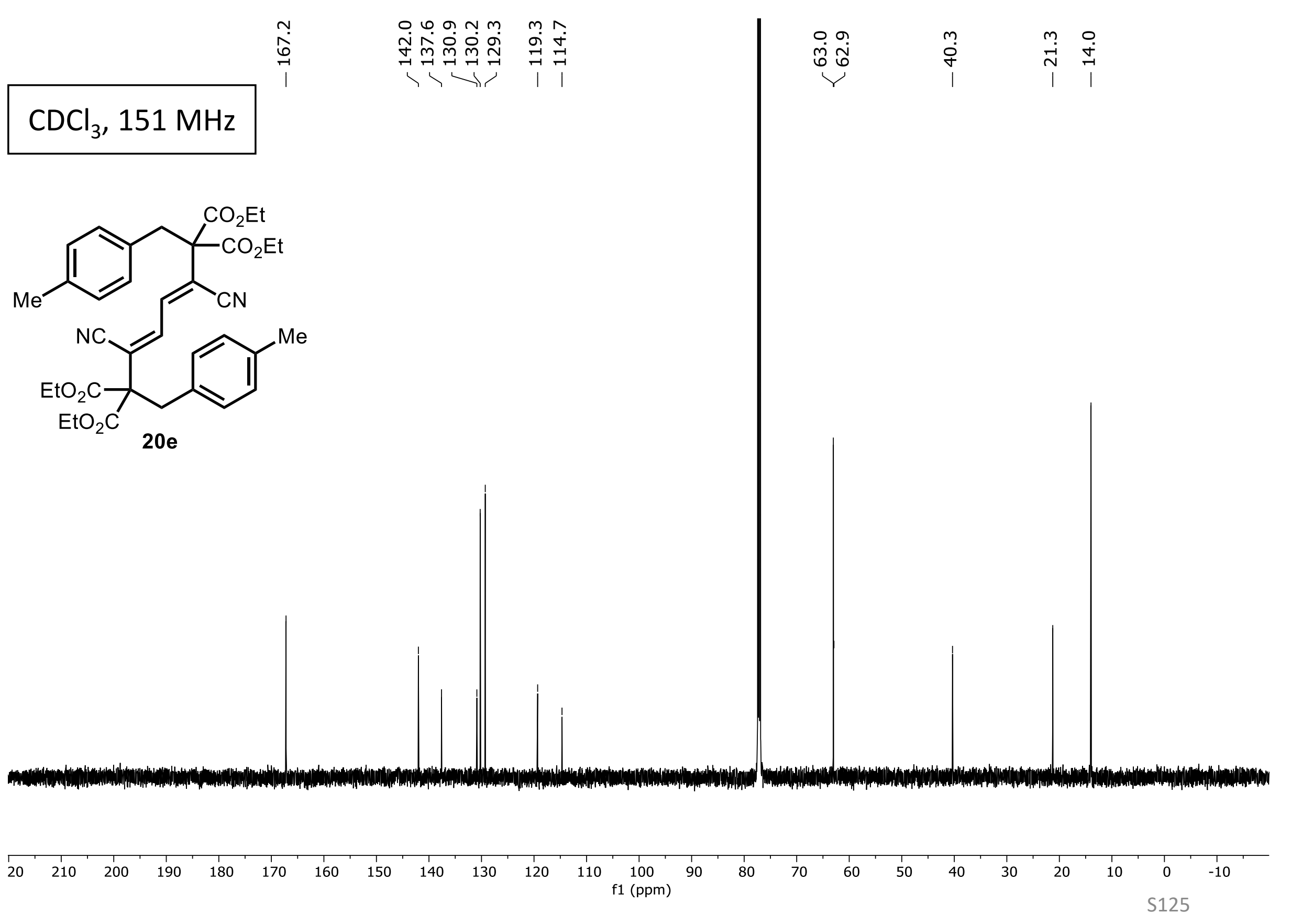




\section{$\mathrm{CDCl}_{3}, 600 \mathrm{MHz}$}

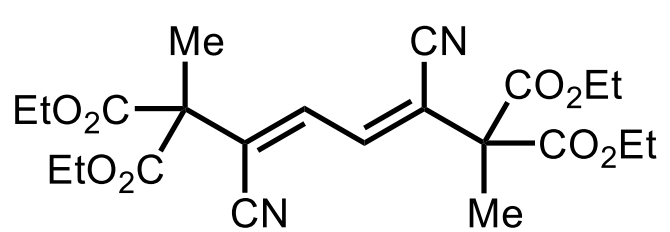

21

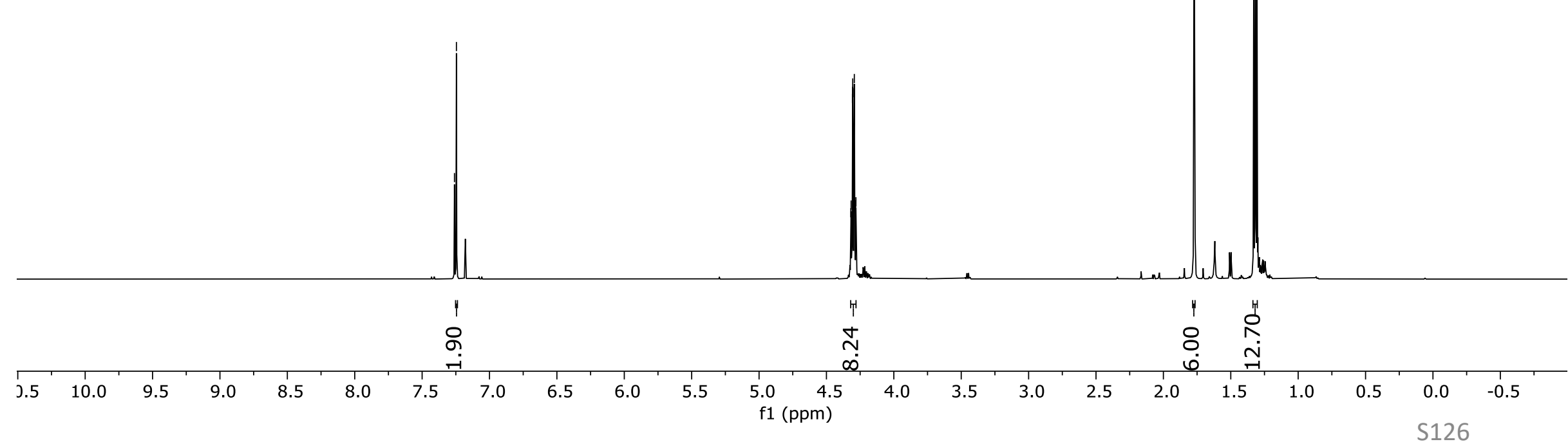




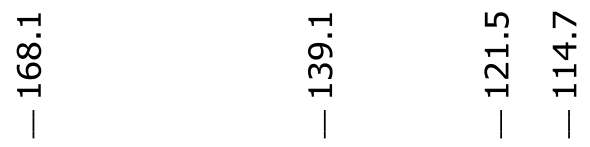

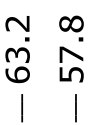
$\begin{array}{ll}0 \\ \stackrel{0}{\dot{*}} & \stackrel{+}{1} \\ 1 & 1\end{array}$
$\mathrm{CDCl}_{3}, 151 \mathrm{MHz}$

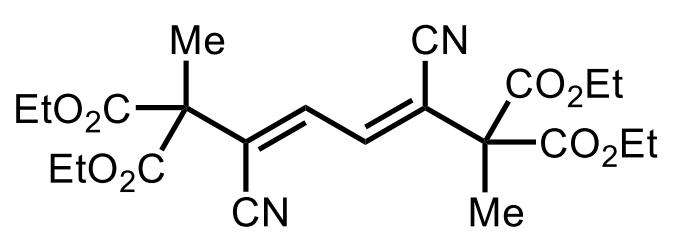

21
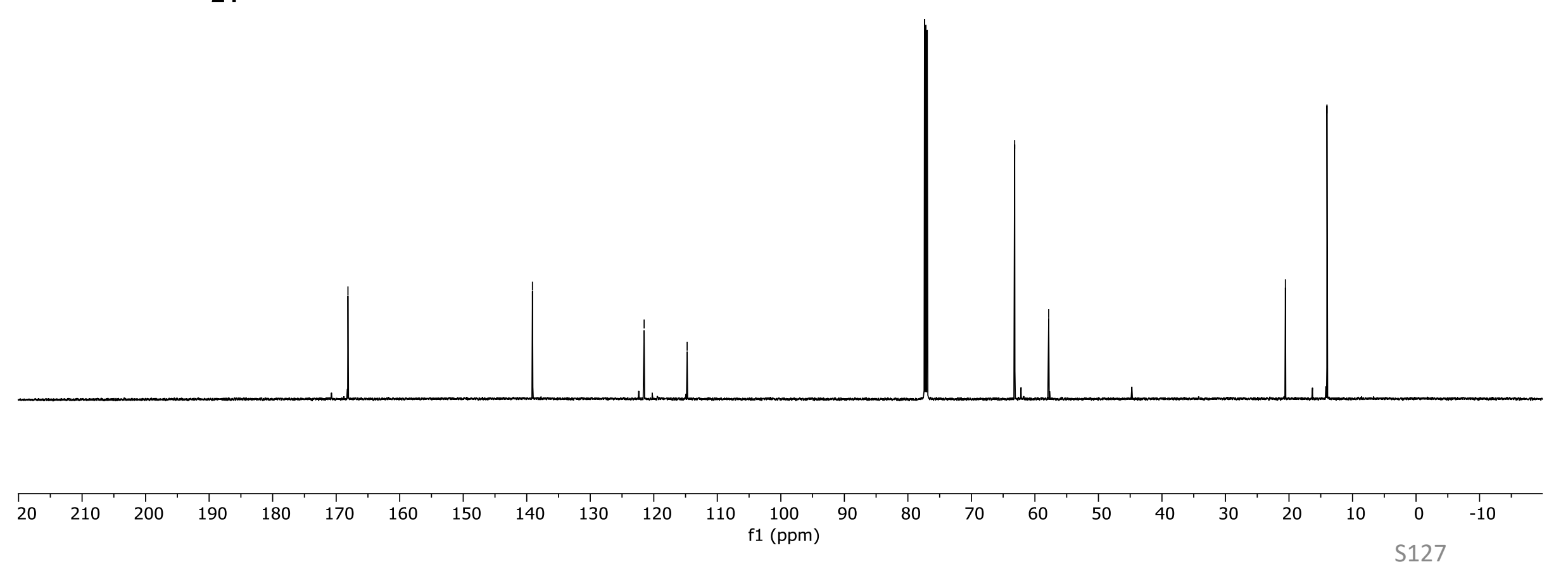


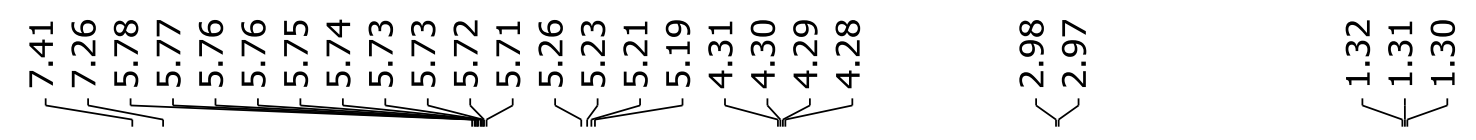

\section{$\mathrm{CDCl}_{3}, 600 \mathrm{MHz}$}
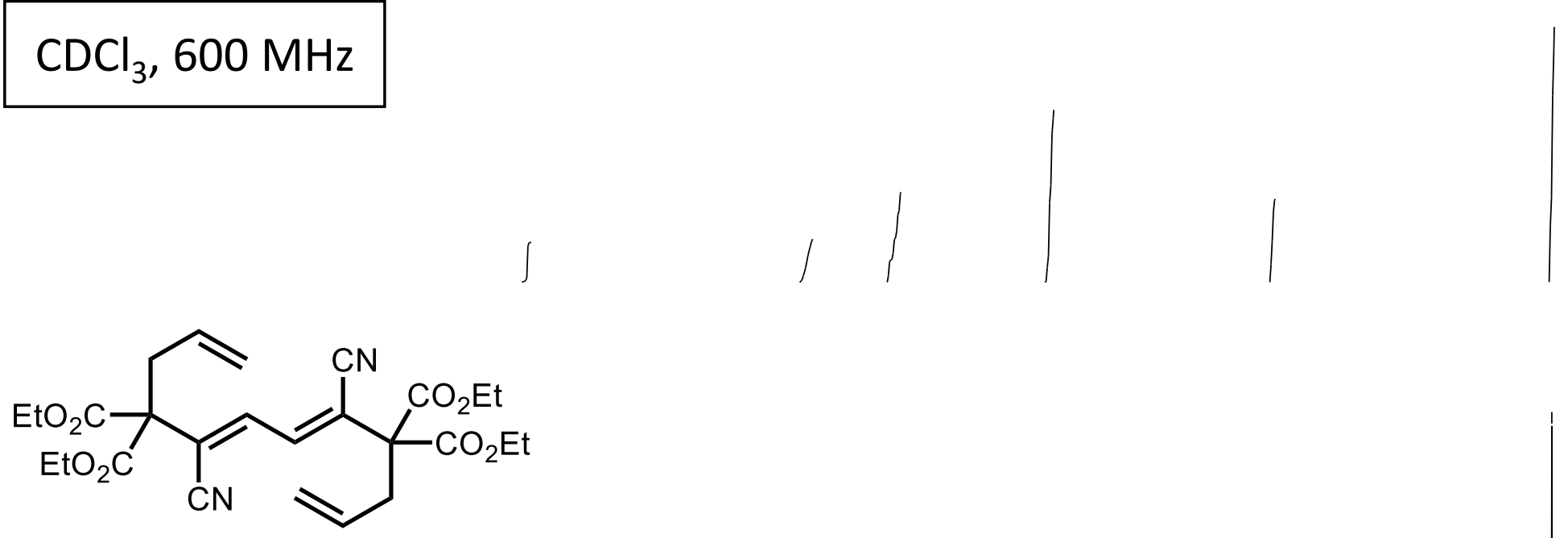

22

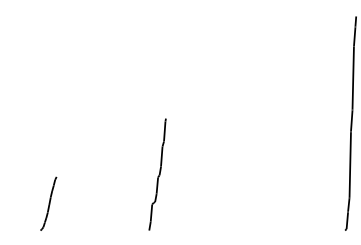




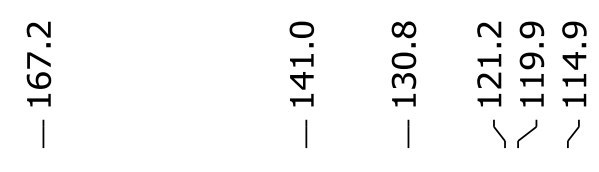

ตु่

$\hat{m}$

$\stackrel{-}{+}$

\section{$\mathrm{CDCl}_{3}, 151 \mathrm{MHz}$}

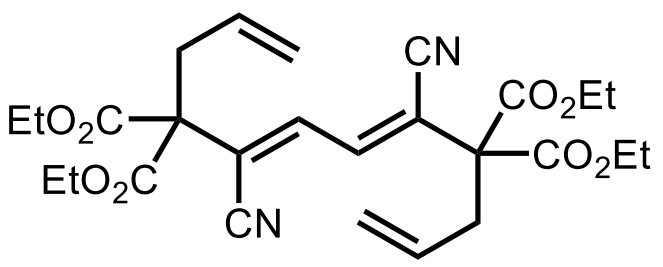

22

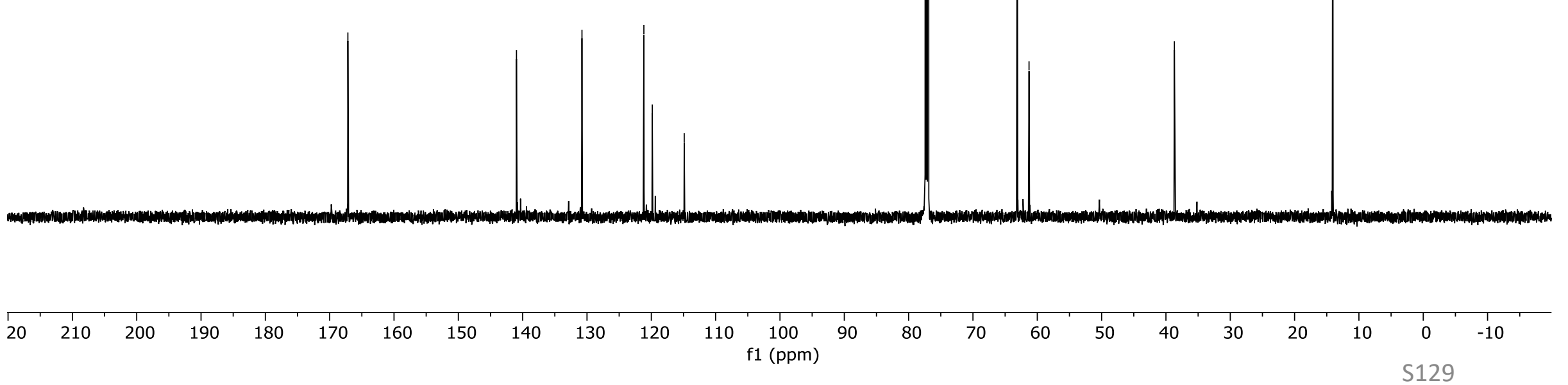




\section{$\mathrm{CDCl}_{3}, 600 \mathrm{MHz}$}
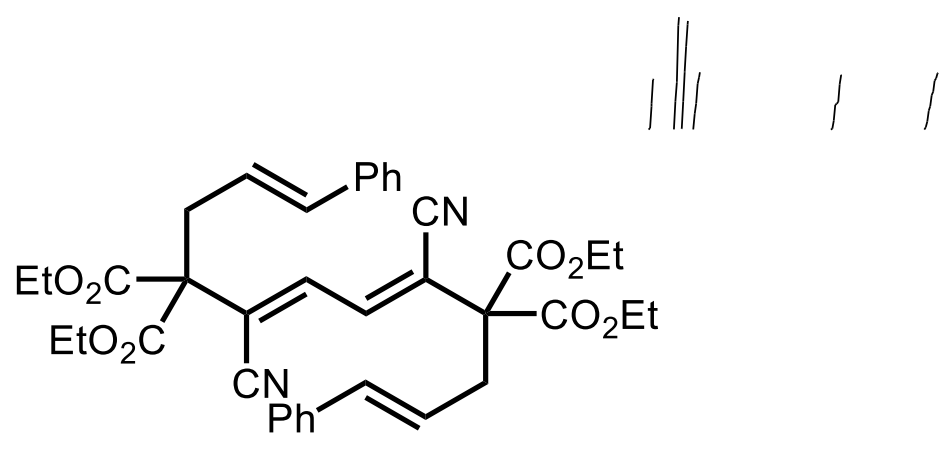

23

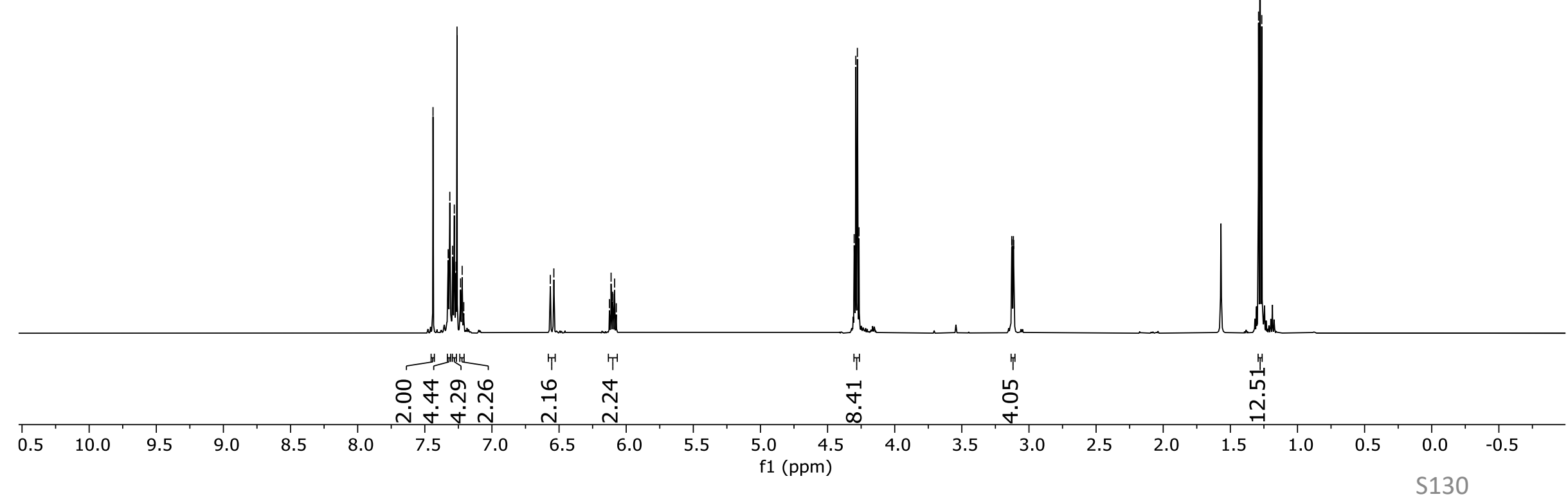




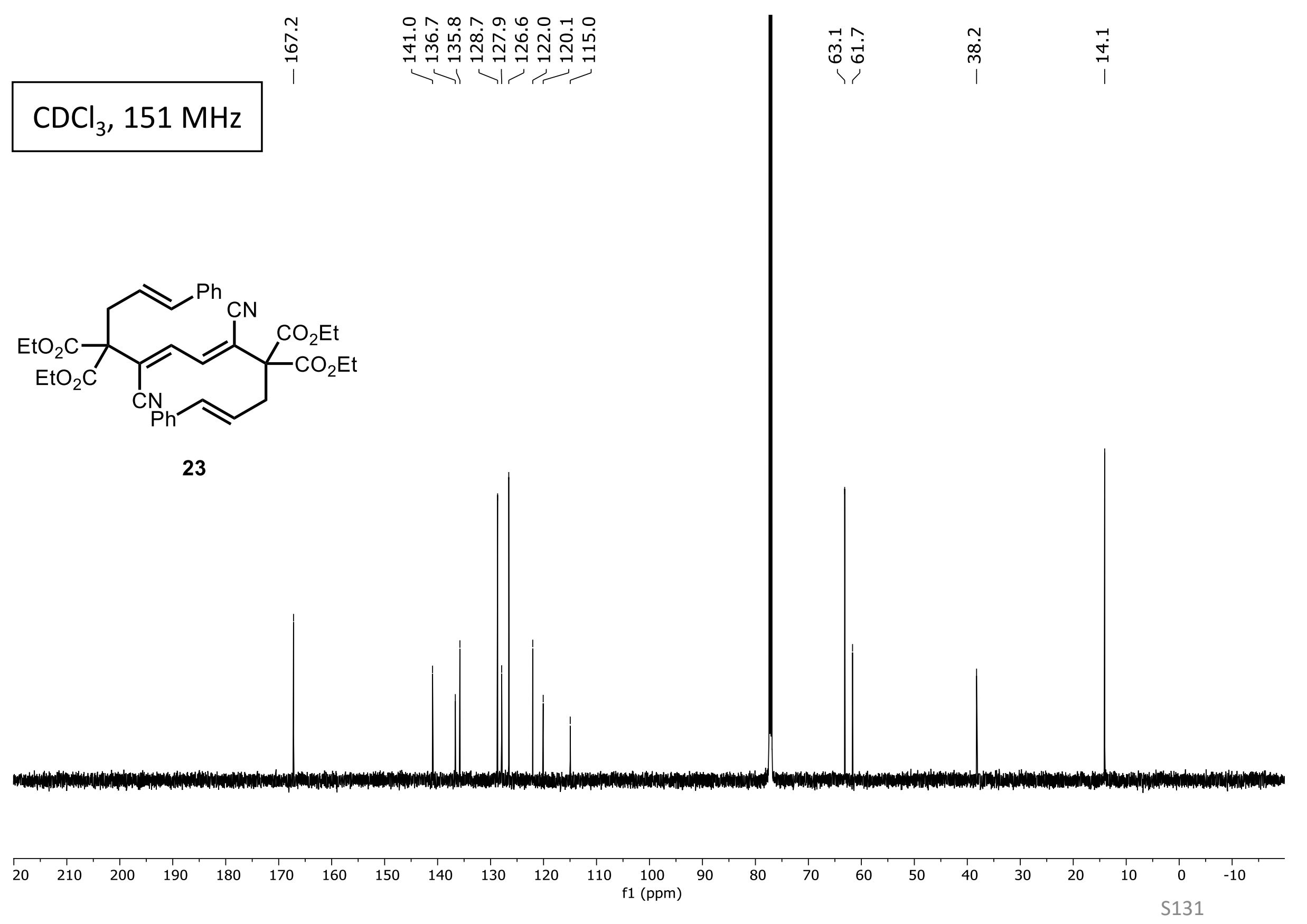




\section{$\mathrm{CDCl}_{3}, 600 \mathrm{MHz}$}

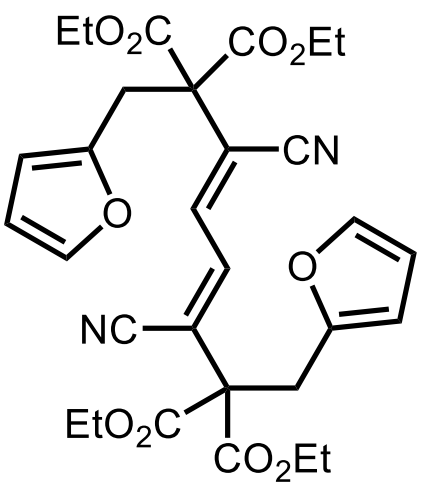

24

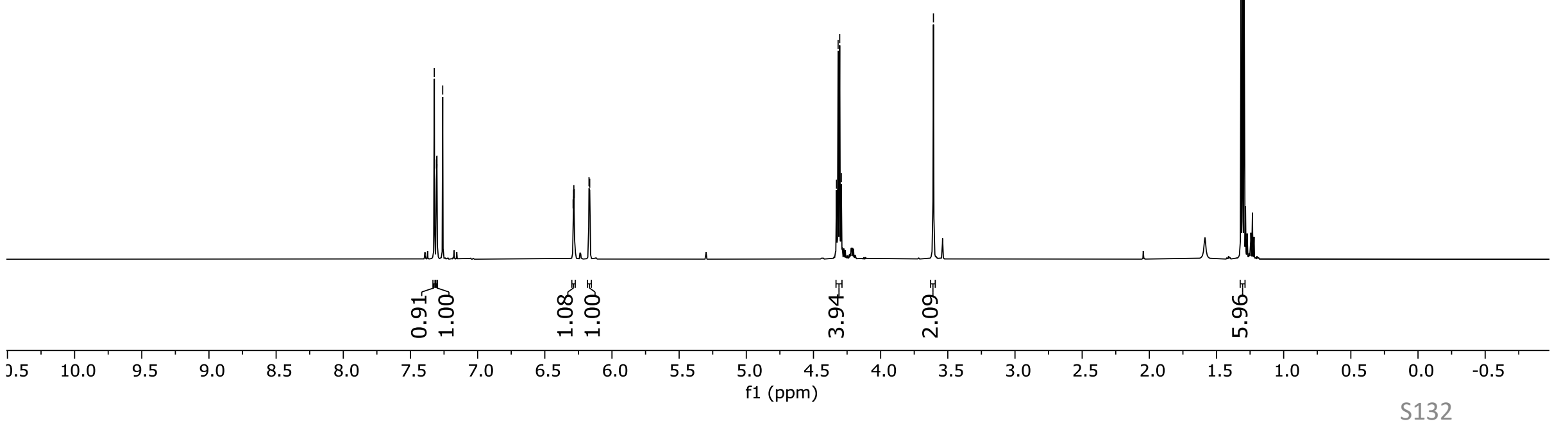



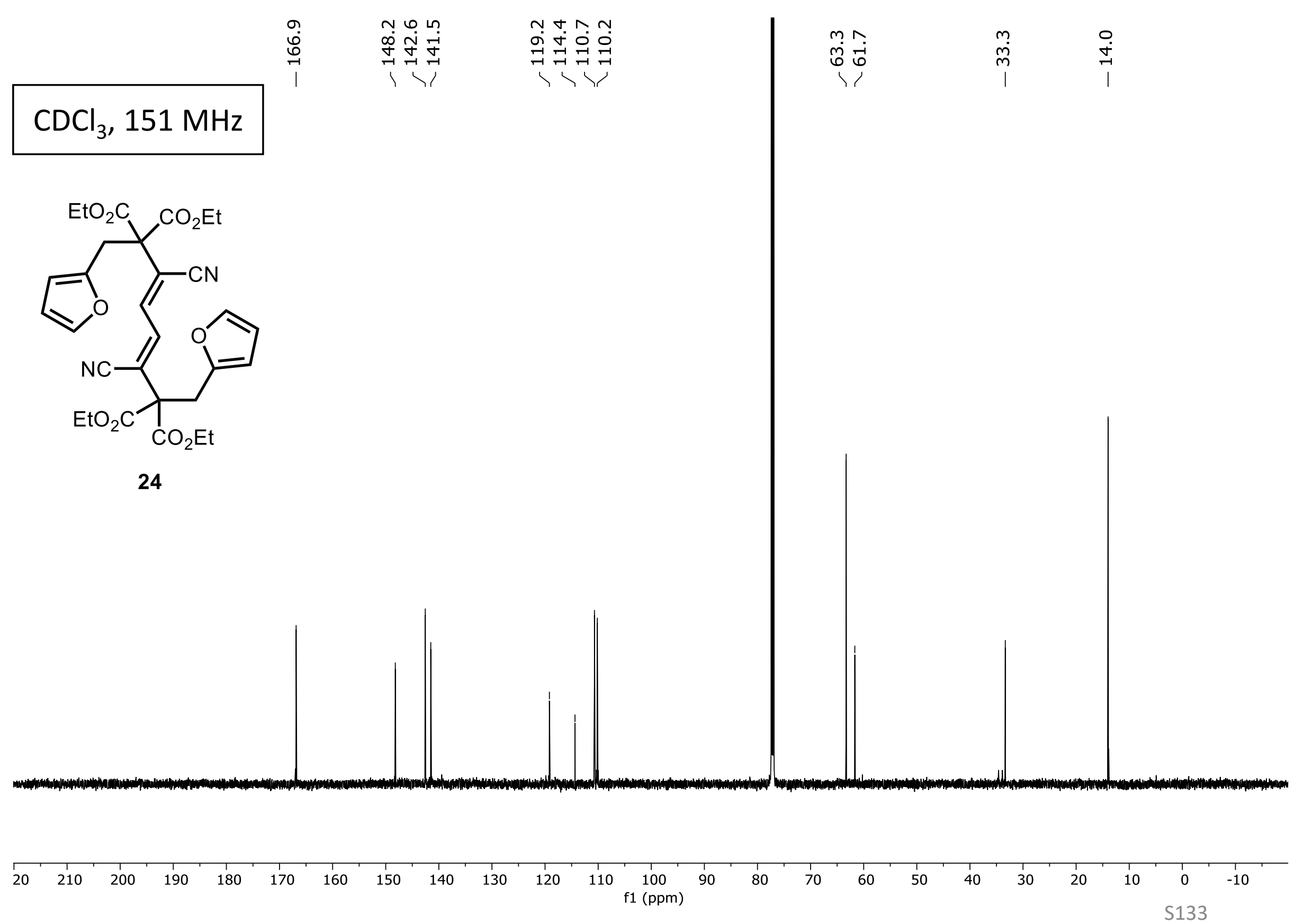


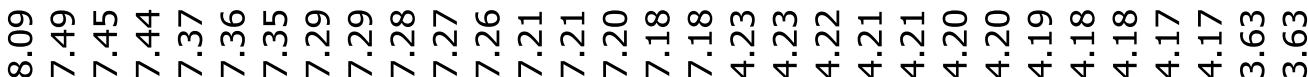

\section{$\mathrm{CDCl}_{3}, 600 \mathrm{MHz}$}

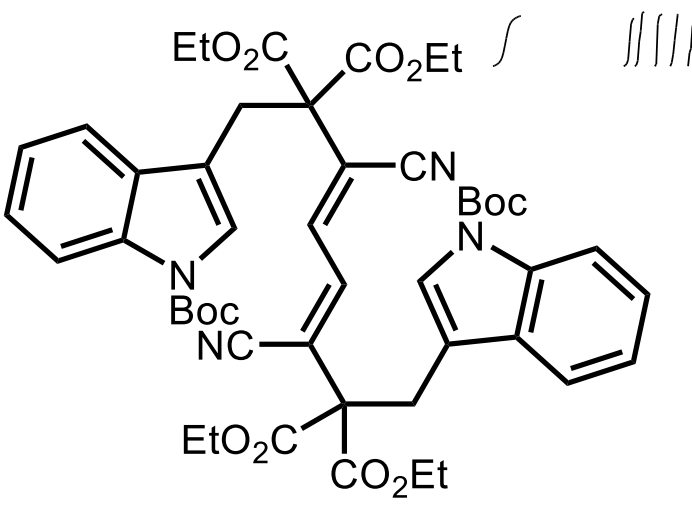

25

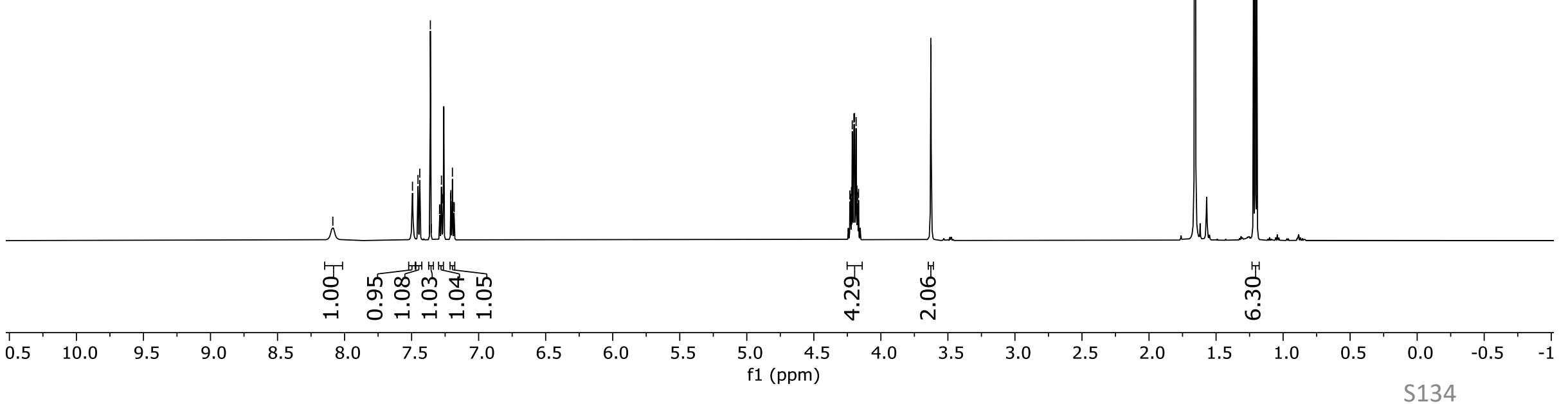



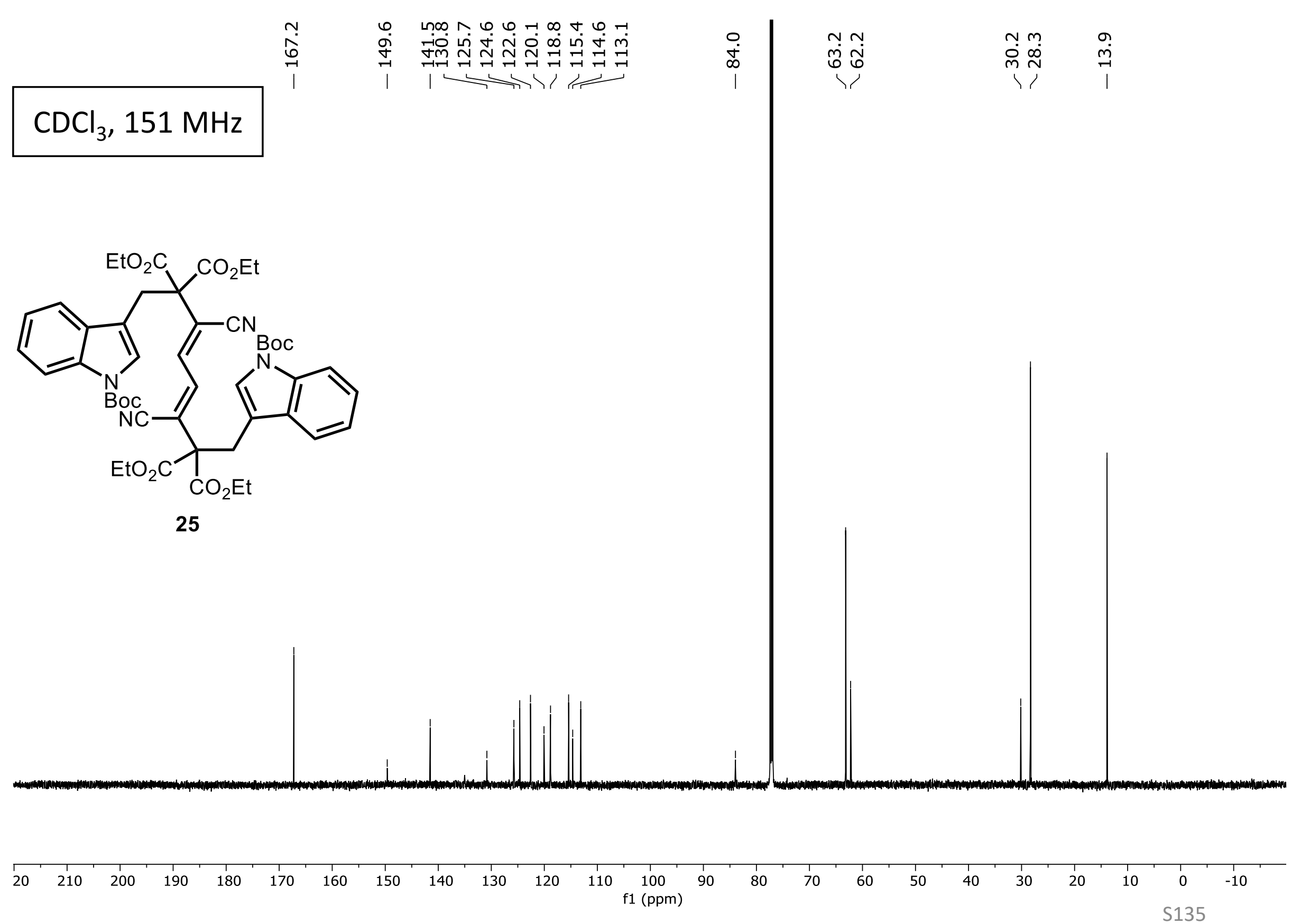


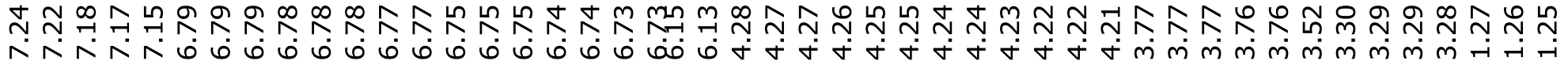

\section{$\mathrm{CDCl}_{3}, 600 \mathrm{MHz}$}
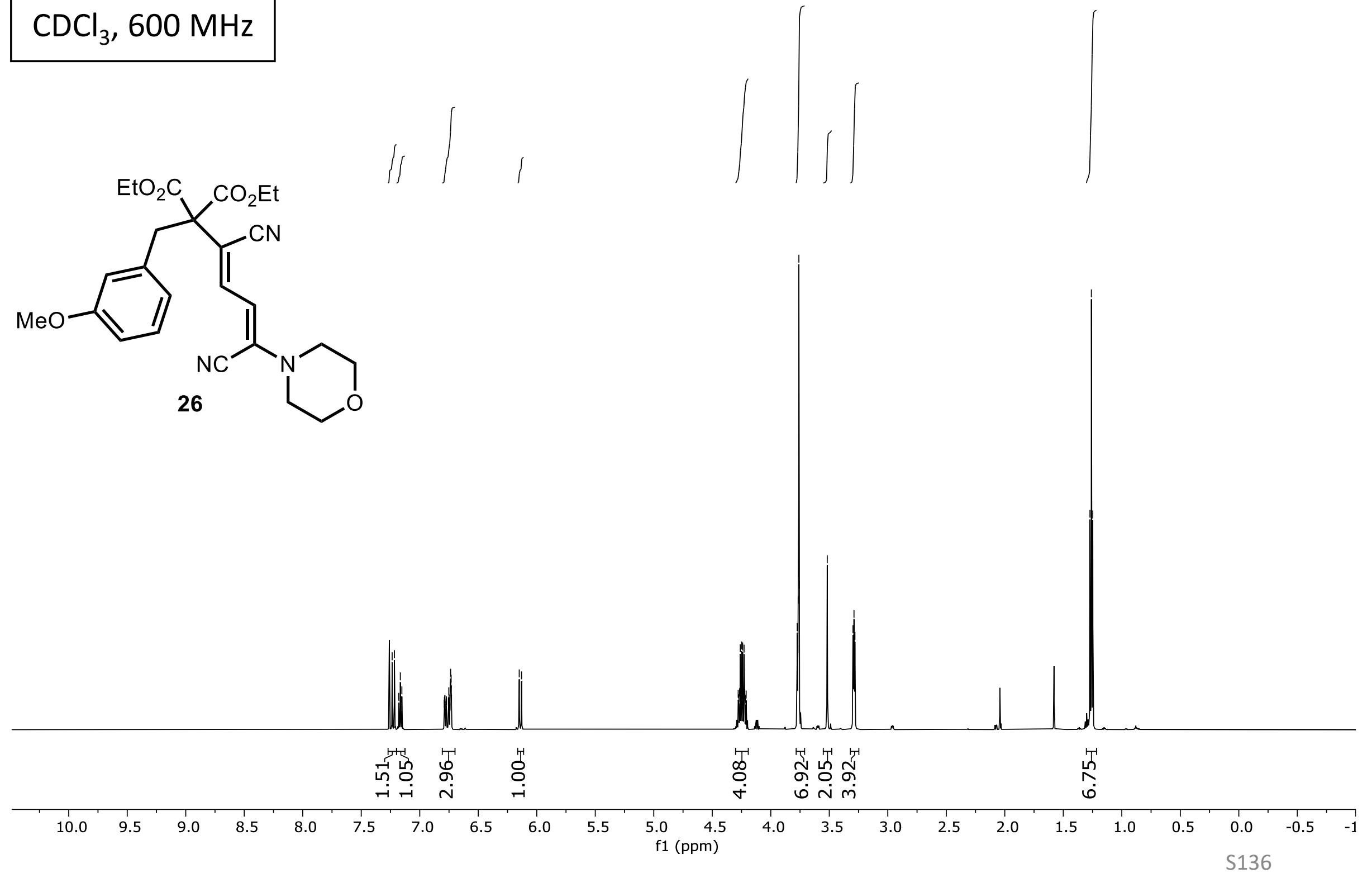


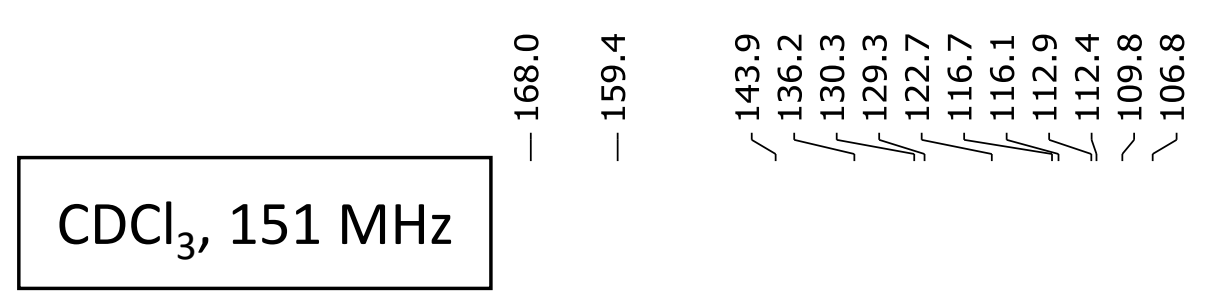

\section{$\mathrm{CDCl}_{3}, 151 \mathrm{MHz}$}
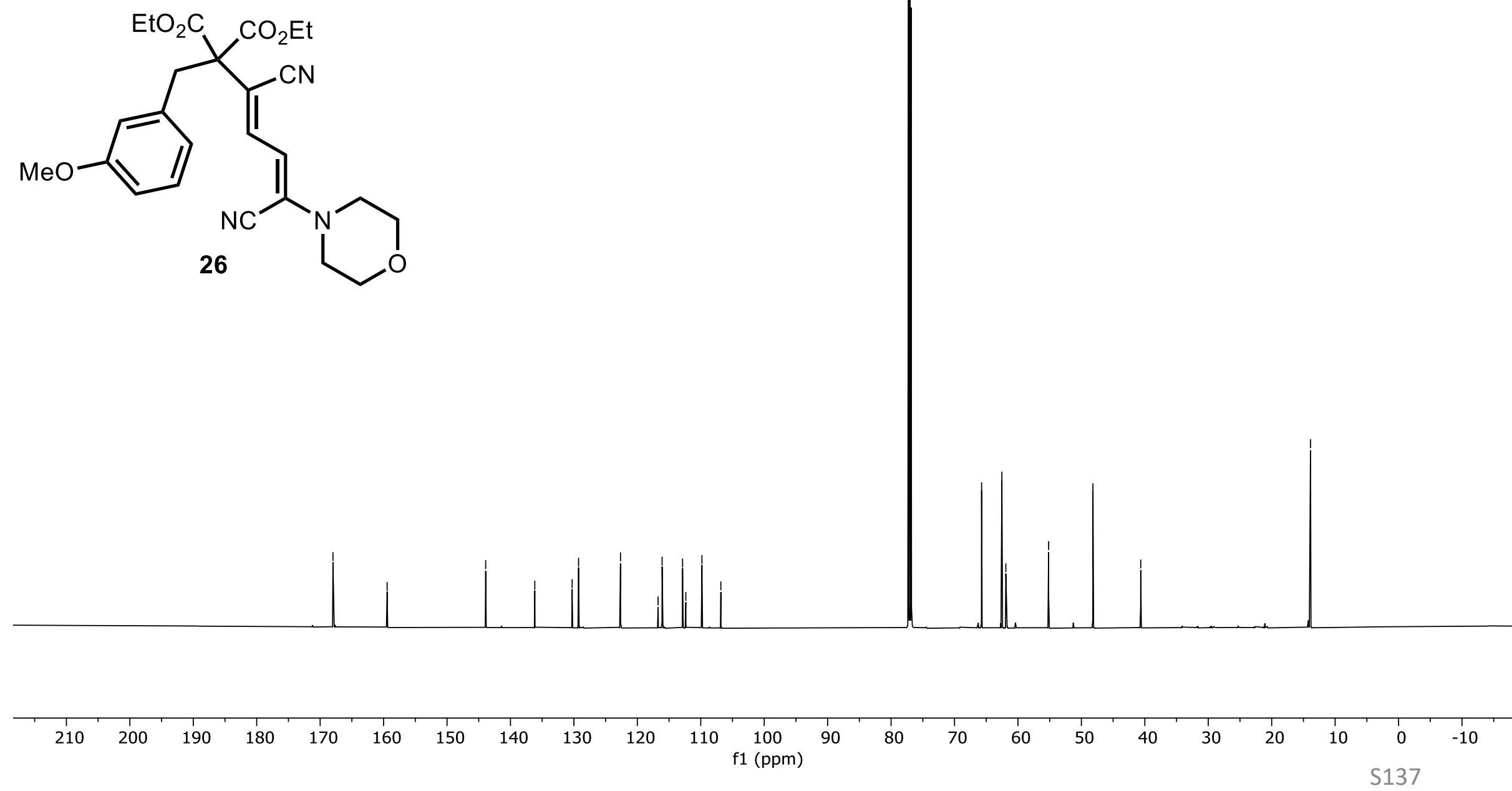


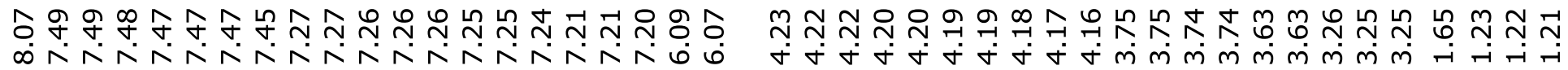

\section{$\mathrm{CDCl}_{3}, 600 \mathrm{MHz}$}
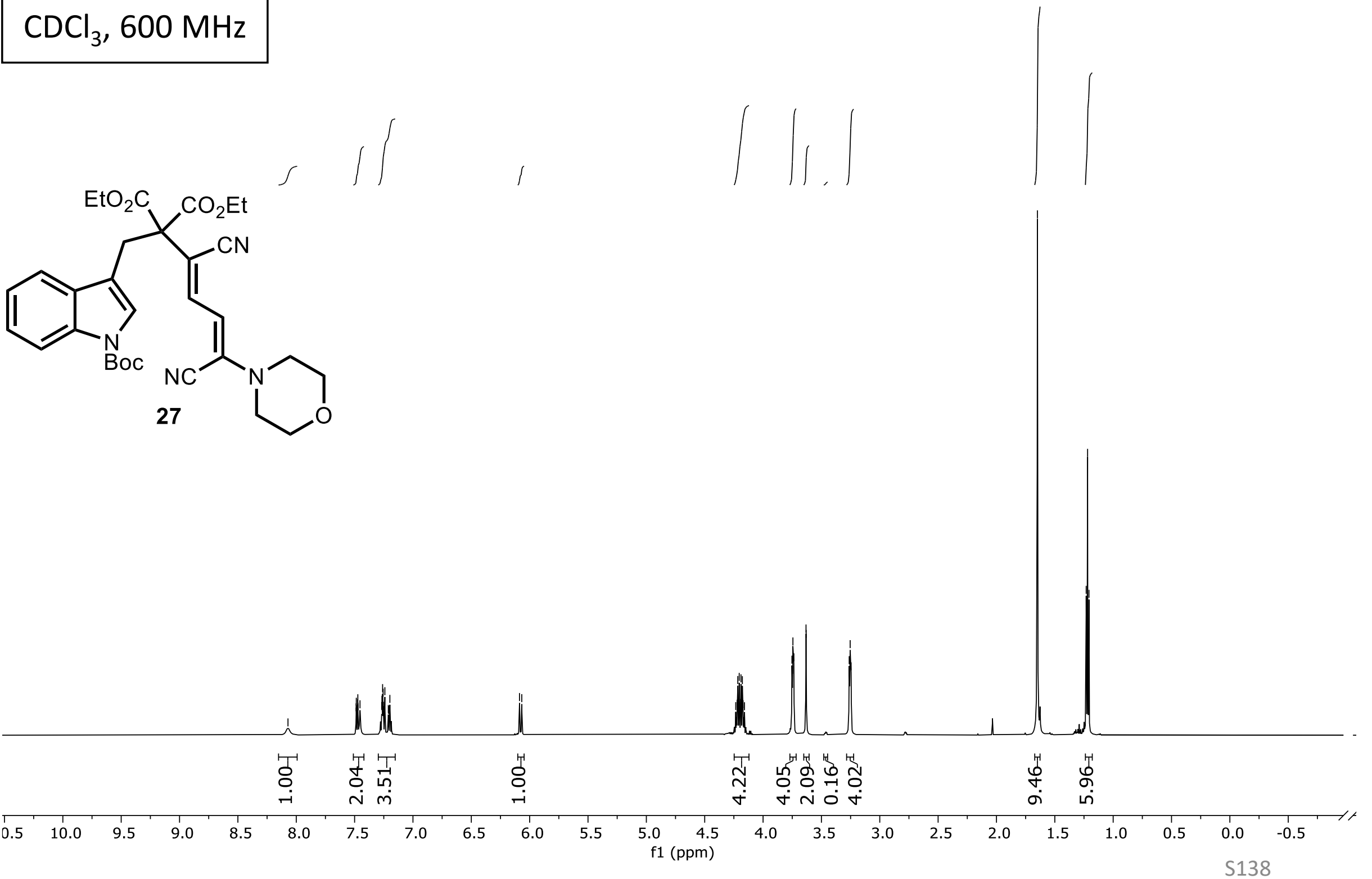


\section{$\mathrm{CDCl}_{3}, 151 \mathrm{MHz}$}
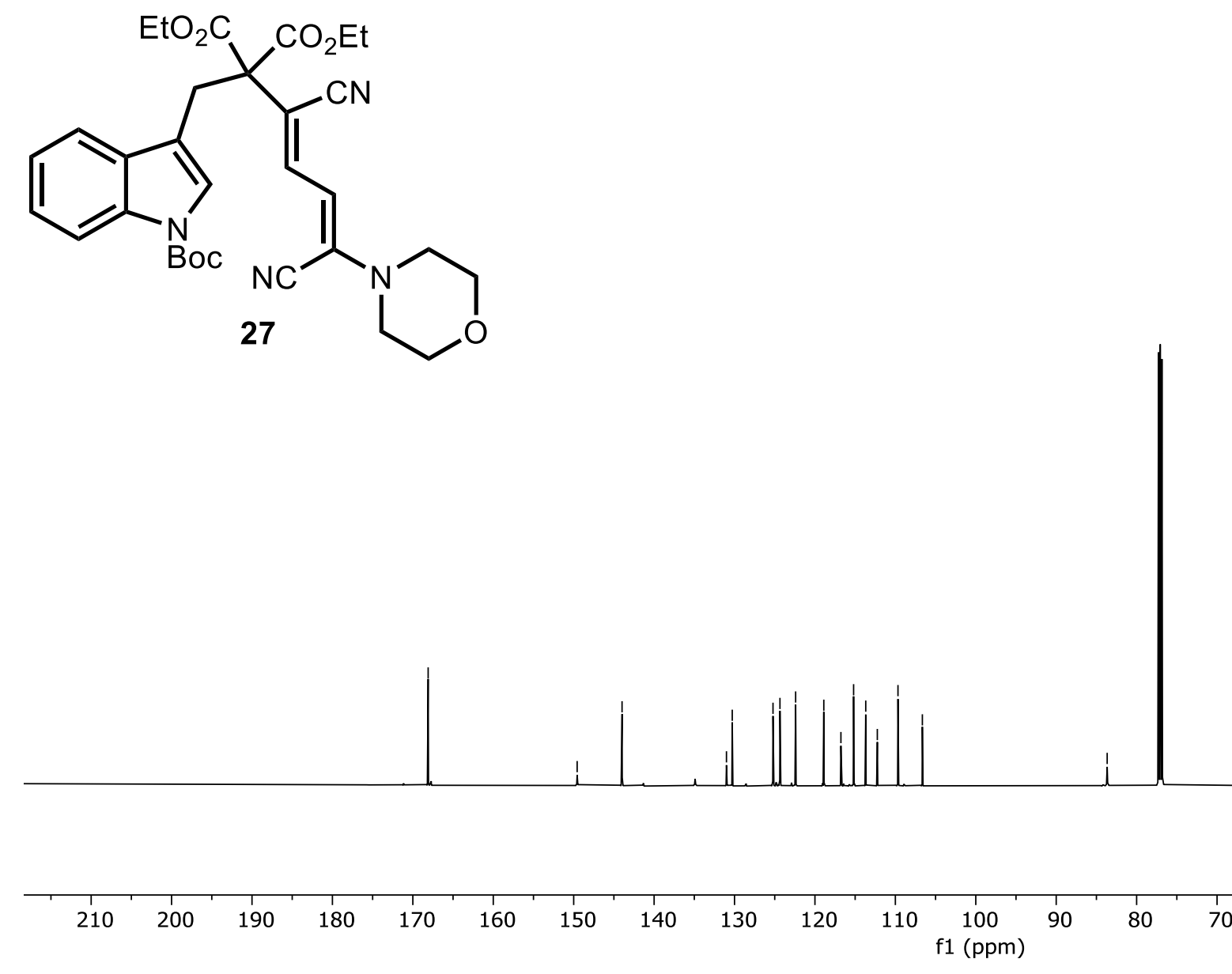

60
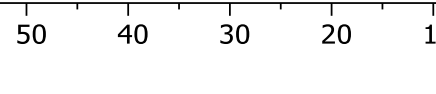


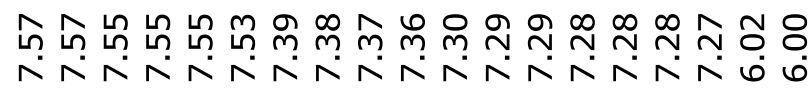

\section{$\mathrm{CDCl}_{3}, 600 \mathrm{MHz}$}
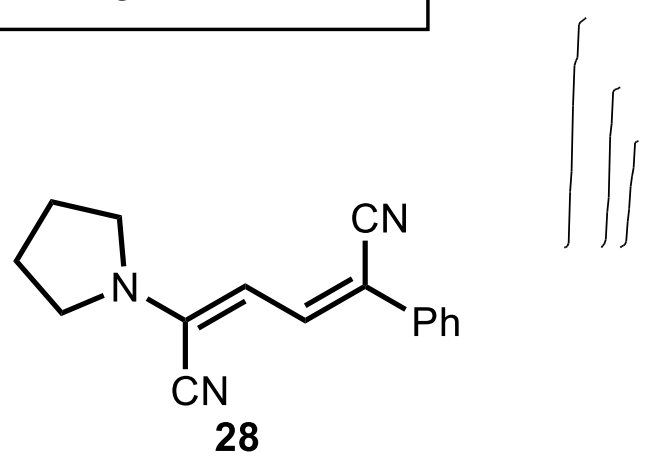

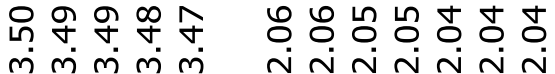
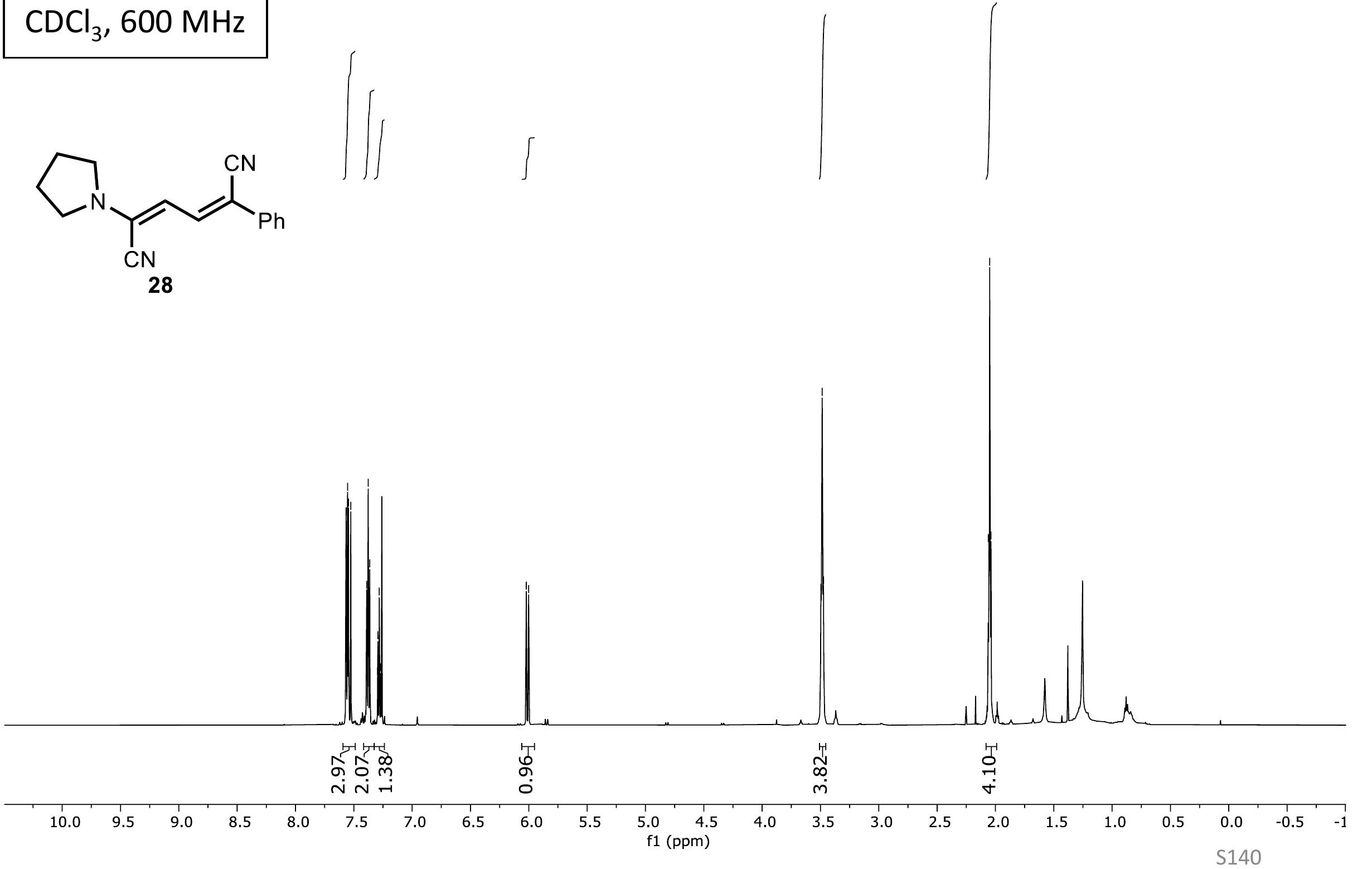


\section{$\mathrm{CDCl}_{3}, 151 \mathrm{MHz}$}

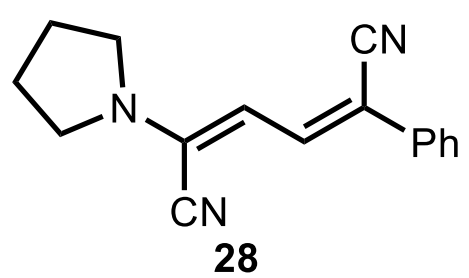




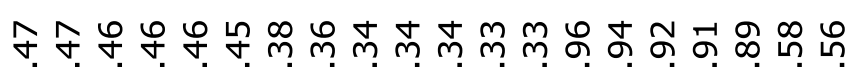

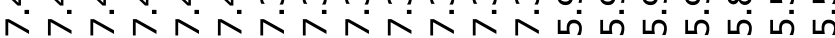
in in in in in

\section{$\mathrm{CDCl}_{3}, 600 \mathrm{MHz}$}
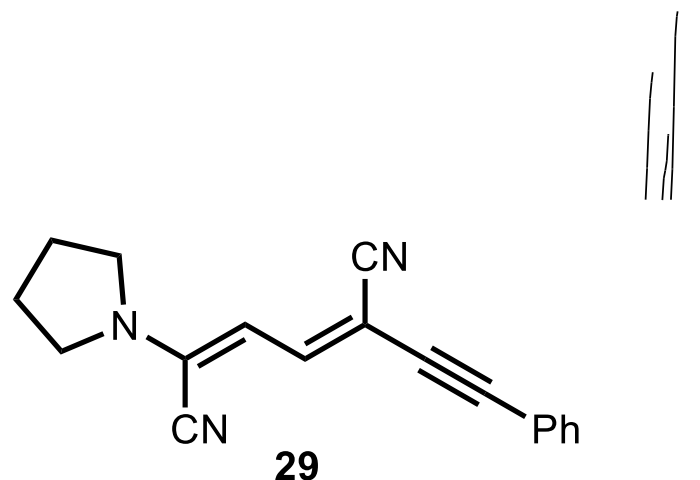

舟 $m m m m m$

$\longrightarrow$

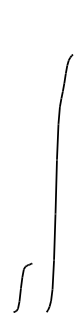

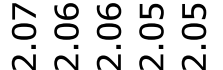

nin

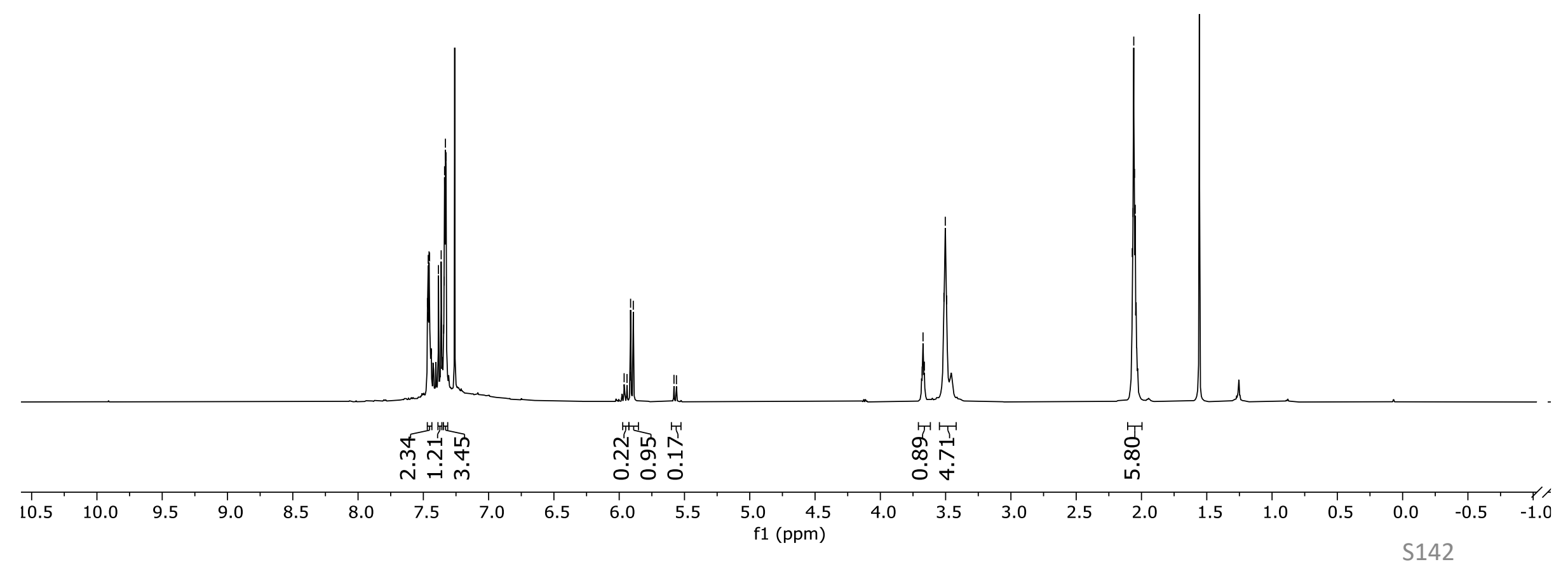




\section{$\mathrm{CDCl}_{3}, 151 \mathrm{MHz}$}
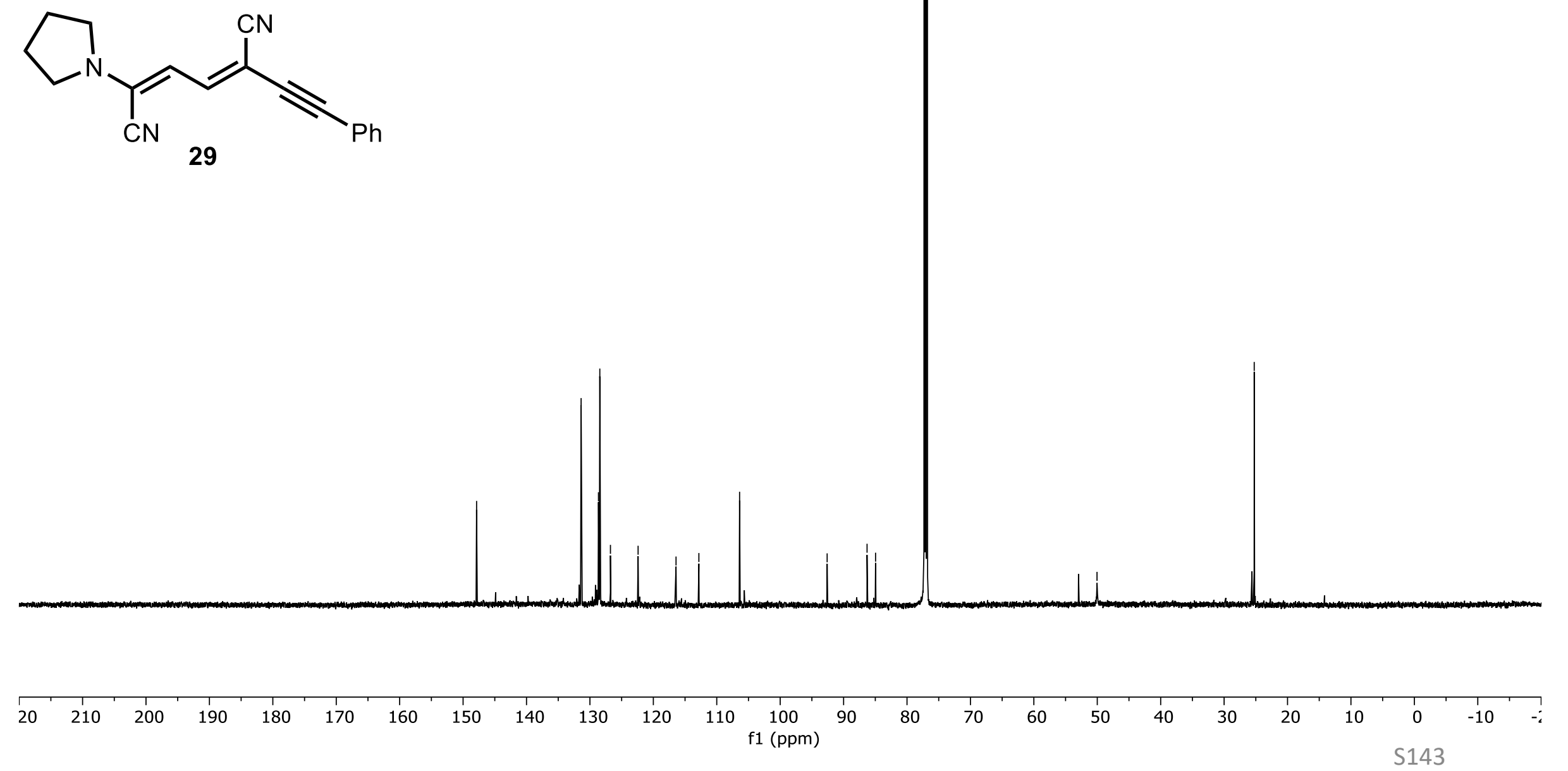


\section{$\mathrm{CDCl}_{3}, 600 \mathrm{MHz}$}
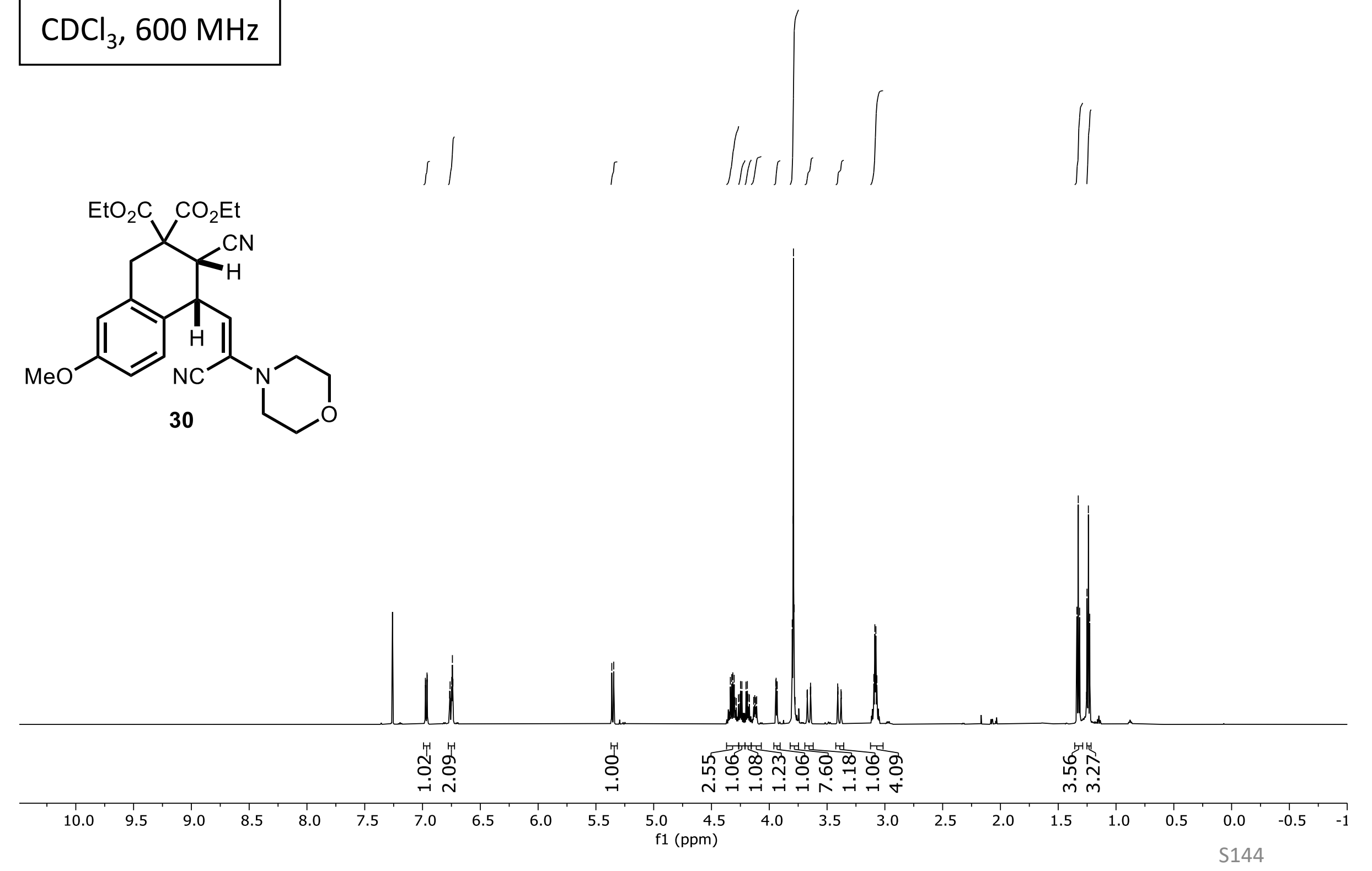

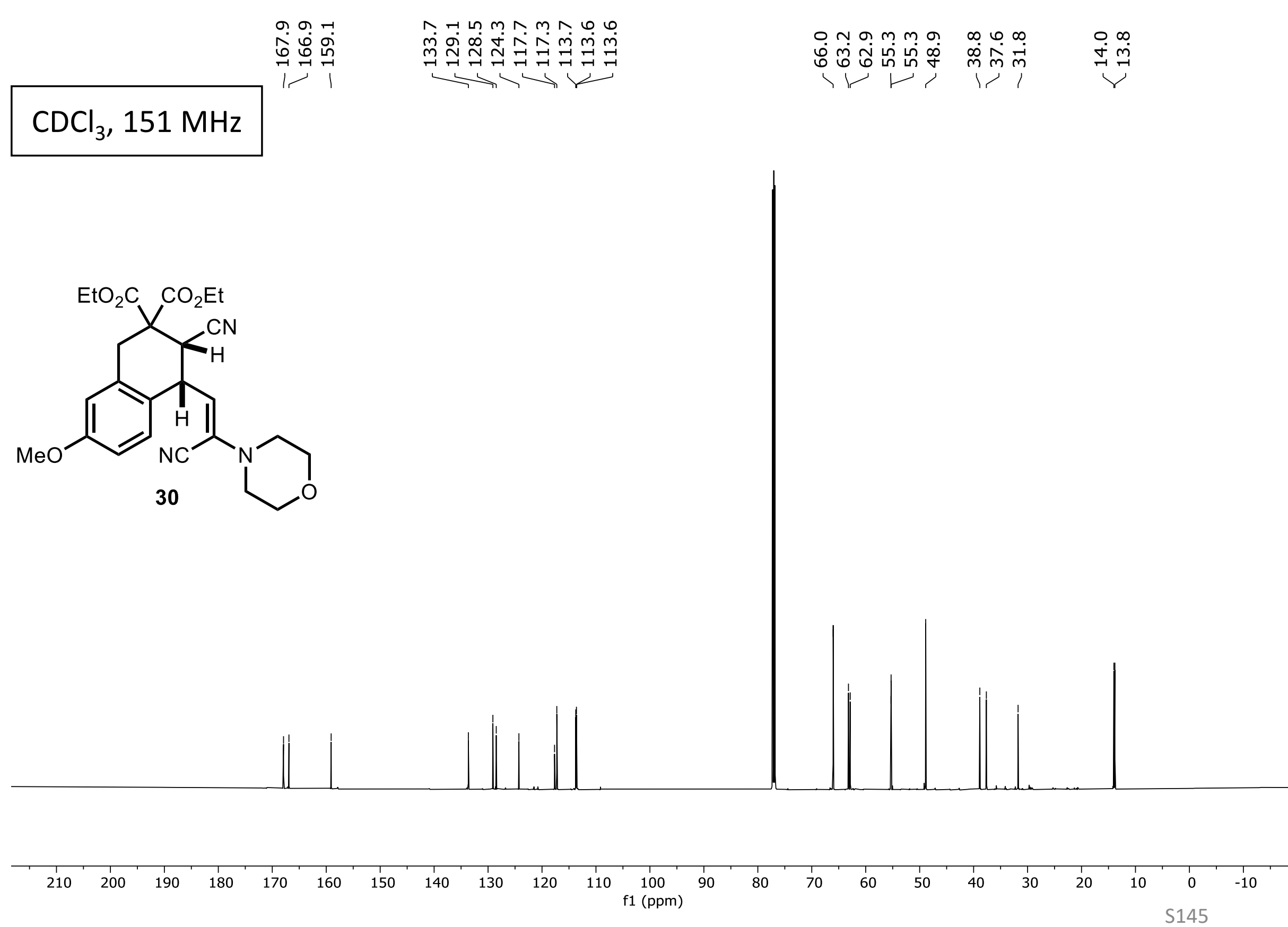

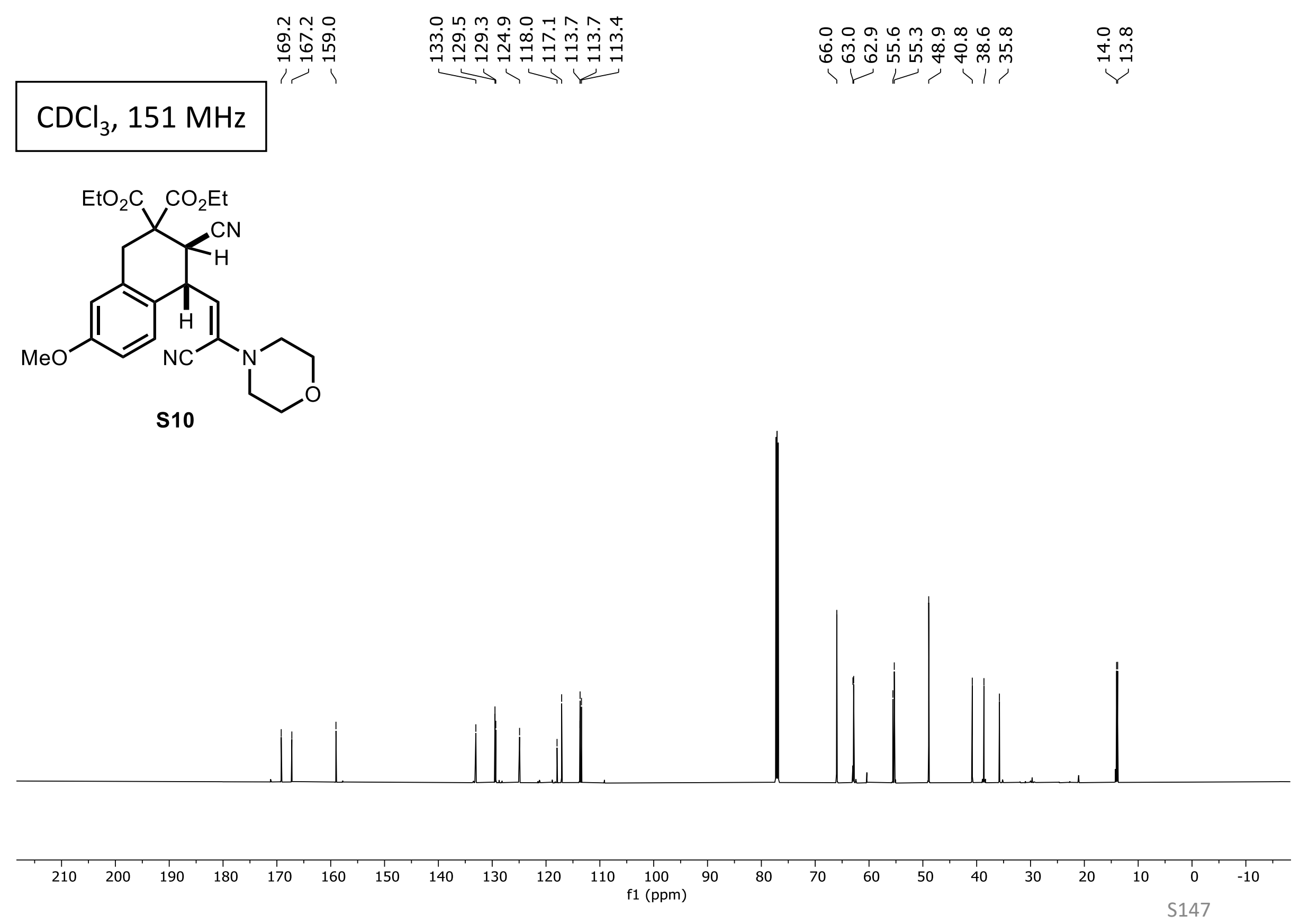


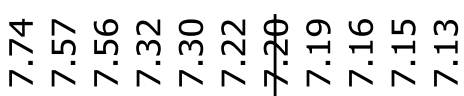

\section{$\mathrm{CDCl}_{3}, 600 \mathrm{MHz}$}

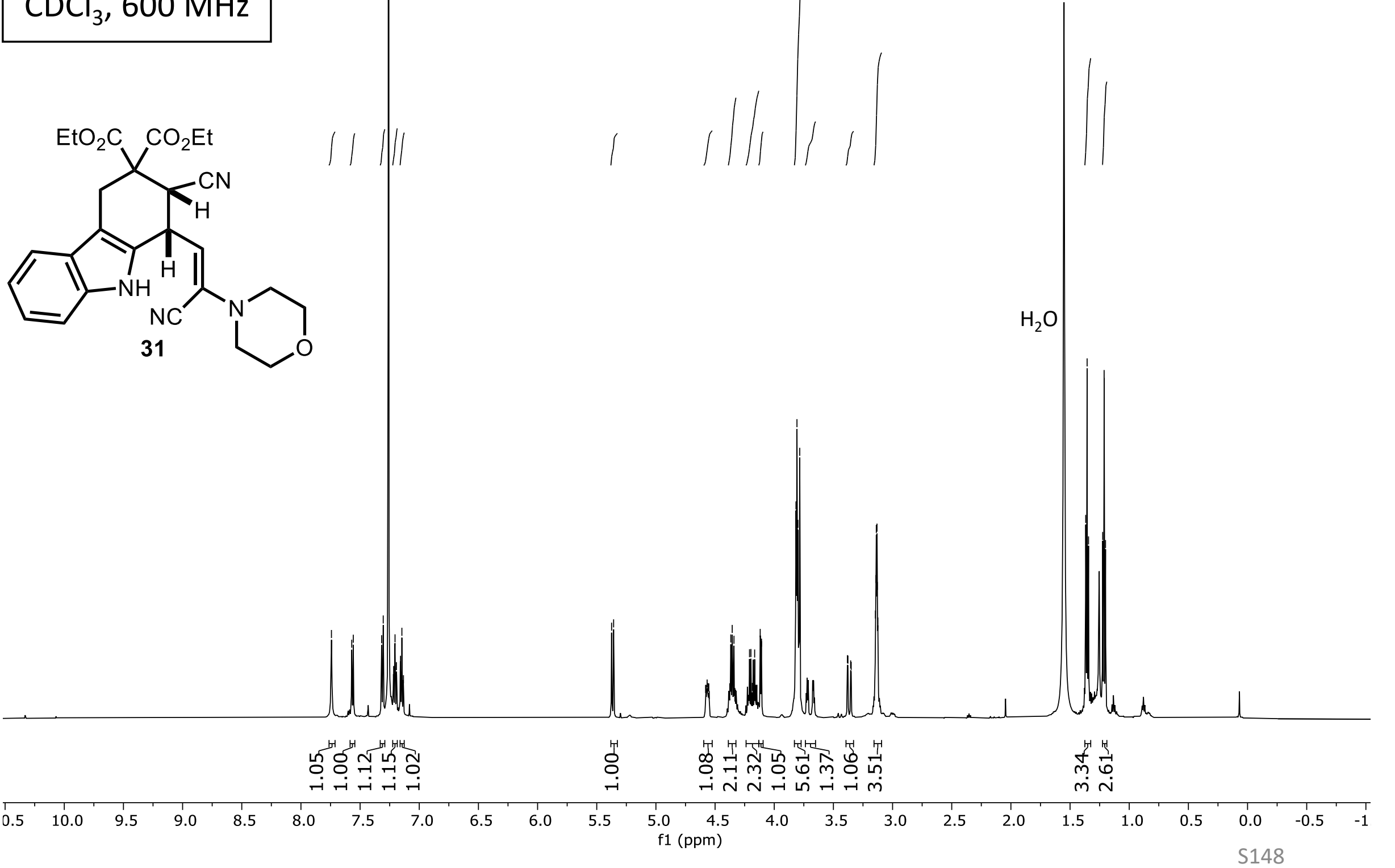

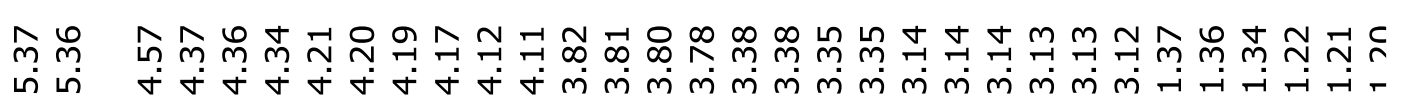



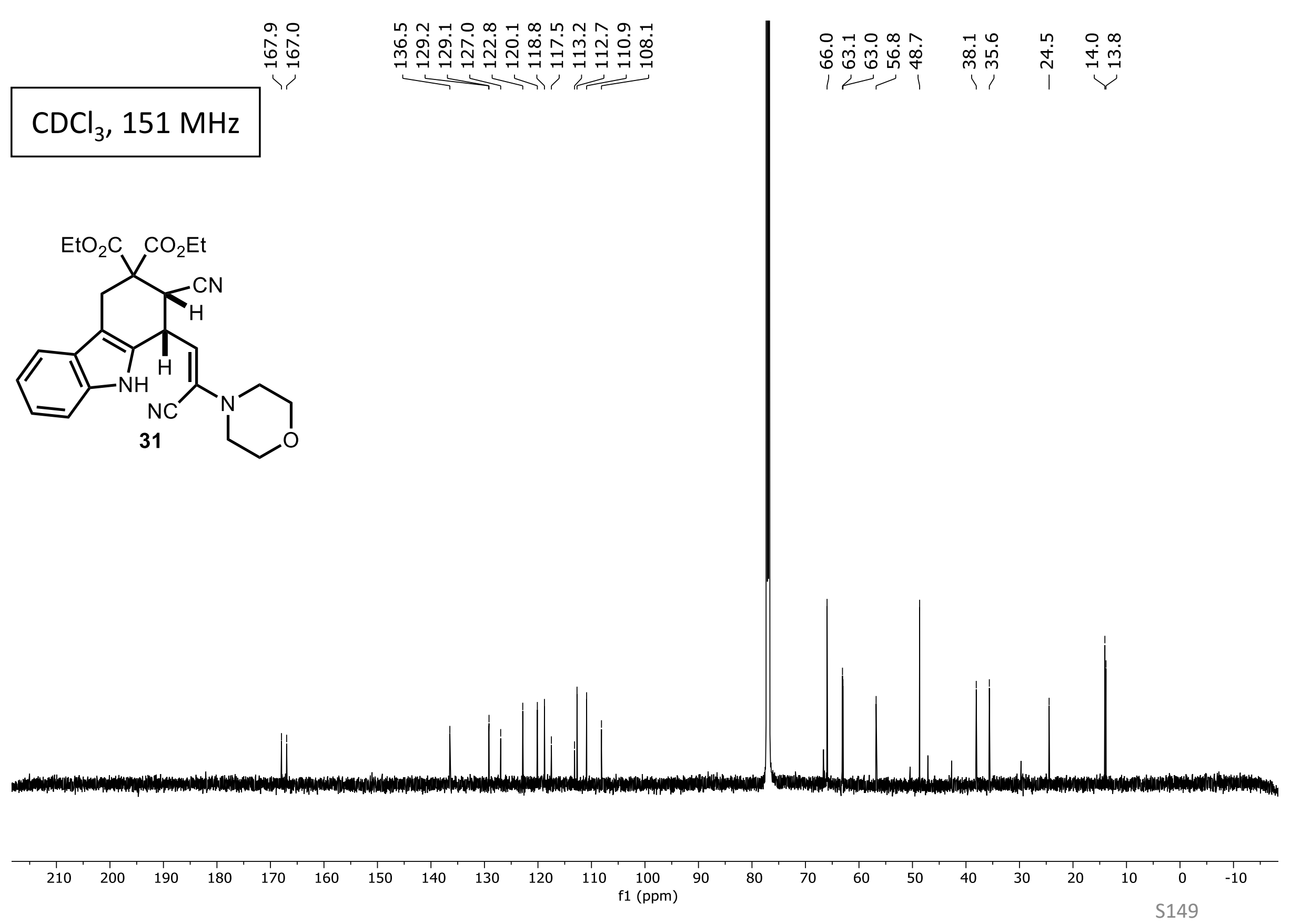


\section{$\mathrm{CDCl}_{3}, 600 \mathrm{MHz}$}
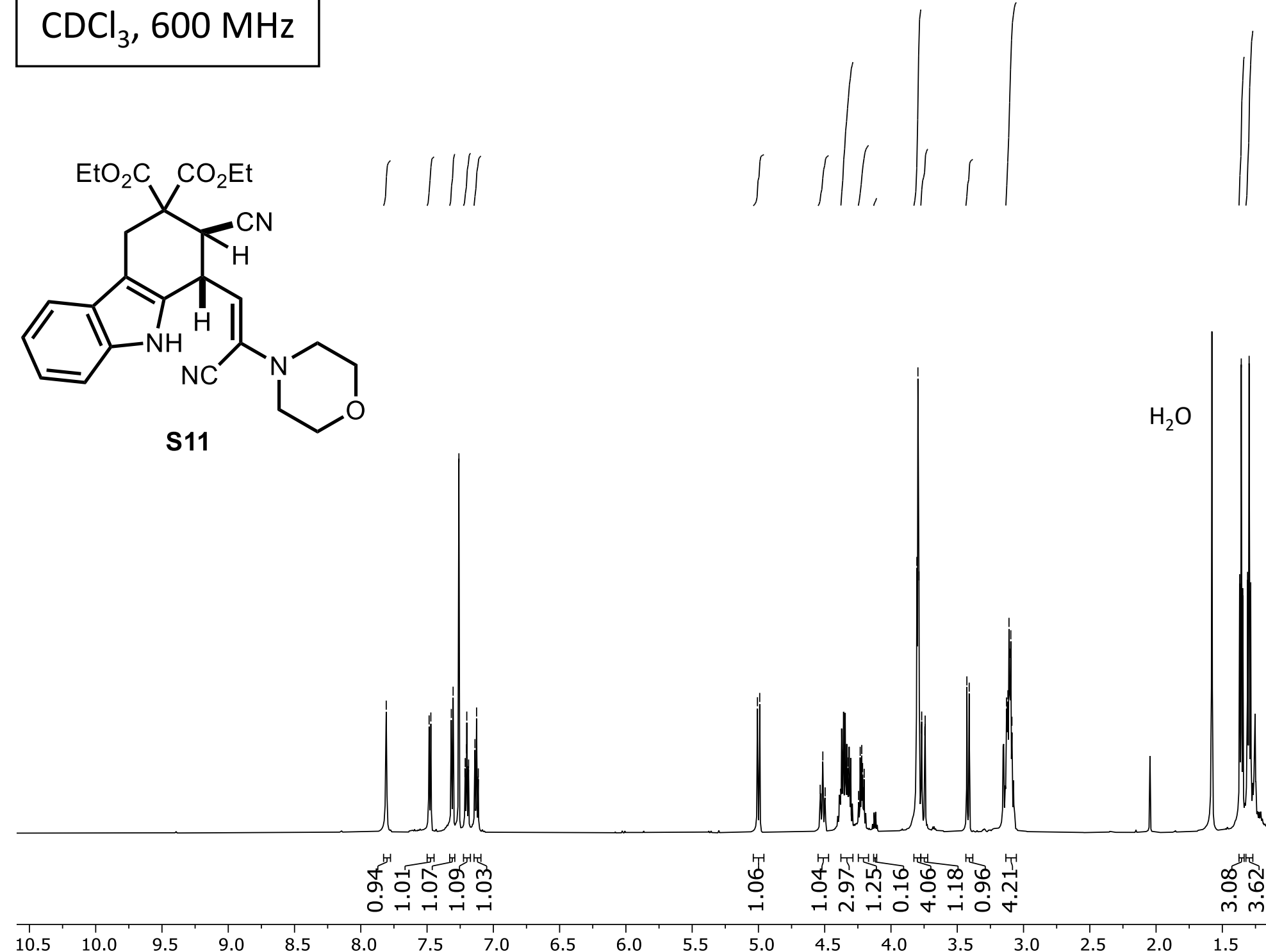
m 


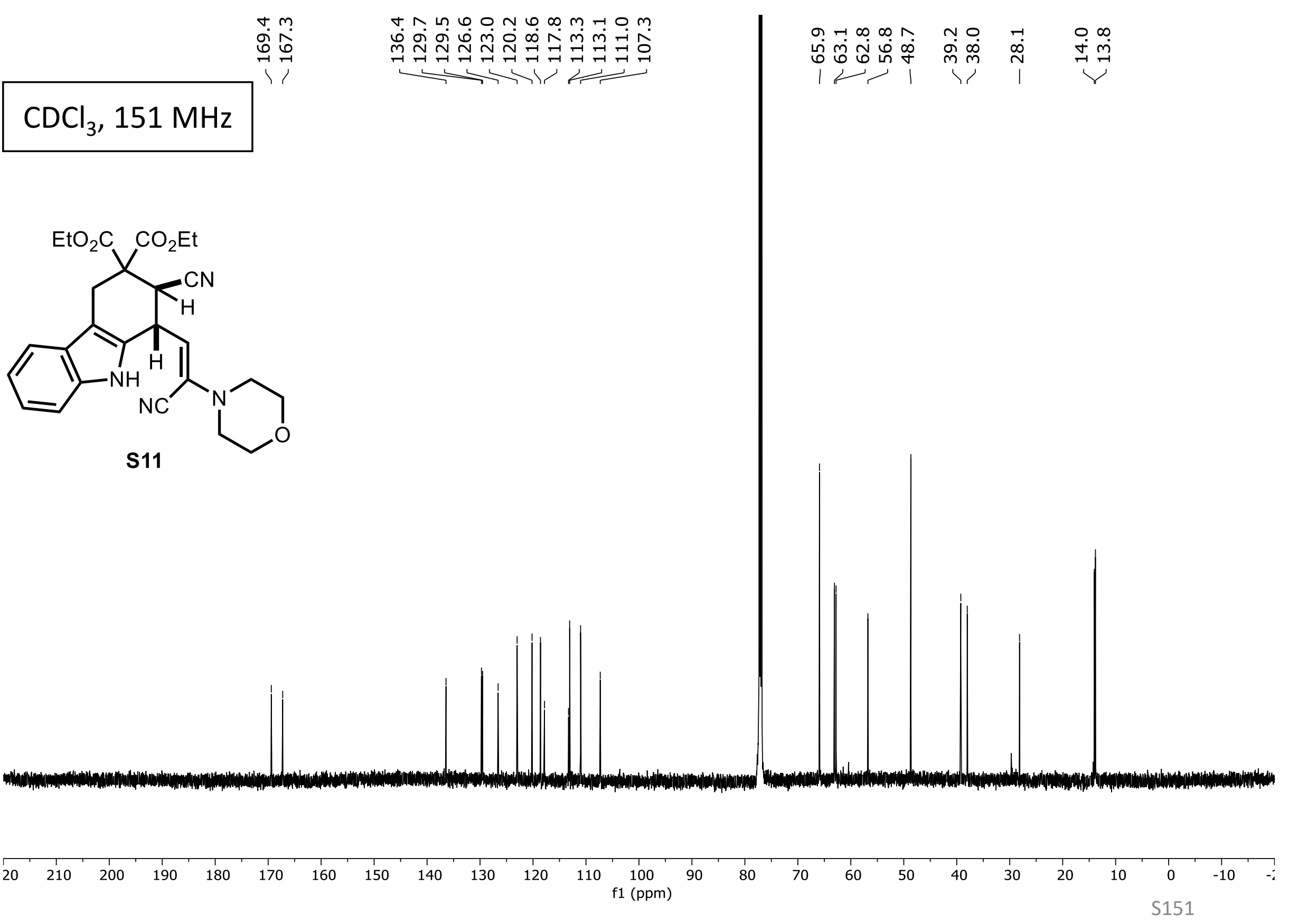

